$8.1596 \quad 5032874$

ECO-HYDRAULIC MODELLING OF

EUTROPHICATION FOR RESERVOIR MANAGEMENT 


\title{
Eco-hydraulic Modelling of Eutrophication for Reservoir Management
}

\author{
DISSERTATION
}

Submitted in fulfilment of the requirements of the Board for Doctorates of Delft University of Technology and of

the Academic Board of the UNESCO-IHE Institute for Water Education for the Degree of DOCTOR

to be defended in public

on Monday, December 7, 2009 at 10:00 hours

in Delft, the Netherlands
Prometheusplein
2628 ZC Deili

by

Nahm-Chung Jung

born in Kyungbook Province, Republic of Korea

Master of Science in Sanitary Engineering

UNESCO-IHE, the Netherlands 
This dissertation has been approved by the supervisor(s)

Prof. dr. R. K. Price

Members of Awarding Committee;

Chairman

Prof. dr. ir. A. E. Mynett

Prof. dr. R. K. Price

Prof. dr. Ir. H. H. G. Savenije

Prof. dr. ir. G. S. Stelling

Prof. dr. ir. D. P. Solomatine

Prof. dr. ir. J. P. O'Kane

Prof. dr. S. J. Hwang

Prof. dr. ir. N. C. van de Giesen
Rector Magnificus, TU Delft, The Netherlands Vice-chairman, UNESCO-IHE, The Netherlands

UNESCO-IHE The Netherlands, Supervisor

TU Delft/ UNESCO-IHE, The Netherlands

TU Delft, The Netherlands

UNESCO-IHE/ TU Delft, The Netherlands

University College Cork, Ireland

Konkuk University, South Korea

TU Delft, The Netherlands, reserve

CRC Press/Balkema is an imprint of the Taylor \& Francis Group, an informa business (C) 2009, Nahm-Chung Jung

All rights reserved. No part of this publication or the information contained herein may be reproduced, stored in a retrieval system, or transmitted in any form or by any means, electronic, mechanical, by photocopying, recording or otherwise, without written prior permission from the publisher.

Although care is taken to ensure the integrity and quality of this publication and the information therein, no responsibility is assumed by the publishers nor the author for any damage to property or persons as a result of operation or use of this publication and/or the information contained herein.

Published by:

CRC Press/Balkema

PO Box 447, 2300 AK Leiden, The Netherlands

e-mail: Pub.NL@taylorandfrancis.com

www.crcpress.com - www.taylorandfrancis.co.uk - www.balkema.nl

ISBN 978-0-415-57382-5 (Taylor \& Francis Group) 


\section{Summary}

\section{ECO-HYDRAULIC MODELLING OF EUTROPHICATION FOR RESERVOIR MANAGEMENT}

A reservoir is an artificial lake created when a dam or impoundment is constructed across a river. Reservoirs are designed for a number of purposes including water supply, flood control, hydropower generation, recreation, etc. Dams are often built to serve regions where there is considerable social, industrial and agricultural activity. Therefore they are exposed to pollution, which interferes with vital and legitimate water uses. This calls for intensive studies of the water quality of these reservoirs prior to or during their operation.

Lakes and reservoirs are considered as water bodies with similar physical properties despite certain differences, especially in operation and facilities. In particular, both bodies normally have a long retention time. A water quality study on a reservoir or lake and, indeed any other water body, involves characterizing three major components: hydrodynamics, physical-chemistry and biology. Traditionally, these characteristics are developed through an intensive monitoring programme involving the various environmental variables.

In most water quality monitoring programmes, detailed hydrodynamic measurements that describe water motion are rarely included because of budget and technical limitations. The emphasis is mainly upon general physical-chemical parameters such as water temperature, dissolved oxygen, $\mathrm{pH}$ and Secchi depth due to their ease of measurement. However, the prediction of the hydrodynamics (water motion and transport) of materials impacting on the water quality has long been the recognized as a crucial component of any water quality study. Generally, in a temperate monsoon area, reservoirs can undergo strong thermal stratification twice a year. This thermal stratification leads to density flows containing dissolved and suspended solids in the reservoir where there are differences in temperature between the receiving and inflowing water. Therefore, many recent research projects on reservoir water quality have focused on two kinds of eco-hydraulic modelling: physically-based deduction models using boundary data and data-based induction models using domain data.

With the increase of stress upon reservoirs due to the growing human/urban populations and their production activities, nutrient enrichment of the water body and its resultant increase in phytoplankton growth (a process termed eutrophication) can have direct effects on the water quality within the water body. Health hazardous and toxic substances, such as arsenic and heavy metal stored in a reservoir, also make the water body unsuitable for its purpose. This study focuses on eutrophication due to nutrient loads in a reservoir. The target water quality variable is algae, a kind of phytoplankton. Other factors, in addition to nutrient availability, can also enhance or limit the growth of phytoplankton, such as the amount of light available, water temperature, amount of 
phytoplankton being consumed by phytoplankton-eating organisms, toxic substances, residence time, hydraulic mixing, various watershed characteristics including morphology, soil type and land use etc. The eutrophication processes in the reservoir, being second order and/or third order activities, are affected by these many parameters. Since some of these parameters act against phytoplankton growth as inhibitors, the assessment of reservoir water quality should include various analyses including biochemical and hydraulic mixing processes. Thus this study focuses on the water quality assessment through bioassay, hybrid modelling combining physically-based numerical modelling and data-driven modelling, and finally application for supporting decision making support for eutrophication management in the Yongdam reservoir.

\section{1) Assessment of water quality}

A reservoir water quality assessment focuses on physical-chemical parameters, eutrophication processes and organics. In spite of the short term period of record (20052007) for the Yongdam reservoir, the data reveals different flow regimes e.g. a double thermocline and turbid density current in 2005 and 2006 and a single thermocline and no turbidity current in 2007 due to meteorological conditions such as rainfall patterns. The weather condition influences not only the mixing of the reservoir water such as affecting the thickness of thermocline that determines the path of the nutrient transport and advection in the reservoir but also the algal growth. Nevertheless, decreasing patterns of Chl-a and phosphorus concentration over this study period (2005-2007), imply that the organics produced from the watershed and algal decay are increasing on the bottom of the reservoir. The increasing trend of COD in the entire reservoir and decreasing trend of $\mathrm{DO}$ in the bottom layer over the three years have identified that eutrophication processes of the young Yongdam reservoir ever since it commenced filling with water in 2001 have stabilized over the three years since 2002-2004. This is referred to as an aging process. According to the water quality analysis over the subsequent three years (2005-2007), the Yongdam reservoir is slowly going through limnological evolution as it responds to the impacts of watershed.

A bioassay is the most direct and effective way of determining the amounts of different nutrients available to organisms in the water body; it forms a kind of limiting nutrient and eutrophication potential of an aquatic system. The significance of a bioassay measuring algal growth potential (AGP) in a water sample is that a differentiation can be made between a visible algal concentration and additional algal growth (potential) due to the available nutrient remaining in the sample. A high AGP in the surface water may indicate that there are unknown factors against algal growth. The AGP Test (AGPT) shows that the Yongdam reservoir is a phosphorus limited water body and identifies reservoir zones (riverine, transition and lacustrine) due to vertical and horizontal distributions of AGP during the strong thermal stratification of the reservoir water.

\section{2) Modelling paradigm}

In most scientific research, recorded data is compared with a theory founded on an 
analytic expression of physical laws. The success, or otherwise, of the comparison is a test of the hypothesis of how nature works as expressed by the mathematical formula. Alternatively, fitting a mathematical model to the data might determine certain physical parameters. Physically-based models predict, simulate or generate a phenomenon based on an analytic expression of physical laws. Therefore physically-based models can be used for various objectives: analysis, operation, prediction and planning. So, modellers want to look at results of modelling on consistent grids, time resolution and coordinate systems, but decision makers want to allow a user to incorporate the results quickly and easily into decision making. This requires obtaining a theoretical generalisation automatically from the results by means of induction. On the other hand, there can be weaknesses such as uncertain theories and long computing times for a domain discretised by a fine grid in the physically-based 3D modelling context. This is the reason that one- or two-dimensional models are still used as tools for decision making. Together with the weaknesses of hydrodynamic models, a wide range of environmental problems resulted in data-driven modelling emerging as a promising new direction of research that is intended to make sense of multivariate data. Such data-driven modelling has been proved to be attractive in many application areas. Fundamentally, data-driven modelling is the process of exploring relationships among a large number of variables and quantities of data in order to extract meaningful information from the data, in the form of formulae, computer codes, patterns or rules. However, many data-driven models have opacity in knowledge inference processes and require a huge amount of data. Therefore data-driven models are used mainly for forecasting or prediction with historical data. In order to overcome the weaknesses in each model, a hybrid model, which is a combination of the two kinds of models, is regarded here a new paradigm for the better understanding of nature.

\section{3) Physically-based numerical modelling}

Purely hydrodynamic studies on water bodies are very rare especially for inland water bodies; what is more popular is a water quality study which is based on a hydrodynamic model. This study carries out a hydrodynamic analysis of the water flow in a reservoir using a fully three-dimensional (3D) hydraulic model and water quality analysis using a laterally-averaged two-dimensional (2D) model calibrated with physical-chemical measurements. Most 3D models used in physical limnology are designed mainly for the simulation of ocean and estuary circulation. Many of these models have been successfully applied to oceanic and estuarial problems but there have been very few reported lake/reservoir applications. The reason for this is that there are different physical processes in reservoirs than in oceans. In lakes and reservoirs, the advection in the momentum equations is caused by internal waves, thermal gradients, winds and river inflows, whereas the Coriolis forces and pressure gradients are the forcing functions. Therefore, in order for the ocean models to capture the physics of reservoirs they need a solution with small length scales of motion compared to an ocean. As an alternative, laterally averaged 2D models have been applied to environmental modelling in reservoirs and lakes. This is because they are designed specifically for reservoirs and lakes, and some researchers claim their success is attributed to the ability of these models to capture 
accurately the large scale physics of the reservoir (though they hardly depict the small scale motions). These models are much more efficient in their use of computational memory and time than the 3D models. In this study, MIKE3D and CE-QUAL-W2 models, which have been developed primarily for ocean and reservoir applications respectively, have shown reasonable results for the reservoir mixing behaviour. The laterally-averaged two-dimensional model, CE-QUAL-W2, can be successfully used for simulation of the global mixing behaviour in the Yongdam reservoir except for the eutrophication process. The three-dimensional MIKE3D model, using a 90 meters horizontal and 2 meters vertical grid, simulated the hydrodynamics in the reservoir well. The results from MIKE3D show better replication of the observed hydrodynamics than the 2D model and reveal that a spillway release of flood water impacts on flows over the whole depth despite thermal stratification. This means that an efficient selective withdrawal of turbid water should be less than the rate at which water is drawn from the whole depth. The main forcing functions of mixing in the Yongdam reservoir are identified as air temperature, and inflow and outflow, especially spillway release. Since the parameters related to biological processes, such as the growth of algae, are not effectively fitted with measured data, the Chl-a concentration in the reservoir is predicted by a data-driven model using the measured data and the data set generated from the CE-QUAL-W2.

\section{4) Data-driven modelling}

In this study three machine learning methods for data-driven modelling (DDM), including partition (tree and regression), instance based rule of $k$ number(s) and a clustering rule, are tested. ANNs is a computer intelligence based agent classifier (a kind of regression), $k \mathrm{NN}$ uses an instance-based rule, MTs use a rule of partition, and $k$-means is a clustering method. Clustering identifies instances that bear a stronger resemblance to each other than to the remaining instances. Most numerical regression methods use least squares error regression (LSER). In this study, partial least squares regression (PLSR), in which latent vectors are found that explain both the response and predictor variation, are tested an alternative to LSER because of the lack of data. Model trees (MTs) are known as one of the most explicit DDMs methods, because they are tree-structured regression models that associate leaves with multiple linear regression functions. The DDMs were trained with measured data for two years (2005-2006), and then predicted concentrations of Chl-a (response variable) are compared with new input predictor variables (water temperature, $\mathrm{PO}_{4}-\mathrm{P}$ and total phosphorus) measured in 2007. According to the results of the application of these DDMs, the combination of $k$-means clustering and PLSR showed better results than other machine learning algorithms.

\section{5) Hybrid modelling}

The physically-based modelling has also a short-coming in the prediction of biological processes such as algal metabolism. The DDM is trained with measured data, and then predicted concentrations of Chl-a (response variable) with new input predictor variables (water temperature, $\mathrm{PO}_{4}-\mathrm{P}$ and total phosphorus) are generated from physically-based model. The results are better than those of the CE-QUAL-W2 model. Although the DDM itself is not a time series model, it generates time series data when it predicts 
using time series input data. The hybrid model complements the separate use of the physically-based model and the DDM, and gives improved results. It can therefore be used for forecasting and prediction of water quality for decision making.

\section{6) Decision support}

The CE-QUAL-W2 model calculates water quality at each point of the reservoir according to the nutrient reduction scenario in the watershed, and then the DDM predicts the Chl-a concentrations as a time series with the generated data from the CEQUAL-W2 model. Also, a short-term forecast is executed successfully by an exponential smoothing method.

This study presents a systematic approach to water quality assessment, hybrid modelling and decision support for in-reservoir eutrophication management. A datadriven model using clustering and PLSR implemented in this study predicts successfully Chl-a concentrations. The surface water discharge of the Yongdam reservoir induces a transfer of pollutants from the middle layer to the surface layer. This nutrient transportation mechanism is recommended to limit nutrient accumulation on the bottom of the reservoir, but it also offers an opportunity for a continuous algal production during thermal stratification in the surface water. However, the micro-scale turbulent mixing and its impact on nutrient uptake by algae, toxic compounds and weather conditions etc. are analysed for further understanding of algal metabolism in the reservoir.

Reservoir water quality shows very often temporal and spatial fluctuations. For further understanding and future study, the most important and difficult issues are the reservoir water sampling and analysis of the sampled water quality. Thus the data should be collected with a shorter time interval and at smaller spatial distances to reflect water quality variations in the entire reservoir and to be pertinent for each study objectives.

Finally, it is expected that this study will contribute to the development of tools for reservoir water quality management and/or a predictor of environmental processes. 


\section{Acknowledgements}

My sincere gratitude goes to my promoter, Professor Roland K. Price for offering me the opportunity of Ph.D. study in the hydroinformatics department at UNESCO-IHE. It would have been impossible for me without his kind teaching, encouragement and awakening my spirit for scientific thinking instead of the engineering I was accustomed to. I will always cherish the meetings filled with his pointed questions, my weak answers, his clear explanations, and his concern for my family as precious memories.

Mr. Keun-Ho Kim, President of K-water and Dr. Kyul-Ho Kwak, former President of $\mathrm{K}$-water, supported me in all things related to my study. I offer my gratitude to them. I also appreciate Dr. Seok-Ku Ko, former President of K-water, who suggested Ph.D. study to me. I appreciate his support to me, his great passion for K-water and water worldwide.

I was happy to be taught by three mentors. I am very thankful to Professor Dimitri Solomatine, Dr. Peter Keldman and Dr. Iona Popescu. They provided many theories and materials to me about data-driven modelling, reservoir water quality and hydraulic mixing of the reservoir. Technical discussions with them, their understanding to my study and kind guidance for a couple of my research aims was sufficient for hybrid modelling and the water quality assessment of the thermal stratified Yongdam reservoir, and a driving force for my long research endeavor.

The assistance of the research team of the Yongdam reservoir in sampling reservoir water and analysing the sampled water was a great help to my study. Dr. Jae-Ki Shin supplied me with various insights related to reservoir eutrophication and bioassay, the discussion with him used to keep us in my office until midnight, and continue at the beautiful café, 'Waba', which means come and see, with nice a cup of juice, on the way to his lodgings. During my stay in Korea, Dr. Hye-Keun Lee, Dr. Noh-Seok Park, Mr. Sang-Uk Lee, Mrs. Sun-A Jeong, and Professor Meung-Geun Chun of Chungbuk National University were my unforgettable supporters. Discussion with them was very useful and provided me with a paradigm of modelling. I would like to express my thanks to all them.

I am very thankful to all staff members of the department of Hydroinformatics and Knowledge Management at UNESCO-IHE. The greetings with the head of the department, Ir. Jan Luijendijk in the early mornings during my thesis writing were like a vitamin to start the day. Whenever I met Dr. Andreja Jonoski, he called my name with kindness. I felt I was a member of the Hydroinformatics department. Thank you so much Dr. Jonoski. Also, Ms. Jolanda Boots was my supporter at UNESCO-IHE, coordinating with K-water for my study. Thanks Jolanda.

I express my gratitude to Professor Richard Meganck, Rector of UNESCO-IHE, who visited Korea three times during my $\mathrm{Ph} . \mathrm{D}$ study. He always gave me encouragement 
whenever I met him at UNESCO-IHE and delivered my regards to all K-water staff members whom he met whenever visiting $\mathrm{K}$-water.

I cannot forget my colleagues who started their careers at the same time at $\mathrm{K}$-water. They are proud of me because of my passion for learning in my fifties. Thank you all, my friends.

Finally, my gratitude goes to my two sons, Hyo-joon and Hyung-joon, and my wife, Hyun-sook. Their sacrifices allowed me to successfully finish my Ph.D study. God bless you and I love you forever. 


\section{Table of Contents}

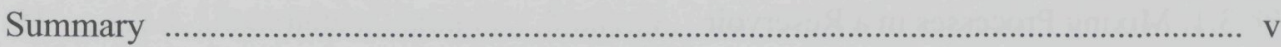

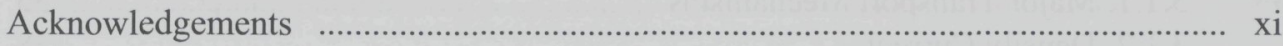

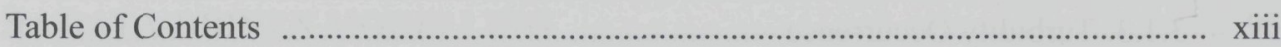

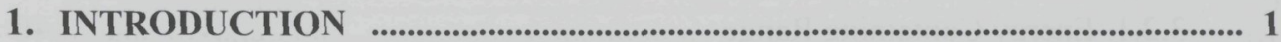

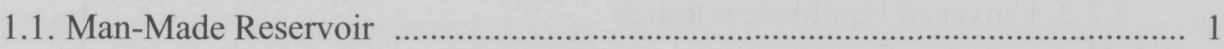

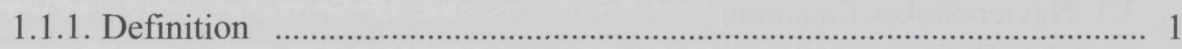

1.1.2. Amount of Reservoir in a Water ............................................................. 2

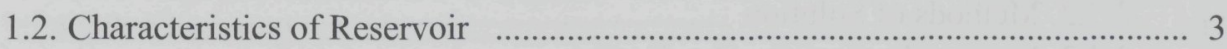

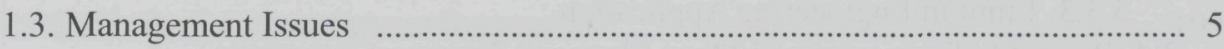

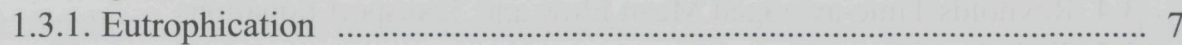

1.3.2. Turbid Density Flow ……………………………………………… 8

1.3.3. Integrated Management of Reservoir and Watershed ………………..... 8

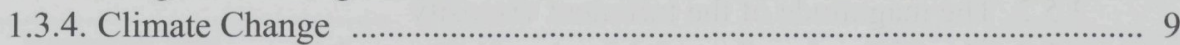

1.3.5. Eco-Hydraulic Modelling ....................................................................... 10

1.4. Framework of Reservoir Management …………….................................... 11

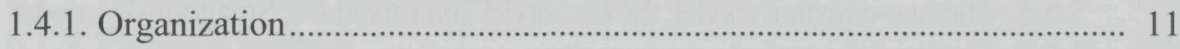

1.4.2. Environmental Quality Objectives and Standards ………………….... 14

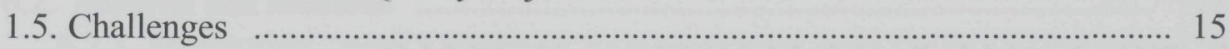

1.5.1. Eco-Hydrodynamic Modelling ……………………………………...... 15

1.5.2. Data-Driven Modelling ……………………………………………….... 17

1.5.3. Decision Support System ………………………………………..... 18

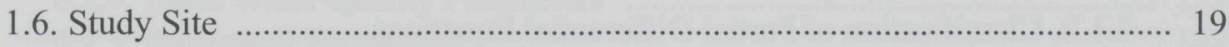

1.7. Study Aim, Objective and Hypothesis ………………………………........ 20

1.8. Layout of the Thesis …………………………………………………….... 21

2. RESERVOIR ECOSYSTEMS _.................................................................. 23

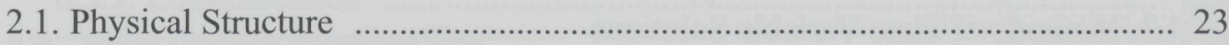

2.1.1. Longitudinal Zonation ........................................................................... 23

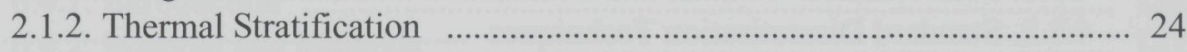

2.1.3. Reservoir Morphology ………………………………………........ 25

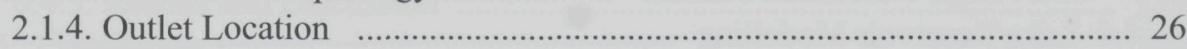

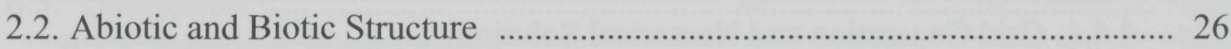

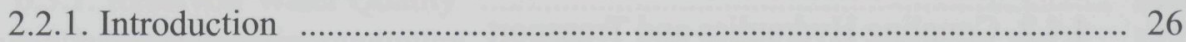

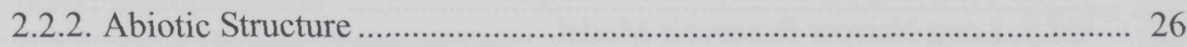

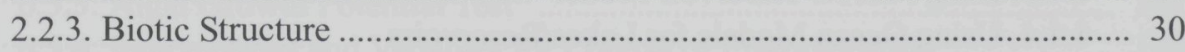

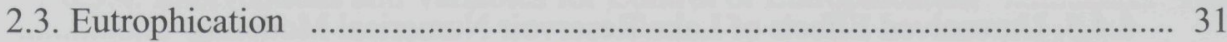

2.3.1. Assimilation and Limiting Nutrient ………………………………..... 31

2.3.2. Environmental Factors ………………………................................. 33

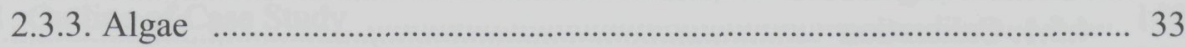


3. PHYSICAL PROCESSES OF RESERVOIRS .................................................... 35

3.1. Mixing Processes in a Reservoir …………………................................. 35

3.1.1. Major Transport Mechanisms .............................................................. 35

3.1.2. Density Current ………………………………………………….... 38

3.1.3. Turbidity Occurrence ………………………………………………... 44

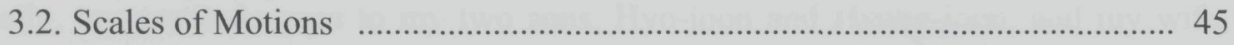

3.2.1. Energy Containing Range ......................................................................

3.2.2. Universal Equilibrium Range .............................................................. 47

3.3. Navier-Stokes Equation ……………………………………………........ 50

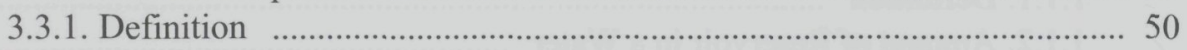

3.3.2. Methods of Solution ..................................................................... 51

3.3.3. Limit in Engineering Application …………………………………….. 54

3.4. Reynolds Time-averaged Mean Flow and Transport Equations ………….... 55

3.5. Turbulent Viscosity ................................................................................. 57

3.5.1. The concept of turbulent viscosity (Eddy Viscosity) ............................ 57

3.5.2. The magnitude of the turbulent viscosity ………………………………. 59

3.5.3. The mixing length model (zero-equation model) .................................... 60

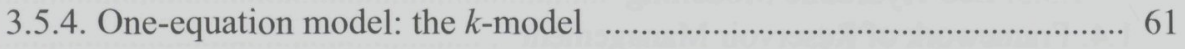

3.5.5. The two-equation model: the $k-\varepsilon$ model and mixed $k-\varepsilon$, Smagorinsky model ... 62

3.5.6. Limit of the turbulent-viscosity concept …………………………….... 64

\section{PHYSICALLY-BASED NUMERICAL MODELLING …................................. 65}

4.1. Equations for Physical Process ………………………………………….... 65

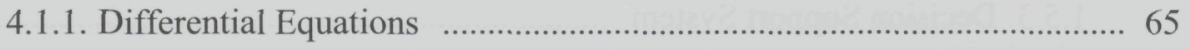

4.1.2. Classification of Partial Differential Equations ...................................... 66

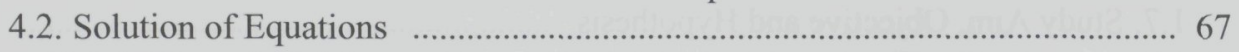

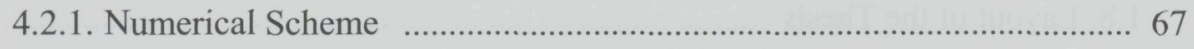

4.2.2. Explicit scheme for Hyperbolic PDEs ………………………........... 68

4.2.3. Implicit schemes for Hyperbolic PDEs ...………………………....... 70

4.3. Methods of Time Variable Solutions ………………………………..... 70

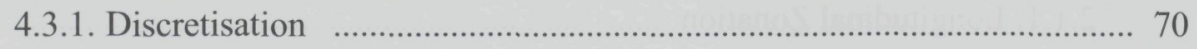

4.3.2. Standard Discretisation Techniques …………………………........... 71

4.3.3. High-Resolution Scheme .................................................................. 72

4.4. Lake/Reservoir Modelling ………………………………………….... 75

4.4.1. Grid Generation and Numerical Solution ............................................. 75

4.4.2. Coupling Hydraulics and Transport …………................................... 77

4.4.3. Long Narrow Reservoir Model …………………………………....... 78

4.4.4. Using Ocean Models for Lakes/Reservoirs ………………………..... 78

4.4.5. Unresolved Effects of Lake/Reservoir Numerical Modeling ................ 80

4.4.6. Integration of Environmental Models …………….............................. 83

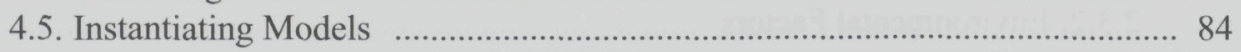

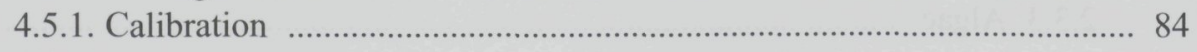


4.5.2. Verification \& Validation vs. Confirmation ….................................... 85

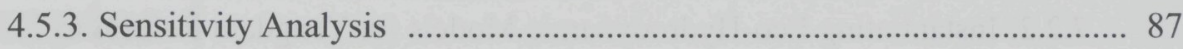

4.5.4. Uncertainty/Variability Analysis ...................................................... 89

4.5.5. Quality Assurance ........................................................................ 94

5. DATA-DRIVEN MODELLING ……....................................................... 95

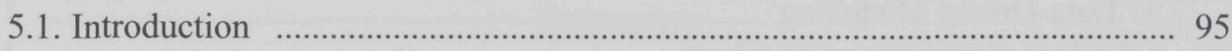

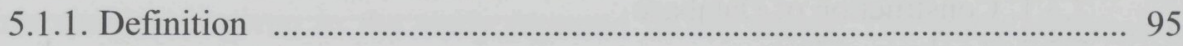

5.1.2. DDM and Science ………………………………………………….... 96

5.2. Machine Learning Methods ……………………………………………...... 97

5.2.1. Regression and Partitioning …………………................................ 98

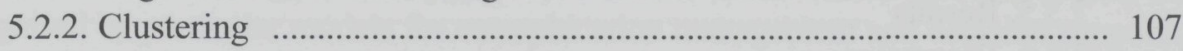

5.2.3. Partial Least Square Regression ........................................................... 109

5.3. Application of DDM to Eco-environment ……………………………...... 110

6. CASE STUDY: DATA ACQUISITION \& ASSESSMENT …........................ 113

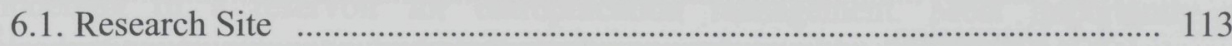

6.1.1. Geographic and Limnological Features ………................................ 114

6.1.2. Hydrologic and Meteorological Characteristics ................................... 116

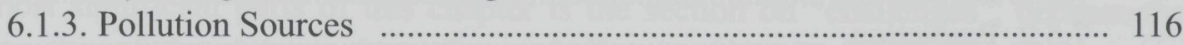

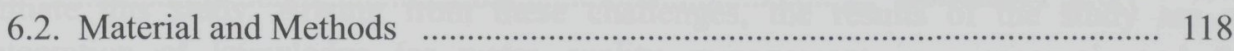

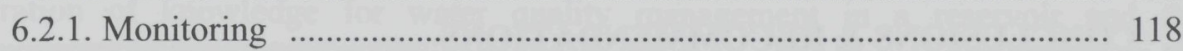

6.2.2. Hydrological and Meteorological Measurements ................................ 118

6.2.3. Water Temperature and Thermal Stratification ................................... 119

6.2.4. Basic Water Quality Parameters ........................................................... 121

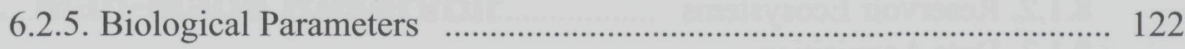

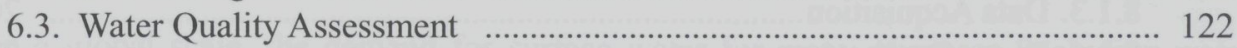

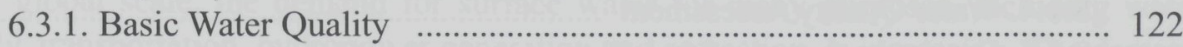

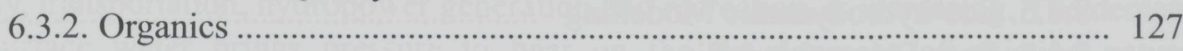

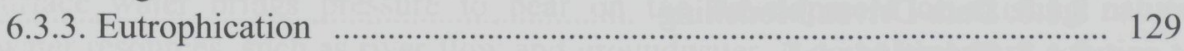

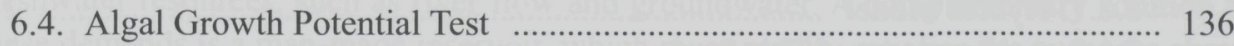

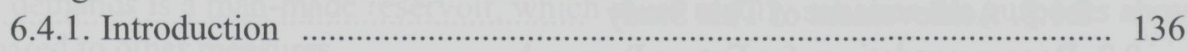

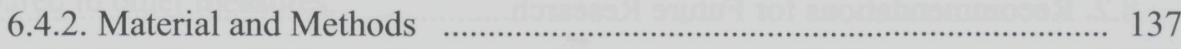

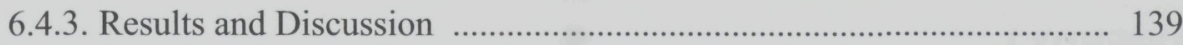

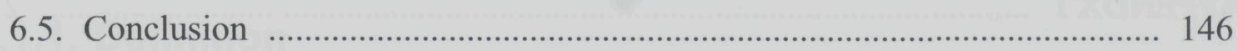

6.5.1. Reservoir Water Quality .............................................................. 146

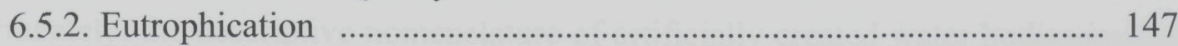

6.5.3. Algal Growth Potential Test ......................................................... 147

6.5.4. Interventions and Variables for Control of Eutrophication ................. 148

7. CASE STUDY: CONSTRUCTION OF MODELS ......................................... 149

7.1. Outline of Case Study ............................................................................ 149

7.2. Eco-hydrodynamic Modelling …………………………………………....... 151 
7.2.1. Model Selection ……........................................................................ 151

7.2.2. Instantiating Eco-Hydrodynamic Models for Yongdam Reservoir ...... 155

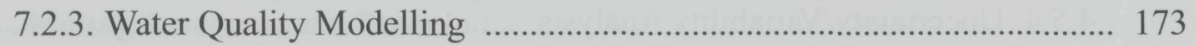

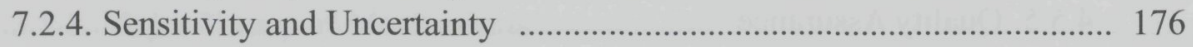

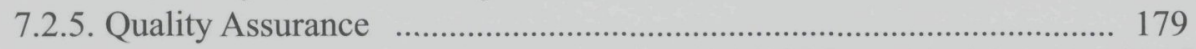

7.2.6. Discussion and Time Series Analysis …………………………….... 180

7.3. Data-Driven Modelling ……………………………………………... 182

7.3.1. Construction of Database …………………….......................... 182

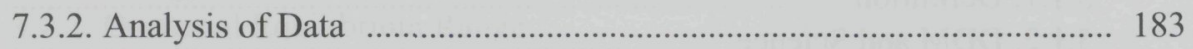

7.3.3. Selection of Task-Relevant Model ...................................................... 185

7.3.4. Construction of Data-Driven Models ……………………………...... 185

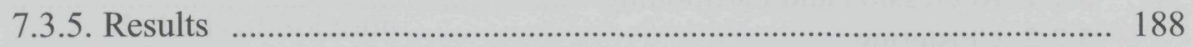

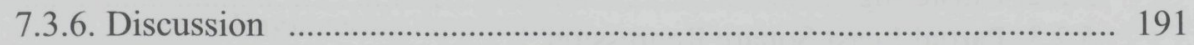

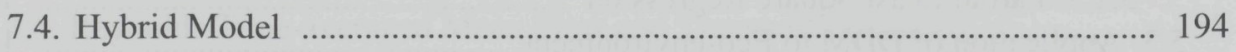

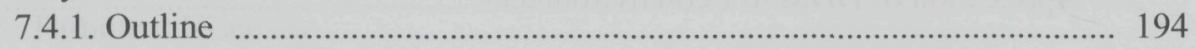

7.4.2. Integration of Physically-Based and Data-Driven Models ................. 195

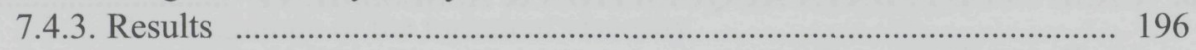

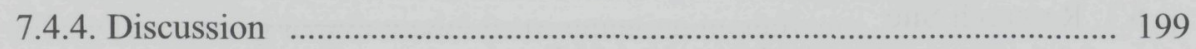

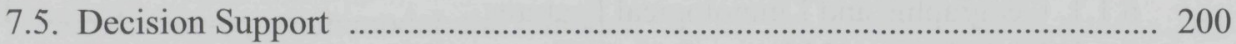

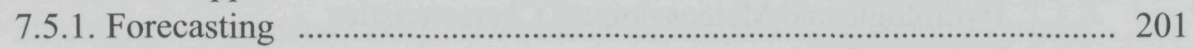

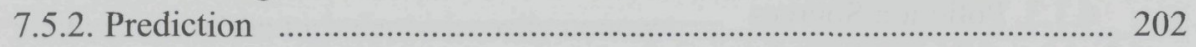

8. CONCLUSIONS \& RECOMMENDATIONS ................................................. 205

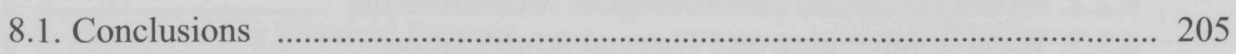

8.1.1. Reservoir Water Quality Management …………………………….... 205

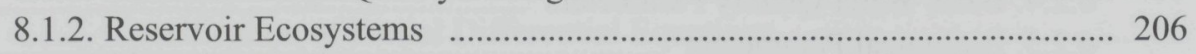

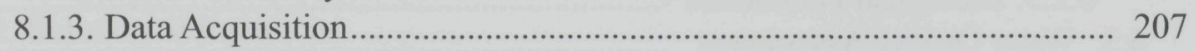

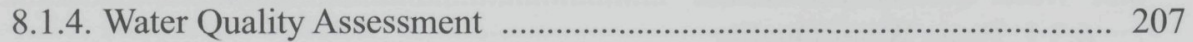

8.1.5. Eco-hydrodynamic Modelling ........................................................... 208

8.1.6. Data-Driven Modelling ……………………………………………...... 209

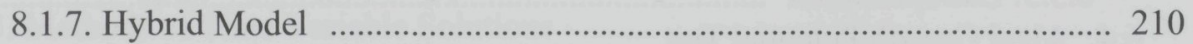

8.1.8. Achievement of This Study …………………………………….... 210

8.2. Recommendations for Future Research...................................................... 211

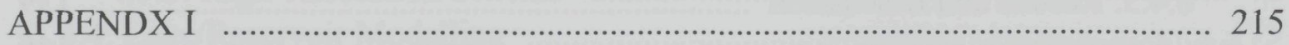

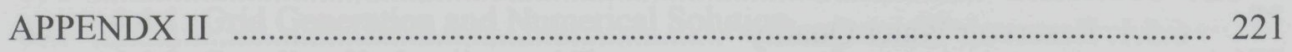

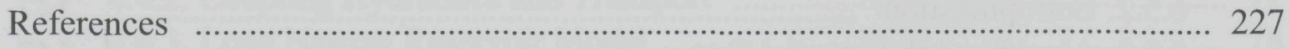

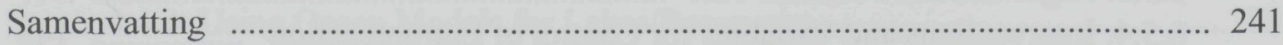

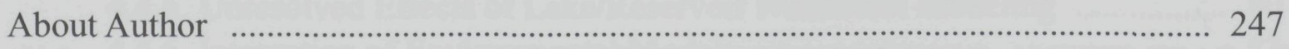




\section{Chapter 1}

\section{INTRODUCTION}

\section{Summary}

An introduction is given to the study reported in this thesis. The study concerns the management of eutrophication and the associated setting Environmental Quality Objectives (EQOs) and Environmental Quality Standards (EQSs) in a deep reservoir. The reservoir has significant physical and chemical characteristics, which are recognised by particular models for eutrophication management and decision support. A description is provided of the study site, and the management issues associated with the Yongdam reservoir. The chapter also explains the study issues of reservoir management, the reasons for using physically-based numerical models, and the need for water quality assessment and data-driven modelling techniques to generate data for integrated modelling of a reservoir for eutrophication management. Most importantly an introduction is given to hydroinformatics, together with the role and function of hydroinformatics in facilitating reservoir eutrophication management processes. One of the most significant parts of this chapter is the section on "challenges", because they initiate this study. Arising from these challenges, the results of the study are an integration of knowledge for water quality management in a reservoir and the improvement of data-driven modeling techniques to be used in reservoir modelling.

\subsection{Man-Made Reservoir}

On a global scale, the demand for surface water for many purposes, including water supply, transportation, hydropower generation and recreation, is increasing. The demand for surface water brings pressure to bear on the development of existing natural freshwater resources, such as river flow and groundwater. A complementary solution to these demands is a man-made reservoir, which more readily satisfies the purposes above compared to other measures.

\subsubsection{Definition}

Unfortunately, the descriptive nomenclature of artificially created water bodies is often confusing. The term 'dam' can be applied to both the physical structure retaining the water, and the water so retained. In this thesis 'dam' will be used solely to describe the physical structure and the term 'reservoir' will be used to denote the artificially created water body.

A reservoir is therefore:

- a water body contained by embankments or a dam, and subsequently managed in 
response to specific community needs; or

- any natural waters modified or managed to provide water for developing human activities and demands (Thornton et al., 1996).

In most cases, a reservoir as a water resource is often developed in those regions of the world where it is necessary to find a solution to recurring problems of water shortages (drought) or excess floods and where there is a lack of substantial numbers of natural lakes (ILEC, 2005). This is often the case in areas where there are significant seasonal variations in precipitation. For example, the characteristic of precipitation in Korea is that two thirds of the yearly rainfall is concentrated during the summer monsoon from June to September. Therefore, runoff during the summer monsoon has to be minimized for flood control and yet stored in the reservoirs for water supply during the drought season.

Reservoirs vary in size from a pond to a large lake, but the range of reservoir types and morphological variation related to natural lakes is in general much greater. For example, both the regular and irregular water bodies are likely to be reservoirs. This variability in reservoirs, allied to management intervention, ensures that their water quality and process behaviour is even more variable than may be characterised as limnologically normal (Thornton et al., 1996).

In spite of differences here and there, lakes and reservoirs in most cases are studied synonymously as water bodies having the same physical properties. This is accepted in this study.

\subsubsection{Amount of Water in a Reservoir}

The amount of water in reservoirs is a very small part, about $0.0003 \%$, of the global amount of water (Figure 1.1). Reservoir water is a very limited resource, and a valuable water source, because it is developed due to a strong and inevitable need.

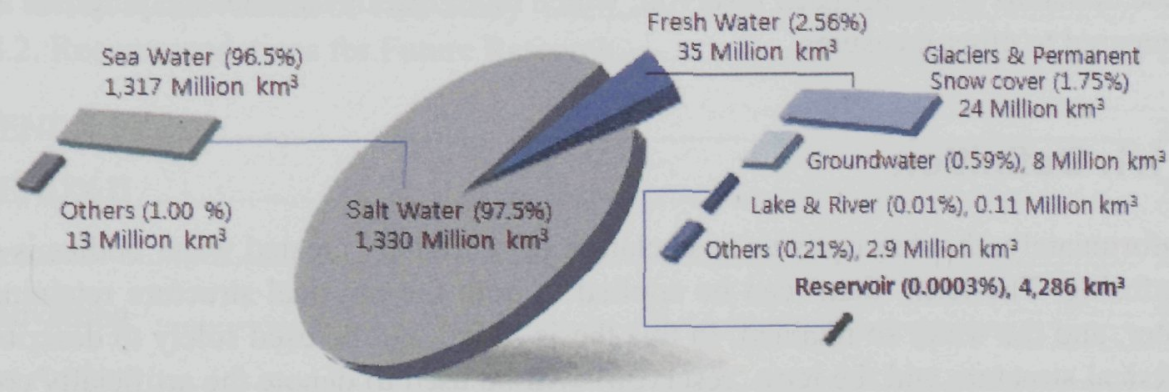

Figure 1.1. Global water and freshwater estimate (Source: http://www.unep.org/vitalwater/).

Although local water shortages have been reduced to a certain extent through the 
construction of reservoirs, the degradation of reservoir environments has already became a world-wide issue. Freshwater from reservoirs is an essential, but scarcely renewable natural resource, which supports life and promotes socio-economic development. Water can be a natural resource when it sustains a suitable quality for each use. Therefore the state of a reservoir's water quality or any water quality pollution will interfere with vital and legitimate water uses (Meybeck et al., 1992). Due to human settlements and misuse of surrounding land, such as anthropogenic use of ecosystems in a drainage basin, increased pollutant inflows can dramatically accelerate the process of eutrophication. This process has been diminishing the utility value of a large number of reservoirs.

\subsection{Characteristics of Reservoir}

A river consists of a flow of water in a channel, but a reservoir is a stock of water impounded normally behind a dam. As a result, the reservoir eco-environment is totally different from the original river eco-environment. In most instances, the peculiar form of a reservoir, its mode of operation, and an unnatural location and shape may cause considerable, actual variations in the basic limnological behaviour (Straskrabá et al., 1993). The reservoir will also change the pattern of flows released downstream. There should be a lively debate as to whether the social and environmental impacts outweigh the benefits before a dam is constructed. These issues are outside the scope of this study.

Reservoirs are therefore as important as lakes and rivers and have great value in water usage, but reservoirs should not be considered as having necessarily a unique ecosystem, because their long retention times can be assumed to be similar to those of lakes. Canfield and Bachmann (1981) suggest that lakes and reservoirs represent a continuum of ecological conditions. Kimmel and Groeger (1984) and Soballe and Kimmel (1987) view this continuum as one ordered by water residence time, which can be determined as the reservoir volume divided by the annual discharge. Their work indicates that reservoirs and natural lakes with similar residence times have similar ecological attributes. Despite many of the similar features of reservoirs and natural lakes, reservoirs can be different from lakes where there are various changes in reservoir operation.

\section{1) Thermocline}

The thermal variations in the reservoir are caused by heating due to solar radiation during the daytime and to the unique thermal properties of water, of which density is inversely proportional to temperature under natural conditions. The greatest density of water is at $3.98^{\circ} \mathrm{C}$. Figure 1.2 shows the structure of a stratified, temperate lake.

Usually sunlight is intensive between late spring and early autumn, and can penetrate the water depth by as much as 10 meters. During this period, the solar radiation heats the upper layer of the water in the reservoir, and stratification develops due to the difference in density between the upper layer (epilimnion) and the lower layer 
(hypolimnion).

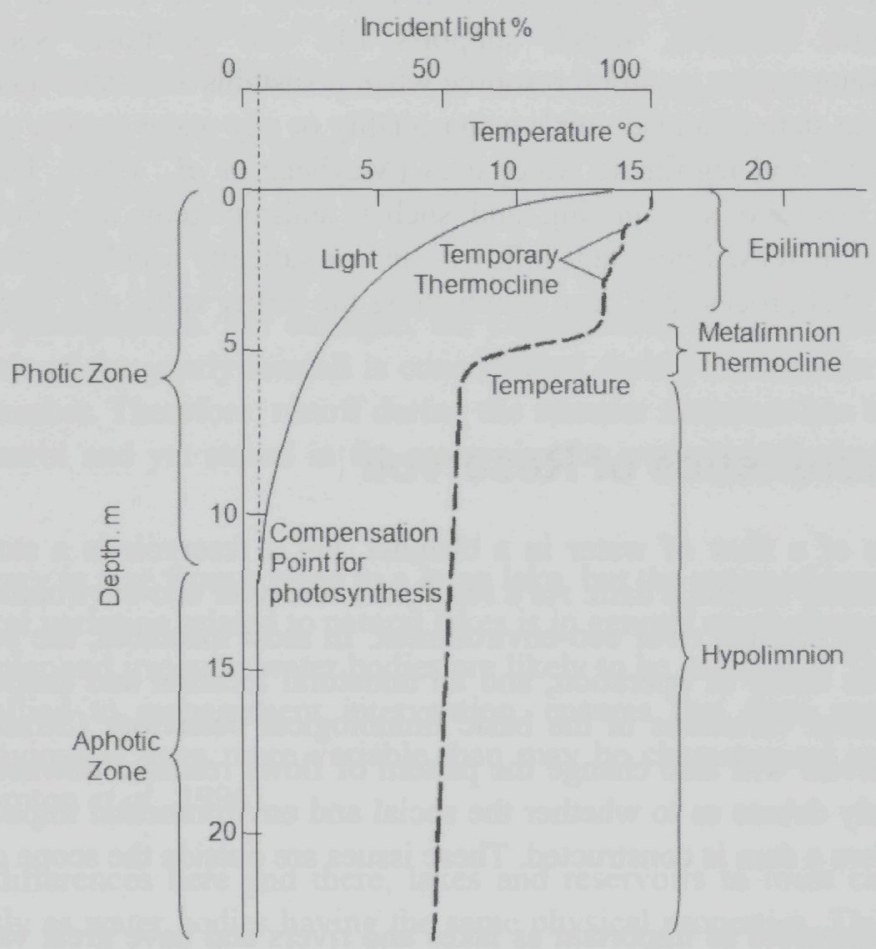

Figure 1.2. Structure of a stratified temperate lake. The temporary thermoclines are caused by heating on calm days; they are destroyed each night by convective cooling or by wind. The rather low transparency indicates that the lake is eutrophic (high biomass) (Source: Goldman and Horne, 1983).

The boundary layer (metalimnion) situated between these two layers can have a very well defined thermocline where the temperature decreases with depth. The actual position of the thermocline also depends on the wind stress experienced at the surface of the water (Birge, 1897).

For these reasons, most reservoir hydrodynamic modellers use stratified water temperature profiles to calibrate their models.

\section{2) Chemical Characteristics}

The chemical quality of the aquatic environment varies according to local geology, the climate and the amount of soil cover, etc. If surface waters were totally unaffected by human activities, global freshwater, depending on the variable of interest, would have natural chemical concentrations suitable for aquatic life and most human uses (Meybeck and Helmer, 1996). But reservoirs give rise to many water quality problems, because 
there is a range of potential biological activities using chemicals that are inherent to the inflow, and which accompany other dependent chemical reactions.

With the chemical components from the watershed area, the threshold of the degradation of the reservoir water is according to the accumulation of chemicals in the reservoir due to the relatively low flow-through velocities, the development of significant vertical gradients in temperature and microbial activities in the water column.

\section{3) Biological Characteristics}

As with chemical properties, the biological characteristics of reservoirs are fundamentally the same as for natural lakes, but the peculiar physical and chemical conditions which may occur in managed reservoirs can also result in biological production and ecological successions quite different in degree and timing from an equivalent natural lake (Thornton et al., 1996).

A reservoir like a lake can be divided into zones, or communities, of plants and animals. However a reservoir does not have any littoral communities where aquatic plants are dominant due to frequent and high fluctuations in water level. Plants in the littoral zone play an important role in the overall aquatic community by reducing nutrient concentrations loaded into a reservoir, producing oxygen and providing food and shelter for insects, crustaceans, frogs, turtles and fish.

The area of open water is the home of the limnetic community. This area is the habitat of phytoplankton (algae), zooplankton (microscopic animals), and fish. The phytoplankton is very important, serving as the base of the lake's food chain and producing oxygen.

The process by which green plants (including algae) produce oxygen from sunlight, water and carbon dioxide is photosynthesis. A pigment, chlorophyll, produced by the plants facilitates this process. Since sunlight is very important to photosynthesis, oxygen will be produced only as deep as the sunlight penetrates. Below the limnetic zone is the profound community, where light does not penetrate. This zone or community is dominated by respiration, or oxygen consumption, rather than oxygen production. This zone corresponds roughly to the hypolimnion layer. The community in this zone consists of such organisms as bacteria and fungi. These organisms break down or consume (decompose) dead plants and animals that settle out of the waters above. This process consumes oxygen.

\subsection{Management Issues}

When dams were first constructed, reservoir managers began to secure their water resources through the reservoirs that were formed, and to increase the operating efficiency of facilities for various uses. This meant that initial reservoir management was mainly focused on hydrological events to control reservoir water quantity for water 
supply and flood control. With continuing development of reservoirs, management issues moved from water quantity to water quality. Reservoirs in densely populated or agricultural areas, which receive little management control, have a tendency to be highly enriched with nutrients, although most have a rapid flushing regime. There is, therefore, a potentially high dependency of productive capacity on management regime, source water qualities, and internal chemical and biological processes.

Reservoir water quality management is defined as planning for the protection and improvement of the reservoir water quality for various beneficial uses. It requires an appropriate management organization and the scientific background associated with integrated watershed management. Over the past twenty years, a strong global consensus has begun to develop that the watershed is, in fact, the best unit for the management of water resources. Now, countries all over the world try to place water management actions in the context of natural and human systems, namely the watersheds and their human communities. Therefore a sound integrated watershed management needs a rationally, well-organized framework among specialists in widely varying disciplines - engineering, bio-chemical aquatic sciences, economics, sociology, law and ethics, and among government agencies, private industries, nongovernment organizations, and the public (Heathcote, 1998).

On the other hand, it is true that the environmentally best management practice involving costly physical/biochemical treatment technologies is nearly always impossible within the limit of budgetary constraints. Therefore, tools are needed to understand what results are brought about by well-established management strategies. Water quality modelling deals with the development and application of mathematical models to integrate present understanding of the dynamics of materials carried by the flow and to predict the fate of these materials in the natural environment (Martin and McCutcheon, 1999). In so doing, the relations between the forcing functions on the reservoir and the state variables or reservoir response to impacts can be found or demonstrated. This knowledge can then be used to control the forcing function, if possible, to achieve the desired state of the reservoir (Jørgensen and Vollenweider, 1988); see Figure 1.3.

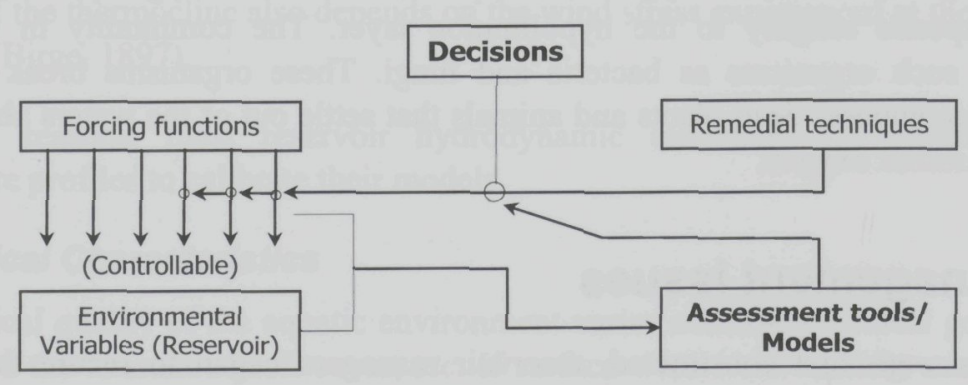

Figure 1.3. Function of assessment tools/models in decision making (Jørgensen and Vollenweider, 1988). 
Due to the inadequacy of the traditional water quality monitoring programmes to fully describe the hydrodynamic behaviour of a reservoir, it is true that water quality managers have sought refuge in modelling. Most of the analytical thinking about assessment methodologies revolves around models (Wilson and Walker, 1989Buras, 2001; Buras, 2001).

There are many management issues for reservoir communities: integrated watershed management for water quantity and quality control, best management practices, management tools, management organization, etc. Management issues to be described in this study are focused on theoretical fundamentals related to an understanding of the reservoir environment for its communities.

\subsubsection{Eutrophication}

The term 'eutrophication' originates from the slow aging process during which surface water in a lake, estuary, or bay evolves into a bog or marsh and eventually disappears with the accumulation of biomass through plant growth and decay (UNEP and PHO, 2005). Nowadays the term refers to the enrichment of an aquatic system by addition of nutrients which bring about the ecological changes that result. Generally, it is the natural or man-induced process by which a body of water becomes enriched in dissolved nutrients, particularly phosphorus, that stimulate the growth of aquatic plants and enhance organic production of the water body, and so eutrophication describes the biological effects of an increase in the concentration of nutrients. Excessive enrichment may result in the depletion of dissolved oxygen by the degradation of aquatic plants and eventually to species mortality and their replacement.

The principal environmental problem associated with the degradation of reservoir water quality is therefore eutrophication. Scientifically speaking, eutrophication is only a special aspect of reservoir productivity. Reservoir productivity is, on the one hand, the result of the physical and chemical interactions within the catchment system where the reservoir is located. On the other hand, it is a function of the internal physical, chemical and biological dynamics of the component compartments of the reservoir itself. Sunlight availability, water availability, temperature and the supply of plant nutrients are the four most important factors determining the Net Primary Production (NPP) by photosynthesis. Altered availability of nutrients affects the rate of primary production in all ecosystems, which in turn changes the biomass and the species composition of communities.

In freshwater aquatic systems, a major effect of eutrophication is the loss of the submerged macrophyte community. Following eutrophication, the sunlight is intercepted by the increased biomass of phytoplankton exploiting the high availability of nutrients. In principle, the submerged macrophytes could also benefit from increased nutrient availability, but they have no opportunity to do so because they are shaded by the free-floating microscopic organisms. Reservoirs in this case have one more reason for the loss of the submerged macrophyte community, which is large and steep 
fluctuation of water level. These fluctuations induce no rooted-vegetations at littoral zones and surrounding boundaries.

\subsubsection{Turbid Density Flow}

Turbidity, in simple terms, refers to how clear the water is. It is the amount of total suspended solids (TSS) of micro-scale in the water - the murkier the water appears, the higher the turbidity. Inorganic and detrital particles from the watershed vary largely in response to hydrological events such as storms and snowmelt. This turbid water (possibly with more dense water due to lower temperatures) occurs as density currents in thermally stratified reservoirs. Particulates of clays and silt remaining in dense water without settling because of Brownian movement are spread in the whole of the reservoir during the turnover process.

The reason why turbid dense flow in the reservoir becomes an issue of management is that it relates to water quality changes. In practice, a density current moves at a slow speed, and yet is able to transport high turbidity loads for distances over a few tens of kilometres through a reservoir in Korea. The effect of this is that the flow of the density current also seriously impacts the nutrient transport and diffusion processes until the start of destratification in the water column. A density current can easily jump over obstacles in its path, due to the low relative gravity. Therefore turbid water in a longnarrow meandering reservoir can flow vertically upwards. For these reasons, one of the most important objectives for reservoir management is to control the thermal stratification effectively.

\subsubsection{Integrated Management of Reservoir and Watershed}

The term 'watershed' means a geographic area bounded peripherally by a conceptual line from which water flows away from it and such that the water in the area drains to a common outlet. Therefore, the majority of the nutrient and sediment loads to reservoirs come from their associated watersheds, and the loads are closely tied to the soil component, vegetation, hydrometeorologic and land use conditions, industrial production activities etc. Most reservoir tributaries convey nutrients and sediments from the watershed without any improvement to their water quality. Thus reservoir water quality is affected by the watershed and the limited waste assimilation capacity of reservoirs (see Figure 1.4); a strategy for managing water quality in a reservoir should first address the management of the watershed.

Over the last couple of decades, reservoir management has focused on integrated watershed management which is, in fact, the best unit for the management of water resources. Now, many countries in the world try to place water management actions in the context of natural and human systems, namely the watersheds and the human communities that are present. 
A sound integrated watershed management needs a rationally well-organized framework among specialists in widely varying disciplines, including engineering, bio-chemical aquatic sciences, economics, sociology, law and ethics, and among government agencies, private industries, nongovernment organizations, and the public (Heathcote, 1998). This study is focused on the response of a reservoir to its watershed as a part of the integrated management of the Yongdam reservoir.

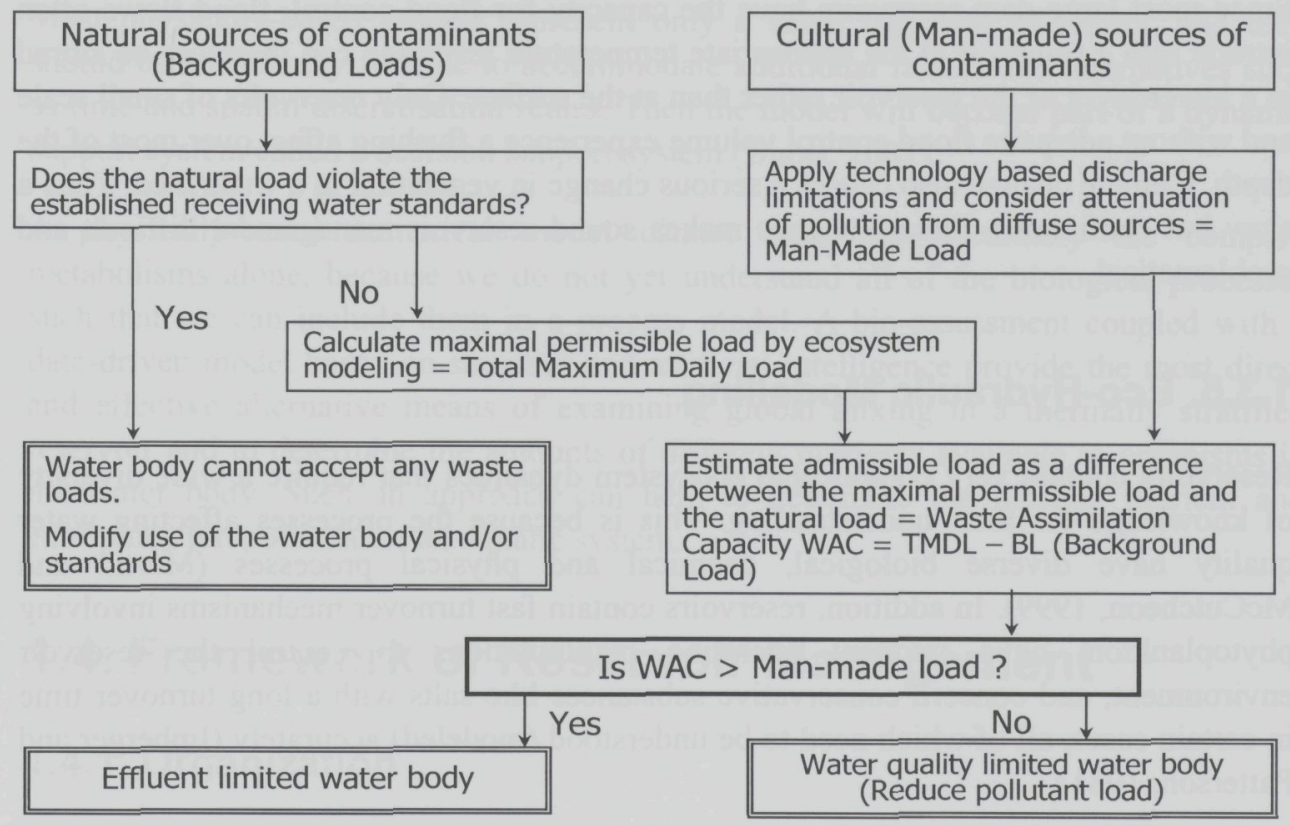

Figure 1.4. Concept of waste assimilation Capacity (Novotny, 1995).

\subsubsection{Climate Change}

Long term changes to the earth's climate are continually taking place. However, there is significant concern that some current changes are directly attributed to human activity. Global climate change is expected to affect both the quantity and quality of water resources. It will affect particular elements of the hydrological cycle, changing river discharges, and hence water retention times in reservoirs. It is predicted that the timing and intensity of floods and droughts will also change. Since water is the main medium which is responsible for the export of nutrients and pollutants from catchments, the above processes will alter nutrient transport patterns to fresh waters, and hence their physical and chemical parameters (Kaczmarek, 2004).

Global warming causes enhanced flood flows due to snowmelt, and significant changes in annual precipitation in time and space. It will be difficult in many cases to decide whether these changes will impact affirmatively or negatively on reservoir water quality. 
However, the most critical impact on reservoirs is on water quantity, with the possibility of too little water for supply or too much, leading to a failure of flood control and even the prospect of a dam break. They can also have serious sociological and economic consequences. Flood flows radically change reservoir ecosystems compared to their state before the flood events. In this case, there is a choice between two kinds of flood control mechanisms: retain or discharge the flood water.

Since most large dam reservoirs have the capacity for flood control, flood flows often intrude into a reservoir at the appropriate temperature level and can therefore be stored in a lower layer of the reservoir rather than at the surface. Only reservoirs of small scale and without adequate flood control volume experience a flushing effect over most of the depth. Climate change also causes a serious change in vegetation in a watershed. Thus a slow but continuous climate change makes sound reservoir management difficult and problematical.

\subsubsection{Eco-Hydraulic Modelling}

Reservoirs contain very complicated ecosystem dynamics that require a wide diversity of knowledge for their understanding. This is because the processes affecting water quality have diverse biological, chemical and physical processes (Martin and McCutcheon, 1999). In addition, reservoirs contain fast turnover mechanisms involving phytoplankton, have frequent operating manipulations to control the reservoir environment, and concern conservative substances like salts with a long turnover time in certain cases, all of which need to be understood (modeled) accurately (Imberger and Patterson, 1981).

Since the hydrodynamics of the reservoir affects all processes, whether in the short-term or the long term, the hydrodynamics should be described at all scales. In other words, a water quality model should be based on the mixing processes of small scale motions, which impact on not only nutrient advection and diffusion but microbial activities, as they are superimposed on the large scale mean motions (Hodges et al., 2000). Traditionally, in water quality modelling, choosing a model for a particular problem, involves adoption of proper time and space scales as a crucial first step. Then a model is developed or chosen that can describe the physical processes at this scale. The dynamics of water quality processes that influence the trophic status of a reservoir or lake can be considered over four different time scales: very short (days), short-term (months), and long term (few years), and very long term (climatological time scale). The processes should be adequately described at all these scales to obtain an intensive understanding of the reservoir behaviour.

In water quality simulations of reservoirs, situations arise where a full three dimensional simulation is required, especially in cases of reservoirs with sinuous convoluted morphometries. In such cases, 3D modelling of the reservoir is required over seasonal and annual time scales. This is done to provide better resolution of topographic effects, 
internal waves, mixing, and spatial gradients of environmental forcing, as required by a detailed hydrodynamic description of a reservoir.

Unfortunately, a 3D simulation of lake dynamics over an annual time scale requires substantial computational time and memory when applied on a personal computer. Therefore, presently, three dimensional models are applied over short time scales to gain greater understanding in the transport processes where necessary (Hodges et al., 2000). Most physically-based models represent only a small fragment of reality, and they should be sufficiently flexible to accommodate additional factors and alternatives such as time and spatial discretisation scales. Then the model will become part of a dynamic support system called a decision support system (Buras. 2001).

A physically-based numerical model cannot reproduce accurately the complex metabolisms alone, because we do not yet understand all of the biological processes such that we can include them in a process model. A bio-assessment coupled with a data-driven model based on statistics and artificial intelligence provide the most direct and effective alternative means of examining global mixing in a thermally stratified reservoir and to determine the amounts of different nutrients available to organisms in the water body. Such an approach can help to determine the limiting nutrient and eutrophication potential of an aquatic system.

\subsection{Framework of Reservoir Management}

\subsubsection{Organization}

A reservoir management framework can be regarded as a hydroinformatics application. Here hydroinformatics is a branch of informatics that concentrates on the application of information and communications technologies (ICTs) in addressing the increasingly serious problems of the equitable and efficient use of water for a variety of different purposes.

Growing out of the earlier discipline of computational hydraulics, the numerical simulation of water flows and related processes remains a mainstay of hydroinformatics, which encourages a focus not only on the technology but on its application in a social context. Therefore hydroinformatics draws on and integrates hydraulics, hydrology, environmental engineering and many other disciplines. It sees application at all points in the water cycle from atmosphere to ocean and in artificial interventions in that cycle such as urban drainage and water supply systems. It provides support for decision making at all levels from governance and policy through management to operations.

Hydroinformatics is network of a growing world-wide community of researchers and practitioners for reservoir management. On the technical side, in addition to computational hydraulics, hydroinformatics has a strong interest in the use of data mining that might be used with large collections of observed data for the purpose of 
data-driven modelling, for knowledge discovery, or with data generated from an existing, physically-based model in order to generate a computationally efficient emulator of that model for some purpose. In order to facilitate the understanding of this definition, ranges of hydroinformatics and related four interdisciplinary groups are illustrated in Figure1.5.

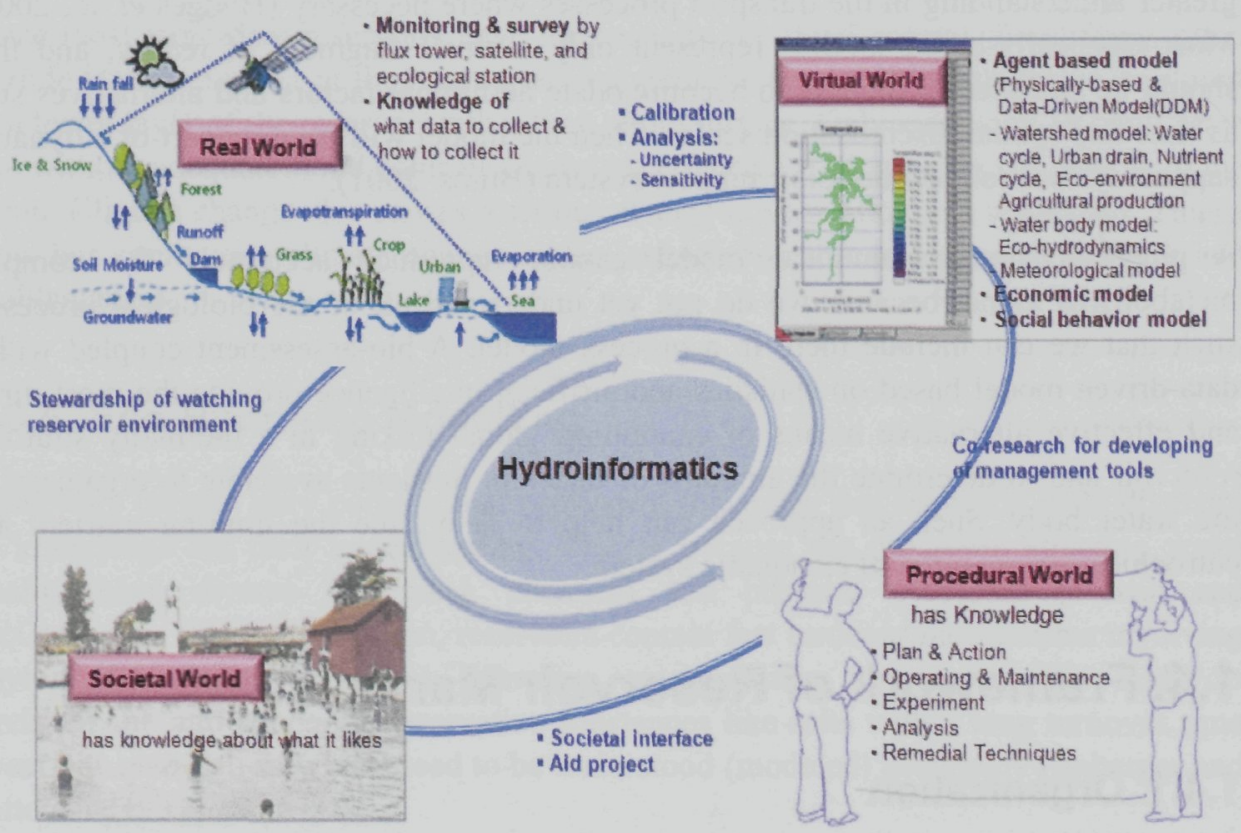

Figure 1.5. The general framework of Yongdam reservoir management.

\section{1) Real World}

The real world of the Yongdam reservoir is enclosed within a drainage boundary, also called the Catchment or Basin having only two outflow points: downstream at the dam site and the intake for drinking water sources. It gives us data to construct the virtual world counterpart. Real time information from runoff, discharges, water level, ecoenvironment, meteorology etc. are needed for the reservoir management.

To obtain this information, monitoring and surveying by flux tower, satellite and ecological station should be executed with time interval and spatial resolution representing the real site. Knowledge of what data to collect and how to collect it should be developed.

\section{2) Virtual World}

The virtual world is generally constructed in a computer-based simulated environment intended for its users or stakeholders to show an illusion of reality. The computer accesses a computer-simulated world and presents perceptual stimuli to the user, who in 
turn can manipulate elements of the modeled world. Such simulated worlds may appear similar to the real world or instead depict imagined worlds. There are three categories in agent-based models for the virtual world: watershed, water body and meteorological model.

Basic equations are derived from the principles of conservation of mass, momentum and energy etc. for physically-based models, and the machine learning algorithm for data mining. In solving the equations for the physically-based model, data produced from the "Real World" are used for boundary and initial conditions and verification and/or validation of models. Economic and social behavior models are also components for the virtual world in hydroinformatics.

A tool is conceived in terms of computer-based models (user driven software) in the technical world; it can be defined as an integration and/or improvement of traditional simulation models for professionals with an easy-to-use interface, a feature-rich environment and a rigorous reliability. Straskrabá and Gnauck (1985) give a wideranging account of modelling in freshwater systems. It is important to realise that there is not likely to be a single "best" model or model type for a given reservoir. Experience shows that overly complex mathematical models which attempt a complete description of reservoir dynamics are invariably not used because of their extensive data demands, and their often complex nature and high relative costs of operation (Thornton, 1996).

Unlike a traditional physically-based (deductive) model, a data-driven (inductive) model is often mentioned as a decision making tool because it is easy-to-use and has less uncertainties than a physically-based model. Therefore data-driven models are more favoured by decision makers in optimizing the operating of facilities.

The development of reservoir management strategies has been greatly facilitated using a suitable management tool. Most nutrient emission control projects usually use models for an assessment of reservoir and its basin. Models used range from empirical and statistical descriptions to analytical and mathematical models.

\section{3) Procedural World}

Reservoir management should be divided between a number of technical groups according to functions related to quantity and quality and between their affiliated organizations. The reason is that inevitably there are various organizations taking part in the management activities of water quantity and quality in the watershed. In reality, the procedural world is the engine to drive forward the planning and implementation of reservoir management strategies.

The procedural world taking charge of the management of a reservoir and maintenance of various facilities should be the controller and coordinator of all sub-organizations at the watershed level. The procedural world produces all kinds of data and offers practical site knowledge to developers of management tools. The procedural world maintains an interface with the societal world and executes projects for it. 


\section{4) Societal World}

A societal world is the community relating to society and its members; social institutions, societal evolution, societal forces and social legislation. A man-made reservoir has various impacts on communities in the basis of reservoir such as a move from their settlements according to the reservoir construction and an increase of fog etc. The societal world has responsibility for watching the reservoir environment and developing agreement about what it prefers. Therefore it plays an important role in the phase of reservoir construction and its management. The procedural world has to support the societal world to practice the best management strategies.

\subsubsection{Environmental Quality Objectives and Standards}

Environmental Quality Objectives (EQOs) are non-enforceable goals, which specify targets for the reservoir water that must be met in order for the water to be considered suitable for its intended use. EQOs are generally not set by regulation (unlike Environmental Quality Standards) and are often cast in a weak form of generally desirable objectives, rather than as more concrete quantitative measures. Therefore EQOs define the aim of the environmental work.

Environmental Quality Standards (EQSs) are the allowable levels of pollutants in the water, which can produce undesired effects on the reservoir water or limits the usefulness of the water resource for present or future use. Levels may be specified as a spatio-temporal mean and/or maximum value. For certain pollutants these levels may be zero. EQSs defined for the water of the Yongdam reservoir should follow firstly national regulations (Table 1.1).

Table 1.1. Environmental quality standards of reservoir water resource for drinking water use in S. Korea

\begin{tabular}{|c|c|c|c|c|c|c|c|}
\hline \multirow{2}{*}{ Classes } & \multicolumn{7}{|c|}{ Index and Reservoir EOS } \\
\cline { 2 - 8 } & $\mathrm{pH}$ & $\begin{array}{c}(\mathrm{COD}) \\
(\mathrm{mg} / \ell)\end{array}$ & $\begin{array}{c}\mathrm{SS} \\
(\mathrm{mg} / \ell)\end{array}$ & $\begin{array}{c}\mathrm{DO} \\
(\mathrm{mg} / \ell)\end{array}$ & $\begin{array}{c}\text { Fical colifom } \\
(\mathrm{MPN} / 100 \mathrm{~m} \ell)\end{array}$ & $\begin{array}{c}\mathrm{T}-\mathrm{P} \\
(\mathrm{mg} / \ell)\end{array}$ & $\begin{array}{c}\mathrm{T}-\mathrm{N} \\
(\mathrm{mg} / \ell)\end{array}$ \\
\hline First & $6.5-8.5$ & $\leq 1$ & $\leq 1$ & $\geq 7.5$ & $\leq 50$ & $\leq 0.010$ & $\leq 0.200$ \\
\hline Second & $6.5-8.5$ & $\leq 3$ & $\leq 5$ & $\geq 5$ & $\leq 1,000$ & $\leq 0.030$ & $\leq 0.400$ \\
\hline Third & $6.5-8.5$ & $\leq 6$ & $\leq 15$ & $\geq 5$ & $\leq 5,000$ & $\leq 0.050$ & $\leq 0.600$ \\
\hline $\begin{array}{c}\text { Other } \\
\text { limits }\end{array}$ & $\begin{array}{l}\mathrm{Cd} \leq 0.01 \mathrm{mg} / \ell, \mathrm{As} \leq 0.05 \mathrm{mg} / \ell, \mathrm{Pb} \leq 0.1 \mathrm{mg} / \ell, \mathrm{Cr}^{6+} \leq 0.05 \mathrm{mg} / \ell \\
\text { Anionic surfactant } \leq 0.5 \mathrm{mg} / \ell\end{array}$ \\
& \multicolumn{7}{|c|}{ Cyanogen, Hg, Organic phosphorus, PCB: Not detectable } \\
\hline
\end{tabular}

Since the Table 1.1 shows only general standards of reservoir water resource for drinking water use in S. Korea, it may be not a description for the Yongdam reservoir. At present, an issue for the Yongdam reservoir is algal bloom that gives disgust to citizen because of taste and/or odor from decayed algae. An excessive dose of chlorine 
to the water for oxidation of algae during drinking water treatment processes can produce disinfection by-products that are harmful to human health.

For eutrophication management of the Yongdam reservoir, EQOs should include nutrients inputs that do not arouse excessive algal blooms; EQSs should do allowable algae concentration indicated by Chlorophyll-a. A warning system of algal bloom for Yongdam reservoir was implemented in three steps: 1st step 15 25mg Chl-a $\mu \mathrm{g} / \ell, 2 \mathrm{nd}$ step $25 \sim 100 \mathrm{Chl}-\mathrm{a} \mu \mathrm{g} / \ell$, 3rd step $>100 \mathrm{mg}$ Chl-a $\mu \mathrm{g} / \ell$. In this study, EQOs and EQSs are set for the maximum level of Chl-a concentration of the 2nd step that includes Chl-a concentration and algal growth potential at $5 \mathrm{~m}$ depth in the lacustrine zone of Yongdam reservoir.

Limiting factors for algal growth are weather condition, flow regime and nutrient input etc. Possible interventions and control variables to manage the reservoir eutrophication are kinds of limiting nutrient and their reduction from the watershed because of the myriad of natural forces in play, and release of nutrient out of the reservoir to a confined certain level.

\subsection{Challenges}

\subsubsection{Eco-hydrodynamic Modelling}

Spigel and Imberger (1987) state that the mixing processes in a reservoir are the sole forcing functions for the transport and movement of algal cells in a lake. Phytoplankton organisms are typically smaller than $200 \mu \mathrm{m}$. At this small scale, water turbulence has direct effects of potentially eco-physiological importance on phytoplankton: it interacts with the transport of molecules in and out of the cells, it causes mechanical cell damage and morphological adaptations, or it affects contact rates (and hence coagulation and predation processes) between suspended phytoplankters. In the case of motile cells, their trajectories in the water column are modulated by both the organism swimming behaviour and the water motion (Berdalet et al., 2005). The question arises about how exactly the mixing affects the phytoplankton dynamics.

Ecological and physiological processes are affected by turbulent flow fields on both large and small scales in eddy (energy) size. On large scales, tens of centimetres to tens of meters, turbulence affects the populations as well as individuals. Phytoplankton, other particles, and solutes are dispersed with effects on nutrient supply, light availability to phytoplankton, sediment re-suspension, and grazing by zooplankton and larvae (MacIntyre, 1998).

At the small eddy scales, less than Kolmogorov's micro length scale $(\eta)$ which are the smallest scales in turbulent flow dissipating energy, the turbulent flow field affects algae and patches of solutes directly (Peters et al., 1998). $\eta$ is typically the order of millimetres in the mixed layer. For example, the vortices of a turbulent flow field may 
distort the boundary layer around a phytoplankton cell, increasing the concentration gradient of dissolved solutes, and thereby affecting the nutrient uptake or release of wastes.

$$
\begin{aligned}
& \eta=\left(\frac{v^{3}}{\varepsilon}\right)^{1 / 4} \\
& \text { where, } v \text { is the kinematic viscosity }\left(\mathrm{L}^{2} \mathrm{~T}^{-1}\right) \\
& \quad \varepsilon \text { is the dissipation per unit mass }\left(\mathrm{L}^{2} \mathrm{~T}^{-3}\right)
\end{aligned}
$$

A positive feedback exists between phytoplankton development and reservoir hydrodynamics. An increasing amount of phytoplankton results in shallower thermoclines, warmer surface layer and other corresponding differences in hydrodynamics. However, the effect the hydrodynamics has on the phytoplankton depends on the class of phytoplankton contained in the reservoir. Therefore, before assessing the effect of hydrodynamics on the phytoplankton, the different classes of phytoplankton in the water body have to be characterized first.

An estimation of turbulent kinetic energy dissipation has enabled the inference of turbulent fluxes from the mean fields of mass, heat, and momentum. However, the direct measurement of the fluxes themselves and the inclusion in the three-dimensional hydraulic model of Kolmogorov's micro length scale have so far been difficult, and this has been an impediment to further successful theoretical development. In setting up a more complete physical frame, biologists have to approach the effects of turbulence, and try to give parameterizations to modellers without a complete understanding of the fundamental mechanisms underlying small-scale turbulence.

One of the most important eco-hydrodynamic conditions arises from the species composition in a reservoir. It is well known that the algal species are influenced by environmental conditions. The conditions are, however, changing constantly, because the forcing functions, mainly the meteorological conditions including large and small scale turbulence, density flow and thermal stratification, on ecosystems are changing rapidly and/or are time-delayed, and in addition, all the chemical and biological components are continuously changing.

Even the same species may have different properties in different environments dependent on what is most beneficial for their survival and growth. As we can see from a seasonal shift of algal species, species can only change their properties within certain limits and with certain rates. If, therefore, other species 'waiting in the wings' are better suited to the prevailing conditions, they will take over and out-compete the species present in the reservoir ecosystem (Jørgensen \& Nielsen.1994).

So far, ecological dynamic modeling has not looked closely at any other water related research fields because of its extremely complex mechanisms including a range of alternative disciplines. This kind of eco-model must be an important sub-model of ecohydrodynamics. 


\subsubsection{Data-driven Modelling}

Most environmental data have particular relationships between variables of data. Relationships can be derived between environmental variables that may have little or nothing to do with the physical principles of the underlying processes.

Many environmental data also exist as sequences of measurements over time or space. The time sequence is obvious in some data series, like weekly measurements on reservoir water quality at several sampling points. The influence of one variable on another can be spread over several time periods, for instance, instantaneous and/or lagged. A characteristic of such data can be that neighboring observations tend to be somewhat alike. High values are followed high values, and low values are followed by low values with an intermediate value or time lag. Because of these characteristics of environmental data, time series analysis with the computer software becomes more available. It is likely that applications to environmental forecasting will be on the increase.

As mentioned above most environmental data have a certain kinds of relationships each other. These do not necessarily imply that one variable causes the other, but that there are some significant associations among them, which are hard to express in mathematical equations. Here is one of the reasons that this study makes the case for the introduction of data-driven models to reservoir water quality modelling.

The basic data-driven model is to work with data on the boundaries of the domain where data are given, and to find a form of relationship(s) that best connects specific data sets (Price, 2002). Thus, it is computationally intensive as well. A data-driven model borrows techniques developed in such (overlapping) areas as statistics, computer science, and artificial intelligence (e.g. artificial neural networks and genetic algorithm), model trees (MTs) and soft computing, which mainly comprises fuzzy logic, artificial neural networks and probabilistic reasoning.

In recent years there has been a growing tendency to use data-driven model to complement or even replace deterministic models especially for forecasting. Although a simple example of data-driven model is a linear regression model, more complex models are highly non-linear and use sophisticated techniques. Such data-driven model has proved to be attractive in modelling algal species dynamics due to limitations in ecological knowledge for deductive modeling and limitations in data analysis for inductive modeling (Recknagel et al., 2002; Soyupak and Chen, 2004; Karimipour et al., 2005; Jung and Price, 2005).

Data-driven modelling approaches, which are the exploration and analysis by automatic or semiautomatic means of large quantities of data in order to discover meaningful patterns, relationships and rules, involve learning in a practical, not just a theoretical sense. 
The implemented data-driven model for this study is model trees (MTs) because of its more explicit and easy integration with each rule and linear regression. Existing MTs use a direct binary classification rule to construct their tree structure, and then each treestructured node associates leaves with multiple linear regression functions, calculating numeric values by the least square error (LSE) method. As an alternative, the clusteringclassification and the partial least square (PLS) method is applied for the tree generation and the linear regression at each leaf. A clustering-classification is more suitable for reflecting the association between the environmental data than binary classification, and partial least square (PLS) method is more effective than the LSE method in situations where the data is deficient.

\subsubsection{Decision Support System}

Environmental sciences comprise the scientific disciplines, or parts of them, that consider the physical, chemical and biological aspects of the environment (Allaby, 1996). A typical representative of environmental sciences is ecology, which studies the relationships between members of living communities and between those communities and their abiotic (non-living) environment with all of the (physically-based and datadriven) models mentioned above and using bio-assessment. Such a broad, complex and interdisciplinary field holds much potential for the application of knowledge discovery to the databases of hydroinformatics.

The basic categories of decision support systems can be distinguished according to knowledge inference methods as follows (Cordier, 2006):

- A model-driven decision support system emphasizes access to and the manipulation of a simulation model. This system uses data and parameters provided by experts to aid decision makers in analyzing a situation.

- A data-driven decision support system emphasizes access to and manipulation of an internal and, sometimes, external data warehouse.

- A knowledge-driven decision support system provides specialized problem solving expertise stored as facts, rules, procedures, or in similar structures.

- The new innovation of this study is to propose and to experiment with a decision support system such as shown in Figure 1.6 using interdisciplinary approaches: water quality assessment, eco-hydrodynamic modelling and data-driven modelling including the three categories above.

- The appropriate models can then be used to understand better the domain at hand or to predict the behavior of the studied communities and thus support decision making for environmental management. Although an ecological model (e.g. algal species composition in a reservoir) is not included here, it will be a crucial part of the hydroinformatics approach including cooperation with biologists. 


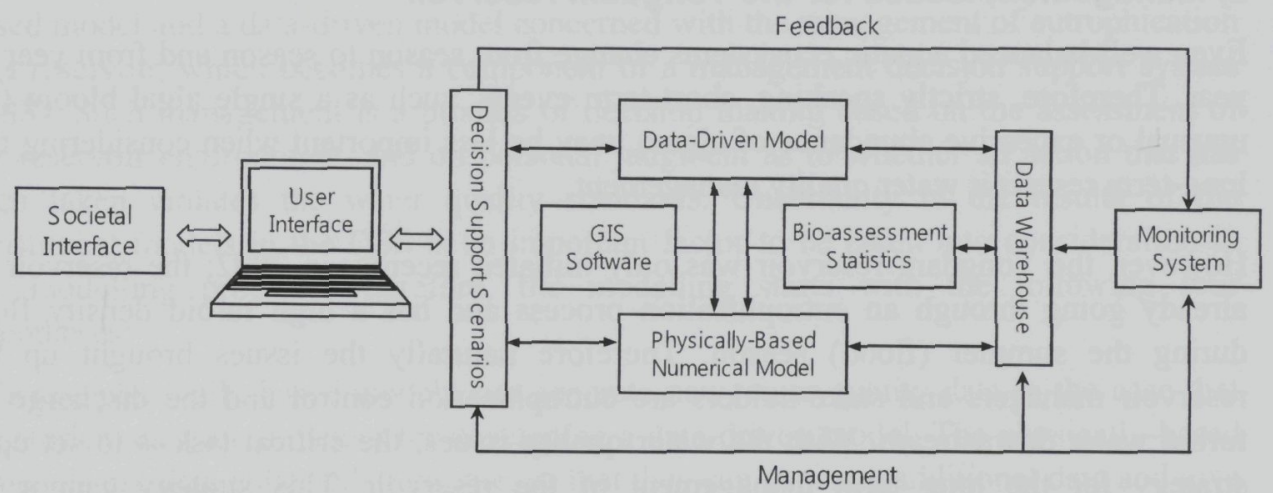

Figure 1.6. Decision support system considered for the reservoir water quality management

\subsection{Study Site}

\section{1) Location}

The Yongdam reservoir is formed as a result of the Yongdam impoundment upstream of Geum river, which has two reservoirs Daecheong and Yongdam reservoirs in its upper region. Figure 1.7 illustrates the Geum river basin located in the middle of South Korea, with a drainage area of $9,810 \mathrm{~km}^{2}$ with a mainstream length of $396 \mathrm{~km}$.

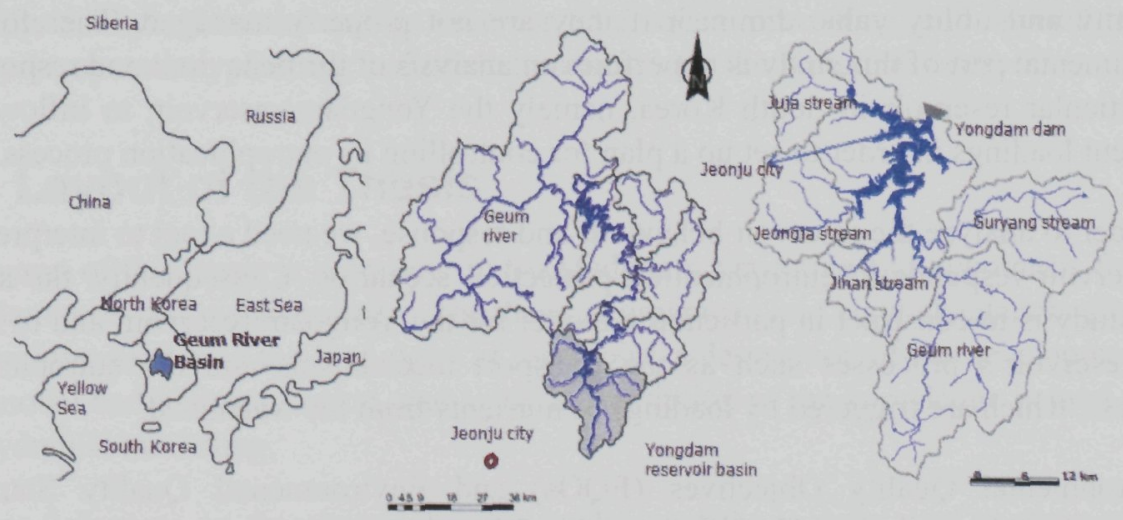

Figure 1.7. Location of the Yongdam reservoir on Geum river in South Korea.

The Yongdam reservoir was constructed for water supply to the nearby city of Jeonju $\left(700,000 \mathrm{~m}^{3} /\right.$ day with a planned increase to $1,050,000 \mathrm{~m}^{3} /$ day) for hydropower generation $(24,400 \mathrm{kw})$ and for flood control (volume of flood control: 137 million $\mathrm{m}^{3} /$ year). 


\section{2) Management Issues for the Yongdam reservoir}

Even well-balanced aquatic ecosystems change from season to season and from year to year. Therefore, strictly speaking, short-term events, such as a single algal bloom (an unusual or excessive abundance of algae), may be less important when considering the long-term reservoir water quality management.

However, the Yongdam reservoir was only initiated recently in 2002; the reservoir is already going through an eutrophication process and has a high turbid density flow during the summer (flood) season. Therefore naturally the issues brought up by reservoir managers and stake-holders are eutrophication control and the discharge of turbid water downstream. With the water quality issues, the critical task is to set up a strategy for the long term management of the reservoir. This strategy cannot be established without a proper analysis of the reservoir water, the nutrient transport mechanisms and the eutrophication processes. For this analysis, a simulation model for the transport and bio-chemical reactions is needed as a tool.

Thus the issues of the Yongdam reservoir can be summarized as: how to control the eutrophication processes; how to discharge interflow turbid water during the flood season, how to analyze the reservoir environment and how to correspondingly evaluate proposed water quality protection measures?

\subsection{Study Aim, Objective and Hypothesis}

It can be concluded that freshwater from reservoirs is a limited resource such that its quantity and utility value diminish if they are not properly managed. Therefore the fundamental part of this study is to perform an analysis of the behaviour and response of a particular reservoir in South Korea, namely the Yongdam reservoir, to inflows and nutrient loadings in order to set up a plan for controlling its eutrophication process.

In order to analyze the reservoir behaviour and response, we need a tool to interpret how a reservoir responds to eutrophication protection scenarios. Consequently, the aim of this study is to construct in particular a model for the Yongdam reservoir, and to assess the reservoir's processes such as the transport mechanisms and the eutrophication process, which are triggered by loadings of nutrients from the watershed.

Environmental Quality Objectives (EQOs) and Environmental Quality Standards (EQSs) in this study are identified by the following activities: data acquisition and analysis, model construction and instantiation, finally hybrid modelling. The model analysis was realised via two major modelling technologies, including two and three dimensional physically-based models, and data-driven models, which are based on a combination of statistics and artificial intelligent machine learning algorithms. Finally, a hybrid model uses the results of the physically-based modelling as input data for the data-driven modelling which is trained with measured data to get better results. 
This study is aimed at the construction of the hybrid model combining a physicallybased model and a data-driven model concerned with the management of eutrophication in a reservoir, which becomes a component of a management decision support system (DSS). Such management is a process of decision making based on the assessment of the reservoir environment, and on personal judgment as to whether an action that has been taken violates the water quality standards. Uncertainty in the results of the constituent models in the DSS is an important factor to be taken into consideration in the modelling process. Therefore, the modelling starts with the following four hypotheses:

- Firstly, physically-based models can generate new water quality data in the case that historical data are deficient for developing a data-driven model. The physically-based models use data collected at the site, so that they can generate additional data and give information on the reservoir response to nutrient loads for developing data-driven models and time series analysis of eutrophication processes.

- Secondly, data-driven models use bi-weekly observed data, which can deal with implicit and explicit knowledge about the reservoir performance, along with uncertainties only in the data. Data-driven models can be updated continuously and easily with data over time, and thereby increase their prediction level because ecoenvironmental data have reproducible cycles and relationships among attributes of data.

- Thirdly, the hybrid model developed in this study is a result of the interaction of a physically-based model with a data-driven model, which reduces uncertainties in the results of the physically-based model by substituting unknown parameters with information provided by the data-driven model.

- Finally, the hybrid model can be used for time series forecasting, and applied to the analysis, operation, planning and prediction works for the Yongdam reservoir.

\subsection{Layout of the Thesis}

The layout of this thesis is according to the study aim, objective and hypothesis for the Yongdam reservoir management. The thesis is organized into eight chapters beginning with an introduction to the limnology of reservoirs, a description of the development of each model and its instantiation, and lastly a description of the knowledge gained from eco-hydraulic modelling.

Chapter 2 focuses on the chemical water quality and the mainly algal ecology of the reservoir. This chapter may be read as a guide to the interpretation of the results of water quality modelling, including both physically-based and data-driven modelling as used in this study.

Chapter 3, 4 and 5 deal with the background of reservoir mixing, the functional equations and associated numerical modelling, and data-driven modelling involving machine learning techniques. These three chapters provide the backbone for the 
development of a tool for reservoir water quality management.

Chapter 6 describes the data acquisition and the primary analysis of the reservoir water quality data. The data include water quality parameters and algal growth potential test (AGPT) in the reservoir and at the five tributaries as well as the hydrological data. Firstly, the data acquired is used for an assessment of the Yongdam reservoir water quality and ecology and then for model building and application.

Chapter 7 deals with the instantiation of the models and the implementation of the methodologies described in chapters 3,4 and 5. The main contributions of this chapter are to the building and application of two and three dimensional physically-based models and data-driven models. A hybrid model combining a physically-based model and a data-driven model is implemented as a tool for decision making including forecasting and prediction. This chapter also deals with an analysis of the simulation results of the Yongdam reservoir. In this analysis, the most suitable decision support scheme for the Yongdam reservoir management is sought.

Chapter 8 deals with the conclusions of the whole study, not only concerning the Yongdam reservoir but also a new paradigm for the development of a hybrid model. Finally, recommendations are made for further study and the consequences for hydroinformatics. 


\section{Chapter 2}

\section{RESERVOIR ECOSYSTEMS}

\section{Summary}

This chapter describes the physical, abiotic and biotic structures and their influence on bio-chemical processes in reservoir ecosystems. These structures and processes in reservoir ecosystems are involved in eutrophication processes. Eutrophication is an ecological process driven by increasing nutrient availability which disrupts the natural equilibrium between biomass production and available nutrients due to a continuing process of nutrient accumulation in a reservoir. Therefore this chapter focuses on giving information about components and the production of reservoir ecosystems, realizing that there are many other particular characteristics that could be discussed; only the fundamental ecosystem concepts are touched on here.

\subsection{Physical Structure}

\subsubsection{Longitudinal Zonation}

Impoundment reservoirs tend to be long sinuous and dendritic in shape. Kimmel and Groeger (1984) developed a pictorial model to illustrate the expected changes in reservoir characteristics, from the inflowing river to the dam (see Figure 2.1).

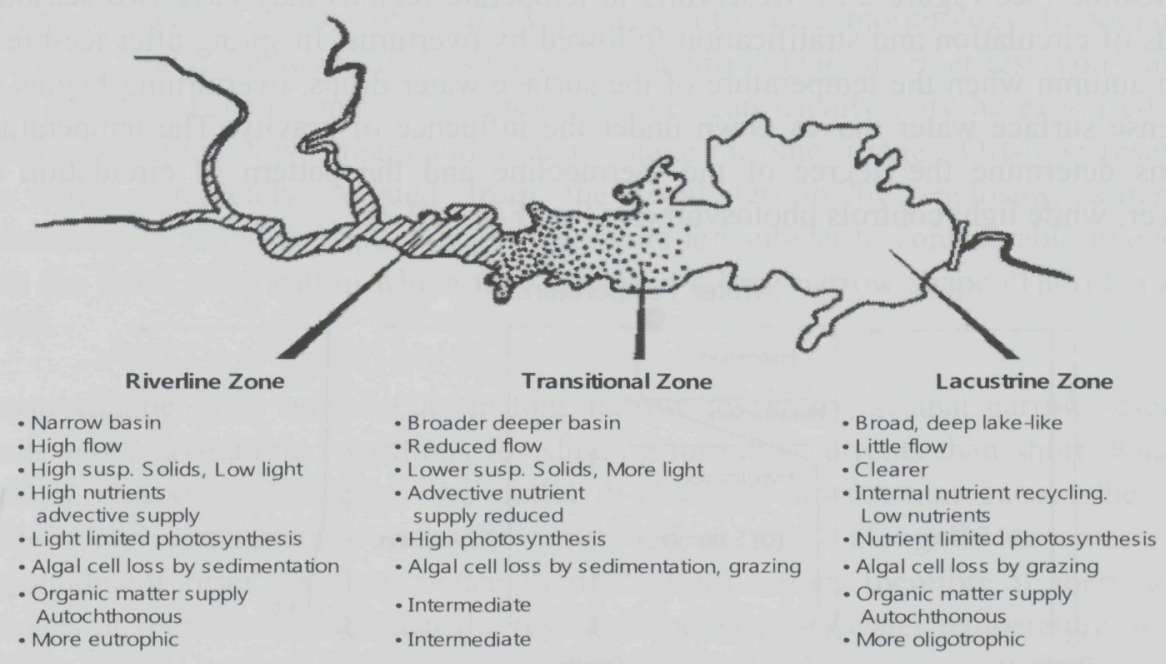

Figure 2.1. Longitudinal zonation in environmental factors which control primary productivity, phytoplankton biomass, and trophic state within reservoir basins. Changes in shading indicate decline in turbidity (Kimmel and Groeger, 1984). 
Many natural lakes have relatively homogeneous water quality, because water may enter from several smaller tributaries draining comparatively small sub-watersheds. In contrast, reservoirs have a distinct riverine zone which is dominated by flow and mixing, in which nutrient supplies are distributed by advective transport. This is followed by a transition zone where inflow velocity is slow, rapid sedimentation begins, and water clarity increases. The lacustrine (lake) zone near the dam is most like a lake, with thermal stratification and a higher probability of nutrients limiting algae growth. This zone often becomes a sink for nutrients originating from incoming rivers, and nutrients are supplied by internal recycling with dispersive transport. Algal cells are reduced by grazing predators and decomposed before reaching the bottom of the reservoir. Many reservoirs have such rapid and significant changes in water levels that the zones are changeable, and a littoral community of rooted aquatic plants is marginal or absent as a result. Important physical characteristics (or features) of reservoirs are the physical dimensions (length, depth and width), variations in temperature and the nature of the transport mechanisms. These characteristics lead to relatively small flow-through velocities and in deep reservoirs with cold winters and warm summers the development of significant vertical gradients in temperature and other water quality variables. The relationship between length, depth, area of water surface and volume is important (Thomann and Mueller, 1987), as are the currents responsible for the various transport mechanisms.

\subsubsection{Thermal Stratification}

The climatic regime affecting a freshwater ecosystem is a forcing factor for ecoenvironmental processes. One factor is water temperature, which may lead to thermal stratification (see Figure 2.2). Reservoirs in temperate regions may have two seasonal periods of circulation and stratification followed by overturns. In spring after ice-break and in autumn when the temperature of the surface water drops, overturning begins as the dense surface water moves down under the influence of gravity. The temperature patterns determine the degree of the thermocline and the pattern of circulation or turnover; while light controls photosynthesis (see Figure 2.2).

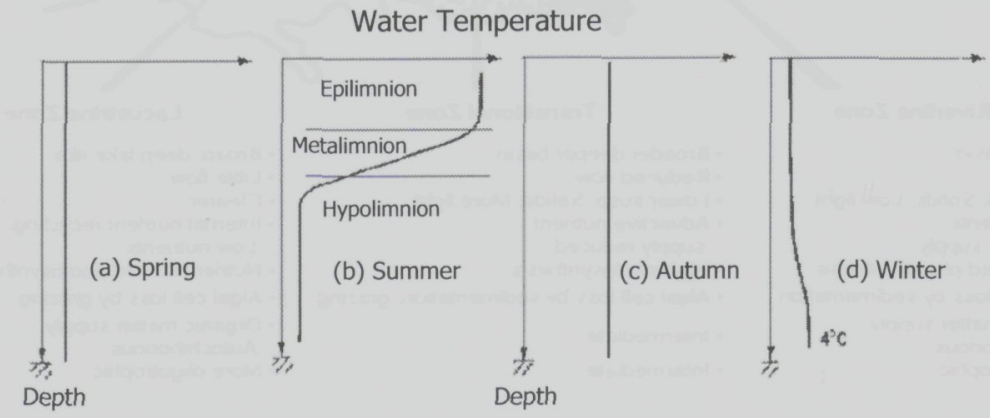

Figure 2.2. Possible variation of water temperature with depth throughout the year, for a reservoir in a temperature region. 
This process is aided by wind or currents that oscillate the water as a form of seiche. The seiches induced by flow do not change with time, but vary in depth as ones move into or against the flow.

Stratification in the water column due to density differences seriously inhibits vertical circulation. Whereas many processes are responsible for mixing in reservoirs, e.g. oxygen exchange between the surface and bottom layers, vertical mixing in case of thermal stratification is mainly limited to convective currents generated in the case of a locally unstable stratification, and intrusion flows in stably stratified environments. While stratification normally reduces vertical exchange, it can drive horizontal exchange by forming a strong vertical structure.

Other causes of stratification are due to variations in dissolved solids and suspended particulates; however these are generally negligible in the bulk fluid flows of inland reservoirs (Socolofsky and Jirka, 2005). In fact, this temperature (density) difference between the layers also affects the physical process of reservoirs because it may reduce the effective gravitational acceleration in the settling process of particles by a factor 100 to 1,000 (Basson, 1998). Thus, stratification is dominantly dependent on the variation in temperature, which in turn is a function of the overall energy balance and the internal mixing processes of a reservoir. When the temperature of the surface water reduces, the stratification is unstable and the reservoir water overturns, lifting up anoxic bottom water. The reservoir eco-hydrodynamics are described in more detail in Chapter 3.

\subsubsection{Reservoir Morphology}

The two main types of artificially created water bodies are impoundment reservoirs and off-river reservoirs. These are usually very different in their morphology. Impoundment reservoirs tend to be larger, more sinuous and dendritic than off-river reservoirs and the main control of their contained volume of water can usually only be exercised at an outlet discharging downstream and/or at an intake for other usages. Off-river reservoirs are often completely isolated from the local terrain by enclosing waterproof embankments, whereas impoundment reservoirs are subject to considerable interaction with the flood plain within which they can form a long narrow shape (Thornton et al., 1996).

Linear channel flow only occurs in long-narrow reservoirs, so that narrow reservoirs create more favourable conditions for sluicing turbidity currents than short, wide and shallow reservoirs. However, the outlet discharging downstream causes the turbid inflows and contaminants from the watershed to travel along the reservoir. Most impoundment reservoirs experience thermal stratification; therefore if there are no services or regulations such that the reservoir increases its water temperature, it is the surface water, having a higher temperature than the lower layer that is normally discharged downstream. In this case, high turbid and dense water flowing at the thermal interface stays longer in the reservoir. This factor, together with an outlet positioned to 
one side (mainly dam structure), makes it difficult to control reservoir water quality in a long impoundment reservoir.

\subsubsection{Outlet Location}

The main control of the reservoirs' contained volume of water can usually only be exercised at an outlet discharging downstream and/or at an intake for other usages (Thornton et al., 1992). Locations of outlet structures are positioned according to specific purposes of the reservoir. These positions of outlet and operational releases affect flow and stratification conditions within the reservoir (Straškraba and Tundisi, 1999). Most large dams in Korea have deep cold water discharge facilities, and this cold discharged water is stored in a second reservoir created by a regulation dam, which is constructed just downstream of the main dam in order to raise the temperature of the discharged water. Since the Yongdam reservoir has, however, a surface water withdrawal facility without a second reservoir, it is important to know what the differences are between deep and surface water discharges, for water quality management in the reservoir.

\subsection{Abiotic and Biotic Structure}

\subsubsection{Introduction}

An ecosystem can be defined as a structural and functional unit of the biosphere or a part of nature consisting of a community of living beings and the associated physical environment, such that both interact and exchange materials between them: ecosystems can be regarded as self - regulating and self - sustaining units (Odum, 1971; Voronov et al., 2002: Zhuravlev and Avetisov, 2006). The community of living organism are involved in dynamic biological, chemical and physical interactions between themselves and with the nonliving components. This community consists of two sets of components: abiotic and biotic (Odum, 1971). Abiotic components consist of the inorganic and organic substances that are subject to the climatic regime in the freshwater ecosystem. These abiotic components include nutrients that are made available in a given environment for the mass production of biotic components.

\subsubsection{Abiotic Structure}

\section{1) Inorganic Substances}

Inorganic substances in surface waters are mainly characterized by their bio-available ionic composition, and are dominated by major cations and anions. The prevailing components in open water bodies are (Sverdrup et al., 1942; Odum, 1971):

$$
\text { Cations: } \mathrm{Ca}^{2^{+}}>\mathrm{Mg}^{2+}>\mathrm{Na}^{+}>\mathrm{K}^{+}
$$




$$
\text { Anions: } \mathrm{HCO}_{3}{ }^{-}>\mathrm{CO}_{3}{ }^{2-}>\mathrm{SO}_{4}{ }^{2-}>\mathrm{Cl}^{-}
$$

In comparison, the amounts of nitrogen and phosphorus available as nutrients are very small. However, they are important for the production of biotic components in water bodies. The small natural supply of phosphorus is commonly the limiting factor for the production of biotic components. These nutrients as well as numerous trace elements are essential for biological development but they do not contribute substantially to the change in the total balance between cations and anions (Overbeck, 1988). For example, silica is essential for diatoms, whereas iron and manganese, which are important for metabolic processes, are essential for all species of algae. Besides these nutrients, calcium, magnesium, sodium, potassium, iodine and arsenic are present in all species, but it is not well known whether they are all essential.

\section{a) Phosphorus}

Living organisms require phosphorus for energy transfer within the cell, for several enzyme systems, and as constituents of DNA and RNA (Ishige et al., 2003). The kinetics and chemical reactions of phosphorus in the aquatic environment are not well defined. Thus, phosphorus in freshwater is often simply classified in terms of a few forms: soluble reactive phosphorus (SRP), soluble un-reactive phosphorus, particulate phosphorus and total phosphorus. Soluble reactive phosphorus, that is, dissolved inorganic phosphate (commonly taken to be orthophosphate $\left(\mathrm{PO}_{4}{ }^{3-}\right.$ and related forms; see here under) that passes a $0.45 \mu \mathrm{m}$ membrane filter) is known as being available for biota. However, some condensed phosphate forms, such as those found in detergents, are inorganic but not available for plant uptake.

Soluble un-reactive phosphorus, like that dissolved in organically bound phosphates excreted by organisms and as colloidal phosphorus (particulate phosphate) compounds such as apatite and organic particulate phosphorus included in living and dead plankton or detritus, can be available to biota through the action of phosphates, i.e. as enzymes emitted by microorganisms (Fitzgerald and Nelson, 1966; Wynne and Berman, 1980; Wetzel, 1981). Particulate phosphorus often constitutes the largest portion of total phosphorus. The fraction of dissolved orthophosphate can be quite small and often has an extremely short turnover time (often minutes). The occurrence of different phosphorus forms is dependent on $\mathrm{pH}$ (Zimmerman, 1980).

The equilibrium of the different forms of inorganic phosphate at different $\mathrm{pH}$ values in water is shown in Figure 2.3. Phosphoric acid $\left(\mathrm{H}_{3} \mathrm{PO}_{4}\right)$ dissociates into several hydrogen phosphate ions, depending on $\mathrm{pH}$. The soluble inorganic phosphate forms $\mathrm{H}_{3} \mathrm{PO}_{4}$, $\mathrm{H}_{2} \mathrm{PO}_{4}{ }^{-}, \mathrm{HPO}_{4}{ }^{2-}$ and $\mathrm{PO}_{4}{ }^{3-}$, known as soluble reactive phosphorus, are all readily available to biota. These solid inorganic phosphates may be available to the cells, if not immediately, then at some point in time (Giammatteo et al., 1980). Plants store phosphate in excess of their immediate needs, utilizing the excess under conditions of phosphorus deficiency at a later time (Vallentyne, 1974). It is recommended that phosphate concentration is expressed as phosphorus, and without indicating charges; i.e., $\mathrm{mg}^{-1}$

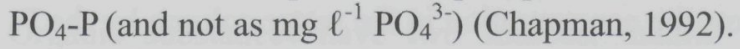




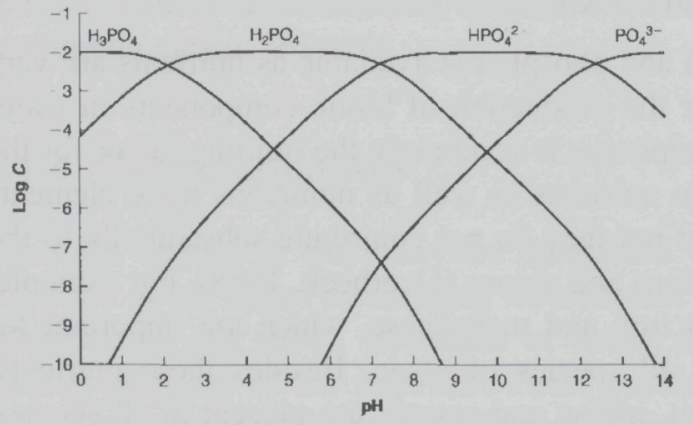

Figure 2.3. Distribution of phosphate species as a function of $\mathrm{pH}$ for a system containing $10^{-2} \mathrm{M}$ total orthophosphate (Benjamin, 2002).

\section{b) Phosphorous Cycle}

During the stratification in a eutrophic reservoir, the deeper anoxic layer (hypolimnion) has a steep increase of dissolved, mostly inorganic, phosphorus. The low exchange rate between the epilimnetic and hypolimnetic waters causes accumulation of phosphorus. Therefore, it may not be immediately available for epilimnetic primary production. Figure 2.4 portrays the transport and conversion of phosphate in a reservoir.

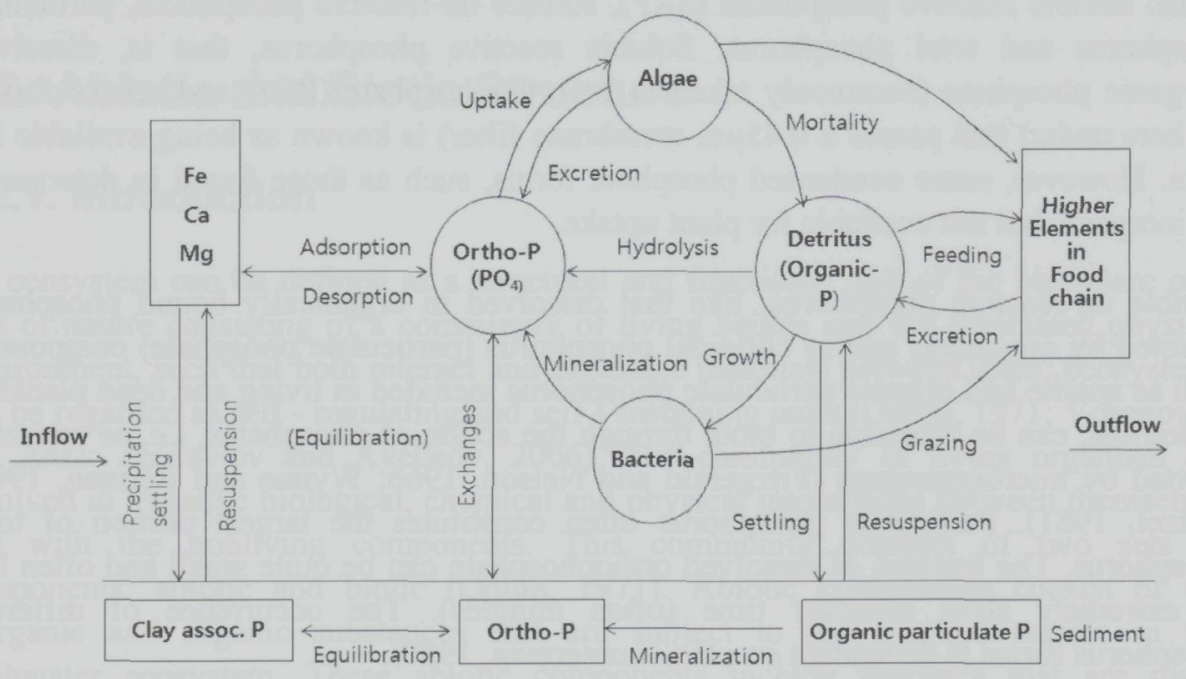

Figure 2.4. Transport and conversion of phosphate in a lake.

In the water column, all phosphorus forms are transformed to more bio-available forms at various rates dependent on microbial actions and environmental conditions. In reservoirs, because the residence times are sufficiently long for all phosphorus formation kinetics, total phosphorus is generally considered to be an adequate estimate of bio-available phosphorus. The bio-available forms are apparently triggered by photosynthetically induced increases in $\mathrm{pH}$ and redox conditions in the water column (see Figure 2.3).

In the sediment layer, phosphorus affects the concentration in the water column by the $\mathrm{P}$ release from the sediment (Kelderman, 1985; Nürnberg, 1988; Sas, 1989) and dredging 
of the top-P-rich sediment layers is often considered for reservoir restoration (Kleeberg and Kozerski, 1997). Thus, the cycle of phosphorus in a reservoir is primarily regulated by five factors: the inflow from the watershed, the biological uptake, the precipitation reactions with certain positively charged ions, a two-way flow between sediments and the water column, and the outflow to other waters. Research about the complex phosphorus chemistry in aquatic systems is beyond the scope of this study, and is therefore not discussed in more detail here.

\section{c) Nitrogen}

Nitrogen is a highly important nutrient because it is a necessary element in the structure of proteins. Chlorophyll, RNA, DNA, several coenzymes, and some vitamins also contain nitrogen. Thus nitrogen is essential for such important functions as photosynthesis, respiration, protein synthesis, formation of genes and growth. Dissolved nitrogen compounds include ammonia nitrogen $\left(\mathrm{NH}_{3}-\mathrm{N}\right.$ or $\left.\mathrm{NH}_{4}{ }^{+}-\mathrm{N}\right)$, nitrite $\left(\mathrm{NO}_{2}{ }^{-}\right)$, nitrate $\left(\mathrm{NO}_{3}{ }^{-}\right)$, dissolved molecular nitrogen and a high diversity of organic compounds (e.g. amino acids, proteins, nucleotides, and refractory humic compounds with low nitrogen contents). Oxygen has a decisive influence on the whole cycle, determining rates of nitrification $\left(\mathrm{NH}_{4}{ }^{+} \rightarrow \mathrm{NO}_{2}^{-} \rightarrow \mathrm{NO}_{3}^{-}{ }^{-}\right)$. Denitrification $\left(\mathrm{NO}_{3}{ }^{-} \rightarrow \mathrm{NO}_{2}^{-} \rightarrow \mathrm{N}_{2}\right.$ ) occurs only under anoxic or low oxygen conditions. Ammonia is nitrified largely by Nitrosomonas to nitrite. Oxidation of nitrite proceeds further to nitrate by Nitrobacter (Gode and Overbeck, 1972; Witzel and Overbeck, 1979).

In anoxic hypolimnetic waters, ammonia is accumulated due to decomposition of organic nitrogen forms and release of reduced nitrogen compounds from the sediment. In well-oxygenated waters, the concentrations of ammonia are generally low, and will depend on the actual state of metabolism of the aquatic ecosystem. Thus, after a breakdown of phytoplankton populations, higher amounts of ammonia may be present also in the aerated epilimnion, before nitrification of the reduced nitrogen compounds takes place. It is very common for the development of a "nitric plate" to occur in the metalimnion due to nitrification or denitrification of the metabolically extremely active bacterial populations at this depth in the reservoir (Jørgensen and Vollenweider, 1988). The exact mechanism effecting ammonium uptake by phytoplankton is uncertain due to the coexistence in solution of $\mathrm{NH}_{3}$ and $\mathrm{NH}_{4}{ }^{+}$(Raven, 1984). Normally $\mathrm{NH}_{4}{ }^{+}$constitutes over $90 \%$ of total ammonia, but $\mathrm{NH}_{3}$ will be the main form when external $\mathrm{pH}$ is high. Nitrate $\left(\mathrm{NO}_{3}{ }^{-}\right)$being the most oxidized form of $\mathrm{N}$ is taken up by algae under aerobic conditions and is then reduced by assimilation processes to the amine form which can be used in metabolic processes (Valiela, 1984). Nitrate is not as easily assimilated by algae as ammonium. Nitrite $\left(\mathrm{NO}_{2}{ }^{-}\right)$is present in much smaller concentrations in freshwater, and is therefore less likely to be assimilated by algae than $\mathrm{NO}_{3}{ }^{-}\left(\mathrm{D}^{\prime}\right.$ 'Elia and DeBoer, 1978).

\section{2) Organic Substances}

Organic substances have two sources: natural and anthropogenic. Natural organic 
compounds have a major controlling effect on the hydro-chemical and biochemical processes in a water body (Chapman and Kimstach, 1992). Dissolved carbohydrates, proteins, humic substances, pigments and vitamins comprise the major organic components of water. They are playing an important role in the aquatic ecosystem as extra-cellular dissolved organic matter (DOM), released by cell autolysis or excretion (Overbeck, 1988).

The anthropogenic organic compounds such as phenols and benzoic acids are major components in urban rainwater, in contrast to rural rainwater. Accordingly, anthropogenic organic compounds in reservoirs that are mainly located in the mountain area are minor constituents of the reservoir water (Kawamura and Kaplan, 1986). Anthropogenic organic compounds to reservoir water depend on the inflow water quality affected by atmospheric conditions (air quality, wind direction, precipitation rate, etc.).

\subsubsection{Biotic Structure}

In a food-chain or rather a set of interconnected food-chains (food-web), energy and materials circulate within an ecosystem. The food-web of reservoirs reflects the changes in the catchment and hydrological variations, and an ageing of the reservoir ecosystem might affect the biomass even several decades after filling (Straškrábová et al., 2005).

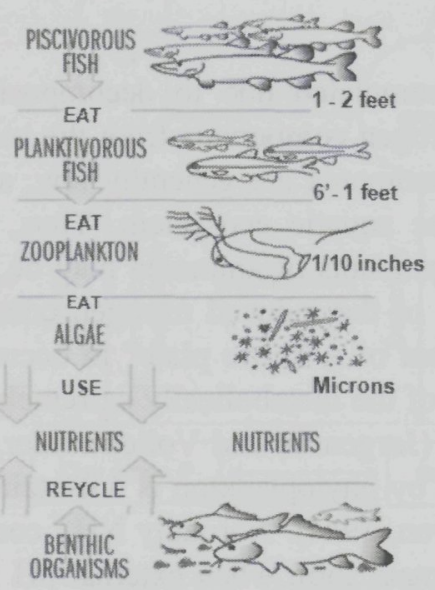

(a) General food-web in aquatic environment

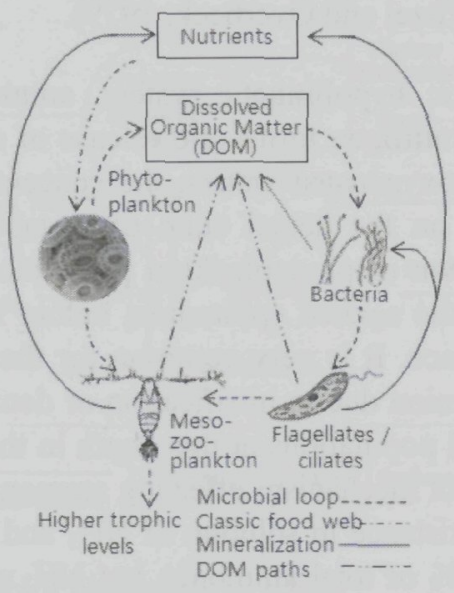

(b) Microbial loop in the food-web

Figure 2.5. A general food-web in reservoir (a), and Microbial loop inserted into the food-web(b) ( Source: Torréton, 2009).

The food-web in a reservoir can be divided into producers, macroconsumers, and microconsumers as shown in Figure 2.5 (a). Figure 2.5 (b) shows a microbial loop inserted into the food-web. Producers, autotrophic phytoplankton (algae) and higher plants (macrophytes) produce biomass from inorganic substances (nutrients) (Overbeck, 1988). Microconsumers, bacteria and fungi are responsible for degradation of the 
particulate and dissolved organic substrate. Macroconsumers, zooplankton and fish feed on other organisms or particulate organic matter (detritus).

A metabolic coupling of autotrophic and heterotrophic processes could be supposed from the distribution pattern of phytoplankton and bacteria (see Figure 2.5 (b)). Heterotrophic bacteria obtain their energy requirements via the oxidation of organic matter. Thus bacteria typically control microbiological processes of the nitrogen cycle in reservoirs: nitrification, denitrification, fixation of molecular nitrogen, and nitrate ammonification.

In the vertical profile of stratified eutrophic reservoirs both phytoplankton and bacteria have the highest concentration at the same depth. It is essential for the understanding of the food web that the correspondence between algae and bacteria is not only quantitative, but also qualitative (Overbeck, 1988).

\subsection{Eutrophication}

\subsubsection{Assimilation and Limiting Nutrient}

Eutrophication is an ecological process driven by increasing nutrient availability which disrupts the natural equilibrium between biomass production of phytoplankton (algae) and available nutrients due to a continuing process of nutrient accumulation in a reservoir. The algal concentration may be used as an indicator for eutrophication control, for example, total phosphorus emission control. These problems are major issues for reservoir water quality management.

All microorganisms require certain chemical elements for life. In algal cells these elements are chemically combined in an organism's body to form complex molecules and allow the conversion of substrate to energy (respiration), or to build organic molecules using energy from sunlight (photosynthesis). Cell material is the end product of this assimilation. Hence, a chemical analysis of the cell material gives an indication of what elements are required to build the cell. Numerous experiments in the laboratory and in the field have demonstrated the major role of phosphorus, nitrogen, and in some cases, silicon, influencing the dynamics of algal populations including algal concentrations and species composition (Forsberg et al., 1978).

The maximum biomass of algae is determined by the nutrient supply and light availability. When a nutrient which is essential for growth of aquatic plants becomes unavailable (because its pool is exhausted or it is locked up in an unavailable form) the algal growth becomes limited by that nutrient (Robertson, 1997). The concept of limiting nutrients has its basis in the photosynthesis reaction. Although the exact stoichiometry of this reaction is still debated, the chemical needs of phytoplankton photosynthesis and respiration can be presented conceptually (Stumm and Morgan, 1970) as follows: 


$$
\begin{aligned}
\left(\mathrm{CO}_{2}+\mathrm{N}+\mathrm{P}+\mathrm{H}_{2} \mathrm{O}+\mathrm{H}^{+}+(\text {trace element, sunlight })\right)_{n} \\
\stackrel{\text { Respiration }}{\stackrel{\text { Photosynthesis }}{\rightleftarrows}} \mathrm{C}_{106} \mathrm{H}_{263} \mathrm{O}_{110} \mathrm{~N}_{16} \mathrm{P} \text { (algal biomass) }+\mathrm{H}_{2} \mathrm{O}+\mathrm{O}_{2}
\end{aligned}
$$

The ratio of the nutrients taken up to that used by the organism reflects the composition of the elements in the cellular material of the organism. The chemical composition of a living cell, however, is variable. According to above equation, the average atomic ratio of these three elements C:N:P in the plankton (algae) is 106:16:1 (Redfield, 1934; Falkoeski, 2000). This ratio has become a widely cited reference value for assessing limiting nutrient in water bodies (Uhlmann, 1982). Carbon normally becomes an algal growth-limiting nutrient only when the water is saturated with both phosphorus and nitrogen, when light availability and temperature are high, and when the transport of carbon dioxide from the atmosphere to the water column is slow. Even in these unusual circumstances, carbon limitation usually only affects the types, rather than the quantity, of algae occurring in a water body (James and Lee, 1974).

$\mathrm{N}$ and $\mathrm{P}$ are taken up by phytoplankton at a mass ratio of, on average, 7.2:1 in correspondence with above $16 \mathrm{~N}: 1 \mathrm{P}$ atomic ratio reference value. If the available amounts of $\mathrm{N}$ and $\mathrm{P}$ differ widely from this ratio, a limitation in the production results. The critical N:P mass ratio is often taken as 10:1 (Forsberg et al., 1978). However, precautions should be taken in the practical use of the limiting nutrient concept (Lee et al., 2000; PHOSLOCK, 2008). First, strictly speaking, the concept applies only under steady-state conditions. Another consideration is that a water body normally contains a number of different algal species, each with relatively specific nutrient requirements (Glibert, 2007).

Different algal species can assimilate nutrients in different quantities and at different rates (Tilman, 1982). How well one species can compete with another for available nutrients depends on nutrient uptake and assimilation rates. This is the basis of the concept of resource competition by algal populations (Hutchinson, 1961; Kilham, 1978; Tilman, 1982). Tilman (1982) provided support for this concept with the observation that diatoms appear to be superior competitors for phosphorus, but inferior competitors for nitrogen and silica. Thus, diatoms grow better under low phosphorus levels than other algal species. Lang and Brown (1981) reported that some blue-green algae were more efficient in phosphorus uptake under phosphorus-limited conditions than some green algae.

The relative proportions of aquatic plant nutrients in reservoir can also change seasonally and year-to-year. Thus, one nutrient may limit algal biomass levels at one time, while another nutrient may be limiting at another time. Practical use of the limiting nutrient concept, however, assumes that a single nutrient required by algae will be the limiting factor, at least during the period considered.

Taking into account above considerations, it is generally agreed that nitrogen is the limiting nutrient for algal growth in coastal marine ecosystems, while in freshwater systems it is usually phosphorus (Correll, 1998; Howarth and Marino, 2006). In terms 
of algal growth, phosphorus in freshwater bodies in Korea has been found to be the more limiting nutrient than nitrogen (Shin, 2003a, 2003b; Kim et al., 2007). Therefore, limiting the input of these nutrients, and particularly phosphorus, is a primary option for controlling eutrophication.

\subsubsection{Environmental Factors}

Compared with knowledge on the physical structure, reservoirs are less understood with respect to nutrient limitation for algal growth, except for assuming similar conditions as in lakes, which are primarily phosphorus-limited water bodies (Correll, 1998). The phytoplankton biomass in the water body appears to be proportional to the nutrient load, at least up to a certain level. Beyond this level, the phytoplankton exhibits a relatively constant value, showing no further increases in algal growth, regardless of further increases in the nutrient supply (Cowen and Lee, 1976; Sridharan and Lee, 1977; Lee et al., 1980). This means that factors other than nutrient availability can also limit the algal growth.

These factors may be highly interdependent physical transport mechanisms such as the amount of light available, water temperature, residence time, hydraulic mixing and also characteristics of the contributing watersheds including their morphology, soil type and land use (Ford, 1990; Kennedy and Walker, 1990; Ambrosetti et al., 2003; Burge and Breen, 2006). For this reason, the potential impact of external nutrient loads on the reservoirs' ecosystems cannot be adequately determined on the basis of physical and chemical features alone (López and Dávalos-Lind, 1998). An algal growth potential test (AGPT) in this study has been used to analyse the maximum algal growth that can be produced in water bodies regardless of unknown factors inhibiting algal growth and limiting nutrient of the thermally stratified Yongdam reservoir (see Chapter 6.3).

\subsubsection{Algae}

Algae are a very diverse group of organisms. Over the year, algal species dominance in lakes changes in a yearly cycle, known as algal succession (Kortmann and Henry, 1990). This natural succession of algae occurs in response to changes in season, temperature, wind, precipitation patterns, and nutrient cycles (Moore and Thornton, 1988) and in line with the nutrient uptake capabilities and requirements for surrounding conditions for the different species (Shapiro, 1970; Silvey et al., 1972).

Algal populations are abundant in spring and early summer when water temperature and nutrients concentrations are high and few organisms are present to feed on the algae. Toward the end of this stage, a phenomenon, commonly known as the clear water phase, occurs in many lakes. Spring algal populations are usually composed of small, highly edible species. As this phase approaches, zooplankton populations increase dramatically. This zooplankton consumes algae rapidly, causing algal populations to crash, resulting in very clear water for a few weeks (Addy and Green, 1996). These small, edible algal populations are gradually replaced by larger, colonial, non-edible species that are often 
covered by gelatinous sheaths. Because available nutrient concentrations are often limited in the summer, the total concentration of algae in summer can be less than in spring; another factor may be dilution by floods. In late summer and fall, nutrients stored at the bottom of the lake become mixed through the turnover generating a fresh supply of nutrients. This allows algal populations to flourish again and late season algal blooms to develop. During winter months, algae are able to survive, but usually at low concentrations due to colder water temperatures. Sometimes species composition tells more about the structural condition of a lake or a reservoir than water quality. An example is the presence of blue-green algae which cause problems of odour and taste (Gerber and Lechevalia, 1965; Kim et al., 1997); this is often a much greater environmental problem than a low transparency or high nutrient concentrations.

Algal blooms contribute significantly to the total organic material (carbon) content in the water through metabolic processes within algal cells and their autolysis. Increased organic matter induced by biomass production, requires a larger disinfectant dose in the drinking water treatment process. Also humic and fulvic acids, which are organic materials that can be produced by algal blooms, act as precursor substances in the formation of trihalomethanes such as chloroform $\left(\mathrm{CHCl}_{3}\right)$, as well as other halogenated health hazardous by-products (bromoform $\mathrm{CHBr}_{3}$, bromodichloromethane $\mathrm{CHBrCl}_{2}$ ) (Moore and Thornton, 1988). These by-products have been declared as probable human carcinogens and there is growing apprehension that the number of regulated compounds reflects the current, limited epidemiological knowledge rather than the actual potential health risk (IARC, 1992; US EPA, 1994).

The algae that pass through the settling basin and are trapped in-between the sand grains in a filter bed of a treatment works can cause gradual or rapid loss of head. Although effective coagulation and sedimentation can remove up to 90 or $95 \%$ of the incoming algae, the remainder may be sufficient to shorten filter run times significantly. Diatoms can further hasten filter clogging due to their rigid cell walls. One of the green algae, Palmella, forms copious mucilaginous material around its cell and literally gums up the filter bed (Montgomery, 1985). 


\section{Chapter 3}

\section{PHYSICAL PROCESSES OF RESERVOIRS}

\section{Summary}

This chapter describes the physical processes concerning density currents and mixing in a reservoir, and the algorithmic expressions for these processes. Flows are characterised according to density differences (between inflows and the receiving water) and eddy sizes that have three turbulent length scales: the integral scale, the Taylor scale, and the Kolmogorov scale. Turbulence can be regarded as instability in laminar flow that occurs at high Reynolds number. Such instabilities originate from interactions between nonlinear inertial terms and the viscous forces. These interactions lead to vortex stretching of rotational, fully time-dependent and three-dimensional flow. The Navier-Stokes equation can be used to describe and interpret these flows from the basic principles of conservation of mass, momentum, and energy for both laminar and turbulent flows included at the scale of interest. DNS, LES and RANS are solution methods of the equation. In spite of recent advances in computing, it is difficult to calculate a general turbulent fluid motion using these methods because of the high simulation cost. As a result, practical applications require other solution techniques to reduce the application of the basic equations to a more manageable form. This chapter mainly focuses on these approaches, including the Reynolds-Averaged Navier-Stokes simulation based on the statistically averaged two-dimensional eddy viscous equations, and includes a brief turbidity current.

\subsection{Mixing Processes in a Reservoir}

\subsubsection{Major Transport Mechanisms}

Reservoir physical processes include many transport mechanisms (see Figure 3.1) or currents (Fischer et al., 1979; Ford, 1990; Imboden and Wüest, 1995; Chapra, 1997). All of these transport mechanisms occur in reservoirs at one time or another. It is also probable that different transport mechanisms will dominate at different locations within a reservoir at a given time. The specific types of transport mechanisms that occur depend on the type, magnitude, duration, and variation of the forcing variables (i.e., energy sources).

The movement and mixing of dissolved and particulate matter within a water body also results from a number of complex, interdependent, physical transport mechanisms (Ford, 1990). Because these mechanisms influence the environment (i.e., temperature, light, and chemical regimes) in which aquatic organisms exist, an understanding of reservoir density and turbidity currents which are important transport mechanisms, is an essential 
prerequisite for the study of water quality management in reservoirs. The major transport mechanisms in reservoirs shown in Figure 3.1 include the following.

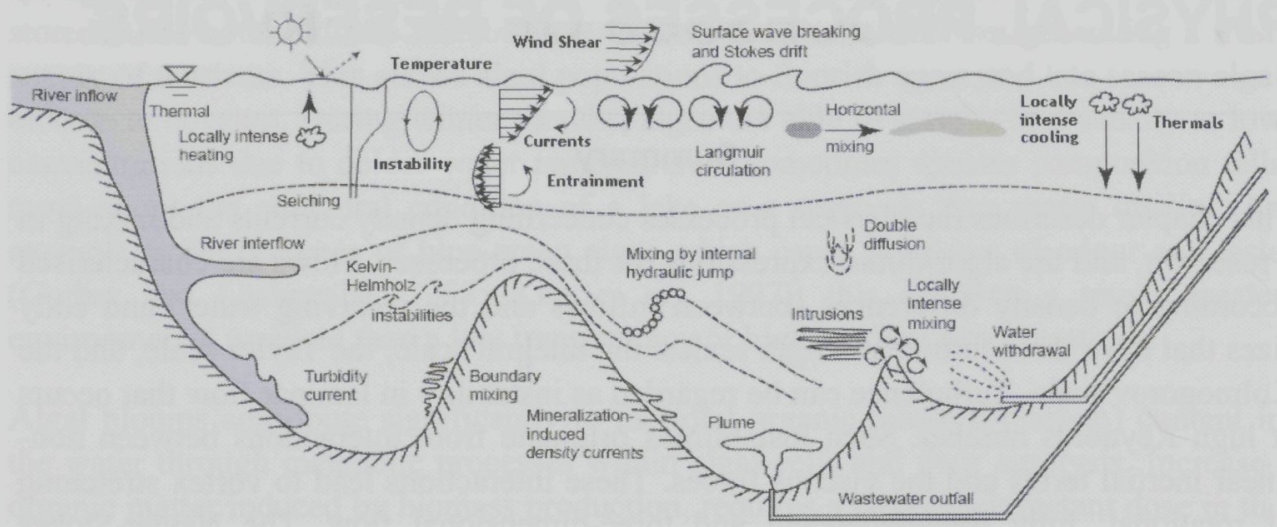

Figure 3.1. Schematic of the mixing processes in a lake (Source: Imboden and Wüest, 1995).

- Advection is transport by an imposed current system, which may be caused by river inflows, outflows, and wind shear at the air-water interface. Advection is unidirectional and does not change the identity of the substance being transported, but moves matter from one position in space to another.

- Convection is vertical transport induced by density instabilities due to the sinking of denser surface water due to cooling.

- Turbulence is generated by wind, inflows, outflows, convection, shear stresses at boundaries, etc. It is sometimes described as a family of eddies (e.g., an eddy is the swirling of a fluid and the reverse current created when the fluid flows past an obstacle) that range in size from the scale of the physical boundaries of the flow-field down to molecular motion. Therefore turbulence is irregular, diffusive (produce mixing), rotational, time varying, and dissipative (decays rapidly without a continual source of energy).

- Diffusion is a mechanism where differences in mean concentrations are always reduced without any overall transport of a fluid taking place. Molecular diffusion resulting from the random Brownian motion of water molecules is an intrinsic property of the fluid and varies from solute to solute. In contrast, turbulent diffusion is the random scattering of particles by turbulent motion with a larger eddy diffusion coefficient than for molecular diffusion. Turbulent diffusion is not a property of the fluid but rather a function of the flow regime.

- Shear is the advection of a fluid at different speeds at different positions. It can be generated at the air-water interface by wind, along the bottom boundary, by an inflow current, and internally by density currents.

- Dispersion is the combination of the effects of shear and diffusion. Since there is usually little information on the velocity distribution in reservoirs, it is difficult to 
separate dispersion from diffusion. In general, dispersion should dominate in the headwater regions of a reservoir, where the riverine velocities are still large, and diffusion should dominate in the main pool of the reservoir, where current velocities are small.

- Entrainment is an advective-type transport mechanism where the thickness of the stirred layer grows by entraining (trapping) fluid from the unstirred layer. The interface between the stirred and unstirred layers, therefore, advances into the unstirred layer. In reservoirs, metalimnetic water is entrained into the epilimnion by wind-generated turbulence or convection, and the depth of the epilimnion grows. Ambient reservoir water can be entrained into an inflow density current by turbulence generated at the sediment-water interface. The thickness of the inflow density will therefore increase. In contrast to diffusion, entrainment sharpens gradients.

- Mixing is any mechanism or process that causes a parcel of water to blend with or be diluted by another. Mixing includes diffusion, shear, dispersion, and entrainment.

- Settling is the sinking of particles with densities different from (and greater than) the surrounding fluid.

- Stokes drift is the small time-averaged drift velocity of suspended non-diffusing particles in a fluid due to the presence of a wave.

- Langmuir circulation was first recognized in 1938 by Irving Langmuir, who observed patterns of debris on the surface of the sea in the North Atlantic. What happens is that in some instances the wind causes the formation of cells of rotating water that parallel the surface.

- Kelvin-Helmhotz instabilities is a hydrodynamic instability due to the Earth's rotation (Coriolis force) which is everywhere exactly balanced by the components of gravity along a wave crest that slopes downward from right to left in the direction of progress (Northern Hemisphere), so that right hand shore line coincides with maximum wave amplitude.

- A seiche is a Swiss French dialect word that means 'to sway back and forth', and a seiche wave was originally identified through oscillations observed in alpine lakes. These oscillations are caused by resonances in a body of water that has been disturbed by one or more of a number of factors, most often wind or seismic activity.

Thermal mixing and advection-dispersion (AD) are the most important aspects of reservoir physical processes. Temperature is usually the only parameter that can be used in practice for the calibration of hydraulic models for reservoirs. However, since temperature is affected by surface and bottom heat exchange and is therefore nonconservative, it is not the ideal parameter for calibrating such models. Salinity, which is a conservative substance, has historically been regarded as the ideal constituent for hydrodynamic calibration involving advection and dispersion. However, this is generally feasible only for estuarine applications where salinity is routinely monitored (Cole and Wells, 2003). Because suspended solids containing substances that may decay 
and settle are not conservative, they are generally not a good substitute for salinity during calibration except in water-bodies where the conservative assumption is appropriate. In reality, there is no ideal constituent that can be used for hydrodynamic calibration.

Thermal mixing, advection by transport mechanism and dispersion by a combination of the effects of shear and diffusion are the most important aspects of the physical processes in a reservoir. Advection-dispersion (AD) models can normally be used to determine the spreading of neutrally buoyant substances such as organic compounds and suspended solids in a deep reservoir. Generally, water quality models require a coupling to $\mathrm{AD}$ and hydrodynamic models in order to calculate flows in which horizontal or vertical density gradients exist due to a conservative substance or temperature variation. In other words, the traditional approaches to water quality assessment are limited to the requirements of a detailed hydrodynamic study. This has led to water quality managers and limnologists to seek refuge in mathematical modelling applications (Chapra, 1997).

The links between external forcing, basin scale circulations, internal waves, intrusions, and turbulence may be considered in terms of paths along which energy fluxes connect these processes. Therefore, a lake is conceptualized as a system partitioned in terms of energies, instead of physical locations. Mechanical energy enters through external forcing, mostly at basin scales, and can only leave as heat by viscous dissipation, mostly at very small scales.

\subsubsection{Density Current}

The formation of density currents was first observed in some of the world's large reservoirs at the beginning of the last century (Basson, 1998). It is apparent that density differences often exist between the flows from tributaries and receiving water of the reservoirs. These differences generate density flows in the reservoirs (see Figure 3.2). If local mixing is inadequate in the region of the inflow to eliminate the density differences, the density or turbidity current will plunge and travel along the sloping bottom as an underflow.

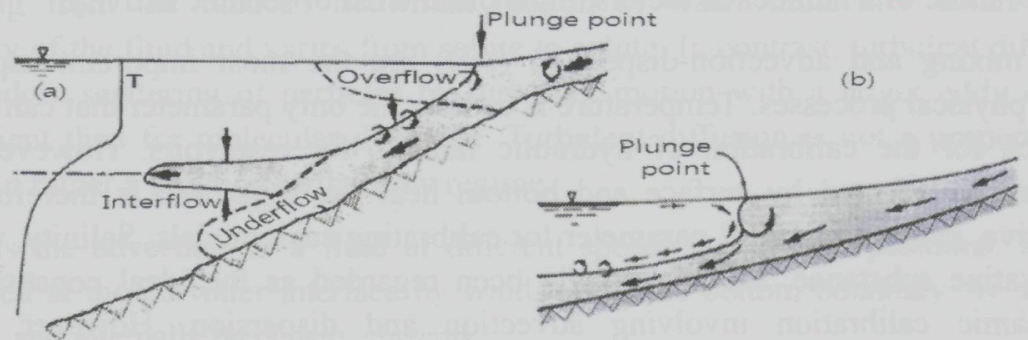

Figure 3.2. Density flows reservoirs in reservoirs from Moore and Thornton (1988): (a) Different density inflows to impoundment, (b) Pooling at the plunge point. 
This process is accompanied by the entrainment of ambient water. If the density of the inflow is less than the surface layer, the inflow will tend to travel along the free surface, thus entering as an over-flow. Interflow occurs when the density of the inflow is intermediate to that of a stratified reservoir. In this case, the inflow is distributed to an intermediate layer of similar density. An interflow may also move through a reservoir quickly if water is being extracted at a mid-level. An extraction discharge at low level may effectively trap the interflow within the impoundment. Water quality changes relate to the depth of the interflow, temperature, and residence time.

The velocity of the density current head in the expansion zone where a river enters a dam reservoir is a key parameter for evaluating the extent to which the suspended material travels, and for determining the type and distribution of sediment in the water body. Along the upper reaches of a reservoir, stable, floating debris is observed, indicating the so-called "plunge point" where the inflowing river stream changes into a density current. After flood flow the stationary position of the debris is caused by the slow upstream movement (of near zero velocity) of the overlying water mass, just downstream of the point where the small soil particle-laden inflow dives below the stored water mass.

Density currents can radically change the hydraulic regime and processes occurring in a reservoir to be different from the general characteristics described above, and therefore have a substantial effect on water quality. The plunging inflow phenomenon can be of concern for water quality issues associated with contaminants, pollutants and turbidities that may be carried in the density currents. This fact can greatly alter the assumptions of standard loading models (Kimmel and Groeger, 1984; Gaugush, 1986; Walker, 1987).

The presence or absence of light (which is a function of depth and water clarity) is vitally important for the water quality within an interflow. In particular, in the case of the formation of suspended solids density currents, the siltation time of the reservoir increases. Basson (1998) reviewed the following theory to predict density current formation using minimum stream power principles to derive the density current through a reservoir at the plunge point.

\section{1) Density current formation}

The velocity reaches a minimum and the water depth a maximum value at the plunge point from Figure 3.3.

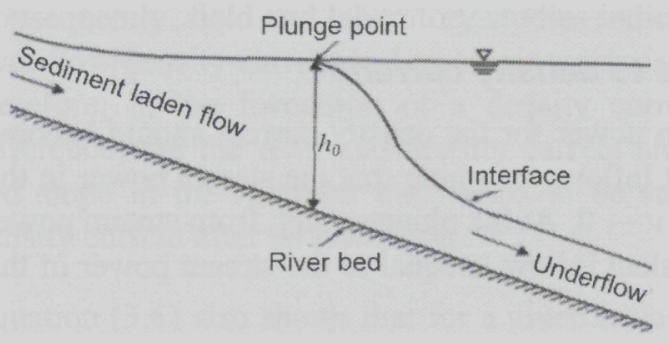

Figure 3.3. Schematic diagram of density current formation (Fan, 1960). 
Fan (1960) therefore proposed the specific energy as:

$$
E=h^{\prime}+\frac{v^{2}}{2 \frac{\Delta \gamma}{\gamma} g h^{\prime}}
$$

Equation (3.1) has a minimum value at the plunge point. Therefore if we differentiate $E$ with respect to $h^{\prime}$ :

$$
\frac{d E}{d h^{\prime}}=1-\frac{q^{2}}{\frac{\Delta \gamma}{\gamma} g h_{0}^{3}}=0
$$

where $h^{\prime}=$ density current flow depth

$v=$ mean velocity of a density current

$\gamma=$ specific receiving water weight

$\Delta \gamma=$ specific weight difference between density current and receiving water

$g=$ gravitational acceleration

$q=$ unit discharge

$h_{0}=$ depth at plunge point

Therefore, if the velocity at the plunge point is $v_{0}$ :

$$
\frac{v_{0}}{\sqrt{\frac{\Delta \gamma}{\gamma} g h_{0}}}=1
$$

This parameter is known as the densimetric Froude number $\left(F r_{D}\right)$. It is found however, that the densimetric Froude number also depends on the inflow sediment concentration and decreases with increasing concentration.

This explains why different researchers have found from their experiments such a wide range of densimetric Froude numbers that predict density current formation. According to the results of many researchers, the general variation of observed values is $0.1<F r_{D}$ $<0.9$, which shows the unreliability and site specificity of prediction of density current formation.

\section{2) Minimum stream power principle to density current}

At the plunge point, the reservoir stream power for the density current should become equal to or less than that of the turbulent inflow, assuming that the stream power in the upper layer is approximately zero where $v \rightarrow 0$. At the plunge point, from stream power continuity, the stream power of the turbulent inflow is equal to the stream power of the density current: 


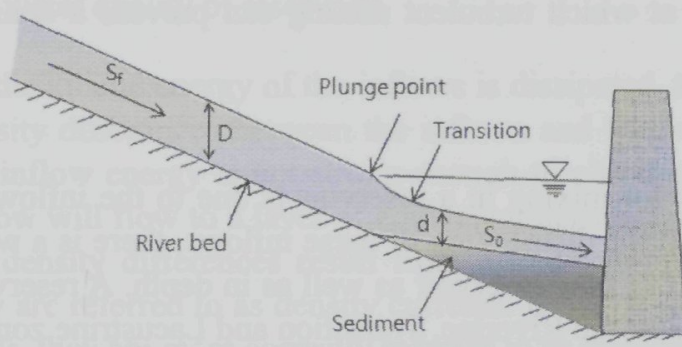

Figure 3.4. Plunge point characteristics.

$$
\int_{y_{0}}^{D} \rho g \bar{v} s_{f}=\int_{y_{0}}^{d} \Delta \rho g \bar{v} s_{0}
$$

Therefore, for a density current to form it is expected that:

$$
\frac{\Delta \rho g Q s_{0}}{\rho g Q s_{f}} \leq 1
$$

From continuity this simplifies to

$$
\frac{\Delta \rho s_{0}}{\rho s_{f}} \leq 1
$$

As a preliminary test of Equation (3.6), laboratory data of Akiyama et al. (1987) was used, in which the density difference was created by temperature differences. Verification of Equation (3.6) to test for density current formation does indeed show that less stream power is used by the density current than by turbulent open channel inflow. Furthermore, the densimetric Froude number (at plunge point) does not provide an accurate means of predicting density current formation due to the large scatter in values. The general trend indicates a lower density current stream power value as the densimetric Froude number increases.

Even if the stream power in the layer above the density current is added to that of the density current stream power, the difference will be negligible due to the small slope (due to the reservoir depth) and low velocity in the upper layer.

In short, from Equation (3.6), larger density differences, larger slope $s_{o}$, and smaller slope $s_{f}$ (of reservoir) will provide better conditions for density current formation. Consequently, field and laboratory studies indicate that the density differences between river flow/density current and reservoir water can be very small. The dominant variables, therefore, in the formation of a density current (given that there is some density difference) are the river and density current slope differences. On the other hand, the bed slope in the reservoir still needs to be steep enough for the propagation of the density current after its formation.

Equation (3.6) also shows that for a given inflow river slope, the reservoir bed slope at 
the plunge point has an upper limit at which turbulent mixing can prevent a density current from forming.

\section{3) Inflow Mixing Processes}

One of the primary forcing functions for mixing in a reservoir is due to the inflow of water into a lake or a reservoir. Due to the distribution of these inflows, there is a wide variation in the mixing along the length of the reservoir as well as in depth. A reservoir can be separated into three distinct zones; the Riverine, Transition and Lacustrine zones.

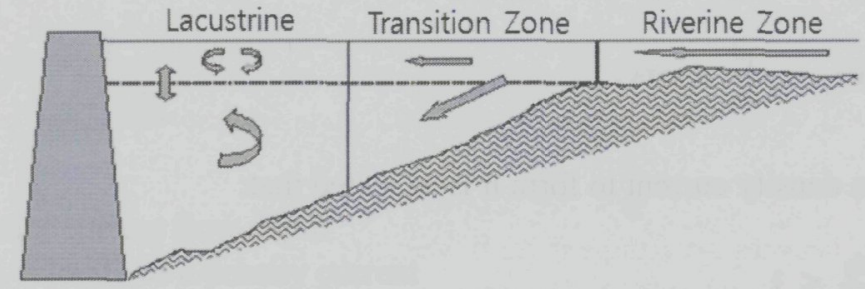

Figure 3.5. Mixing Zones in a Reservoir (Martin and MacCutcheon, 1999).

In the riverine zone, the inflows dominate the mixing and the water column is typically well-mixed. The transition zone is characterised by the transition from riverine-like to lake-like characteristics. In this zone, buoyancy forces due to differences in densities between inflows and the reservoir water begin to dominate the advective forces of the inflows. Within the transition zone, velocities decrease and the denser particles settle. Finally, in the lacustrine zone (central part of a lake) buoyancy forces dominate the flow patterns.

Inflows contribute to the mixing of lakes and reservoirs, and serve as a primary source of dissolved and particulate materials. The inflow rate and the density differences between the inflowing water and the lake water dictate their relative importance.

The initial momentum of an inflow into a reservoir pushes the more stagnant reservoir water ahead of it. The inflow continues to push the lake water ahead until the initial momentum is substantially dissipated by the river bottom shear forces and by the pressure gradient across the interface between the water masses. Prior to dissipation, the advective riverine processes dominate the transport within the reservoir. The turbulent kinetic energy within the reservoir is usually sufficient to keep the waters completely mixed vertically and to prevent the settling of materials. The mixing between the riverine inflow and the reservoir waters is limited. The quality and identity of the water is similar to that of the riverine water. The zone of the reservoir where riverine flows dominate the water characteristics is the riverine zone.

If the kinetic energy due to these inflows is large compared to the density gradient in the reservoir, the inflows can cause the whole reservoir to mix completely. Many weakly stratified reservoirs are often completely mixed during high-flow events. Where the inflows do not cause complete mixing, there can be still strong impact on circulation 
and water quality of reservoirs.

As the kinetic energy of the inflows is dissipated, the potential energy of inflows due to density differences between the inflows and lake water begin to dominate transport. If the inflow energy is not strong enough to eliminate these density differences, then the inflow will flow to a layer of equivalent density level and move along that layer. Where the density differences result from differences in temperature or dissolved materials, they are referred to as density currents. If the density differences result from particulate loads, they are more correctly referred to as turbidity currents.

If the riverine water is less dense (more buoyant) than the surface waters of the lake or reservoir, the more buoyant river water will flow over the top of the reservoir water as an over-flow. Over-flows are common in spring where the shallower river waters generally heat more rapidly than the reservoir waters.

Over-flows contribute significantly to water quality variations, since they add materials directly to the more productive surface zones of the lake or reservoir. Since over-flows tend to move over the top of the reservoir waters, the increased water surface creates a hydrostatic instability, which will tend to cause the over-flow to spread out quickly as it moves down the reservoir. The actual amount of spreading that occurs will be influenced by processes such as wind-mixing which can tend to mix the over-flow with underlying waters or push it to one side of the reservoir. Surface winds often cause overflows to be dissipated rapidly.

If the river water is denser than the surface water, the negative buoyancy causes the inflow to plunge beneath the lake or reservoir, becoming an underflow. The higher density can result in cooler inflow temperatures or higher concentrations of dissolved or particulate materials. The point where the underflows plunge (plunge point) or the separation point where over-flows detach from the bottom, is called the transition point between turbulent open channel or riverine inflows and the stratified flows in reservoirs. The transition point or the head of the reservoir does not remain stationary. As flows change, the plunge point and the separation point move upstream or downstream, defining a transition zone.

Underflows are common in autumn or fall. Shallow riverine water cools faster than the reservoir waters, and autumn storms cause higher turbidity inflows. In reservoirs, underflows will tend to follow the deepest channel, spreading out and dissipating due to turbulence generated by the bottom shear. In reservoirs, underflows tend to follow the drowned river channel. Velocities will decrease as the underflow moves along the bottom, due to shear between the flow and the bottom. As the current velocities decrease, suspended solids may settle out, causing turbidity currents to lose their identity. However, in reservoirs with relatively steep bottom slopes, turbidity currents may flow downward to the dam, resulting in problems with the design and operation of outlet structures. Density currents may also reach the base of the dam but generally do not cause problems like the sedimentation that occurs when turbidity current reaches the 
outlet structures.

If underflows are denser than the bottom waters, they may remain on the bottom. However, most underflows are less dense than the bottom-most layer. In such a case, the underflow separates from the bottom as an interflow (either density or turbidity). Interflows are not affected by bed shear, as with underflows. Surfaces of discontinuity in velocity and density form along the top and bottom of the interflow. Turbulent eddies mix and entrain reservoir waters. In large reservoirs, interflows may be affected by Coriolis force, which tends to deflect them to the right in the northern hemisphere.

Underflows, interflows and over-flows will typically move down-reservoir as long as additional riverine inflows of similar characteristics can push it forward. In reservoirs, the formation and movement of underflows and interflows may be enhanced by surface releases above the thermocline.

In general, however, underflows and interflows add oxygen-consuming materials to the more poorly mixed and less productive regions of the reservoir, thereby contributing to dissolved oxygen depletion in bottom waters. Density differences result in an effective isolation of the riverine waters so they become anoxic and chemically reduced. Interflows and underflows are often a major source of reduced materials to downstream reservoir regions. Alternatively, if the underflows are well oxygenated, they may improve the bottom water quality.

\subsubsection{Turbidity Occurrence}

Because of the quiescent nature of a reservoir, turbidity levels are generally lower compared to streams or rivers due to the settling of particles. In general, larger reservoirs or lakes have lower turbidity levels, usually below $100 \mathrm{NTU}$, whereas rivers can have turbidity values reaching over 1000 NTU (Basson, 1998). Lakes and reservoirs provide longer detention times, allowing for adequate settling of the larger turbid particles and suspended solids. A river inlet may be subject to greater swings in turbidity since they experience carryover of the river turbidity into the reservoir before the settling occurs.

High turbidity events in reservoirs occur in smaller reservoirs or lakes that receive water from agricultural watershed or urban drainage area. Larger reservoirs also experience high turbidity because of water quality changes during annual thermal changes in the lake, and may experience high turbidity events associated with severe flooding. During floods generated by heavy watershed runoff, smaller reservoirs are usually not large enough to settle out effectively the turbidity particles before they reach the dam site. In addition, if the reservoir is shallow, wave action created by high winds can stir up sediments from the bottom and re-suspend particles (US EPA, 1999). In larger thermally stratified reservoirs, turbidity current is generated due to a dense inflow, and can be long lasting due to the long retention time to settle out turbidity particles. For colloidal 
particles below $1 \mu \mathrm{m}$, this is because Brownian motion is the main mechanism of particle movement (Hofmann and Filella, 1999).

Reservoir turbidity events can also be caused by seasonal turnover of thermally stratified water in the reservoirs. This overturning effect can happen quickly and may bring anoxic water that is nutrient rich from the lower depths to the surface where algae are present. Sudden algal blooms can then severely raise turbidity levels in spring.

\subsection{Scales of Motions}

Fluid flows can be categorised in many ways. One of the principal divisions is between laminar and turbulent flows. In a laminar flow, the fluid paths are regular and smooth. Turbulence is flow dominated by recirculation, eddies, and apparent randomness. Flow in which turbulence is not exhibited is called laminar. It should be noted, however, that the presence of eddies or recirculation does not necessarily indicate turbulent flow, because these phenomena may be present in laminar flow as well. Turbulent flows often represented via Reynolds decomposition are broken down into the sum of average and fluctuating components (Deissler, 1992).

Fully turbulent flow has a high Reynolds number, $R e=U L / v$. Turbulent flows are those that have velocity and length scales, swirls (three-dimensional vortex) or eddies (twodimensional vortex) of different sizes. Turbulence can therefore be considered to consist of eddies of different sizes. The largest eddies arise from the velocity difference between the flow layers. These large eddies break down into small eddies. Finally, the smallest eddies break down and dissipate to heat due to viscosity. The size ratio between the smallest and largest eddies can be large, and depends on the Reynolds number.

An eddy cannot have a precise definition, but it is conceived to be a turbulent motion, localized over a region of size $l$ that is at least moderately coherent over the region. The region occupied by a larger eddy can also contain smaller eddies. Eddies of size $l$ have a characteristic velocity $u(l)$ and timescale $t(l) \equiv l / u(l)$. Eddies in the largest size range are characterized by the length scale $l_{0}$, which is comparable to the flow length scale $L$. Their characteristic velocity $u_{0} \equiv u\left(l_{0}\right)$ is the order of the RMS (root mean square) turbulence intensity (velocity) $u^{\prime} \equiv(2 \mathrm{k} / 3)^{1 / 2}$ which is comparable to $U$. Here the turbulent kinetic energy is defined as (Marshall and Bakker, 2003, Bakker, 2006):

$$
k=\frac{1}{2}\left\langle u_{i} u_{i}\right\rangle=\frac{1}{2}\left(\overline{u^{\prime 2}}+\overline{v^{\prime 2}}+\overline{w^{\prime 2}}\right)
$$

where « denotes an averaging operator (usually time average).

The Reynolds number of these eddies $R e_{0} \equiv u_{0} l_{0} / v$ is therefore large (comparable to $R e$ ) and the direct effect of viscosity on these eddies is negligibly small. 
These turbulent flows can be categorized according to three main turbulent length scales as shown in Figure 3.6: the integral scale, the Taylor scale, and the Kolmogorov scale, with their corresponding Reynolds numbers (Sanford, 1997; Bakker, 2006). Where the bulk of the energy is contained in the larger eddies in the size range $l_{E I}=l_{0} / 6<l<6 l_{0}$; this is called the energy-containing range. The suffixes $E I$ and $D I$ indicate that $l_{E I}$ is the demarcation line between energy $(E)$ and inertial $(I)$ ranges, as $l_{D I}$ is that between the dissipation $(D)$ and inertial $(I)$ ranges.

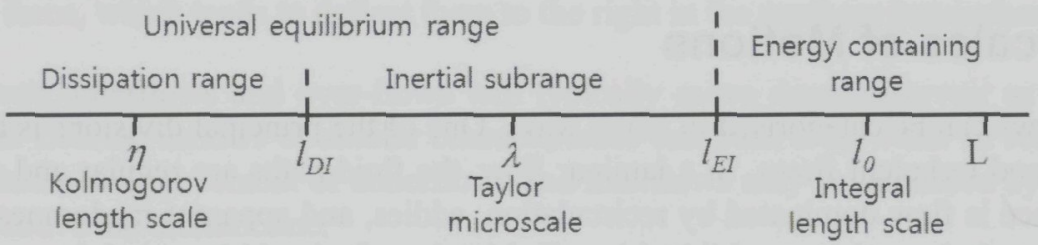

Figure 3.6. Turbulent ranges according to eddy size.

\subsubsection{Energy Containing Range}

An estimate of the length scale $l_{0}$ of the larger eddies can be derived as follows. Eddies of size $l_{0}$ have a characteristic velocity $u_{0}$ and timescale $t_{0} \equiv l_{0} / u_{0}$. Their characteristic velocity $u_{0} \equiv u\left(l_{0}\right)$ is the order of the RMS turbulence intensity (velocity) $u^{\prime} \equiv(2 \mathrm{k} / 3)^{1 / 2}$. Assume that energy of eddy with velocity scale $u_{0}$ is dissipated in time $t_{0}$. Therefore, we can then derive the following equation for this length scale:

$$
l_{0} \propto \frac{k^{3 / 2}}{\varepsilon}
$$

Here, $\varepsilon\left[\mathrm{L}^{2} / \mathrm{T}^{3}\right]$ is the energy dissipation rate. This length scale is usually referred to as the integral length scale of turbulence. The Reynolds number associated with these large eddies is referred to as the turbulence Reynolds number $R_{L}$, which is defined as:

$$
\operatorname{Re}_{L}=\frac{k^{1 / 2} l_{0}}{v}=\frac{k^{2}}{\varepsilon v}
$$

The large eddies are unstable and break up, transferring their energy to somewhat smaller eddies. These smaller eddies undergo a similar break-up process and transfer their energy to yet smaller eddies. This energy cascade - in which energy is transferred to successively smaller and smaller eddies - continues until the Reynolds number $\operatorname{Re}(l)$ $\equiv u(l) l / v$ is sufficiently small such that the eddy motion is stable, and molecular viscosity is effective in dissipating the kinetic energy. At these small scales, the kinetic energy of turbulence is converted into heat. 


\subsubsection{Universal Equilibrium Range}

The size range, $l<l_{E I}$, is referred to as the universal equilibrium range. In this range, the timescales $l / u(l)$ are small compared to $l_{0} / u_{0}$ so that the small eddies can adapt quickly to maintain dynamic equilibrium with the energy transfer rate imposed by the large eddies. On these scales, all high Reynolds number flow fields are statistically identical if the Kolmogorov scales scale the flow fields.

When we use the relationship $l_{0} \sim k^{3 / 2} / \varepsilon$ and substitute it for $l_{0}$ in the equations for the Kolmogorov scales, we can calculate the ratios between the small scale and large scale eddies:

$$
\begin{aligned}
& \eta / l_{0} \sim \operatorname{Re}_{L}^{-3 / 4} \\
& \left.\begin{array}{ll}
u_{\eta} / u_{0} & \sim \operatorname{Re}_{L}^{-1 / 4} \\
t_{\eta} / t_{0} & \sim \operatorname{Re}_{L}^{-1 / 2}
\end{array}\right\}
\end{aligned}
$$

As expected, at high Reynolds numbers, the velocity and timescales of the smallest eddies is small compared to those of the largest eddies. Since $\eta / l_{0}$ decreases with increasing Reynolds number, at high Reynolds number there will be a range of intermediate scales $l$ which is small compared to $l_{0}$ and large compared with $\eta$.

\section{1) Kolmogorov scales}

Kolmogorov proposed a transport equation for the turbulent kinetic energy and for the specific dissipation rate. It is based on dimensional analysis to provide a characteristic velocity and a characteristic length.

The Kolmogorov scale answers the following two questions: what is the size of the smallest eddies that are responsible for dissipating the energy? In addition, as $l$ decreases, do the characteristic velocity and timescales $u(l)$ and $t(l)$ increase, decrease, or stay the same? The assumed decrease of the Reynolds number $u_{0} l_{0} / v$ by itself is not sufficient to determine these trends.

These are answered by the Kolmogorov theory of turbulence (Pope, 2000); Kolmogorov turbulence theory describes how energy is transferred from larger to smaller eddies, how much energy is contained by eddies of a given size, and how much energy is dissipated by eddies of each size.

Given the two parameters $\varepsilon$ and $v$, we can form the following unique length, velocity, and time scales:

$$
\text { length scale: } \quad \eta=\left(v^{3} / \varepsilon\right)^{1 / 4}
$$




$$
\text { time scale: }\left\{\begin{array}{l}
t_{\eta}=(v / \varepsilon)^{1 / 2} \\
u_{\eta} / \eta=1 / t_{\eta} \\
R e_{\eta}=\eta u_{\eta} / v=1
\end{array}\right.
$$

These scales are indicative of the smallest eddies present in the flow, the scale at which the energy is dissipated. Note that the fact that the Kolmogorov Reynolds number $R e_{\eta}$ of the small eddies is 1 , is consistent with the notion that the cascade proceeds to smaller and smaller scales until the Reynolds number is small enough for dissipation to be effective.

\section{2) Taylor microscale}

For eddies in the inertial subrange of size $l$ using $\varepsilon=u_{\eta}{ }^{3} / \eta=\eta^{2} / t_{\eta}{ }^{3}$ and the previously shown relationships between the turbulent Reynolds number and various scales, velocity scales and timescales can be formed from $\varepsilon$ and $l$ :

$$
\left.\begin{array}{l}
u(l)=(\varepsilon l)^{1 / 3}=u_{\eta}(l / \eta)^{1 / 3} \sim u_{0}\left(l / l_{0}\right)^{1 / 3} \\
t(l)=\left(l^{2} / \varepsilon\right)^{1 / 3}=t_{\eta}(l / \eta)^{2 / 3} \sim t_{0}\left(l / l_{0}\right)^{2 / 3}
\end{array}\right\}
$$

A consequence, then, of the second similarity hypothesis (Bakker, 2006) is that in the inertial subrange the velocity scales and timescales $u(l)$ and $t(l)$ decrease as $l$ decreases. The energy dissipation rate $\varepsilon$ is given by the following equation, which comes from the analytically derived conservation equation for turbulent kinetic energy:

$$
\overline{S_{i j} S_{i j}} \gg S_{i j} S_{i j} ; \quad \varepsilon=2 v \overline{S_{i j} S_{i j}} ; \quad S_{i j}=\frac{1}{2}\left[\frac{\partial u_{i}}{\partial x_{j}}+\frac{\partial u_{j}}{\partial x_{i}}\right]
$$

The subscript indicates the fluctuating components. The dissipation rate depends on the viscosity and a velocity gradient (shear) in the turbulent eddies. Working out this equation further for isotropic turbulence (mainly bookkeeping for all the terms) results in:

$$
\varepsilon=15 v \overline{\left(\partial u_{1} / \partial x_{1}\right)^{2}}
$$

We can now define the Taylor microscale $\lambda$ as follows:

$$
\overline{\left(\partial u_{1} / \partial x_{1}\right)^{2}} \equiv \overline{u_{1}^{2}} / \lambda^{2}=u^{\prime 2} / \lambda^{2}
$$

This then results in the following relationship for the Taylor microscale $\lambda$ : 


$$
\varepsilon=15 v u^{\prime 2} / \lambda^{2}
$$

From $k=(1 / 2)\left(u^{\prime 2}+v^{\prime 2}+w^{\prime 2}\right)$ we can derive $k=(3 / 2) u^{\prime 2}$, and:

$$
\lambda \approx(10 v k / \varepsilon)^{1 / 2}
$$

The Taylor microscale falls in between the large scale eddies and the small scale eddies, which can be seen by calculating the ratios between $\lambda$ and $l_{0}$ and $\eta$ :

$$
\left.\begin{array}{l}
\lambda / l_{0}=\sqrt{10} R e_{L}^{-1 / 2} \\
\eta / l_{0}=\sqrt{10} R e_{L}^{-3 / 4} \\
\lambda / \eta=\sqrt{10} R e_{L}^{1 / 4} \\
\lambda=\sqrt{10} \eta^{2 / 3} l_{0}^{1 / 3}
\end{array}\right\}
$$

A commonly used quantity in the characterization of turbulence is the Taylor-scale Reynolds number $R_{\lambda}$. This is based on the length scale $\lambda$ and the corresponding velocity scale:

$$
R_{\lambda}=u^{\prime} \lambda / v
$$

$R_{\lambda}$ can be related to the turbulence Reynolds number as follows:

$$
R_{\lambda}=\left(\frac{20}{3} R e_{L}\right)^{1 / 2}
$$

We can also relate the timescale of the eddy of length scale $\lambda$ to the Kolmogorov timescale:

$$
\lambda / u^{\prime}=(15 v / \varepsilon)^{1 / 2}=\sqrt{15} t_{\eta}
$$

Finally, Reynolds numbers and turbulent length scales are summarised as following equations. The following Reynolds numbers have been defined:

Flow Reynolds number: $\quad R e=U L / v$

Turbulence Reynolds number: $R e_{L}=k^{2} / \varepsilon v$

Taylor Reynolds number: $R e_{\lambda}=u^{\prime} \lambda / v$

Kolmogorov Reynolds number: $R e_{\eta}=\eta u_{\mu} / v=1$ )

The flow Reynolds number is of the order of one to ten times the turbulence Reynolds number. 


\subsection{Navier-Stokes Equation}

\subsubsection{Definition}

The vast majority of work on the Navier-Stokes equations is done under an incompressible flow assumption for Newtonian fluids. Therefore, the equations can be used to solve different types of flows, including both laminar and turbulent flows at the scale of interest. It is assumed that the fluid is a continuum, i.e., is not made up of discrete particles. Another necessary assumption is that all the fields of interest such as pressure, velocity, density, temperature etc. are differentiable (i.e. no phase transitions).

The equations are derived from the basic principles of conservation of mass, momentum and energy. In solving the equations, it is sometimes necessary to consider a finite arbitrary volume, called a control volume. Fortunately, with modern computers it is comparatively straightforward to solve the Navier-Stokes equations numerically for turbulent flow while making the necessary assumptions. Considering the incompressible flow assumption and assuming a constant viscosity, the Navier-Stokes equations dictating not position but rather velocity become (Rhyming, 1991; Wikipedia, 2009).

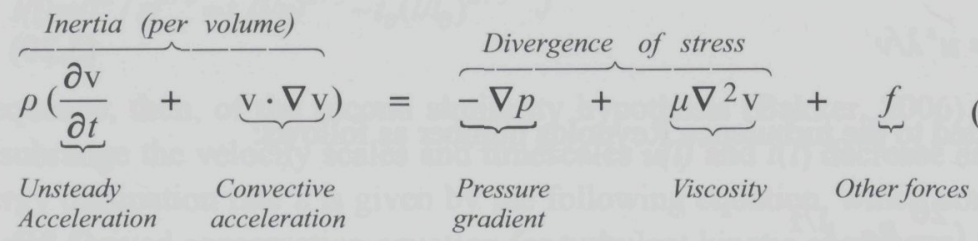

where $\nabla$ is partial derivative operator, $f$ represents "other" body forces (forces per unit volume), such as gravity or centrifugal force. Note that only the convective terms are nonlinear for incompressible Newtonian flow. The convective acceleration is an acceleration caused by a (possibly steady) change in velocity over position, for example the speeding up of fluid entering a converging nozzle. Though individual fluid particles are being accelerated and thus are under unsteady motion, the flow field (a velocity distribution) will not necessarily be time dependent.

Another important observation is that the viscosity generates a diffusion of momentum. If temperature effects are also neglected, the only other needed equation (apart from initial/boundary conditions) is the mass continuity equation. Under the incompressible assumption, density is a constant and it follows that the equation will simplify to $\nabla \cdot v=0$

The equations are commonly used in 3-coordinate systems: Cartesian, cylindrical, and spherical. The Cartesian equations follow directly from the vector equation above. Writing the vector equation explicitly, 


$$
\left.\begin{array}{l}
\rho\left(\frac{\partial u}{\partial t}+u \frac{\partial u}{\partial x}+v \frac{\partial u}{\partial y}+w \frac{\partial u}{\partial z}\right)=-\frac{\partial p}{\partial x}+\mu\left(\frac{\partial^{2} u}{\partial x^{2}}+\frac{\partial^{2} u}{\partial y^{2}}+\frac{\partial^{2} u}{\partial z^{2}}\right)+\rho g_{x} \\
\rho\left(\frac{\partial v}{\partial t}+u \frac{\partial v}{\partial x}+v \frac{\partial v}{\partial y}+w \frac{\partial v}{\partial z}\right)=-\frac{\partial p}{\partial y}+\mu\left(\frac{\partial^{2} v}{\partial x^{2}}+\frac{\partial^{2} v}{\partial y^{2}}+\frac{\partial^{2} v}{\partial z^{2}}\right)+\rho g_{y} \\
\rho\left(\frac{\partial w}{\partial t}+u \frac{\partial w}{\partial x}+v \frac{\partial w}{\partial y}+w \frac{\partial w}{\partial z}\right)=-\frac{\partial p}{\partial z}+\mu\left(\frac{\partial^{2} w}{\partial x^{2}}+\frac{\partial^{2} w}{\partial y^{2}}+\frac{\partial^{2} w}{\partial z^{2}}\right)+\rho g
\end{array}\right\}
$$

Note that gravity has been accounted for as a body force, and the values of $g_{x}, g_{y}, g_{z}$ will depend on the orientation of gravity with respect to the chosen set of coordinates. Here the velocity components (the dependent variables to be solved for) are $u, v$, and $w$. The continuity equation becomes:

$$
\frac{\partial u}{\partial x}+\frac{\partial v}{\partial y}+\frac{\partial w}{\partial z}=0
$$

This system of four equations comprises the most commonly used and studied form. Although comparatively more compact than other representations, this is a nonlinear system of partial differential equations for which solutions are difficult to obtain (Wikipedia, 2009).

\subsubsection{Methods of Solution}

The Navier-Stokes equations need to be discretised in both time and space in order to yield a solution (Alvelius, 1999). This has led to different methods to make NavierStokes equations less sensitive to the different size of the scales. In one of these methods, a statistical approach is employed, which considers an ensemble averaged field (Alvelius, 1999). This approach is one of the most accepted but different from solutions that are typically trajectories of position of a particle or deflection of a continuum. The methods used to solve the Navier-Stokes equations include:

- Direct Numerical Simulation (DNS)

- Large Eddy Simulation (LES)

- Reynolds Averaged Navier Stokes (RANS) Simulations

\section{1) DNS}

A direct solution of the Navier-Stokes equations is called Direct Numerical Simulation (DNS), and solves the Navier-Stokes equations numerically without any assumptions made about the turbulence model. All the spatial scales of the turbulence must be resolved in the computational mesh, from the smallest dissipative scales (Kolmogorov length scale, $\eta$ ), up to the integral scale $L$, associated with the motions containing most 
of the kinetic energy.

On the other hand, the integral scale depends usually on the spatial scale of the boundary conditions. To satisfy these resolution requirements, the number $N$ of points along a given mesh direction with increments $h$, must be

$$
N h>L
$$

so that the integral scale is contained within the computational domain, and also

$$
h \leq \eta,
$$

so that the Kolmogorov scale can be resolved. Since

$$
\varepsilon \approx \frac{u^{\prime 3}}{L}
$$

where $u^{\prime}$ is the RMS velocity, the previous relations imply that a three-dimensional DNS requires a number of mesh points $N^{3}$ satisfying

$$
N^{3} \geq R e^{9 / 4}=R e^{2.25}
$$

where $R e$ is the turbulent Reynolds number:

$$
\operatorname{Re}=\frac{u^{\prime} L}{v}
$$

Hence, the memory storage requirement in a DNS grows very fast with the Reynolds number. In addition, given the very large memory necessary, the integration of the solution in time must be done using an explicit method. This means that in order to be accurate, the integration must be done with a time step, $\Delta t$, small enough such that a fluid particle moves only a fraction of the mesh spacing $h$ in each step; that is,

$$
C r=\frac{u^{\prime} \Delta t}{h}<1
$$

(here $\mathrm{Cr}$ is the Courant number). The total time interval simulated is generally proportional to the turbulence time scale $t$ given by

$$
t=\frac{L}{u^{\prime}}
$$

Combining these relations, and the fact that $h$ must be of the order of $\eta$, the number of time-integration steps must be proportional to $L /(\mathrm{Cr} \eta)$. On the other hand, from the definitions for $R e, \eta$ and $L$ given above, it follows that 


$$
\frac{L}{\eta} \sim R e^{3 / 4}
$$

and consequently, the number of time steps grows as a power law of the Reynolds number.

\section{2) LES}

LES solves the Navier-Stokes equations for large scale turbulent fluid flows and models only the small scale fluctuations (smaller than a computational cell). Because it is a transient formulation, the required computational resources are considerably larger than those required for the regional spectral (RS) and k- $\varepsilon$ style models. In addition, a finer grid is needed to gain the maximum benefit from the model, and to accurately capture the turbulence in the smallest, sub-grid scale eddies (SGS) (Marshall and Bakker, 2003, Bakker, 2006).

A common deduction of Kolmogorov's (1941) famous theory of self-similarity is that large eddies of the flow are dependent on the flow geometry, while smaller eddies are self similar and have a universal character. For this reason, it became a practice to solve only for the large eddies explicitly, and model the effect of the smaller and universal eddies on the larger ones. Thus, in LES the large scale motions of the flow are calculated, while the effect of the smaller universal scales (the so called sub-grid scales) are modeled using a sub-grid scale (SGS) model. In practical implementations, it is required to solve the filtered Navier-Stokes equations with an additional sub-grid scale stress term. The most commonly used SGS models are the Smagorinsky model and its dynamic variants (Liu et al., 1995). They compensate for the unresolved turbulent scales through the addition of an eddy viscosity into the governing equations.

\section{3) RANS}

The RANS equation is obtained from the Navier-Stokes equation for conservation of fluid dynamic momentum (an equation of motion) by decomposing the velocity into time-average velocity terms $\bar{u}$ and instantaneous deviation $u^{\prime}$ (Rood, 1996). It is primarily used for dealing with turbulent flows. These equations can be used with approximations based on knowledge of the properties of flow turbulence to give approximate averaged solutions to the Navier-Stokes equations. For an incompressible flow of Newtonian fluid, these equations can be written as

$$
\rho\left(\bar{u} \bar{u}_{x}+\bar{v} \bar{u}_{y}+\bar{w} \bar{u}_{z}\right)=-\bar{P}_{x}=\mu \nabla^{2} \bar{u}-\rho\left(\overline{u_{x}^{\prime 2}}+\left(\overline{u^{\prime} v^{\prime}}\right)_{y}+\left(\overline{u^{\prime} v^{\prime}}\right)_{z}\right)
$$

and similarly for the $y$ and $z$ components. The left hand side of Equation (3.35) represents the change in mean momentum of a fluid element due to the unsteadiness in the mean flow and the convection by the mean flow. This change is balanced by the mean body force, the isotropic stress due to the mean pressure field, the viscous stresses, and apparent stress $\left(\rho u^{\prime} u^{\prime} \& \rho v^{\prime} u\right)$ due to the fluctuating velocity field, generally referred to as the Reynolds stresses. 
The basic tool required for the derivation of the RANS equations from the instantaneous Navier-Stokes equations is the Reynolds decomposition described in Section 3.4. Reynolds decomposition refers to the separation of the flow variable (like velocity $u$ ) into the mean (time-averaged) component $(\bar{u})$ and the fluctuating component $(u)$.

In this approach, a large part of the information about the flow is averaged out, and encapsulated into the Reynolds stress tensor, which is unknown and needs to be modelled in terms of the averaged quantities. The models of this approach have to be calibrated in many different flow configurations, and sometimes model parameters have to be tuned for the specific problem type (Alvelius, 1999).

When modelling using the RANS approach, the models created lack generality: the model constants are usually set using a few simple flows. When the models are applied to flows that are very different from the ones used for calibration, the constants often have to be readjusted to yield accurate predictions (Piomelli and Chasnov, 1995). Further more, the laminar-turbulent transition is exceedingly difficult to predict using the RANS approach, and requires the addition of semi-empirical data, usually in form of intermittency functions that mean the alternation of phases of the laminar-turbulent transition. The main reason for lack of generality of turbulence models lies in the fact that the model must represent a very wide range of scales. While small scales are affected strongly by viscosity, the large ones are affected very strongly by boundary conditions.

LES requires less computational effort than direct numerical simulation (DNS) but more effort than those methods that solve the Reynolds-averaged Navier-Stokes equations (RANS). The computational demands also increase significantly near walls, and simulating such flows usually exceeds the limits of modern supercomputers today. For this reason, zonal approaches are often adopted, with RANS or other empirically based models replacing LES in the wall region. The main advantage of LES over computationally cheaper RANS approaches is the increased level of detail it can deliver. While RANS methods provide "averaged" results, LES is able to predict instantaneous flow characteristics and resolve turbulent flow structures (Wikipedia, 2009).

\subsubsection{Limit in Engineering Application}

In three-dimensional flows, the non-linear term in the Navier Stokes equations transfers energy from the large energy producing scales to the small dissipative scales. The largest scales in the flow are typically determined by the characteristic size of the flow domain, and the smallest scales are determined by the kinematic viscosity and the dissipation rate.

The large and small scales can formally be defined as the integral length scale (the largest length scale in a flow, or characteristic length of the flow) $L$ and the Kolmogorov micro length scale (the smallest length or turbulent micro scale in the flow) $\eta=\left(v^{3} / \varepsilon\right)^{1 / 4}$. Here $\varepsilon(=d k / d t)$ is turbulence kinetic energy dissipation rate with the dimensions of 
$\mathrm{L}^{2} \mathrm{~T}^{-3}$. The Reynolds Number represented as $R e=(L / \eta)^{4 / 3}$ is also the measure of the range of scales in the flow. The large and small length scales are associated with the corresponding large and small time scales respectively.

The number of grid points required for a full solution of turbulent flows must be at least $L / \eta$ (ratio of largest to the smallest eddies) in order to be able to describe all the scales of motion. Since the number of floating-point operations required to complete the simulation is proportional to the grid size, the cost of simulation is:

$$
\text { cost of simulation } \propto(L / \eta)^{4} \propto \operatorname{Re}^{3}
$$

Therefore, the cost of simulation rises rapidly with the Reynolds number. In most engineering applications, the Reynolds number is typically $10^{5} \sim 10^{8}$. This means that the cost of simulation is the order of $10^{15} \sim 10^{24}$ computational grids. Consequently, according to Equation (3.36) and (3.33), the number of time steps grows also as a power law of the Reynolds number.

Especially, the computational cost of DNS is very high, even at low Reynolds numbers. For the Reynolds numbers encountered in most industrial applications, the computational resources required by a DNS would exceed the capacity of the most powerful computers currently available. However, direct numerical simulation is a useful tool in fundamental research on turbulence. Using DNS it is possible to perform numerical experiments, and extract from them information difficult or impossible to obtain in the laboratory, allowing a better understanding of the physics of turbulence. In addition, direct numerical simulations are useful in the development of turbulence models for practical applications, such as sub-grid scale models for large eddy simulation (LES) and models for methods that solve the Reynolds-averaged NavierStokes equations (RANS).

\subsection{Reynolds Time-averaged Mean Flow and Transport Equations}

The equations developed in the previous Section 3.3 with the equation of state form a closed set of equations. This set of equations is exact when written with instantaneous variables (continuous in time). These equations can be solved with ease in several types of laminar flows. However, for most flows of practical relevance, these equations cannot be solved using an exact method. In natural waters, flows are always turbulent. In spite of the recent advances in computing, a general turbulent fluid motion cannot be calculated with an exact method (Martin and McCutcheon, 1999).

As a result, practical applications require other solution techniques such as described in Section 3.4 to reduce the basic equations to a more manageable form. One of these approaches is to describe the turbulent motion by deriving what is known as the Reynolds number of turbulent motion, which describes the ratio between convection and molecular diffusion $(R e=(\mathrm{v} \cdot d) / v$, where $\mathrm{v}$ is velocity, $d$ hydraulic radius and $v$ dynamic viscosity). 
In the RANS approach, the instantaneous velocity field is broken into a mean (ensemble average) value, which varies slowly, and a rapidly fluctuating component:

$$
u(x, y, z, t)=\bar{u}(x, y, z, t)+u^{\prime}(x, y, z, t)
$$

where the over-bar represents a mean value, the prime symbol represents the fluctuation or variation about the mean velocity (see Figure 3.7). This decomposition is called the Reynolds decomposition, which is a mathematical technique to separate the average and fluctuating parts of a quantity.

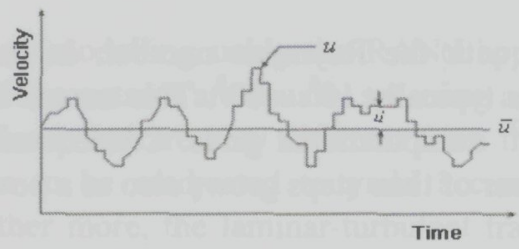

Figure 3.7. Velocity fluctuations at a fixed point

The ensemble average of a quantity at the grid point $i$, say the velocity, at a point $x$ and time $t$ is the arithmetic average of that quantity for all the flows (Deissler et al., 1992). For instance, for a velocity component $u_{i}$ at point $x$ and time $t$, the ensemble average entails that it is assumed that the statistical properties obtained by averaging over a set of realisations (ensemble average) coincide with those obtained by averaging a single realisation for a sufficiently long time (time average):

$$
\bar{u}_{i}(\vec{x}) \equiv \underbrace{\lim _{N \rightarrow \infty} \frac{1}{N} \sum_{n=1}^{N} u_{i}^{(n)}(x, t)}_{\text {Ensemble Average }} \approx \frac{1}{T}_{t-T / 2}^{t+T / 2} \int_{t / 2} u_{i}(x, t) d t
$$

where $n$ indicates the $n$th flow, $N$ is the total number of flows over which the average is taken, and $T$ is the interval, which should be taken to be (very) large compared to the correlation time of the turbulent velocity. The turbulent velocity $u_{i}^{\prime}(x, t)$ has zero mean, but deeply influences the kinetics of the mean flow.

All the physical quantities involved in the Navier Stoke equations are broken into a mean and fluctuating part, and substituted into the Navier Stokes equations. For completeness, note the sources/sinks terms in the equations must also be expressed in terms of mean and fluctuating values (Martin and McCutcheon, 1999). This kind of decomposition when applied to the whole set of equations, transforms the instantaneous variables into mean flow variables, as the independent variables. However, new terms are introduced into the equations, which are unknown, that is, the term $\overline{\rho u_{i}^{\prime} u_{j}^{\prime}}$. These terms are known as Reynolds's stresses.

An approach to model these terms is done through new sub-models, applied as part of the hydrodynamic models. These sub-models are known as turbulence models. 


\subsection{Turbulent Viscosity}

By applying the time-averaging procedure, the small-scale iluctuations are avoided. However, new terms, the Reynolds stresses are introduced into the momentum equations that are unknown, hence creating a closure problem of the RANS equations. These expressions have to be evaluated in order to close the set of governing equations; this is the task of turbulence modelling.

Statistically, the Reynolds Stress term $\overline{\rho u_{i}^{\prime} u_{j}^{\prime}}$ is a result of a compelling property of turbulent flows: the non-linearity of the Navier Stokes equations.

\subsubsection{The concept of turbulent viscosity (Eddy Viscosity)}

In the study of turbulence in fluids, a common practical strategy for calculation is to ignore the small-scale vortices (or eddies) in the motion and instead to calculate the large-scale motion with an eddy viscosity that characterizes the transport and dissipation of energy in the smaller-scale flow. Important features of turbulence can be described in terms of a turbulent viscosity (eddy viscosity). A concept, originally introduced by Boussinesq, provides a valuable simple contact with the dynamics of turbulence despite its numerical flaws.

This method is based on the analogy between momentum transport in the viscous, laminar flows and momentum transport in turbulent flows. A similar analogy is useful for molecular diffusion and turbulent transport of water quality constituents, and thermal diffusivity and the turbulent transport of heat energy.

The momentum equation is a force balance based on Newton's second law (law of acceleration: The rate of change of momentum of a body is proportional to the resultant force acting on the body and is in the same direction.). Consistent with the Newton's second law, the turbulent fluctuations must be stresses in the flow. Since the averages of the fluctuating terms represent a change in motion of the fluid transported by these fluctuations, these terms therefore represent a shear force or stress. Thus, the averages of fluctuations, when multiplied by density, physically represent the transport of momentum, heat and constituent mass due to turbulent fluctuating motion. These stress terms are usually called turbulent stresses or Reynolds stresses. For example, the stress terms in the $x$-direction momentum equation are

$$
\left.\begin{array}{l}
\tau_{x x}=-\rho \overline{u^{\prime} u^{\prime}} \\
\tau_{y x}=-\rho \overline{u^{\prime} v^{\prime}} \\
\tau_{z x}=-\rho \overline{u^{\prime} w^{\prime}}
\end{array}\right\}
$$

where $\tau_{x j}$ is stress in the $x$ direction resulting from velocity components acting in the $j$ 
direction $(j=x, y$, or $z)$ of the flow. The observation that the turbulent fluctuations are of the form of turbulent stresses provides a relationship between time-averaged flow variable $\tau_{i j}$ and the velocity fluctuations. The first method to derive the turbulent shear stress $\tau_{i j}$ was introduced by Boussinesq, who proposed that the turbulent stresses could be related to the mean strain $(\partial u / \partial y)$ by an apparent viscosity term: the turbulent or eddy viscosity. Boussinesq based the derivation of the eddy viscosity on an analogy with Newton's law of viscosity

$$
\tau=-\mu \frac{\partial u}{\partial z}
$$

where $\mu$ is the proportionality constant known as the coefficient of molecular viscosity with dimensions $\mathrm{ML}^{-1} \mathrm{~T}^{-1}$. Boussinesq proposed that turbulent shear stresses could be similarly described, as

$$
\tau_{z x}=-\rho \overline{u^{\prime} w^{\prime}}=-N_{z x} \frac{\partial u}{\partial z}
$$

where $N_{z x}$ is the coefficient eddy viscosity or turbulent viscosity, which like the molecular viscosity has dimensions $\left[\mathrm{ML}^{-1} \mathrm{~T}^{-1}\right]$. The subscripts refer to eddy viscosity in the vertical direction $(z)$ due to a vertical gradient of velocity acting in horizontal direction $(x)$. The SI Units of eddy viscosity are $\mathrm{Nsm}^{-2}$. In the same fashion, an expression for turbulent transport of constituent mass or thermal energy is developed using an analogy to molecular diffusion and thermal diffusivity as:

$$
-\overline{u^{\prime} C}=D_{x} \frac{\partial C}{\partial x},-\overline{v^{\prime} C}=D_{y} \frac{\partial C}{\partial y},-\overline{w^{\prime} C}=D_{z} \frac{\partial C}{\partial z},-\overline{u^{\prime} T}=K_{x} \frac{\partial T}{\partial x},-\overline{v^{\prime} T}=K_{y} \frac{\partial T}{\partial y},-\overline{w^{\prime} T^{\prime}}=K_{z} \frac{\partial T}{\partial x}
$$

where $D_{x}, D_{y}, D_{z}$ are coefficients of eddy or turbulent diffusion, while $K_{x}, K_{y}, K_{z}$ are the coefficients of turbulent thermal diffusivity. The Dimensions of both $D_{x}, D_{y}, D_{z}$ and $K_{x}$, $K_{y}, K_{z}$ are $\mathrm{L}^{2} \mathrm{~T}^{-1}$ and the SI units are $\mathrm{m}^{2} \mathrm{~s}^{-1}$.

The relationships for turbulent stresses are substituted into RANS, and the resulting expressions in the $x$-direction are diffusion terms.

$$
-\frac{\partial\left(\overline{u^{\prime} C^{\prime}}\right)}{\partial x}=\frac{\partial}{\partial x}\left(D_{x} \frac{\partial C}{\partial x}\right)
$$

Substitution will yield similar terms for the turbulent stresses and diffusive mixing terms as in Table 3.1. Generally, eddy viscosities and diffusivities are much greater than the molecular viscosities and diffusivities.

In water, the coefficients for turbulent viscosity and diffusivity are also much greater than molecular viscosity and diffusivity $\left(N_{i j} \gg \mu, D_{i} \gg D_{m}, K_{i} \gg k_{c}\right.$, where $k_{c}$ is 
thermal conductivity of water) so that the molecular terms are ignored in the basic equations. Only in the case of the vertical eddy viscosity, where stratification can completely dampen turbulence, does it approaches the value of kinematic viscosity.

Note a basic difference between molecular viscosity and turbulent momentum transfer is that molecular viscosity is a property of the fluid while turbulent eddy viscosity is a property of the flow (Martin, 1988).

While molecular coefficients are constant in all directions (isotropic) for a given temperature, the turbulent mixing coefficients are often not (non-isotropic). The turbulent transfer coefficients depend on the scale of turbulent motion, and thus require different values specified in each coordinate direction.

Table 3.1. Mathematical Descriptions of Hydrodynamic Flow Using Eddy Viscosity and Eddy Diffusion Relationships

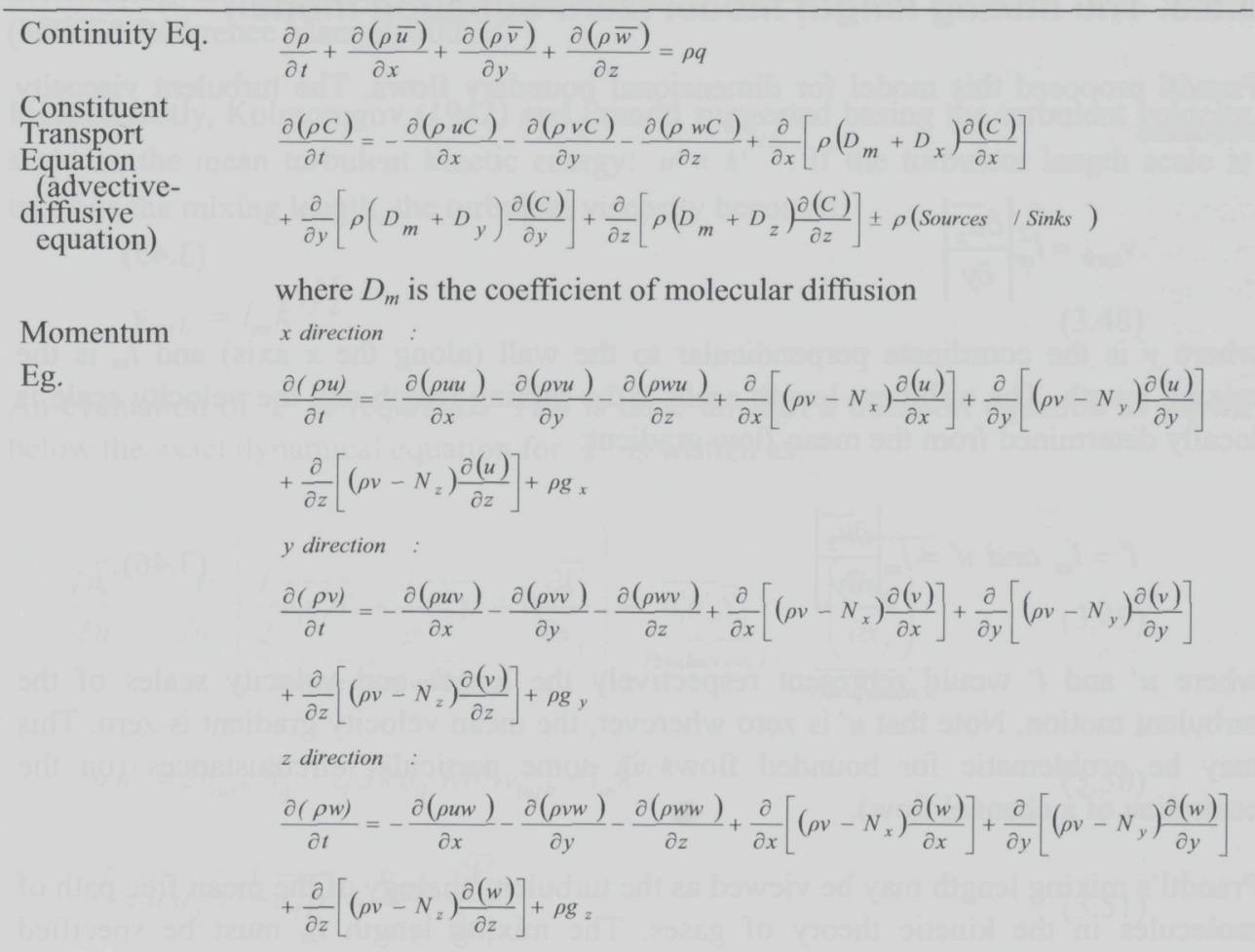

where $N_{\mathrm{s}}$ is horizontal viscosity, $N_{y}$ vertical viscosity, $N_{z}$ lateral viscosity.

Equation of State $\rho=f(C, T)$

\subsubsection{The magnitude of the turbulent viscosity}

Dimensionally, $v_{t u r b}(x, t)$ is equivalent to the product of a velocity and length scale. This 
suggests that by analogy of kinetic theory for gases,

$$
v_{\text {turb }}=u^{\prime} l^{\prime}
$$

where $u^{\prime}$ and $l^{\prime}$ would represent the velocity and length scales of turbulent motion. This dimensional analysis of turbulent viscosity is applied in evaluation of turbulent viscosity. A number of models have been developed, following this analogy of dimensional analysis, to evaluate the turbulent viscosity.

In the mixing length model, $u^{\prime}$ and $l^{\prime}$ are specified on the basis of the geometry of the flow. In two-equation models, the so-called $(k-\varepsilon)$ model, $u^{\prime}$ and $l^{\prime}$ are related to the turbulent kinetic energy and turbulent dissipation, for which modelled equations are explicated (Lévêque, 2005).

\subsubsection{The mixing length model (zero-equation model)}

Prandtl proposed this model for dimensional boundary flows. The turbulent viscosity becomes

$$
v_{t u r b}=l_{m}^{2}\left|\frac{\partial \overline{u_{x}}}{\partial y}\right|
$$

where $y$ is the coordinate perpendicular to the wall (along the $x$ axis) and $l_{m}$ is the mixing length. The turbulent length scale is the mixing length and the velocity scale is locally determined from the mean flow gradient:

$$
l^{\prime}=l_{m} \text { and } u^{\prime}=l_{m}\left|\frac{\partial \overline{u_{x}}}{\partial y}\right|
$$

where $u^{\prime}$ and $l^{\prime}$ would represent respectively the length and velocity scales of the turbulent motion. Note that $u^{\prime}$ is zero wherever, the mean velocity gradient is zero. This may be problematic for bounded flows in some particular circumstances (on the centreline of a channel flow).

Prandtl's mixing length may be viewed as the turbulent analogy of the mean free path of molecules in the kinetic theory of gases. The mixing length $l_{m}$ must be specified according to the geometry of the flow. This specification is usually empirical; it is derived from experiment and observation rather than theory.

A possible generalisation of the mixing length hypothesis is

$$
v_{\text {turb }}=l_{m}{ }^{2}|\bar{S}|
$$


where $|\bar{S}|^{2}=\sum_{i j} \bar{S}_{i j}{ }^{2}, \bar{S}_{i j} \equiv \frac{1}{2}\left(\frac{\partial \bar{u}_{i}}{\partial x_{j}}+\frac{\partial \bar{u}_{j}}{\partial x_{i}}\right)$ is the (symmetric) mean strain-rate tensor.

In its generalised form, this model is arguably the simplest model of turbulence. The mixing length model is valuable in simple flows such as shear layer or pressure driven flows, but it cannot account for the transport effects of turbulence.

\subsubsection{One-equation model: the k-model}

The $k$ turbulence model constitutes the first important improvement of the mixinglength theory by determining the velocity scale from a transport equation rather than from the mean flow field. $k$ is a measure of the intensity of the turbulent fluctuations (the turbulent kinetic energy) and thus physically an inherent quantity to include in determining the eddy viscosity as the energy is contained in the large-scale eddies (MIKE3 Reference Manual, 2005).

Independently, Kolmorogov (1942) and Prandtl suggested basing the turbulent velocity scale on the mean turbulent kinetic energy: $u^{\prime} \propto{\overline{k^{\prime}}}^{1 / 2}$. If the turbulent length scale is taken as the mixing length, the turbulent viscosity becomes

$$
v_{\text {turb }}=l_{m}, \bar{k}^{1 / 1} / 2
$$

An evaluation of $\overline{k^{\prime}}$ is required. This is done through a transport equation as shown below the exact dynamical equation for $\overline{k^{\prime}}$ is written as:

$$
\begin{aligned}
& \frac{D \overline{k^{\prime}}}{D t}=-\frac{\partial}{\partial x_{j}}(\underbrace{\frac{1}{2} \overline{u_{i}^{\prime} u_{i}^{\prime} u_{j}^{\prime}}+\frac{1}{\rho} \overline{u_{j}^{\prime} p^{\prime}}}_{\text {Turbulent Transport }}+v \frac{\partial \overline{k^{\prime}}}{\partial x_{j}})-\underbrace{\overline{u_{i}^{\prime} u_{j}^{\prime} \bar{S}_{i j}}}_{\text {Production:P }}-\underbrace{v \overline{\left(\frac{\partial u_{i}^{\prime}}{\partial x_{j}}\right)^{2}}}_{\text {Dissipation:E }} \\
& -\overline{u_{i}^{\prime} u_{j}^{\prime}} \approx 2 v_{\text {turb }} \cdot \bar{S}_{i j}-2 / 3 \bar{k}^{\prime} \delta_{i j} \text { with } v_{\text {turb }}=l_{m} \bar{k}^{1 / 2} \\
& -\frac{1}{2} \overline{u_{i}^{\prime} u_{i}^{\prime} u_{j}^{\prime}}+\frac{1}{\rho} \overline{u_{j}^{\prime} p^{\prime}} \approx \frac{v_{t u r b}}{\sigma_{k}} \frac{\partial \overline{k^{\prime}}}{\partial x_{j}}
\end{aligned}
$$

This derives from the gradient-diffusion hypothesis. The empirical coefficient $\sigma_{k}$ may be regarded as the turbulent Prandtl number (order of unity) for the kinetic energy. From a dimensional argument, the mean dissipation rate: $\bar{\varepsilon}=C \bar{k}^{3 / 2} / l_{m}$, where $C$ is a model constant. Finally, the transport equation for the mean turbulent kinetic energy is written as: 


$$
\frac{D \overline{k^{\prime}}}{D t}=-\frac{\partial}{\partial x_{j}}\left(\left(v+\frac{v_{t u r b}}{\sigma_{k}}\right) \frac{\partial \overline{k^{\prime}}}{\partial x_{j}}\right)+P-\bar{\varepsilon}
$$

with $v_{\text {turb }}=l_{m} \overline{k^{\prime}}$ and $\varepsilon=C \overline{k^{\prime}}$ /2/2 $l_{m}$ together with the turbulent viscosity hypothesis for $\overline{u_{i}^{\prime} u_{j}^{\prime}}$ and the specification of the mixing length $l_{m}$.

The $k$-model does not allow for the transport of turbulent into regions where there is locally no generation. It is therefore inherently capable of simulating some phenomena more realistically than the mixing length model. However, the mixing length remains an empirical parameter and the knowledge about its value is almost absent for recirculating and three-dimensional flows. This model is found to be useful in boundary flows, where the mixing length is fairly well known.

\subsubsection{The two-equation model: the $k-\varepsilon$ model and mixed $k-\varepsilon$, Smagorinsky model}

To eliminate the shortcomings of the $k$-model, the length scale specification inherent in this model can be replaced by a transport equation for a turbulent quantity. The most extensively used quantity is the isotropic energy dissipation rate $\varepsilon$. This model belongs to the class of two-equation models, which are more efficient in the case of flows in a complex geometry and for higher Reynolds numbers. The two relevant macroscopic parameters in the $k-\varepsilon$ model are the turbulent mean kinetic energy and the mean energy dissipation. From dimensional analysis, one gets that the turbulent length scale: $l^{\prime} \sim \bar{k}^{3 / 2} / \bar{\varepsilon}$ and the turbulent velocity scale: $u^{\prime} \sim \bar{k}^{1 / 2}$. It follows that

$$
v_{\text {turb. }}=C_{\mu} \frac{\bar{k}^{2}}{\varepsilon} \quad \text { (Prandtl-Kolmorogov relationship) }
$$

where $k$ is turbulent kinetic energy, $\varepsilon$ is turbulence kinetic energy dissipation rate, and $C_{\mu}$ is an empirical constant. The turbulence viscosity couples the mean flow equations to the state variables of the turbulence model. The basic assumption of the $k-\varepsilon$ model is that vertical motions are mainly turbulent fluctuations and the mean component can be neglected. Due to the coarse horizontal resolution, it is further assumed that advective processes are insignificant compared to the local balance. The following transport equations must be solved to estimate $k$ and $\varepsilon$ respectively:

$$
\begin{aligned}
& \frac{\partial(u k)}{\partial x}+\frac{\partial(w k)}{\partial z}=\frac{\partial}{\partial z}\left(\frac{v_{t}}{\sigma_{k}} \frac{\partial k}{\partial z}\right)+P+G-\varepsilon \\
& \frac{\partial(u \varepsilon)}{\partial x}+\frac{\partial(w \varepsilon)}{\partial z}=\frac{\partial}{\partial z}\left(\frac{v_{t}}{\sigma_{\varepsilon}} \frac{\partial \varepsilon}{\partial z}\right)+C_{I \varepsilon} \frac{\varepsilon}{k}\left(P+C_{3 \varepsilon} G\right)-C_{2 \varepsilon} \frac{\varepsilon^{2}}{k}
\end{aligned}
$$


where the production terms by shear $P$ and by buoyancy $G$ are estimated, respectively,

$$
\begin{aligned}
& P=v_{t}\left[2\left(\frac{\partial u}{\partial x}\right)^{2}+2\left(\frac{\partial w}{\partial z}\right)^{2}+\left(\frac{\partial w}{\partial x}+\frac{\partial u}{\partial z}\right)^{2}\right] \\
& G=\beta g \frac{v_{t}}{\sigma_{d}} \frac{\partial \delta}{\partial z}
\end{aligned}
$$

Here $\beta$ is the volume expansion coefficient. The $k-\varepsilon$ model contains various empirical constants obtained from comprehensive data fitted for a wide range of turbulent flows. The following range of values from Rodi (1984) is most commonly used in environmental computations.

$$
C_{\mu}=0.09, \sigma_{k}=1.00, \sigma_{\varepsilon}=1.30, C_{1 \varepsilon}=1.44, C_{2 \varepsilon}=1.92
$$

Among these empirical constants, $C_{3 \varepsilon}$, in Equation (3.54), associated with the buoyancy term $G$ is quite uncertain (Choi and Garcia, 2002). In most applications, the above standard values of the parameters for the $k-\varepsilon$ model work well. However, for $C_{3 \varepsilon}$ in the buoyancy term, various alternative values have been proposed in literature. For shear flows, the buoyancy term should not affect the production of $G C_{3 \varepsilon}=0$ while the buoyancy term should contribute entirely to the production of $\mathrm{G}\left(C_{3 \varepsilon}=1\right)$ for vertical shear flows, as proposed by Choi and Garcia (2002). Svensson (1980) successfully stimulated the establishment of stably stratified mixed layers by the surface shear stress with $\left(C_{3 \varepsilon}=0.6\right)$.

An important aspect of closure modeling is to incorporate the effects of buoyancy. Although closure modelling has been a research area for more than 20 years, there is no universal modification for buoyancy to be applied to the existing closure models in all cases. When density gradients are present, the diffusion coefficients are damped. This means that entrainment and mixing will be overpredicted if the model does not account for this effect (MIKE3 Reference Manual, 2005). In the traditional Smagorinsky closure model, buoyancy effects are taken into account in an explicit manner following the empirical expression of Munk and Anderson (Munk and Anderson, 1948; Vested et al., 1998). In the $k-\varepsilon$ closure model, the Prandtl number $\sigma_{d}$, which appears in the transport equations for $k$ and $\varepsilon$, is modified explicitly by the expression

$$
\sigma_{d}=\left\{\frac{\left(1+\frac{10}{3} R i\right)^{3}}{1+10 R i}\right\}^{1 / 2}
$$

for stable stratification where $R i$ is the local gradient Richardson number 


$$
R i=-\frac{g}{\rho} \frac{\partial \rho}{\partial z}\left\{\left(\frac{\partial u}{\partial z}\right)^{2}+\left(\frac{\partial w}{\partial z}\right)^{2}\right\}^{-1}
$$

while $\sigma_{d}$ equals to unity for unstable stratification. The eddy viscosity is modified implicitly through the $k-\varepsilon$ equations and Kolmogorov-Prandtl relation.

The Smagorinsky formulation is the most popular model for the subgrid scale eddy viscosity proposed by Smagorinsky in 1963 (He et al., 2002). Here the eddy viscosity is linked to a filter size (grid spacing) and the large eddy strain rate, i.e. velocity gradients of the resolved flow field,

$$
\begin{aligned}
& v_{t}=l_{m}^{2} \sqrt{S_{i j} \cdot S_{j i}} \\
& S_{i j}=\frac{1}{2}\left(\frac{\partial u_{i}}{\partial x_{j}}+\frac{\partial u_{j}}{\partial x_{i}}\right)
\end{aligned}
$$

$l_{m}$ is a length scale which is replaced by the product $C_{s m} \cdot \Delta s$ where $\Delta s$ is the grid spacing and $C_{s m}$ is a constant indicating that the Smagorinsky formulation is a sub-grid scale turbulence closure model. The $C_{s m}$ values are used as calibration parameters. In most applications, vertical $C_{s m}$ value should be greater than the horizontal value. The higher vertical is to ensure a stronger dependency in the vertical direction rather than in the horizontal direction as the horizontal grid spacing often is much greater than the vertical grid spacing. The Smagorinsky formulation of the eddy viscosity is often selected as a compromise, between a constant value and the more sophisticated $k$ turbulence model and standard $k-\varepsilon$ turbulence model, which are much more time consuming due to the additional equation(s) to be solved at each time step.

\subsubsection{Limit of the turbulent-viscosity concept}

The concept of turbulent viscosity is properly applicable only when there is a significant separation between the length scale of non-homogeneity of the mean field and the mixing length of agitation. This condition is not satisfied in turbulent flows where the turbulent motions display a continuous distribution of scale sizes: turbulent motions do not occur at some characteristic mixing length. Furthermore, the largest spatial scales of turbulent motion are as large as the scale of non-homogeneity of the mean flow. Consequently, the concept of local transport does not have much justification except in a crude qualitative sense (Lévêque, 2005). 


\section{Chapter 4}

\section{PHYSICALLY-BASED NUMERICAL MODELLING}

\section{Summary}

This chapter presents descriptions of physical and bio-chemical processes in lakes and reservoir, the governing equations and their solution methods and the instantiation of models. As most of the governing equations for the models are hyperbolic partial differential equations, they are best solved using discretised numerical methods rather than analytically. Therefore, this chapter describes methods of time varying solutions, and how the governing equations are solved in spatially discretised grid systems. The modelling projects are accompanied inevitably by uncertainties, that is, affecting how the decision makers can trust the results of the modelling. It is therefore critical how the models are instantiated. State-of-the-art research trends in many cases are focusing on uncertainty analysis and quality assurance (QA). Therefore, a process of QA for modeling applications is introduced to conclude this chapter.

\subsection{Equations for Physical Process}

\subsubsection{Differential Equations}

Many physical processes of extreme importance to water engineers are governed by differential equations described hereafter (Popescu, 2004). Probably the best known of these processes are pollutant transport, conduction of heat and the propagation of waves. In describing such phenomena, we make use of ideas about gradients in space or time for particular parameters, which then lead to differential equations as models of the physical processes.

A differential equation is a relation that involves one or several derivatives of an unspecified function. The relation may also include the function itself. The order of the differential equation is the order of the derivative of the unknown function involved in the equation. An Ordinary Differential Equation (ODE) is a differential equation where the solution depends on one variable only. A typical ODE is the linear reservoir equation:

$$
\frac{d V}{d t}+k V=R(t)
$$

where $k$ (known) is the (positive) leakage constant, $R$ is the total recharge flux, $V$ is the volume of water stored in the reservoir, and $t$ is time. The solution $V$ depends on $t$ only. Therefore, Equation (4.1) is an Ordinary Differential Equation (ODE). The derivation 
symbol is $d$. Since an Euler's method solves only first order ordinary differential equations, it is problematic to solve a function of step size (both $\Delta x$ and $\Delta t$ ) and velocity which almost all of the physical processes have.

A Partial Differential Equation (PDEs) involves partial derivatives with respect to more than one independent variable. Independent variables typically include one or more space dimensions and possibly time dimension as well. The space variables will be denoted by $x$ and $y$, and the time variable, denoted by $t$, respectively.

The order of PDE is the order of the highest-order partial derivative appearing in the equation. The advection-diffusion equation that describes transport of a solute in water is a PDE:

$$
\frac{\partial c}{\partial t}+a \frac{\partial c}{\partial x}-D \frac{\partial^{2} c}{\partial x^{2}}=0
$$

where $c$ is the concentration of the dissolved species, $D$ is the diffusion coefficient and $a$ is the water velocity. This equation is typically used to model contaminant transport in rivers. It is a PDE because, as the $\partial$ symbol indicates, the solution $c$ depends on both space and time.

\subsubsection{Classification of Partial Differential Equations}

The general $2^{\text {nd }}$ order linear PDE for a function $u$ of two variables $x$ and $y$ has the form:

$$
a \frac{\partial^{2} u}{\partial x^{2}}+b \frac{\partial^{2} u}{\partial x \partial y}+c \frac{\partial^{2} u}{\partial y^{2}}=d \frac{\partial u}{\partial x}+e \frac{\partial u}{\partial y} f u+g
$$

where the coefficients $a . . . g$ may be functions of $x, y$, or both, or they may be constant. $u$ is the solution of the equation. The second-order PDE Equation (4.3) is classified according to the sign of its discriminant $\Delta$, given by: $\Delta=b^{2}-4 a c$. There are three classes of PDE.

- $b^{2}-4 a c<0$ : elliptic PDE, which tend to have smooth solutions, usually not too hard to solve by computation;

- $b^{2}-4 a c>0$ : hyperbolic, which can support solutions with discontinuities, can be difficult to solve computationally; and

- $b^{2}-4 a c=0$ : parabolic, which may have features of both.

A more general classification of PDEs (reflecting the physical phenomena they are describing) is that:

- Elliptic PDEs describe processes that have already reached steady state, and hence are time-independent (e.g. steady-state aquifer flow or heat diffusion). 
- Hyperbolic PDEs describe time-dependent, conservative physical processes, such as convection, that are not evolving towards steady state.

- Parabolic PDEs describe time-dependent, dissipative physical processes, such as diffusion, that are evolving towards steady state. (e.g. transient aquifer flow).

\subsection{Solution of Equations}

\subsubsection{Numerical Scheme}

Conceptual or physically-based models use differential relationships describing the changes in time of a set of variables. These formulations are either Ordinary Differential Equations (ODEs) or Partial Differential Equations (PDEs). As these ODEs and PDEs are very often complex and non-linear, it is not always possible to find analytical solutions to them. This is why numerical schemes are used to provide approximate solutions to the ODEs and PDEs.

Numerical schemes are techniques by which mathematical problems are formulated so that they can be solved using arithmetic operations. Numerical schemes involve large numbers of tedious calculations. These schemes have gained popularity due to the advancements in efficient computational tools such as digital computers and calculators. They are also used because sometimes experiments are impossible (e.g. weather forecast, avalanches, etc), and because numerical simulations are sometimes cheaper or faster.

The advantage of numerical schemes is that numerical answers can be obtained for problems that have no 'analytical' solution, because there are in general few analytical (closed-form) solutions for many practical engineering problems. Numerical schemes can handle large systems of equations, non-linearity, and complicated geometries that are common in engineering practice and that are often impossible to include in an analytical solution.

Generally, a mathematical model can be represented as a functional relationship of the form:

- Dependent variable $=f$ (independent variable, parameters, forcing function)

- Dependent variable =A characteristic that usually reflects the behavior or state of the system

- Independent variable = Are usually dimensions, such as time and space

- Parameters = Are reflective of system's properties or compositions

- Forcing Functions = Are external influences acting on the system

The basic principle of numerical schemes is discretisation of space and time. Discretisation means that the continuous domains of space and time are replaced with a set of selected points in space and time. The terms of the ODE or PDE are approximated 
using these points. Figure 4.1 provides an example where the independent variable, time, is discretised into a set of point $t^{1}, t^{2}, t^{3}, \ldots$. The approximation of the derivative of a variable $u$, is given by the relation:

$$
\frac{\Delta u}{\Delta t}=\frac{u\left(t^{2}\right)-u\left(t^{1}\right)}{t^{2}-t^{1}}=\frac{u^{2}-u^{1}}{t^{2}-t^{1}}
$$

in addition, can be viewed as a good approximation of $d u / d t$ between $t^{1}$ and $t^{2}$. Of course, the smaller the time interval $\Delta t=t^{1}-t^{2}$, the more accurate the approximation.

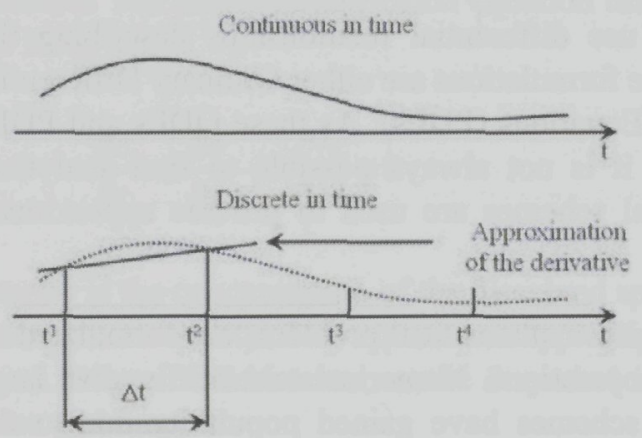

Figure 4.1. Principle of discretisation.

All mathematical models dependent on the types of numerical schemes used to translate the continuous derivatives, i.e., the terms in the Navier-Stokes equation, into a finite form appropriate for discretisation of computational time and grids. Other considerations include the choice of explicit or implicit time integration and the amount of artificial or numerical diffusion to include in the computation, which can directly affect the stability of the calculations.

\subsubsection{Explicit scheme for Hyperbolic PDEs}

The scheme where the value at the new time level can be computed directly from values at the previous time level is called an explicit scheme. The principle of the finite difference methods is the same for PDEs (partial differential equations) as for ODEs (ordinary differential equations). The only difference is that function has to be differentiated with respect to space in addition to derivation with respect to time. As an example, consider a linear advection equation, which is one of the simplest possible PDE:

$$
\frac{\partial u}{\partial t}+a \frac{\partial u}{\partial x}=0
$$

where $u$ is assumed positive. The word 'linear' implies that the equation consists of a sum of separate terms and the advected quantity, $u$, only appears once in each term. 
When advection velocity $a$ is constant, the general solution to Equation (4.5) is:

$$
u(x, t)=f(x-a t)
$$

where $f(s)$ is an arbitrary single-valued differentiable function. The easiest way to prove this is to substitute Equation (4.6) into the left-hand-side of Equation (4.5). If $s=x$-at then

$$
\frac{\partial f(s)}{\partial t}+a \frac{\partial f(s)}{\partial x}=\frac{d f}{d s} \frac{\partial s}{\partial t}+a \frac{d f}{d s} \frac{\partial s}{\partial x}=\frac{d f}{d s}\left(\frac{\partial s}{\partial t}+a \frac{\partial s}{\partial x}\right)=\frac{d f}{d s}(-a+a \cdot 1)=0
$$

In other words, whatever $u$ represents will be carried along at a constant velocity $a$. Its shape will be preserved.

Returning now to Equation (4.5) and trying to solve this equation using the discrete approach, space has to be discretised in addition to time. In addition to the discrete set of points along the $t$-axis, a set of discrete points along the $x$-axis is set up as Figure 4.2.

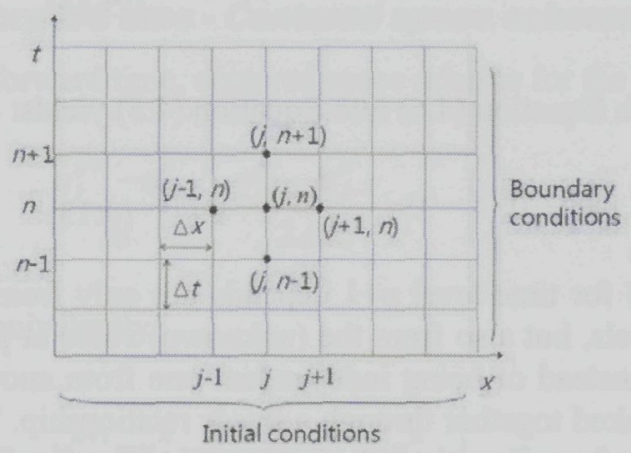

Figure 4.2. Discretisation of time and space.

Each of the derivatives (with respect to time and space) in Equation (4.5) is approximated using this set. One way of approximating the time-derivative between points $(j, n)$ and $(j, n+1)$ is:

$$
\frac{\partial u}{\partial t} \cong \frac{u_{j}^{n+1}-u_{j}^{n}}{\Delta t}
$$

while the space-derivative can be approximated using the following relationship:

$$
\frac{\partial u}{\partial x} \cong \frac{u_{j}^{n}-u_{j-1}^{n}}{\Delta x}
$$

where $\Delta x$ is the distance between points $j-1$ and $j$. Putting these two relationships together into Equation (4.5) yields:

$$
u_{j}^{n+1}=C r_{j}^{n} u_{j-1}^{n}+\left(1-C r_{j}^{n}\right) u_{j}^{n}
$$




$$
C r_{j}^{n}=\frac{a \Delta t}{\Delta x}
$$

where $\mathrm{Cr}$ is called the Courant number. Assuming that the values for $u$ are known at any point $j$ at time level $n$, the value for $u$ at any point at time level $n+1$ can be computed using Equation (4.10). Equation (4.10) cannot be applied at the left-hand boundary, where $j=1$, because there is no point to the left of the left boundary. Additional information must be provided: the boundary condition $u_{1}^{n=1}$.

\subsubsection{Implicit Schemes for Hyperbolic PDEs}

The space derivative is estimated using the values at time level $n+1$ :

$$
\frac{\partial u}{\partial x} \cong \frac{u_{j}^{n+1}-u_{j-1}^{n+1}}{\Delta x}
$$

Substituting this expression together with Equation (4.6) into Equation (4.5) yields:

$$
-C r^{n+1} u_{j-1}^{n+1}+\left(1+C r^{n+1}\right) u_{j}^{n+1}-u_{j}^{n}=0
$$

Then, the (unknown) solution at point $j$ for time level $n+1$ depends not only from the (known) values at the previous time levels, but also from the (unknown) value at point $j-1$ at time level $n+1$. In other words, instead of being independent one from another, the values for $u$ at time level $n+1$ are linked together through a linear relationship. This means that a system of equations of the form Equation (4.13) must be solved: $u_{1}{ }^{n+1}$ is known because it is the upstream boundary condition, and $u_{2}{ }^{n}$ is known from the previous time step. Then it is possible to compute $u_{2}^{n+1}$. Sweeping along $j$ in ascending order provides $u_{3}^{n+1}, u_{4}^{n+1}$ etc.

A scheme where there is a relationship between several points at the unknown time level, leads to a system of equations. The equations of this system must be solved simultaneously to compute the unknowns. Such a scheme is called an implicit scheme. If the relationship between the unknown points is complex (for instance, non-linear), the system must be solved in an iterative way.

\subsection{Methods of Time Variable Solutions}

\subsubsection{Discretisation}

An adequate treatment of unstable advection terms is a matter of utmost importance for the majority of applications of numerical hydrodynamic model. When solving transport equations (advection terms) using numerical methods, the equations are solved in a 
finite number of discrete points within the computational domain named computational mesh or grid. There are different strategies to convert the original continuous differential equations to the discrete finite differences equations. These are usually called the discretisation schemes. There are two kinds of discretisation scheme: temporal and spatial. The spatial discretisation is described in the section of reservoir modelling.

\subsubsection{Standard Discretisation Techniques}

There are various approaches to time variable differencing equations, and for each approach, they will be occupied to investigate:

- its stability

- its accuracy

- its suitability for a particular problem.

\section{1) Forward time - Centered space scheme (FTCS)}

The forward time, centered space scheme for the advection equation is:

$$
\frac{u_{j}^{n+1}-u_{j}^{n}}{\Delta t}+a \frac{u_{j+1}^{n}-u_{j-1}^{n}}{2 \Delta x}=0
$$

or, equivalently:

$$
u_{j}^{n+1}=u_{j}^{n}-\frac{1}{2} \frac{a \Delta t}{\Delta x}\left(u_{j+1}^{n}-u_{j-1}^{n}\right)
$$

The Fourier analysis of these schemes will show that they are unconditionally unstable. However, as a rule, numerical models use a FTCS scheme to calculate the very first time-step despite the fact that a FTCS scheme is unconditionally unstable. It is usually assumed that the errors introduced by using the FTCS scheme once are negligible when compared with all the other errors that are present in the model. In the same way FTBS (forward time, backward space), BTBS (backward time, backward space), ....(etc) schemes can be defined. The schemes of backward space are also called upwind schemes because the information that is used in space comes from upstream.

\section{2) Centered and Upwind Schemes}

The most interesting scheme of this kind of schemes is the CTCS (Centered time, centered space scheme):

$$
\frac{u_{j}^{n+1}-u_{j}^{n-1}}{2 \Delta t}+a \frac{u_{j+1}^{n}-u_{j-1}^{n}}{2 \Delta x}=0
$$


Solving for the value at time $(n+1) \Delta t$ gives

$$
u_{j}^{n+1}=u_{j}^{n-1}-\frac{a \Delta t}{\Delta x}\left(u_{j+1}^{n}-u_{j-1}^{n}\right)
$$

The value at time $(n+1) \Delta t$ is obtained by adding a term to the value at $(n-1) \Delta t$. The ratio $C r \equiv a \wedge t / \Delta x$ will be found to be crucial. Using the Courant Number,

$$
u_{j}^{n+1}=u_{j}^{n-1}-\operatorname{Cr}\left(u_{j+1}^{n}-u_{j-1}^{n}\right)
$$

Assuming that we know the solution up to time $n \Delta t$, the values at time $(n+1) \Delta t$ can be calculated, and the solution advanced by one time-step in this way. Then the whole procedure can be repeated to advance the solution to $(n+2) \Delta t$, and so on.

\subsubsection{High-Resolution Scheme}

An analysis of numerical diffusion in models for lakes should start by looking into the way a model deals with advection (Wang and Hutter, 2003). To achieve a satisfactory numerical modeling of advection is always a dilemma to the researchers in the field of computational fluid dynamics. Solutions produced by standard discretisation techniques are typically corrupted by nonphysical oscillations and/or excessive numerical diffusion.

The second-order methods or other higher-order methods give high-order accuracy, but at the same time, nonphysical oscillatory behavior in regions where steep gradients exist or may be generated. The central difference scheme (CDS) is one of the examples. The standard centred advection scheme can lead to numerical oscillations, but it is still used in various models. This scheme may lead to unrealistic oscillatory behavior in an implicit solution or to non-convergent solutions in an explicit computation in regions where advection strongly dominates diffusion.

On the other hand, when using the classical first-order upwind difference scheme (UDS), the oscillations may be replaced by an unacceptable global artificial diffusion. In 1979, Leonard proposed the quadratic upstream interpolation for convective kinematics scheme (QUICK) for quasi-steady flow situations. The methods have the desirable simultaneous properties of high accuracy, inherent numerical advective stability, algorithmic simplicity and a physically appealing foundation. Recent ocean models use the third-order upwind-weighted schemes, such as ELCOM (Estuary and Lake Computer Model developed by Hodges, et al., 2000) based on the implementation of Leonard's QUICK and QUICKEST (QUICK. with Estimated Streaming Terms) scheme. Although these third-order schemes provide superior accuracy, they are not typically monotonic or positive definite. They still do contain visible implicit numerical dispersion and diffusion. So far, the study of accurate modelling of advection focuses mainly on scalar transports such as temperature and/or a tracer. 
Modern high-resolution schemes are based on flux/slope limiters, which switch between linear high- and low-order discretisations adaptively, depending on the smoothness of the solution (Kuzmin and Turek, 2004). The idea of flux limiters is to limit the flux of $q$ between grids and subsequently to limit spurious growth in the grid averages near discontinuities. Hence, the corrective flux is multiplied by a limiter. The limiter is normally computed as the ratio between two solution differences, taken at two stations in the upwind direction. When this ratio becomes negative, a local extremum has been encountered, and the limiter vanishes. Example of popular limiters is the Minmod (most diffusive) limiter (Juntasaro and Marquis, 2004), which is always a safe choice, and the Superbee (least diffusive) limiter (Yee, 1989), which is said to be over-compressive, i.e. it tends to sharpen gradients (Fjelde and Karlsen, 2002) and is therefore less accurate for smooth solutions (Zalesak, 1987).

According to Wang and Hutter (2003), comparison of a series of numerical schemes including a third order QUICK scheme and the high resolution FCT (Flux-Corrected Transport) technique (Boris and. Book, 1973) with respect to dimensional advectivelydominated problems shows that the second-order TVD (Total Variation Diminishing method, which is a compromise between the two schemes) (Carofano, 1984; Yee, 1989) is most preferable. The influence of the three different advection schemes: the most frequently used traditional centred and upwind difference schemes (CDS and UDS) as well as the high resolution TVD method have been investigated for an ocean/lake model.
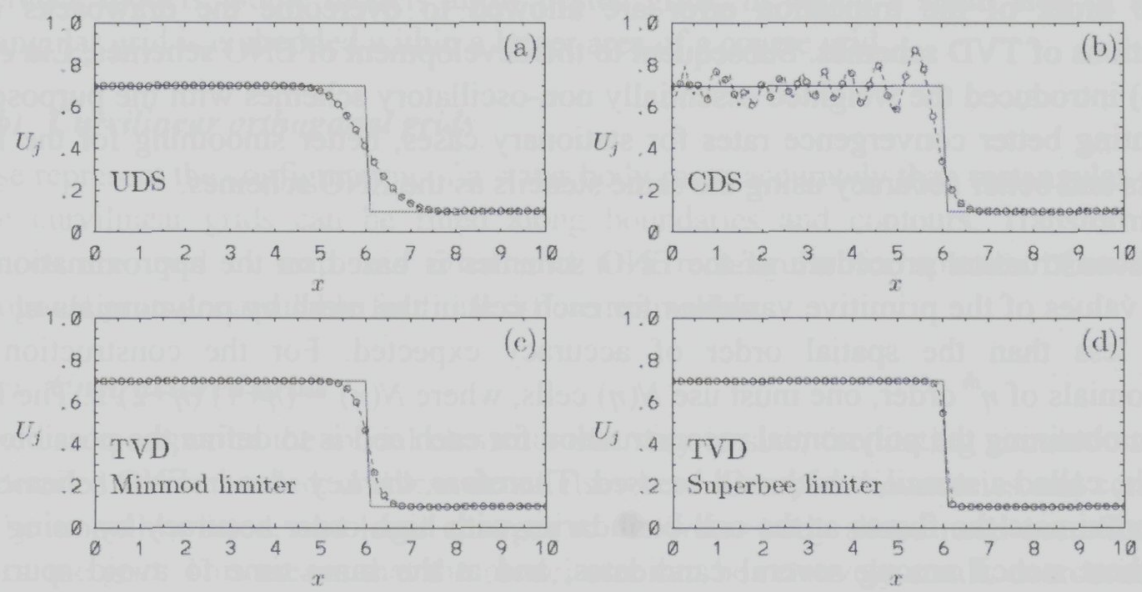

Figure 4.3. Comparison on different numerical methods for one-dimensional linear advective problem with discontinuous initial data (from Wang \& Hutter, 2003).

The central idea of TVD method is, on the one hand, to avoid the introduction of underand overshoots (numerical oscillation), and on the other hand, to maintain numerical diffusion as small as possible. The TVD method is demonstrated to be the most suitable scheme to handle advection terms emerging in lake dynamic equations for both homogeneous and stratified cases (Wang, 2003). In summary, the following difference schemes are applied: 


$$
\begin{aligned}
& \frac{\varphi^{t+\Delta t}-\varphi^{t-\Delta t}}{2 \Delta t}+\underbrace{\left(u \frac{\partial \varphi}{\partial x}\right)^{t}+\left(v \frac{\partial \varphi}{\partial y}\right)^{t}+\left(w \frac{\partial \varphi}{\partial z}\right)^{t}}_{\text {TVD }} \\
& =\underbrace{\left(\frac{\partial}{\partial x}\left(K_{\varphi}^{h} \frac{\partial \varphi}{\partial x}\right) t-\Delta t\right)+\left(\frac{\partial}{\partial y}\left(K_{\varphi}^{h} \frac{\partial \varphi}{\partial y}\right) t-\Delta t\right)+\left(\frac{\partial}{\partial z}\left(K_{\varphi}^{h} \frac{\partial \varphi}{\partial z}\right) t-\Delta t\right)}_{\operatorname{CDS}}+R_{\varphi}^{t}
\end{aligned}
$$

where superscripts, $t-\Delta t, t$, and $t+\Delta t$ indicate the three consecutive time levels, while the notations under the terms denote the used spatial discretisation schemes.

However, both the theory and the algorithms of TVD are essentially one-dimensional and no rigorous generalization to the multidimensional case is available to date. As a matter of fact, most of the CFD (Computational Fluid Dynamics) codes utilizing a (quasi-) TVD discretisation of convective terms resort to directional splitting on Cartesian grids (Kuzmin et al., 2004).

Besides this fact, it is well known that TVD schemes have their order of accuracy reduced to first order in the presence of shocks due to the effect of the limiters (Wolf and Azevedo, 2005). This observation initiated the development of essentially nonoscillatory (ENO) schemes introduced by Harten et al. (1987) in which oscillations up to the order of the truncation error are allowed to overcome the drawbacks and limitations of TVD schemes. Subsequent to the development of ENO schemes, Liu et al. (1994) introduced the weighted essentially non-oscillatory schemes with the purpose of presenting better convergence rates for stationary cases, better smoothing for the flux vectors and better accuracy using the same stencils as the ENO schemes.

The reconstruction procedure of the ENO schemes is based on the approximation of mean values of the primitive variables for each cell in the mesh by polynomials of one order less than the spatial order of accuracy expected. For the construction of polynomials of $\eta^{t h}$ order, one must use $N(\eta)$ cells, where $N(\eta)=(\eta+1)(\eta+2) / 2$. The first step in obtaining the polynomial reconstruction for each cell is to define the possible set of cells, called a stencil, which will be used. Therefore, the key idea in ENO schemes is to approximate the fluxes at the cell boundaries with high order accuracy by using the smoothest stencil among several candidates, and at the same time to avoid spurious oscillations near shocks and discontinuities (Wu, 2006).

Differently from the ENO schemes, the weighted essentially non-oscillatory (WENO) schemes use all the calculated polynomials. These polynomials are added together using weights, which are computed for each one of the polynomials as being proportional to its respective oscillation indicator. The main idea in the WENO reconstruction is to attribute the computed weights for each polynomial with the aim of reconstructing a new polynomial (Wolf and Azevedo, 2005). 


\subsection{Lake/Reservoir Modelling}

\subsubsection{Grid Generation and Numerical Solution}

\section{1) Grid generation}

The first step in a physically-based numerical modelling is to discretise the solution domain (the water body) in one, two or three dimensions representing the system as a one-, two-, or three- dimensional network. The next step is to define the quantities to be modelled (water levels, currents, constituent levels etc) across this numerical grid. The grid dimension and the representation of the model quantities significantly affect the level of detail available from a model, the quantity of data required to calibrate the model, the time taken to set it up, and the computational requirements (Moffatt \& Nichol Engineers, 2005). The different types of grids have the following characteristics:

\section{a) Rectangular grids}

These suffer from the significant drawback that the boundaries of channels and other water bodies are represented as stair-step edges, requiring a fine grid to represent the edges in detail. Increasing the resolution of the grid for a small region to account for narrow channels or structures may require changes to the spacing throughout the model domain. This can more than offset the relatively high computational efficiency of finite difference models. Some models allow nested grids, in which a small area of a fine rectangular grid is embedded within a larger area of a coarse grid.

\section{b) Curvilinear orthogonal grids}

These represent the configuration of a water body more accurately than rectangular grids, since curvilinear grids can be fitted along boundaries and contours. Transformation relations are used to map this fitted grid to a rectangular for solution. Curvilinear orthogonal grids are available for finite difference models.

\section{c) Unstructured grids}

These can incorporate the model elements that vary in size, facilitating simulations that are detailed where needed, and less detail elsewhere. Complex boundaries and contours can be smoothly fitted, and local changes to the water body shape need not affect the rest of the grid. This means that the model size can be relatively small, decreasing the computational requirements. However, unstructured grids can be very time consuming to set up, and the pre-processing tools used to construct the model become critical.

\section{d) Staggered Grids}

These are used for the velocity components. The finite volume method starts, as always, with the discretisation of the flow domain and of the relevant transport equations: $\mathrm{x}, \mathrm{y}-$ momentum and continuity equations. It seems logical to define these at the same locations as the scalar variables such as pressure, temperature etc. However, if the velocities and pressures are both defined at the nodes of an ordinary control volume a 
highly non-uniform pressure field can act like a uniform field in the discretised momentum equations. It is clear that, if the velocities are defined at the scalar grid nodes, the influence of pressure is not properly represented in the discretised momentum equations. A remedy for this problem is to use a staggered grid for the velocity grid. The idea is to evaluate scalar variables, such as pressure, density, temperature etc., at ordinary nodal points but to calculate velocity components on staggered grids centred around the cell faces. Hence, no interpolation is needed to calculate velocities at the scalar cell faces (Versteeg and Malalasekera, 1995).

\section{2) Numerical Solutions}

There are three popular approaches to implementing the numerical solution of the partial differential equations:

a) Finite difference method represents the water levels at a set of discrete points for each time step. The typical solution grid is a network of straight or curved lines with nodes at the intersections. Currents are normally described at the links between the nodes.

b) Finite element method divides the solution domain (the water body) into a set of triangular or polygonal elements. The water levels and currents are described as linear or quadratic functions across each element.

c) Finite volume method is such that the known value at a given time level is no longer the value of variable at a point, but the average value over a domain (overall cell).

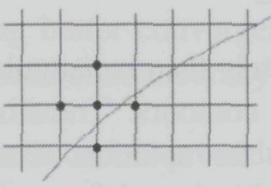

a) A finite difference grid

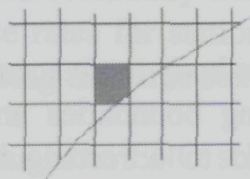

b) A finite volume grid

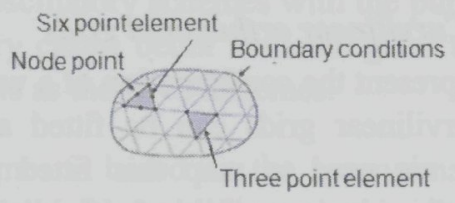

c) A finite element grid

Figure 4.4. Principle of discretisation.

By comparison with certain FDMs, FVMs ensure conservation of the total quantity contained in the computational domain. Therefore, "Finite volume" refers to the small volume surrounding each node point on a mesh. FVM are thus always conservative, i.e. no mass is lost artificially. Moreover, finite volume methods deal with discontinuous profiles more easily than finite differences. Finite volume methods only depend on estimates of fluxes between cell interfaces. The finite difference approach is based upon the (discrete) estimate of the derivatives, thus, it is based upon the assumption of continuity of the variable over the domain of computation. The ability of the finite volume approach to deal with discontinuous flow makes it possible to solve problems involving shocks, such as hydraulic jumps, wave breaking or dam break flood waves, accurately. 
Time is advanced forward and solved explicitly in all three methods. Finite difference calculations are more computationally efficient for a given network size than finite element calculations. However, this is often offset by the fact that finite difference models are generally limited to rectangular or curved grid, while finite element grids are better able to accommodate irregularly shaped model domains. This means that finite difference solutions often require finer grids than finite element solutions to model complex shapes. The time step for finite element solutions can also be longer, which may speed up the simulation.

In summary, while the choice of finite difference versus finite element models is important, the decision as to model type will probably be made on the bases of shape and size of the model domain, length of simulation, coupling requirements with other models. For one-dimensional models, the choice of network type is not an issue and the selection of one-dimensional model is made on other grounds such as numerical stability.

As the model dimension increases from one to two or three dimensions, the level of detail, the data requirements, and the time required to set up and modify the model, and the data requirements all increase exponentially. In some modelling studies, the use of a combination of models would be appropriate. This will involve a mix of dimensions, with channels or control structures modelled as one-dimensional models, and limited areas where stratification is important in three-dimensional models. This kind of modelling allows a one-dimensional elements to be incorporated within a three dimensional model, though the feasibility of this kind of modelling varies significantly from model to model, and must be specified in detail.

The Courant number, which is a fundamental concept in the numerical solution of hyperbolic partial differential equations, is the ratio of physical wave celerity $c=d Q / d A$ or $\sqrt{g h}$ to grid celerity $\Delta x / \Delta t$. therefore the courant number, $C r$ is defined as follows:

$$
C r=c \frac{\Delta t}{\Delta x}
$$

where, $\Delta t$ is the time step, $\Delta x$ is the grid spacing in one of the horizontal directions. As the barotropic information (about surface elevations and velocities) in the computational grid travels at a speed corresponding to the celerity, the Courant number actually expresses the information about how many grid points a surface element or fluid particle moves in one time step.

When advection dominates dispersion, designing a model with a small $(<1)$ Courant number will decrease oscillations, improve accuracy and decrease numerical dispersion.

\subsubsection{Coupling Hydraulics and Transport}


To simulate chemical transport, the velocity field represented by $u$ in the transport equation must also be computed. The model that calculates $u$ is called the hydrodynamic or hydraulic model. Thus, to simulate transport, the hydrodynamic and transport models must be properly coupled.

Whether the hydrodynamic and transport models must be implicitly coupled or whether they can be run in series depends on the importance of buoyancy effects. If the system is free from buoyancy effects, the hydrodynamics are independent of the transport; hence, they can be run first and their output stored. Then, many transport simulations can be run using the hydrodynamic data without re-running the hydrodynamic code. If buoyancy effects are present in the water body, then the transport of buoyancy (heat, salinity, or both) must be coupled with the hydrodynamics, and both models must be run together. Once the output from the coupled model is stored, further transport simulations can be run for constituents that do not influence the buoyancy (these are called passive constituents). Because the hydrodynamic portion of the model is computationally expensive, the goal in transport modeling is to de-couple the two models as much as possible.

\subsubsection{Long Narrow Reservoir Model}

There are some distinct advantages in modeling a long narrow reservoir. For example, the horizontal length scale along the reservoir is much greater than vertical and width length scales, so the Coriolis acceleration forces are negligible (Cushman-Roisin, 1994; de la Fuente and Niño, 2008). Reservoir stratification normally requires a threedimensional model, particularly where inflows become density currents at a depth in the reservoir where the water density is the same. A long narrow reservoir having a linear channel flow can abbreviate the laterally horizontal flow, $v_{y}$, with an averaged velocity, $\bar{u}_{x}$. One can call this model a "one plus one-dimensional model". In contrast, a twodimensional model generally considers the lateral circulation in large water-bodies such as lakes or estuaries. The flow characteristics in a two-dimensional model are normally assumed uniform down through the water column at each computation point, so that stratification is not described. However, the one plus one-dimensional models use one horizontal and one vertical dimension, rather than two horizontal dimensions. This allows the vertical structure of the hydrodynamics in a narrow channel to be evaluated. These kinds of models reduce the large computational and data requirements of full three-dimensional models. Such models are particularly useful for deep and long narrow reservoirs.

\subsubsection{Using Ocean Models for Lakes/Reservoirs}

Most three-dimensional models used in physical limnology have been designed mainly for the simulation of ocean and/or estuary circulation. This is because physical limnology deals with methods similar to oceanography, except that it largely 
concentrates on the responses of closed basins to applied forces and exchanges of heat between inflows and receiving water.

Although the overall circulation of large lakes is characterized by complicated variations in space and time, due to the fast advance in computational fluid dynamics, there is a wide range of three-dimensional hydrodynamic models applying finite difference methods (FDM), Finite Element Methods (FEM), and Finite Volume Methods (FVM), and applying combinations of them (Umlauf et al., 1999).

Whatever numerical method is applied in the model, most oceanographic models are solved on an explicit scheme both in the horizontal and vertical dimensions. For finite difference methods, the Alternating Direction Implicit (ADI) method, which is a finite difference method for solving parabolic and elliptic partial differential equations, has found wide applications in most models. Many of these models have had successful applications in oceanic and estuarial applications but very few in lakes/reservoir applications (Umlauf et al., 1999).

Wang and Hutter (2003) suggest that the failure of oceanographic models to depict lake physics adequately is due to their failure to depict the internal waves, as stipulated by Hodges et al. (2000). The internal waves are not depicted because they are overly damped owing to the large explicit or implicit numerical diffusion that is built into the numerical codes to stabilize them, deduces Wang and Hutter (2003).

In oceans, this problem is not experienced; the reason is based on the kinds of motion experienced in both scenarios. Accurate modelling of advection in momentum equations may be less important in ocean simulations since the flow is largely geo-strophic, and hence advection does not dominate the dynamics.

For example, if the momentum equations in the RANS system are written in form of advection-dispersion equations:

$$
\begin{aligned}
& \frac{\partial \varphi}{\partial t}+u \frac{\partial \varphi}{\partial x}+v \frac{\partial \varphi}{\partial x}+w \frac{\partial \varphi}{\partial x} \\
& =\frac{\partial}{\partial x}\left(K_{\varphi}^{h} \frac{\partial \varphi}{\partial x}\right)+\frac{\partial}{\partial y}\left(K_{\varphi}^{h} \frac{\partial \varphi}{\partial y}\right)+\frac{\partial}{\partial z}\left(K_{\varphi}^{v} \frac{\partial \varphi}{\partial z}\right)+R_{\varphi}
\end{aligned}
$$

where $\phi$ may be either a velocity component $u$ or $v$, or the temperature $T$, and $K_{\phi}^{h}$ is the horizontal momentum or thermal diffusivities. $R_{\mathscr{b}}$ stands for sink or source terms, for momentum could be Coriolis force and pressure gradient. In oceans compared to lakes, the Coriolis forces and pressure gradients are very large, and therefore dominate the motions on the left hand side of the equation, while in lakes, these two terms are negligible, but instead are replaced with wind and river inflows that depend on the advection coefficients to distribute their energy through the water body. Hence, in lake dynamics, as can be seen from the equation, the velocity gradients especially near the 
thermocline and the water surface dominate the hydrodynamics. Therefore, in such cases, a reasonable simulation of the advection terms in the momentum equations is of same importance as for temperature and tracer transport problems.

Moreover, as mentioned by Umlauf et al. (1999), modelling lakes or reservoirs using oceanographic models has its limitations, and in some cases, the ocean models have totally failed to capture the physics of the lakes. The main reason is due to the small length scales of motion in a lake compared to an ocean. This difference escalated into highlighting more problems experienced in lake or reservoir models:

- The explicit scheme is influenced by the horizontal diffusion terms, which in turn affect the stability of the model. Diffusion in lakes is best solved with an implicit scheme but this would be very computationally expensive

- Wang and Hutter in their works on the ADI method have shown that when the ADI technique is applied in an explicit scheme, this does not suffice with lakes (Umlauf et al., 1999). They say the ADI technique introduces some instability in the models.

However, one-dimensional vertical models or laterally averaged two-dimensional models (like CE-QUAL-W2) have found successful application in reservoirs and lakes environmental modelling compared to three-dimensional models. The main reason could be because they are designed specifically for reservoirs and lakes, but some authors claim, their success is attributed to the ability of these models to capture accurately the large scale physics of the reservoir (though they hardly depict the small scale motions). Secondly, these models are very efficient in their use of computational memory and time

\subsubsection{Unresolved Effects of Lake/Reservoir Numerical Modelling}

The problems with lake models do not stop with the turbulence modelling; there are still other processes that are still uncertain in their modelling.

\section{1) Wind and surface thermo-hydrodynamics}

The primary difficulty with accurately modelling the surface effects is usually a lack of accurate environmental data rather than any fundamental inadequacy of existing models. (Hodges et al., 2000). The rate of turbulent kinetic energy production associated with wind-induced shear (see Figure 4.5) is defined for a constant wind speed. However, the wind speed seldom remains constant over periods equal to typical time steps used in numerical reservoir simulations, i.e., a few hours to 1 day.

Ignoring the variability of the wind speed over the time step of the numerical simulation may cause an underestimation of the actually available energy for mixing. The amount of energy used for generating waves in the case of frequently interrupted winds is larger than in the case of constant winds because the surface waves are dissipated when there is a pause in the wind action. Additional energy is required after such a pause for building up the waves to the level they were before the pause in the wind action. The 
variability of the wind pattern may be seasonal or may be caused by general weather conditions in the area.

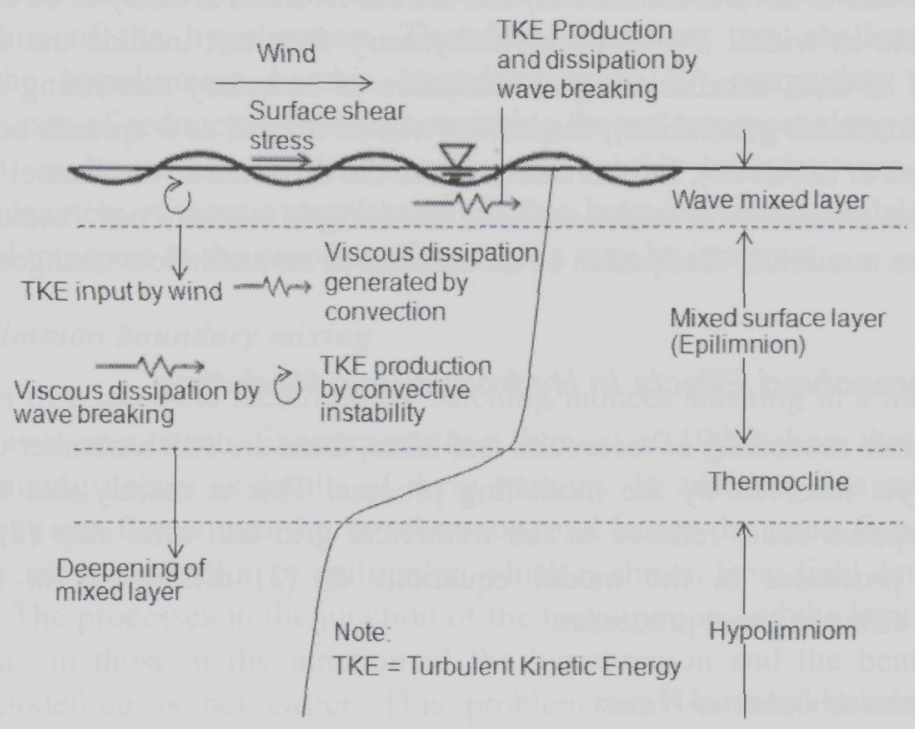

Figure 4.5. Definition of Physical Processes Affecting Growth of WindMixed Surface Layer (Source: Findikakis and Law, 1999).

Another characteristic of the wind pattern is the variability of wind direction. Short duration, interrupted winds characterized by frequent changes in direction are not as effective as constant direction, uninterrupted winds in contributing to the growth of the mixed layer. Changes in wind direction are again more frequent during summer and early fall than in late fall and winter (Findikakis and Law, 1999).

In particular, the wind over the entire surface of most lakes is influenced by the surrounding topography such that local wind blocking may give rise to consistent wind spatial gradients that directly drive surface gyres. As multiple wind stations cannot be practically maintained around most lakes, a major challenge to $3 \mathrm{D}$ hydrodynamic lake modelling is the prediction of spatially varying wind fields.

\section{2) Dense underflow Events}

Reservoirs formed from flooded river valleys or natural lakes in geographical rifts may experience dense inflows, moving down the channel bottom for tens of kilometres before plunging through the metalimnion. Such flows may take several days from initiation in the catchment to insertion in the hypolimnion. Capturing the speed of propagation, entrainment rate and depth of underflow are critical to modelling its correct insertion in the lake density structure. Modelling these inflows is critical to catchment management decisions. 
The complex morphologies of reservoirs present a modelling challenge because a dense inflow will seek the lowest path, that is, the old river-bed. While a reservoir may have a wide width of one to several kilometers, and the old riverbed is likely to be tens of a few hundred meters in width. If the model bathymetry does not include the old riverbed (through lack of data, insufficient grid resolution or boundary smoothing required for curvilinear coordinate generation), the inflow will be diluted as it spreads across a wide region (Hodges et al., 2000). Furthermore, where the drowned river channel is known, it is likely to be sinuous and under-resolved at the grid scale. Poor resolution in the channel causes numerical dissipation of momentum as an underflow changes from $x$ to $y$ directions.

\section{3) Other Unresolved Effects in Hydrodynamic Modelling}

In hydrodynamic modelling of reservoirs and lakes, there are still a number of processes that are not yet resolved by the modelling process. This is mainly due to: (1) their temporal or spatial scale relative to the numerical grid and time step (2) neglect of fundamental processes in the model equations or (3) difficulties in numerically capturing the effect of these processes.

\section{a) Short-period Internal Waves}

Although their energy content is smaller than basin-scale internal waves, internal waves are presumed to be an important trigger for internal turbulence and as a mechanism for energy and momentum transport to the lake boundary. The process by which basin scale internal waves evolve into trains of smaller scale internal waves is fundamentally non-hydrostatic, and they occur at length scales of a very fine sub-grid. So the hydrostatic assumption becomes problematic when dealing with internal waves. The hydrostatic assumption introduces a new form of numerical diffusion that steepens out the internal waves to break prematurely. In addition, this also introduces a numerical dissipation of momentum accompanied by a numerical diffusion of density structure, that is, a numerical trail of mixing; the model becomes more dependent upon numerical diffusion than the physics of the phenomena.

Non-hydrostatic engines would avoid this problem but cannot be resolved also, to the sub-grid scales of the internal waves. Then with a high discretisation of the grid, numerical diffusion may be alleviated but the model might still be dominated by the truncation error. So, in short, it follows that the non-hydrostatic internal waves are a closure problem for geophysical numerical models. Their processes are not resolved by governing equations and their effects are dependent on processes that are fundamentally sub-grid scale: this is a basic challenge that has not been addressed in any existing turbulence closure model (Hodges et al., 2000).

\section{b) Hypolimnetic benthic boundary layer}

From the perspective of the hydrodynamic model, the boundary layer provides a drag that slowly dissipates the large-scale internal wave motions. This implies that the hypolimnion is of secondary importance in modelling the basin scale internal wave 
dynamics but not in terms of the transport processes of biogeochemical variables. So the interaction of the benthic boundary layer with the mass transport due to Kelvin waves may be a critical component in the transport into the metalimnion of nutrients deposited in the benthos of the hypolimnion. Therefore, there are two challenges faced in modelling the hypolimnion benthic boundary layer: (1) computing the effective resuspension rate of sediments, and (2) computing the net transport along the boundary. So, in short, in basin scale hydrodynamics the benthic boundary net flux can be modelled adequately without a supplicated benthic boundary layer model, but the biogeo-chemical transport in the near-boundary region may be incorrect.

\section{c) Metalimnion boundary mixing}

Along slopes of a lake, the metalimnetic seiching induces sloshing in a manner similar to surface waves on a beach. Experiments (e.g. Ivey et al, 1999) show that a significant amount of mixing occurs as results of these motions. In addition, this mixing plays a role in the nutrient fluxes into the metalimnion, and hence the mass flux between the hypolimnion and metalimnion or epilimnion which is shown from field data (Ostrovsky et $\mathrm{al}, 1996)$. The processes in the junction of the metalimnion and the benthos are more energetic than in those at the junction of the hypolimnion and the benthos, but the numerical modelling is not easier. This problem can be reduced when boundary conforming grids (like the $\sigma$-method) are used, instead of a Cartesian mesh. Although, whichever grid method is used, the fundamental problem of the excessive computational cost of a fine grid required resolving the small-scale processes along the boundary remains (Hodges et al, 2000).

Consequently, using the numerical techniques and computational power that are presently available, the fundamental basin-scale motions can be modelled with reasonable accuracy in three dimensions over weekly to monthly time scales. To sum up, accurate simulation of the thermal structure rests on resolution of two numerical issues: (1) control of numerical diffusion, and (2) modelling of unresolved turbulent processes that lead to mixing.

\subsubsection{Integration of Environmental Models}

There are five categories of models (see Figure 4.6) applied in environmental studies, each designed for a specific portion of the environment. These include hydrodynamic/hydraulic, surface water quality, hydrology/watershed, ecological and ground water models, each describing a specific portion of the hydrologic cycle (Limno-Tech Inc., 2002).

The environmental models generally address one or more system processes, such as hydrodynamics, sediment transport, water quality, and/or ecological effects. Therefore, there is no single model that can quantify the ecological impacts of a particular watershed management scenario throughout the watershed including the reservoir. However, a suite of linked models can be used to address these types of management 
questions for different management scenarios. Figure 4.6 represents the five categories of models and their potential interconnectivity (Limno-Tech Inc., 2002).

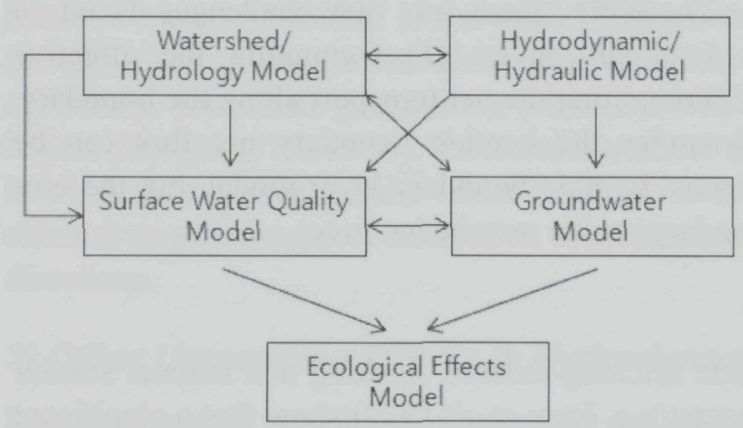

Figure 4.6. Different combinations catchments.

The linking or integration of different models depends on the particular management scenario being studied. This kind of integration can provide very useful model framework for assessment in any decision support system framework.

\subsection{Instantiating Models}

\subsubsection{Calibration}

In modeling, it is common for modelers to calibrate a model to a set of observed data, visually compare some time-series plots of predictions and observations, declare the model to be 'reasonable,' and proceed to use the model for inference or decision support (Stow et al., 2003).

Calibration is the process of modifying the input parameters to a model until the output from the model matches an observed set of data to an acceptable level. Therefore, calibration is an iterative process whereby model coefficients are adjusted until an adequate fit of observed versus predicted data is obtained. Unfortunately, there are no hard and fast guidelines for determining when an adequate fit is obtained. If a mode is not well fitted, then the user must consider if the inability of the model to produce useful results is due to the use of the model in an inappropriate manner, or the model formulations are insufficient to describe known prototype behavior, or the input data are insufficient to describe the system dynamics. Another important point during calibration is that a model may give inadequate results for a given spatial and/or temporal scale, but at another scale may reasonably represent the dynamics of the prototype. For example, the model may fail to predict a short-term phytoplankton bloom using monthly inflowing phytoplankton and nutrient concentrations, but may adequately represent phytoplankton production over the summer stratification period. The model may thus be useful in determining a water-body's long-term response to nutrient loading reductions but be inadequate in addressing short-term responses to a nutrient reduction strategy. In 
summary, it is not always necessary for model output to match all of the observed data for the model to provide meaningful results. The usual sequence for calibration is to first calibrate the water budget (or water surface elevation), then to calibrate temperature (in reservoirs), and finally, to calibrate water quality (Cole \& Wells, 2003).

As mentioned above calibration (or even worse, automatic calibration) may be useless and/or dangerous in some cases, and so is the belief that the outputs of the model should be adjusted as closely as possible to the measurements (Guinot and Gourbesville, 2003).

\subsubsection{Verification \& Validation vs. Confirmation}

Model verification and validation (V\&V) are essential parts of the model development process if models are to be accepted and used to support decision-making. Verification is done to ensure that the model is programmed correctly, the algorithms have been implemented properly, and the model does not contain errors, oversights, or bugs. Therefore, verification does not ensure that the model solves an important problem, meets a specified set of model requirements, or correctly reflects the workings of a real world process.

Validation ensures that the model meets its intended requirements in terms of the methods employed and the results obtained. The ultimate goal of model validation is to make the model useful in the sense that the model addresses the problem, provides accurate information about the system being modelled, and to makes the model actually to be used. In theory, the predictions of the model should be compared at all appropriate levels with different data obtained from real systems. However, this is rarely done in practice. In some cases, all or part of the calibration data is used again in the validation. Such a partial validation, using data from the same site and/or under similar conditions, is called model confirmation (Røed, 2005).

Mainly in the step of model development, V\&V work together by removing barriers and objections to the use of the model. In the case of models that contain elements of human decision making, validation becomes a matter of establishing credibility in the model. However, in the step of model application, confirmation is a substitute for V\&V. It is crucial that independent data sets from many different regimes are used to establish the model's credibility through rigorous statistical tests. There are many statistical tools for model confirmation, but the primary tool for most process modeling applications is graphical residual analysis. Numerical methods for model confirmation, such as the statistic, are also useful.

Obviously, if the observational data are very limited, then all of it is used in the calibration stage and even a model confirmation is not possible until further and preferably wider range of data sets become available. A variety of model fit statistics is available for evaluating model performance (Reckhow et al., 1990). Actually, calibration uses Goodness-of-Fit Statistics that include the following: 
1) r-correlation coefficient of the model predictions and observations

$$
r=\frac{\sum_{i=1}^{n}\left(O_{i}-\bar{O}\right)\left(P_{i}-\bar{P}\right)}{\sqrt{\sum_{i=1}^{n}\left(O_{i}-\bar{O}\right)^{2} \sum_{i=1}^{n}\left(P_{i}-\bar{P}\right)^{2}}}
$$

2) RMSE- the root mean squared error

$$
R M S E=\sqrt{\frac{\sum_{i=1}^{n}\left(P_{i}-O_{i}\right)^{2}}{n}}
$$

3) RI- the reliability index

$$
R I=\exp \sqrt{\frac{1}{n} \sum_{i=1}^{n}\left(\log \frac{O_{i}}{P_{i}}\right)^{2}}
$$

4) AE- the average error

$$
A E=\frac{\sum_{i=1}^{n}\left(P_{i}-O_{i}\right)}{n}=\bar{P}-\bar{O}
$$

5) AAE- the average absolute error

$$
A A E=\frac{\sum_{i=1}^{n}\left|P_{i}-O_{i}\right|}{n}
$$

6) MEF- the modeling efficiency

$$
M E F=\frac{\left(\sum_{i=1}^{n}\left(O_{i}-\bar{O}\right)^{2}-\sum_{i=1}^{n}\left(P_{i}-O_{i}\right)^{2}\right)}{\sum_{i=1}^{n}\left(O_{i}-\bar{O}\right)^{2}}
$$

where $n=$ number of observance; $O_{i}=i$ th of $n$ observances; $P_{i}=i$ th of $n$ predictions; $\bar{O}$ and $\bar{P}=$ observation and prediction averages, respectively. If all observations and predictions were log-transformed before calculating the fit statistics so that differences 
between predicted and observed values would not be highly skewed and dominated by a small portion of high values.

The correlation coefficient " $r$ " measures the tendency of the predicted and observed values to vary together linearly. It can range from -1 to 1 , with negative values indicating that the observed and predicted values tend to vary inversely. It should be recognized that even if the correlation is close to 1 , the predicted and observed values may not match each other, but they only tend to vary similarly.

The root mean squared error, average error, and average absolute error are all measures of the size of the discrepancies between predicted and observed values. Values near zero indicate a close match. The average error is a measure of aggregate model bias, though values near zero can be misleading because negative and positive discrepancies can cancel each other. The average absolute error and the root mean squared error both accommodate the shortcoming of the average error by considering the magnitude rather than the direction of each discrepancy. Together these three statistics provide an indication of model prediction accuracy.

The reliability index (Leggett and Williams 1981) quantifies the average factor by which model predictions differ from observations. An RI of 2.0 indicates that a model predicts the observations within a multiplicative factor of two, on average. Ideally, the RI should be close to one. When the root mean squared error has been calculated for log-transformed values of the predictions and observations, then the RI is the exponentiated RMSE.

The modeling efficiency measures how well a model predicts relative to the average of the observations. It is related to the RMSE according to $\mathrm{MEF}=1-\mathrm{RMSE}^{2} / \mathrm{s}^{2}$ where $\mathrm{s}^{2}$ is the variance of the observations. A value near one indicates a close match between observations and model predictions. A value of zero indicates that the model predicts individual observations no better than the average of the observations. Values less than zero indicate that the observation average would be a better predictor than the model results.

\subsubsection{Sensitivity Analysis}

The aim of a sensitivity analysis is performed to understand where more research or measurements are needed (priority setting). In essence, there are two distinct components of the sensitivity analysis; the first deals with the sensitivity to the input data and conditions, and the second involves the sensitivity to the chosen parameter values and to the parameterizations themselves (Røed, 2005). Therefore the sensitivity analysis is to determine whether the chosen model accurately reproduces conditions in the 'real world.' It comes from a determination of the effect of small changes in model parameters on the results, by either numerical simulation or mathematical techniques. Therefore it may be a form of uncertainty analysis, but it is not strictly such. The 
methods used in water quality modeling very often are described as follows.

\section{1) Parameter Perturbation}

We can assume a simple mass-balance equation for a well-mixed reservoir as follow:

$$
V \frac{d c}{d t}=Q_{i n} c_{i n}-k c V-Q_{o u t} c
$$

A steady state condition is $d c / d t=0$. So, the solution of Equation (4.28):

$$
c=\frac{Q_{i n}}{Q+k V} c_{i n}
$$

According to this formula $c$ is a function of each of the model parameters and forcing functions; that is, $c=f\left(Q, k, V, c_{i n}\right)$. Thus, one way of visualizing the dependence of the solution on one of the parameters (for example $k$ ) is by developing a plot of $c$ vs. $k$.

As the name implies, parameter perturbation consists of varying each of the model parameters (for example raised and lowered a fixed \%) while holding all other terms constant. Thus, the quantification of sensitivity of Equation (4.53) is calculated by:

$$
\Delta c=\frac{c(k+\Delta k)-c(k-\Delta k)}{2}
$$

\section{2) First-order Sensitivity Analysis}

This approach uses the derivative of the function with respect to the parameter as an estimate of the sensitivity. One way to derive this is to employ first-order Taylor-series expansions of Equation (4.30) around the values of the parameter. For example, forward and backward expansions can be written as

$$
\begin{aligned}
& c(k+\Delta k)=c(k)+\frac{\partial c(k)}{\partial k} \Delta k \\
& c(k-\Delta k)=c(k)-\frac{\partial c(k)}{\partial k} \Delta k
\end{aligned}
$$

If Equation (4.31) and (4.32) are input to Equation (4.30):

$$
\Delta c=\frac{c(k+\Delta k)-c(k-\Delta k)}{2}=\frac{\partial c(k)}{\partial k} \Delta k
$$

A refinement on both the perturbation and first-order sensitivity analyses is to express 
the results in terms of a condition number $C N$ (Chapra and Canale 1988). For the firstorder sensitivity analysis, such a number can be derived by dividing both sides of Equation (4.33) by $c$ :

$$
\frac{\Delta c}{c}=C N_{k} \frac{\Delta k}{k}
$$

where, $C N_{k}$ is the condition number for the parameter $k$,

$$
C N_{k}=\frac{k \partial c}{c \partial k}
$$

Equation (4.35) indicates that the condition number provides a transfer function to propagate the relative error of the parameter into the relative error of the prediction. Note that although the derivation is for the first-order sensitivity analysis, it can be used for the perturbation analysis. However, in this case a discrete form is used for the derivative:

$$
C N_{k}=\frac{k \Delta c}{c \Delta k}
$$

\subsubsection{Uncertainty/Variability Analysis}

Uncertainty refers to a random prediction error resulting from limitations in the data and in models used to formulate the lake nutrient balance, trophic response, and/or the performance of measures to achieve the allocated loads (Walker and Havens, 2003).

Models include the following types of error (Schnoor, 1996):

- model error (differential equation and unforeseen reactions).

- errors in the state variables (dependent variables and initial conditions).

- errors in the input data used to derive the model.

- parameter error (rate constants, coefficients, and independent variables).

Model error is the most difficult type to consider in uncertainty analysis, but one approach is to use several different models for the same application. Then one can obtain an estimate of model error by virtue of the differences in predictions. If a model performs better under certain circumstances, one can discover the cause of model error.

Other errors in state variables, input data, and parameters are best addressed through the following method: sensitivity analysis, first-order analysis, or Monte Carlo simulations.

Despic and Simonovic (1997) identified two basic forms uncertainty in environmental 
modeling: uncertainty caused by inherent stochastic variability and uncertainty due to a fundamental lack of knowledge (including model structure and model parameters).

Stochastic variability includes two concepts: temporal and/or spatial variations in a whole water body, and variability due to inexact prediction. The former (type I) refers to temporal and/or spatial variations in water quality generated by turbulence and/or wind stress fluctuations, and it is more difficult than uncertainty to predict and handle. Uncertainty can be reduced by more data, very scrupulous model construction and efforts in reducing parameter error, whereas the stochastic temporal and spatial variability cannot be introduced into what are deterministic models. Thus, the approaches to the variability adopt compartmental time series deterministic modeling and/or dynamic stochastic modeling.

The latter (type II) is mainly oriented to uncertainty as follows. Most water quality models predict a single value at a point in time and space. However, the relationship between pollutant loading and receiving water effects can never be perfectly known by a model. Regardless of the accuracy and complexity of the modeled physical, chemical, and biological processes, there will be residual uncertainty due to natural variation, misspecification of boundary conditions and measurement error. In recent researches, there were attempts to develop a statistical assessment of violations of water quality standards, in other words, to predict the frequency of water quality standard violations (Duan et al., 2006). This was based on probabilistic approaches based on Bayesian theory.

Many methods of uncertainty analysis are reported and used in modelling areas. In particular, three methods, which have been most frequently used in the water quality modelling, are presented in this section: Monte Carlo simulation, Probabilistic method, and Fuzzy sets.

\section{1) Monte Carlo Simulation}

It is conceptually very simple. One uses a random number generator to select parameter values from a known or suspected distribution (probability density function). After the proper number of parameters has been randomly selected (synthetic sampling), the model is run and the results of the simulation are saved. Then the process is repeated until a statistically significant number of simulations (large number of realizations) have been recorded. From the distribution of results, a mean predicted value and a standard deviation could be calculated that reflects the combined uncertainty of all parameter errors.

The advantages of Monte Carlo simulation are that it does not require linearization of the model equations and it is, in theory, nonparametric. Error terms in parameters and independent variables can be represented as any probability density functions and sampled without the requirement of standard deviation and mean (Gaussian) statistics. However, Monte Carlo analysis is computationally expensive for large models, and it does not preserve the covariance structure among the parameters (unless one performs 
the synthetic sampling in a special manner to obtain a realistic set of outcomes).

Parameter distributions may be determined by multiple samples collected in the field, determined in microcosm experiments in the laboratory, or from expert judgment. Typical distributions of parameters are normal distributions, lognormal, "hat" functions (i.e., constant probabilities over the range of parameter values), and trapezoid functions (i.e., constant probabilities in a predominant range and decreasing linearly at both ends of the function). Normal distributions are thought to arise from "additive" processes in the environment.

$$
f(\mathrm{x})=\frac{1}{\sigma \sqrt{2 \pi}} \exp \left[-\frac{1}{2}\left(\frac{\mathrm{x}-\mu}{\sigma}\right)^{2}\right]
$$

where $\mu$ is the mean value of the distribution and $r$ is the standard deviation. Equation (4.37) gives the probability density function (pdf) for a normal distribution. It is a bellshaped Gaussian distribution. The area under the curve at $x= \pm 1 \sigma$ contains $68.3 \%$ of the values; the area under the curve at $x= \pm 2 \sigma$ contains $95.5 \%$ of values.

Perhaps the most common distribution of parameters in the environment is the lognormal distribution. If you do not know anything about how a parameter varies, you should assume a lognormal distribution. It accounts for environmental measurements with skewness in their distribution, which is often the case. Lognormal distributions are thought to arise from multiplicative processes. They can be made into a Gaussian normal distribution simply by performing a log transformation on the data. Equation (4.38) is the pdf for a lognormal distribution:

$$
f(y)=\frac{1}{y \sqrt{2 \pi} \sigma_{x}} \exp \left[-\frac{1}{2}\left(\frac{\ln y-\mu_{x}}{\sigma_{x}}\right)^{2}\right]
$$

where $\mu_{x}$ is the geometric mean of the parameter values, $\sigma_{x}$ is the geometric deviation, and $y$ are the parameter values themselves, which must be positive. The $y$ values are transformed by taking their natural logarithm.

$$
x=\ln y
$$

where $\mathrm{y} \geq 0$ and $-\infty \leq \mathrm{x} \leq+\infty$. The area under a probability density function is normalized to 1.0 , so the use of pdf's allows a ready comparison to be made in the shape of various parameters.

Monte Carlo analysis offers some advantages over first-order analysis. Due to the low unit cost of computing, it has become the favored method among environmental scientists and engineers for uncertainty analysis. 


\section{2) Probabilistic Method}

The use of a limited sample for statistical inference introduces uncertainty into the assessment of compliance (Borsuk et al., 2001). Rules for compliance are generally derived from classical one-sided hypothesis tests in which the p-value of exceedances of a limit in percentile is used as a weight-of-evidence measure against the tested hypothesis.

\section{a) p-value}

A compliance rule generally requires that no more than a stated number of exceedances of the percentile limit should occur in a given number of samples over a compliance assessment period. Therefore, the compliances of exceeding a certain limit (a numerical standard) $c$ can be developed by following procedures (Borsuk et al., 2001).

The general mass balance equation can be expressed as:

$$
V \frac{d c}{d t}=W(t)-Q c \pm k V c
$$

In the Equation (4.40), concentration $c$ is the dependent or response variable, and $W(t)$ is the independent, or input variable. The quantity $V, Q, k$, and $V$ are the parameters or coefficients of the model. The Equation (4.40), which has solutions by either analytically or numerically, can be written in the general form:

$$
c=g(X, \beta)+\varepsilon
$$

where $g$ is a mathematical function (or functions) relating the pollutant concentration $c$ to a set of all input variables $X$ and a set of all model parameters $\beta$. The term $\varepsilon$ represents an error that accommodates discrepancies between the predicted values of $c$ represented by $g(X, \beta)$ and the observed values of $c$. The equation is entirely general and represents a series of detailed process-based functions (physically based model) or the expression of statistical relationships derived from historical data.

Characterization and incorporation of the residual error term is straightforward for a model that has been optimized to available data using maximum likelihood or leastsquares regression. Adherence to the assumptions required for statistical inference with least-squares regression implies that the residual errors follow a normal distribution (after a suitable transformation, if necessary) with a mean of 0 and a variance, $\sigma^{2}$, the value of which is directly estimated from the data and is assumed to be a constant with respect to the value of $c$. Therefore, the response variable $c$ about a given set of input variables $X$ and model parameters $\beta$, is a normal distribution with a mean $g(X, \beta)$ and a variance $\sigma^{2}$ :

$$
c \sim N\left(g(X, \beta), \sigma^{2}\right)
$$


The probability of the response variable exceeding a numerical criterion $c^{*}$, can be calculated as:

$$
p=\operatorname{Pr}\left(c>c^{*} \mid \beta, \sigma, X\right)=1-F\left(\frac{c^{*}-g(X, \beta)}{\sigma}\right)
$$

where

$p=$ probability of exceedance

$c^{*}=$ numerical criterion

$\beta=$ parameters

$X=$ input variables

$\sigma=$ standard deviation of parameters

$F()=$ value of the cumulative standard normal distribution

$g=$ mathematical function relating the nutrient concentration c

\section{b) Non-probabilistic Numerical Method}

This approach of uncertainty analysis is based on nonlinear and sophisticated datadriven techniques such as artificial intelligence, neural networks and fuzzy logics.

The use of these methods is particularly when the physical world is not fully understood, when the model has many uncertainties (model coefficients and/or input parameters), and when there is extensive data for training the network. This is because when the extensive significant data exist, these methods can provide a methodology for dealing with uncertainty through learning based on patterns in the available data (Ross, 1995). Therefore, this modelling approach has recently emerged as an alternative approach to simple empirical approaches and complex deterministic knowledge-based models.

\section{3) Fuzzy-set}

In the fuzzy case, for each value of measurement error $\Delta x_{i}$, we describe the degree $\mu_{i}\left(\Delta x_{i}\right)$ to which this value is possible. For each degree of certainty $\alpha$, we can determine the set of values of $\Delta x_{i}$ that are possible with at least this degree of certainty - the $\alpha$-cut $\{x \mid \mu(x) \geq \alpha\}$ of the original fuzzy set. Vice versa, if we know $\alpha$-cuts for every $\alpha$, then, for each object $x$, we can determine the degree of possibility that $x$ belongs to the original fuzzy set.

A fuzzy set can be thus viewed as a nested family of its $\alpha$-cuts. If instead of a (crisp) interval $x_{i}$ of possible values of the measured quantity, we have a fuzzy set $\mu_{i}(x)$ of possible values, then we can view this information as a family of nested intervals $\mathrm{x}_{i}(\alpha)$ $\alpha$-cuts of the given fuzzy sets.

The objective is then to compute the fuzzy number corresponding to this the desired value $y=f\left(x_{1}, \ldots \ldots \ldots, x_{n}\right)$. In this case, for each level $\alpha$, to compute the $\alpha$-cut of this fuzzy number, we can apply the interval algorithm to the $\alpha$-cuts $x_{i}(\alpha)$ of the corresponding fuzzy sets. The resulting nested intervals form the desired fuzzy set for $y$ (Kreinovich et al.). 


\subsubsection{Quality Assurance}

In the modelling project, there is a cycle of three steps: modeling needs and requirements analysis, modelling design, and model application. Strictly speaking, quality assurance, or QA for short, of modelling should be carried out through the whole cycle.

According to the definition of QA, it refers to planned and systematic production processes that provide confidence in a product's suitability for its intended purpose (Fox, 2008). In the water management field, model developers and constructers, and data analyzers etc are responsible for QA of modelling. In this study, the objective of QA is a series of instantiated models to ensure that the modelling results coincide with the purpose of modelling, but is not concerned with the modelling needs and requirements analysis, or the model design. Therefore, only a model operator who has experience and knowledge of the physics and understands how reality is described in the model should operate a model.

Unfortunately, there are no guidelines and fixed rules for QA because of uncertainties in the model codes, governing equations, solution methods, and raw data etc. However, there are principles to characterise QA: 'modeling framework' (the model and its supporting data availability and instantiating procedure are defensible and appropriate for its planned final use), 'right first time' (mistakes should be minimized as far as possible or eliminated) and 'fit for purpose' (the product should be suitable for the intended purpose) (US EPA, 2002).

Therefore a total quality control for QA in the model application step are summarised such as Figure 4.7. This is the procedure of instantiating models mentioned above.

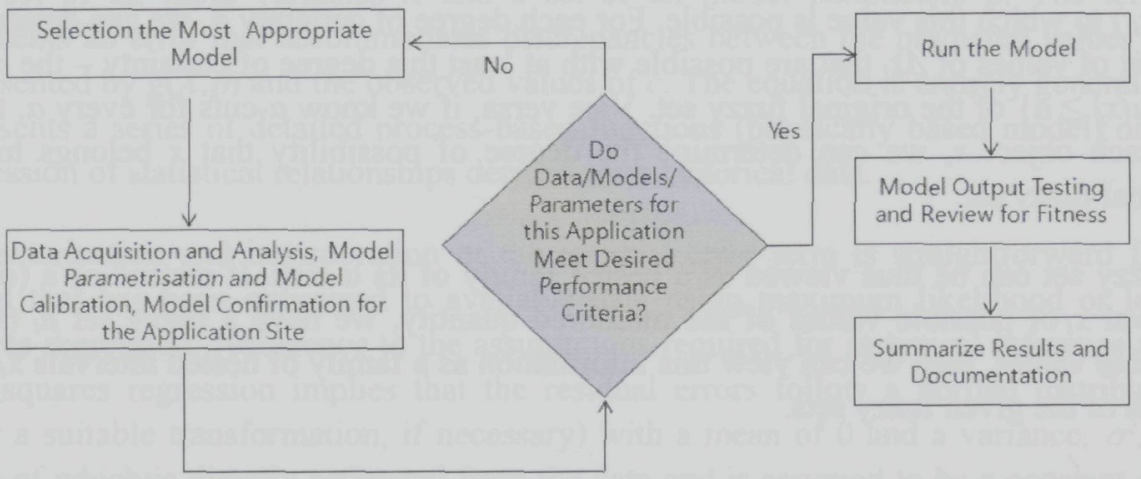

Figure 4.7. Procedure of modelling quality assurance. 


\section{Chapter 5}

\section{DATA-DRIVEN MODELLING}

\section{Summary}

There are various different types of model that can be induced as large amounts of collected data. These data driven models (DDM) are used in hydroinformatics to deduce information from data sets using machine learning (ML) methods. In this chapter three machine learning methods, including the partition rule (or tree or numerical regression), the instance based rule of $k$ number(s), and the clustering rule are described. An artificial neural networks (ANNs) is an agent classifier (a kind of regression); $k$ nearest neighbor $(k \mathrm{NN})$ is an instance-based rule; and model trees (MTs) uses a rule of partition. MT is recognized as one of the most explicit DDM methods, because it consists of tree-structured regression sub-models that associate leaves with multiple linear regression functions. The clustering method attempts to find natural groups of components (or data) based on some similarity feature. It identifies instances that bear a stronger resemblance to each other rather than to remaining instances. The partial least square regression (PLSR) method finds latent vectors for each cluster that explain both the response and predictor variations. Therefore, the PLSR method is well suited to regression when the data is highly correlated and where there is only a limited number of observations. This is because it balances the two objectives of explaining variations in the response and the predictor.

\subsection{Introduction}

\subsubsection{Definition}

In the interdisciplinary area of exploring a large quantity of data in order to discover meaningful information from it, in the form of patterns and rules, different terminologies are used: data mining (DM); artificial intelligence (AI), including artificial neural networks (ANNs) and genetic algorithms (GAs); computational intelligence (CI); fuzzy logic (FL); intelligent data analysis (IDA); data-driven modelling (DDM); soft computing etc. In fact, DM and DDM together cover all of these terminologies.

DDM as defined by Solomatine (2002) is the approach to modelling that focuses on using machine learning (ML) methods to draw information from data sets in order to build models (often of a natural system) that complement or replace the "knowledgedriven" models that are based on descriptions of the basic processes of a physical system. A DDM works with data from the boundaries of the domain of interest, and finds a form of relationship(s) that best connects specific data sets (Price, 2002). 
Consequently DDM is used in hydroinformatics to induce information from large quantities of data and then to predict trends.

ML should be differentiated from DDM in terminology, because this is a subfield of DDM, i.e. a part of DDM known as a training algorithm. There are many algorithms in ML such as FL, and descriptive and inferential statistics for classification, association, clustering and regression. Therefore DDMs have been developed by adopting an ML algorithm for a particular application area.

DDMs are not restricted to pattern recognition; they provide an opinion about data, and as with any opinion, its viewpoint can be altered as new data is introduced. Part of the 'closed loop' approach to decision support is that all of the steps within the loop can be increasingly improved as more information becomes available. A DDM can be retrained with more and better data as it becomes available, further increasing the performance of such a model.

DDMs and data warehouses complement each other. Well-designed data warehouses handle the data selection, cleaning, enrichment, and transformation steps that are also typically associated with DDMs. Similarly, the process of data warehousing improves the appreciation of the data because with the development and application of DDMs, it becomes apparent which data elements are considered more valuable than others in terms of decision support. Corresponding improvements to data cleaning and transformation steps are crucial to good data warehousing practice. DDMs, through their analysis of historical data, do not guarantee the nature of future data. Instead, DDMs are guidance tools, used to provide insight into trends inherent in the historical information. Although one can peruse the structure of a DDM to understand more about the patterns and rules that are inherent to the historical data, the real power of a DDM comes from using it as a predictive vehicle with current data.

One can use the DDM as a lens through which to view current data, with the ability to apply the patterns and rules stored in the model to predict trends in such data. The revealed information can then be used for decision making. Furthermore, the feedback from such decision making can be compared with the predicted results of the DDM to improve further the patterns and rules stored in the model itself, which can then be used to predict more accurately trends in new data, and so on.

\subsubsection{Data-Driven Modelling and Science}

Data mining or DDM has become fashionable, not just in computer science, but particularly in business IT and many research fields. The emergence is originally due to the growth in data warehouses and the realisation that this mass of operational data has the potential to be exploited as an extension of business intelligence (Sumathi and Sivanandam, 2006). If so, how scientific is DDM and is it worth using it in research area? The summary that Read (1999) gave to about this question in his article: "Data Mining and Science?" is discussed here. 
Rules generated by data mining are not physical laws, but empirical. In most modelling research areas, one compares observed data with modelling results that are the output from the simulation of a model based on temporal and spatial difference equation of physical laws. The success or otherwise of the comparison is a test of the hypothesis of how nature works as expressed by a mathematical formula. At this time, fitting a mathematical model to the data might determine physical parameters.

On the other hand, where there are no general theories, data mining techniques are valuable in identifying the underlying relationship(s), especially where there is a large quantity of data containing noisy patterns. This approach seeks to obtain a theoretical generalisation automatically from the data by means of induction, deriving empirical models and learning from example. The resultant theory, while it may not be fundamental, can yield a good description of the physical process and can have great practical utility.

A common approach is to use data instances or cases to generate an empirical algorithm that makes sense to the scientist and that can be put to practical use for recognition or prediction. Consequently, DDMs are making induced information from large data sets accessible in scientific disciplines, such as bio-informatics, medical science, astronomy etc.

\subsection{Machine Learning Methods}

DDM is about discovering meaningful information by analyzing large amounts of data already present in databases. In other words, the aim is to discover subtle relationships between data variables, and to construct predictive models based on them. For this, DDM borrows machine learning techniques from statistics and artificial intelligence in cooperation with computer science.

The tasks well-suited for DDM are defined as: association, clustering, regression and partition. Some of these tasks are best approached in a top-down manner called hypothesis testing. In hypothesis testing, a database recording past behavior is used to verify or disprove preconceived notions and ideas in the data. Other tasks, involving machine learning algorithms, are best approached in a bottom-up manner called knowledge discovery. In knowledge discovery, no prior assumptions are made; the data is allowed to speak for itself. Knowledge discovery comes into two categories for machine learning: statistics and artificial intelligence cooperating with computer science.

Machine learning techniques based on statistics, use rules of clustering and association to discover the relationships between attributes in unknown data, with or without access to known data with which to compare the outcome. There may or may not be a specific scenario. Clustering and association, for example, are primarily DDM techniques. This approach is best employed in situations in which true data discovery is needed to uncover rules and patterns in unknown data. This tends to be the "I don't know what I don't know" approach: we can discover significant attributes (variables) and patterns in a diverse set of data without using training data or a predefined scenario. These kinds of 
DDM are treated as a 'white box' operation, in which the user is concerned about both the process used by the data mining algorithm to create the model and the results generated by viewing the data through the model.

Machine learning techniques based on partition or regression (tree and numerical prediction) provides the technical basis of DDM. Regression is a machine learning technique used to predict group membership for data instances: whether to ANNs, FL and GA. This approach is best employed when a clear scenario can be employed against a large body of known historical data to construct a predictive DDM. This tends to be the "I know what I don't know" approach: we have a good idea of the specific scenarios to be modeled, and have solid data illustrating such scenarios, but we are not sure about the outcome itself or the relationships that lead to this outcome. These kinds of DDM are treated as 'black boxes', in which the user cares less about the model and more about the predictive results that can be obtained by viewing the data through the model.

DDM depends on both kinds of machine learning techniques to be truly effective, depending on what questions are asked and what data is analyzed. They can be employed separately or together, in varying amounts, depending on one's business requirements. Until now most DDMs use the second machine learning method known as partition.

\subsubsection{Regression and Partitioning}

\section{1) Artificial neural network}

An artificial neural network (ANN) is an information processing system that replicates the behaviour of a human brain by emulating in a rudimentary way the operations and connectivity of biological neurons. It consists of a number of neurons, i.e. simple linear on nonlinear computing elements, interconnected in complex ways and organized into layers. ANNs are used in three main ways:

- as models of biological nervous systems and intelligence

- as real-time adaptive signal processors or controllers implemented in hardware for applications such as robots

- as data analytic methods.

Here we use ANNs primarily for data analysis. An ANN, like many statistical methods, is capable of processing vast amounts of data and making predictions that are sometimes surprisingly accurate; this does not make them "intelligent" in the useful sense of the word. The ANN learns in much the same way as many statistical algorithms, but usually much more slowly. ANNs and statistics are not competing methodologies for data analysis. There is considerable overlap between the two fields. ANNs include several models, such as multi-layer perceptrons (MLPs), which are useful for statistical applications. Statistical methodology is directly applicable to neural networks in a variety of ways, including estimation criteria, optimization algorithms, confidence 
intervals, diagnostics, and graphical methods. Better communication between the fields of statistics and artificial neural networks would benefit both.

From a mathematical point of view, an $\mathrm{ANN}$ is a complex non-linear function with many parameters that are adjusted (calibrated, or trained) in such a way that the ANN output becomes similar to the measured output of a known data set.

The true power and advantage of neural networks lie in their ability to represent both linear and non-linear relationships and in their ability to learn these relationships directly from the data being modeled. Traditional linear models are simply inadequate when it comes to modelling data that contains non-linear characteristics. Two particular ANNs namely MLP and radial basis function (RBF) are summarized by Sarle (1994) as follows.

\section{a) Multi-layer Perceptron (MLP) ANN}

The multi-layer perceptron (MLP) is the most common neural network. This type is known as a supervised network because it requires a desired output in order to learn. The goal of this type of network is to create a model that correctly maps the input to the output using historical data so that the model can then be used to produce the output when the desired output is unknown

\section{(1) Network Diagrams}

Various models can be displayed as network diagrams such as the one shown in Figure 5.1 which illustrates the ANN and statistical terminology for a simple linear regression model. Neurons are represented by circles and boxes, while the connections between neurons are shown as arrows:

- Circles represent observed variables, with the name shown inside the circle.

- Boxes represent values computed as function of one or more arguments. The symbol inside the box indicates the type of function. Most boxes also have a corresponding parameter called a bias.

- Arrows indicate that the source of the arrow is an argument of the function computed at the destination of the arrow. Each arrow usually has a corresponding weight or parameter to be estimated.

- Two long parallel lines indicate that the values at each end are to be fitted by least squares, maximum likelihood, or some other estimation criterion.

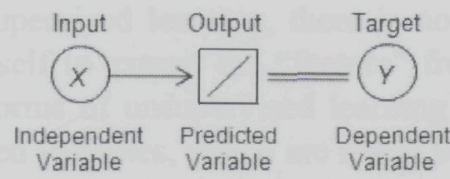

Figure 5.1. Simple linear regression.

\section{(2) Perceptrons}

A perceptron computes a small linear combination of the inputs called the net input. Then a possibly nonlinear activation function is applied to the net input to produce the output. An activation function maps any real input into a usually bounded range, often 0 
to 1 or -1 to 1 . Some common activation functions are:

- linear or identity: $\operatorname{act}(x)=x$

- hyperbolic tangent: $\operatorname{act}(x)=\tanh (x)$

- logistic: $\operatorname{act}(x):=\left(1+e^{-x}\right)^{-1}=(\tanh (x / 2)+1) / 2$

- threshold: $\operatorname{act}(x)=0$ if $x<0,1$ otherwise

- Gaussian: $\operatorname{act}(x)=e^{-x^{2} / 2}$

Symbols used in the network diagrams for various types of neurons and activation functions are shown in Figure 5.2. A perceptron can have one or more outputs. Each output has a separate bias and set of weights. Usually the same activation function is used for each output, although it is possible to use different activation functions.

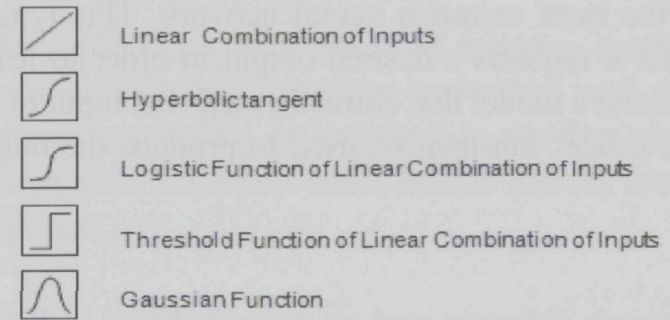

(3) Multilayer Perceptrons (MLP)
Figure 5.2. Commonly used activation function.

A functional link network introduces an extra hidden layer of neurons, but there is still only one layer of weights to be estimated. If the model includes estimated weights between the inputs and the hidden layer, and the hidden layer uses nonlinear activation functions such as logistic function, the model becomes genuinely nonlinear, i.e., nonlinear in the parameters. The resulting model is called a multilayer perceptron or MLP. An MLP can have multiple inputs and outputs, as shown Figure 5.3.

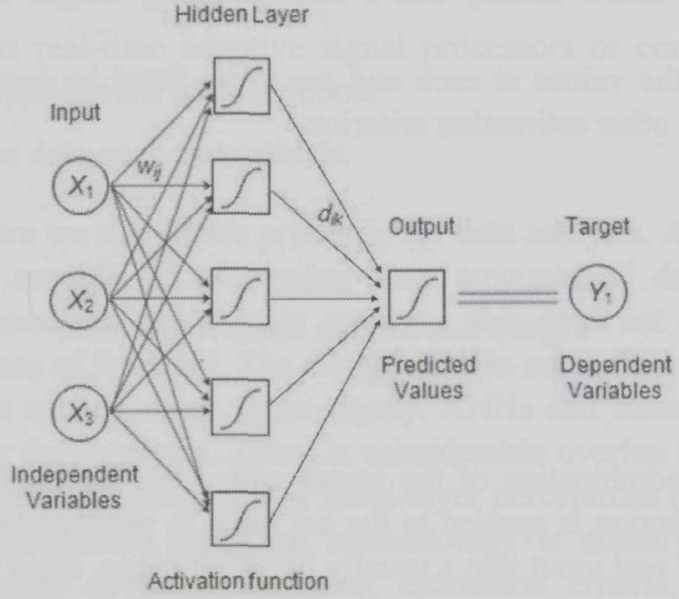

Figure 5.3. Multilayer Perceptron ANN. 
The notation and formulae for an MLP as shown in Figure 2 are as follows:

$\begin{array}{ll}n_{x} & =\text { number of independent variables (inputs) } \\ n_{h} & =\text { number of hidden neurons } \\ x_{i} & =\text { independent variable (input) } \\ a_{j} & =\text { bias for hidden layer } \\ w_{i j} & =\text { weight from input to hidden layer } \\ g_{i} & =\text { net input to hidden layer }=a_{j}+\sum_{i=1}^{n_{k}} w_{i j} x_{i} \\ h_{j} & =\text { hidden layer values }=a c t h\left(g_{j}\right) \\ c_{k} & =\text { bias foe output (intercept) } \\ d_{j k} & =\text { weight from hidden layer to output } \\ q_{k} & =\text { net input to output layer }=c_{k}+\sum_{j=1}^{n_{h}} d_{j k} h_{k} \\ p_{k} & =\text { predicted value (output values) }=a c t_{o}\left(q_{k}\right) \\ y_{k} & =\text { dependent variable (training values) } \\ r_{k} & =\text { residual (error) }=y_{k}-p_{k}\end{array}$

where $a c t_{h}$ and $a c t_{o}$ are the activation functions for the hidden and output layers, respectively. The bias is used to shift the input to the transfer function, which is a numeric value typically between -1 and 1 or -0.5 and 0.5 added to the sum of the weighted inputs. When a neural network is initialized, all the weights are assigned a random value between -1 and 1 or -0.5 and 0.5 , which means that the bias $w_{i j}$ used in a Feed Forward Neural Network is initially assigned a small random value between -1 and 1 or -0.5 and 0.5 .

\section{(4) Learning}

The ANN has supervised and unsupervised learning. In supervised learning, the goal is to predict one or more target variables from one or more input variables. Supervision consists of the use of target values in training. Supervised learning is usually some form of regression or discriminating analysis. MLP is the most common variety of supervised network because it requires a desired output in order to learn. The goal of this type of network is to create a model that correctly maps the input to the output using historical data so that the model can then be used to produce the output when the desired output is unknown.

In unsupervised learning, there is no target variable, and the network is supposed to train itself to extract the "feature" from the independent variable. In fact, the goal in most forms of unsupervised learning is to construct feature variables from which the observed variables, which are really both input and target variables, can be predicted. In most cases, the dependent variables are predicted by linear regression from the feature variables.

Two typical learning algorithms for artificial neural networks are backpropagation and counterpropagation. The backpropagation algorithm adjusts the weights in the sums at each layer, in order to reduce the discrepancies between the network outputs before 
adjustment, and the desired values. The process of updating the network weights takes information from the set of training values and from the current network output, and uses these to adjust all the network weights. Thus, in spite of the fact that the "network" is feed-forward, the training process adds unequivocal and global feedback. The feedback path looks like that shown in Figure 5.4.

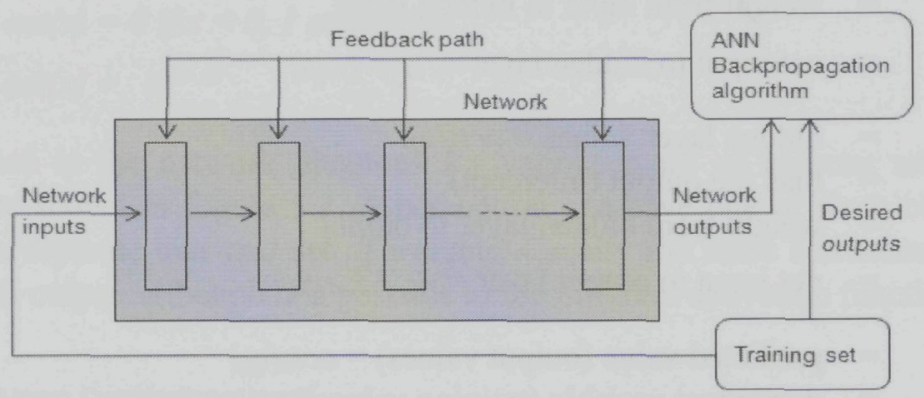

Figure 5.4. Schematic diagram of backpropagation.

The most common ANN is the error backpropagation network. Backpropagation is the way of using known input-output pairs of a target function to find the weights that make a certain mapping function approximate the target function as closely as possible. The mechanics of backpropagation neural networks and their gradient descent learning algorithm are now quite well known. Only a brief introduction to this type of neural networks is presented here.

The task faced by a backpropagation neural network is that of learning a supervised mapping: given a set of input vectors and associated target vectors, the objective is to learn a rule that captures the underlying functional relationship between the input vectors and the target vectors. Mathematically, each target vector $z$ is a function, $f$, of the input vector $x: z=f(x)$. The task of the backpropagation network is to learn the function $f$. Learning is achieved by finding regularities in the input patterns that correspond to the regularities in the output patterns. The network has a weight parameter vector, whose values are changed to modify a function $f$ computed by the network to be as close as possible to $f$. The weight parameters are determined by the learning rule known as gradient descent.

Counterpropagation networks are widely advertised as hybrid networks that learn much more rapidly than backpropagation networks. In counterpropagation networks, the variables are divided into two sets, say $x_{1}, \ldots, x_{n}$ and $y_{1}, \ldots \ldots, y_{n}$. The goal is to be able to predict both the $x$ variables from the $y$ variables and the $y$ variables from the $x$ variables. The counterpropagation network effectively performs a cluster analysis using both the $x$ and the $y$ variables. To predict $x$ given $y$ in a particular observation, compute the distance from the observation to each cluster mean using only the $y$ variables, find the nearest cluster, and predict $x$ as the mean of the $x$ variables in the nearest cluster. Therefore the counterpropagation algorithm can be inefficient and/or non-convergent in 
the case of lower relationships between $x$ and $y$ variables. The method for predicting $y$ given $x$ obviously reverses the roles of $x$ and $y$.

\section{b) Radial Basis Function (RBF) ANN}

A radial basis function (RBF) is another type of feed-forward ANN. Typically an RBF network has three layers: input, hidden and output. Unlike the backpropagation networks, there cannot be more than one hidden layer. The hidden layer uses a radial basis (Gaussian) function instead of other functions in Figure 5.5. A major advantage of RBF networks is that if the number of input variables is not too high, then learning is much faster than with other types of networks. However, the required number of hidden units in the layer increases geometrically with the number of input variables, so it becomes practically impossible to use this network for a large number of input variables.

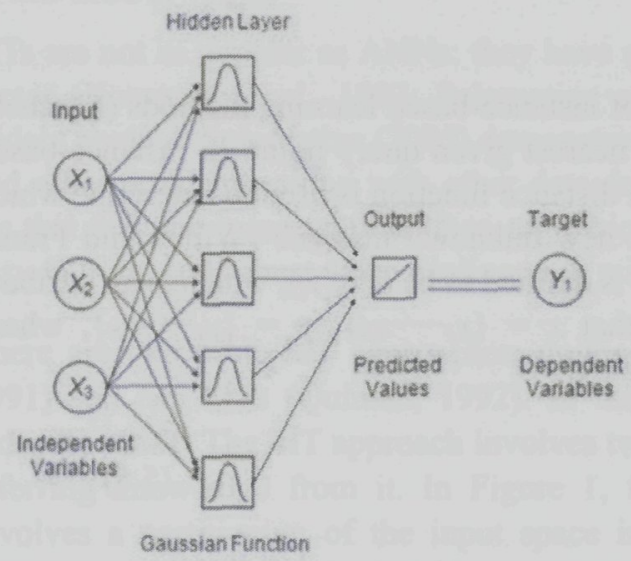

Figure 5.5. Radial Basis (Gaussian) Function ANN.

The net input to the hidden layer is the distance from the input vector to the weight vector, and is termed a "radial center" vector (Schalkoff, 1997). There is usually a bandwidth, $s_{j}$ (often termed sigma), associated with each hidden node. The activation function can be any of a variety of functions on non-negative real numbers with a maximum at zero, approaching zero at infinity (such as $e^{-x^{2} / 2}$ ). The outputs are computed as linear combinations of the hidden values with an identity activation function. For comparison, typical formulas for an MLP hidden neuron and an RBF neuron are as follows:

$$
\begin{array}{ll}
\text { MLP: } & g_{j}=a_{j}+\sum_{i=1}^{n_{x}} w_{i j} x_{i} \\
& h_{j}=\left(1+e^{g}\right)^{-1} \\
\text { RBF: } & g_{j}=\left[\sum_{i=1}^{n_{\mathrm{X}}} \frac{\left(w_{i j}-x_{i}\right)^{2}}{2 s_{j}}\right]^{1 / 2}
\end{array}
$$




$$
h_{j}=e^{-g_{j}^{2} / 2}
$$

Since an RBF network can be viewed as a nonlinear regression model, the weights can be estimated by any of the usual methods for nonlinear least squares or maximum likelihood, although this would yield a vastly over-parameterised model if every observation were used as an RBF centre. Usually, however, RBF networks are treated as hybrid networks. The inputs are clustered, and the RBF centres are set equal to the cluster means. The bandwidths are often set to the nearest-neighbor distance from the centre (Moody and Darken 1988), although this is not a good idea because nearestneighbor distances are excessively variable; it works better to determine the bandwidths from the cluster variances. Once the centres and bandwidths are determined, estimating the weights from the hidden layer to the outputs reduces to linear least squares.

\section{2) $k$-Nearest Neighbor ( $k N N)$}

$k$-Nearest Neighbor is the most basic type of instance-based learning methods (Mitchell, 1997) which locates $k$ spatial objects to a nearest given query point. In instance-based learning, training instances are stored and a distance function is used to determine which instance of the training set is closest to a new unknown instance (Witten and Frank, 2005). The distance between two instances is defined to be $d\left(x_{i}, y_{i}\right)$, which is an attribute of each instance with $N$ features, such that $x=\left\{x_{1} \cdots \cdots \cdot x_{N}\right\}, \mathrm{y}=\left\{y_{1} \cdots \cdots y_{N}\right\}$, where absolute distance measuring $d_{A}$ is expressed by:

$$
d_{A}\left(x_{i}, y_{i}\right)=\sum_{i=1}^{N}\left|x_{i}-y_{i}\right|
$$

and the Euclidean distance $d_{E}$ is expressed by:

$$
d_{E}\left(x_{i}, y_{i}\right)=\sum_{i=1}^{N} \sqrt{x_{i}^{2}-y_{i}^{2}}
$$

To find the closest instances, it is necessary to pass through the data set, one instance at a time, and compare it to the query instance. We can represent the data set as a matrix $D$ $=N \times P$, containing $P$ instances $s^{I}, \cdots s^{P}$, where each instance $s^{i}$ contains $N$ features $s^{i}=$ $\left\{s_{1}^{i}, \ldots \ldots, s_{N}^{i}\right\}$. A vector $o$ with length $P$ of output values $o=\left\{o^{i}, \cdots, o^{P}\right\}$ accompanies this matrix, listing the output values $o^{i}$ for each instance $s^{i}$. It should be noted that the vector $o$ can also be seen as a column matrix: if multiple output values are desired, the width of the matrix may be expanded.

The $k \mathrm{NN}$ algorithm consists of the following steps:

(1) store the output values of the $M$ nearest neighbors to query instance $q$ in vector $r=$ $\left\{r^{i}, \cdots, r^{M}\right\}$ by repeating the following loop $M$ times:

(a) go to the next instance $s^{i}$ in the data set, where $i$ is the current iteration within the domain $\{1, \cdots, P\}$ 
(b) if $q$ is not set or $q<d\left(q, s^{i}\right): q \leftarrow d\left(q, s^{i}\right), t \leftarrow o^{i}$

(c) loop until reaching the end of the data set (i.e. $i=P$ )

(d) store $q$ into vector $c$ and $t$ into vector $r$

(2) calculate the arithmetic mean output $\bar{r}$ across $\mathrm{r}$ as follows:

$$
\bar{r}=\frac{1}{M} \sum_{i=1}^{M} r_{i}
$$

(3) return $\bar{r}$ as the output value for the query instance $q$.

\section{3) M5 Model Trees}

MTs are not as popular as ANNs: they have only recently been introduced in the water sector (Kompare et al., 1997; Solomatine and Dulal, 2003), and have not yet been widely applied. Solomatine (2005) demonstrated the application of MTs to hydrological and other problems, along with other data-driven models. The advantages of M5 MTs are that they are more accurate than regression trees, more understandable than ANNs, easy to train, and robust when dealing with missing data (Witten and Frank, 2005).

There are two basic MT approaches: multiple adaptive regression splines (Friedman, 1991) and M5 MTs (Quinlan, 1992). In this study, the M5 algorithm was used for inducing a MT. The MT approach involves two major procedures: building the tree and inferring knowledge from it. In Figure 1, for example, the tree-building procedure involves a partitioning of the input space into mutually exclusive regions using the linear regression model. In the inference procedure, a new instance is fed into one of the models at the leaves of the tree, according to a splitting condition adopted in the treebuilding procedure, and then the predicted output is obtained from the linear model in the leaf. There is a version of the M5 algorithms known: M5' algorithm proposed by Wang and Witten (1997). This algorithm has a similar structure to the M5 algorithm, but it is able to deal effectively with missing values and enumerated attributes. These algorithms have the following three main steps.

\section{a) Building the Tree}

The basic tree is formed using the splitting criterion, which treats the standard deviation of the class values that reach a node as a measure of the error at that node, and calculates the expected reduction in error as a result of testing each attribute at that node. The attribute that maximizes the expected error reduction is then selected. The standard deviation reduction (SDR) for M5 is calculated using the formula

$$
S D R=s d(T)-\sum_{i} \frac{\left|T_{i}\right|}{|T|} \times s d\left(T_{i}\right)
$$


where $s d$ is the standard deviation of the set of examples $T$ that reach the node, and $T_{i}$ is the set that results from splitting the node according to the chosen attribute.

The splitting process ceases when the class values of all the instances that reach a node vary by less than $5 \%$ of the standard deviation of the original instance set, or when only a few instances remain.

\section{b) Pruning the Tree}

An over-fitting problem can occur during an MT construction based on training data. Predictably, the accuracy of the tree for the training examples increases monotonically as the tree grows. However, this increases overfitting, so that the accuracy measured over the independent test examples first increases, then decreases. A method for reducing this problem is termed pruning.

For use in the smoothing process a linear model is also needed for each interior node of the tree, not just at the leaves. Prior to pruning, a model is calculated for each node of the un-pruned tree. The model takes the form

$$
y=x_{0}+x_{1} a_{1}+x_{2} a_{2}+\ldots+x_{\mathrm{k}} a_{\mathrm{k}},
$$

where $a_{1}, a_{2}, \ldots, a_{\mathrm{k}}$ are attribute values. The weights $x_{1}, x_{2}, \ldots, x_{\mathrm{k}}$ are calculated using a standard regression. However, only the attributes tested in the sub-tree below this node are used in the regression, because the other attributes which affect the predicted value have been taken into account in the tests that lead to the node.

The pruning procedure uses an estimate of the expected error that will be experienced at each node for the test data. First, the absolute difference between the predicted value and the actual output value is averaged for each of the training examples that reach the node. Because the trees have been built expressly for this data set, this average will underestimate the expected error for new cases. To compensate for this, the output value is multiplied by the factor $(n+v) /(n-v)$, where $\mathrm{n}$ is the number of training examples that reach the node and $v$ is the number of parameters in the model that represent the output value at that node. Therefore, this multiplication is done to avoid underestimating the error for new data, rather than the data against which it was trained. If the estimated error is lower at the parent, the leaf node can be dropped (Witten and Frank, 2005).

\section{c) Smoothing}

A final stage is to use a smoothing process to compensate for sharp discontinuities that inevitably occur between adjacent linear models at the leaves of the pruned tree, particularly for some models constructed from a small number of training instances.

The smoothing procedure described by Quinlan (1992) uses the leaf model to compute the predicted value, and that value is then filtered along the path back to the root, smoothing it at each node by combining it with the value predicted by the linear model for that node. This involves the calculation 


$$
p^{\prime}=\frac{n p+k q}{n+k}
$$

where $p^{\prime}$ is the prediction passed up to the next higher node, $p$ is the prediction passed to this node from below, $q$ is the value predicted by the model at this node, $n$ is the number of training instances that reach the node below, and $k$ is a constant. In general, smoothing increases the accuracy of the predictions.

In the application of M5, it is not clear how to deal with enumerated attributes and missing values. These factors are critical in real-world data sets that are encountered in practice, and to take account of them the SDR is further modified to

$$
S D R=\frac{m}{|T|} \times \beta(i) \times\left[s d(T)-\sum_{j \in\{L, R\}} \frac{\left|T_{j}\right|}{|T|} \times s d\left(T_{j}\right)\right]
$$

where $\mathrm{m}$ is the number of examples without missing values for that attribute, $T$ is the set of examples that reach this node, $\beta(i)$ is the correction factor calculated for the original attribute to which this synthetic attribute corresponds, and $T_{L}$ and $T_{R}$ are sets that result from splitting on this attribute (all attributes are now binary).

\subsubsection{Clustering}

\section{1) $k$-Means Clustering}

A non-hierarchical approach to forming good clusters is to specify a desired number of clusters, say, $k$, then assign each case (object) to one of $k$ clusters so as to minimize a measure of dispersion within the clusters. $k$-means clustering (MacQueen, 1967) is an algorithm to partition $n$ objects based on attributes into $k$ group $k<n$. A method commonly used to automatically partition a data set into $k$ groups proceeds by selecting $k$ initial cluster centers and then iteratively refining them as follows (Wagstaff $e t$ al., 2001):

- each instance $d_{i}$ is assigned to its closest cluster center,

- each cluster center $C_{j}$ is updated to be the mean of its constituent instances.

It is similar to the expectation-maximization algorithm for mixtures of Gaussians in that they both attempt to find the centers of natural clusters in the data. It assumes that the object attributes form a vector space. The objective it tries to achieve is to minimize total intra-cluster variance, or, the squared error function

$$
V=\sum_{i=1}^{k} \sum_{x_{j} \in S_{i}}\left(x_{j}-\mu_{i}\right)^{2}
$$


where there are $k$ clusters $S_{i}, i=1,2, \ldots, k$, and $\mu_{i}$ is the centroid or mean point of all the points $x_{j} \in S_{i}$.

$k$-Means Clustering is analogous to linear regression: the residuals are the distance of each point to the regression line. In clustering, the residuals are the distance between each point and its cluster center. The $k$-means algorithm starts by randomly assigning instances to the classes, computes the centers according to

$$
\mu_{i}=\frac{1}{N_{j}} \sum_{j=1}^{n}\left|x_{j}\right|
$$

then reassigns the instances to the nearest cluster's center, recalculates the centers, reassigns the instances, etc. until $V$ stops decreasing (or the centers stop moving). Figure 5.6 shows a two-dimensional example of clustering.

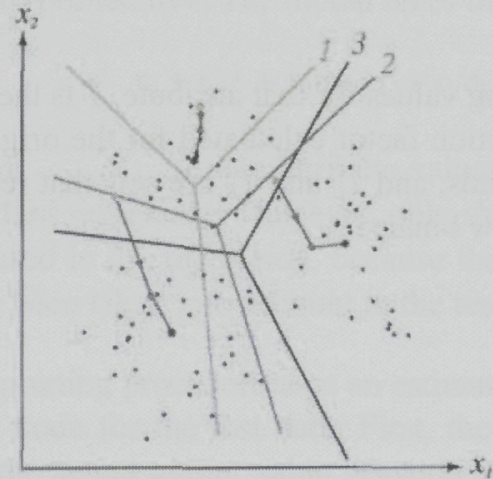

Figure 5.6. Dynamics of clustering by $k$ means. Seeds (three initial cluster centers) are introduced and the means change in successive iterations by three times.

\section{2) C-Fuzzy Clustering}

In the considered $k$-means procedure, each data point is assumed to be in exactly one cluster. We can relax this condition and allow each instance to belong to a cluster with some degree; that introduces a "fuzzy" membership in a cluster, so that a point may belong to several clusters, each with some degree in the range $[0,1]$ (Solomatine, 2005). This idea is used in the fuzzy C-means clustering algorithm (Bezdek, 1981).

It is based on the minimization of an objective function $J_{m}$, with respect to $U$, a fuzzy $c$ partition of the data set, and to $V$, a set of $K$ prototypes:

$$
J_{m}(U, V)=\sum_{j=1}^{n} \sum_{i=1}^{c} u_{i j}^{m}\left\|X_{j}-V_{i}\right\|^{2}, 1 \leq m<\infty
$$

where $m$ is any real number greater than $1, u_{i j}$ is the degree of membership of $X_{j}$ in the cluster $i, X_{j}$ is the $j^{\text {th }}$ component of the $d$-dimensional measured data, $V_{i}$ is the $d$ - 
dimensional center of the cluster, and $\|*\|$ is any norm expressed the similarity between any measured data and center.

Fuzzy partition is carried out through an iterative optimization of Equation (5.13) with the update of the membership $u_{i j}$ and the cluster centers $V_{i}$ by:

$$
\begin{gathered}
u_{i j}=\frac{1}{\sum_{k=1}^{c}\left(\frac{d_{i j}}{d_{i k}}\right)^{\frac{2}{m-1}}} \\
V_{i}=\frac{\sum_{j=1}^{n} u_{i j}^{m} X_{j}}{\sum_{k=1}^{n} u_{i j}^{m}}
\end{gathered}
$$

The iteration will stop when $\max _{i j}\left[\left|u_{i j}-\hat{u}_{i j}\right|\right]<\varepsilon$, which is a termination criterion between 0 and 1.

If such a loosening of the original clustering problem is feasible, this algorithm often produces a more robust clustering that is less susceptive to introduction of new (maybe extreme) points, however without clear-cut membership of instances in a particular cluster (Solomatine, 2005).

\subsubsection{Partial Least Square Regression}

The PLSR is a relatively recent technique that generalizes and combines features from principal component analysis and multivariate linear regression (Abdi, 2003). Instead of finding the hyperplanes of the minimum variance, it finds a linear model describing some predicted variables in terms of other observable variables.

A goal of PLSR (or, more precisely, of the PCA part in it) is to find orthogonal linear combinations of original predictors that correlate highly with the response variables, while accounting for as much variance in the predictors as possible. This means that the PLSR method balances the objectives of finding latent vectors that explain both the response and predictor variation. Therefore, the PLSR method is well suited to the prediction of regression models when the data is highly correlated, and where there is only a limited number of observations, because the predictor and predicted (response) variables are each considered as a block of variables (Rosipal and Krämer, 2006).

The PLSR models the relationship between the response and predictor variables by means of latent variables. For a data set with a response variable $y \in R^{n \times m}$ and a predictor variable $x \in R^{n x p}$, the PLSR decomposes the variables $x$ and $y$ as follows. 


$$
\begin{aligned}
& X=T P^{T}+E=\sum_{h=1}^{a} t_{h} p_{h}{ }^{T}+E \\
& Y=U Q^{T}+F=\sum_{h=1}^{a} u_{h} q_{h}^{T}+F
\end{aligned}
$$

where $T$ and $U$ are matrices of the extracted score vectors (components, latent vectors), $P$ and $Q$ represent matrices of the loadings, and $E$ and $F$ are the matrices of the residuals.

The input and output variables are projected onto a subspace of orthogonal latent variables to give the input and output scores $t$ and $u$, respectively. The standard algorithm for computing PLS regression components is the nonlinear iterative PLS (NIPLS). The NIPLS algorithm starts with a random initialization of the $Y$ score vector $u$, and repeats the sequence of steps below until convergence is achieved. After convergence, the loading vectors $p$ and $q$ can be computed. In summary, the NIPLS algorithm is as follows:

(1) set the output scores u equal to a column of $Y$

(2) compute the input weights $w$ by regressing $X$ on $u: w^{T}=\left(u^{T} \cdot X\right) /\left(u^{T} \cdot u\right)$

(3) normalize $\mathrm{w}$ to unit length: $w=w /(\|w\|)$

(4) calculate the input scores $t: t=(X \cdot w) /\left(w^{T} \cdot w\right)$

(5) compute the output loadings $q$ by regressing $Y$ on $t: q^{T}=\left(t^{T} \cdot Y\right) /\left(t^{T} \cdot t\right)$

(6) normalize $q$ to unit length: $q=q /(\|q\|)$

(7) calculate the new output scores $u: u=(Y \cdot q) /\left(q^{T} \cdot q\right)$

(8) check the convergence on $u$ - if 'yes' go to (9) or otherwise go to (2)

(9) calculate the input loadings $p$ by regressing $X$ on $t: p^{T}=\left(t^{T} \cdot X\right) /\left(t^{T} \cdot t\right)$

(10) compute the inner model regression coefficient $b: b=\left(t^{T} \cdot u\right) /\left(t^{T} \cdot t\right)$

(11) calculate the input residual matrix: $E=X-t \cdot p^{T}$

(12) calculate the output residual matrix: $F=Y-b \cdot t \cdot q^{T}$

(13) if additional PLS dimensions are necessary, replace $X$ and $Y$ by $E$ and $F$, respectively and repeat steps (1)-(13).

In the model prediction step, the prediction $\hat{Y}$ is calculated by the new input $\hat{X}$, and $p^{T}, q^{T}, W^{T}$ and $b$ are calculated in the model construction step.

\subsection{Application of DDM to Eco-environment}

Data is a collection of organized information, usually the result of experience, observation or experiment. This may consist of numbers, words, or images, particularly 
as measurements or observations of a set of variables in the natural environment. As it is obvious that data have specific characteristics in each domain, therefore, how much data should be used, what variables should be included and how long are the sampling intervals, are all important pieces of information for researchers. The reason is that data analysis methods also vary widely according to data availability and disciplines.

Environmental data have the characteristics of seasonal variations, various forcing factors, time lag between impacts on the environment and its responses, and similarities between neighboring sampling stations. These characteristics are different from data obtained for electro-machines and equipment representing noise. Therefore in selecting or developing a DDM, one has to scrutinize the machine learning (ML) algorithm, which is at the heart of data-driven models. When data are grouped by ML, the most important rule is if it considers relations between instances or/and variables.

In MTs, the SDR rule is a kind of classification. Such a classification rule is also explicit in cases of small numbers of attributes, for example, a stage-discharge rating-curve. Otherwise, in cases of large numbers of attributes, which impact on each other due to the many environmental factors, the binary split rule is not explicit any more, but it becomes implicit and generates a black box model. As described in the Chapter 2.3, eutrophication processes showing complex mechanisms due to many forcing function, bio-chemical reactions, and geo-morphological conditions, may be a typical case. In addition, the problem of the overall tree optimization can be computationally costly since each attribute should be tested across a number of possible split values (Bhattacharya and Solomatine, 2005; Bhattacharya and Solomatine, 2006; Slolmatine and Siek, 2006).

The eco-environmental data set, which includes biological processes always, goes through an irregular variation. Therefore modelling using ANNs and $k \mathrm{NN}$ inevitably may have a limitations for high dimensional eco-environmental data. As ANNs and $k \mathrm{NN}$ are effective in the case when a few variables have strong relationships each other, most studies have examined simple relationships between Chl-a and DO or $\mathrm{pH}$ (Schladow and Hamilton, 1996; Heiskary and Markus, 2001) for use in algal prediction models. The reason for this is that $\mathrm{DO}$ and $\mathrm{pH}$ are directly dependent variables for algal growth and decay, and can be easily measured.

To alleviate these problems, a clustering is proposed, which makes it possible to split the input space into several subspaces by taking all input and output attributes rather than an input attribute. More specifically, the subspace (each cluster center) is determined by a clustering method considering all input and output variables. Then, linear models are constructed in each subspace at the internal nodes. The expansion of an internal node is determined by comparing errors calculated at the parent node with the sum of those at the child nodes.

As discussed in Section 5.2.1.3), MTs are tree-structured regression models that associate leaves with multiple linear regression functions, which are used to calculate 
numerical values. Therefore a model tree constructs piecewise linear models at the leaves, but overall it shows a non-linear behaviour. On the other hand, when the number of observations is small compared with the number of predictors, a local model with a regression approach is no longer feasible. In particular, in the clustering technique with the model tree approach such as M5', one is often faced with this problem since the number of observations in the local model is decreased according to the expansion of the tree. Partial least square (PLS) regression is an extension of the multivariate linear regression to solve this problem by considering in-out pairs remaining at each internal node and at the final leaf. Specifically, PLS regression is effective in situations where the use of the traditional LSE based multivariate method is severely limited because there are fewer observations than the number of variables (attributes) (Tan et al., 2004; Baffi, 1999). 


\title{
Chapter 6
}

\section{CASE STUDY: DATA ACQUISITION \& ASSSESSMENT}

\section{Summary}

This chapter describes the physico-chemical and ecological features of the Yongdam reservoir. Flow rate, meteorological conditions and water quality were monitored over three years, 2005-2007 at ten sampling stations in the reservoir as well as five tributaries. The research site Yongdam reservoir is located in the upper region of the Geum river basin flowing through the mid-west of the Korean Peninsula that belongs to the temperate zone. The Yongdam reservoir has particular features such as a dendritic narrow, deep and long shape, thermal stratification, turbid density flows, and intake facilities for surface water. The retention time of the reservoir amounts to 318 days. About $70 \%$ of the annual rainfall is concentrated during the period of the summer monsoon from June to September; a main reason for the reservoir construction has been flood control and water storage for the dry season. In this study, physico-chemical and ecological parameters monitored at three depths in the water column identify double thermoclines of the reservoir water body and turbid density flows due to density differences between inflowing water and the receiving water body. Analyses of nutrients and eutrophication status show that the Yongdam reservoir is at the threshold of a limnological evolution, viz. through aging processes; thus highly increased nutrient and phytoplankton contents were found in 2005, i.e. only 3 years after filling the reservoir. The COD and phosphorus loadings etc. have been decreasing over the three years 2005-2007. The levels of pollutants in the upper reservoir section are generally higher than for the upstream sections. The middle layer, due to density flows during thermal stratification, shows an opposite results compared to the surface layer. This means that the water body of the Yongdam reservoir has been responding to the watershed with a complex mechanism. According to the Algal Growth Potential Test (AGPT), the Yongdam reservoir is a phosphate-limited water body; there may be inhibitors that hinder the algal growth in case of sufficient bio-available limiting nutrients existing in the water column. The lacustrine zone in the lower reach gives a better condition for algal growth because of a smaller turbulent length scale than that in the riverine and transition zones. The AGPT also identifies a mixing pattern of nutrients and the reservoir zones through their longitudinal and vertical AGP distributions. The surface water discharge of the Yongdam reservoir induces a transfer of pollutants from the middle layer to the surface layer. This nutrient transportation mechanism reduces nutrient accumulation at the bottom of the reservoir, but it also offers an opportunity for continuous algal production during thermal stratification of the surface water.
\end{abstract}

\subsection{Research Site}




\subsubsection{Geographic and Limnological Features}

South Korea is geo-morphologically characterized by many hills and mountains, which occupy nearly $70 \%$ of its territory. Low hills are mostly located in the south and the west of the country, and give way gradually to increasingly higher mountains towards the north and the east. On the whole, the western and the southern slopes of the Korean Peninsula are gentle with various types of plains, low hills, and basins developing along the rivers.

This study was carried out in the upper region of the Geum river basin where the Yongdam reservoir is located. The Geum river flows through the mid-west of the Korean peninsula over $36^{\circ} 00^{\prime} \sim 35^{\circ} 35^{\prime} \mathrm{NL}, 127^{\circ} 20^{\prime} \sim 127^{\circ} 45^{\prime} \mathrm{EL}$; the area belongs to the temperate region having four seasons (see Figure 6.1). The Geum river basin has a drainage area $9,810 \mathrm{~km}^{2}$ and a mainstream length of $396 \mathrm{~km}$. The Geum river basin is one of four major river basins in South Korea, where other river basins are the Han, the Nakdong, and the Seomjin river basins (see Figure 6.1).
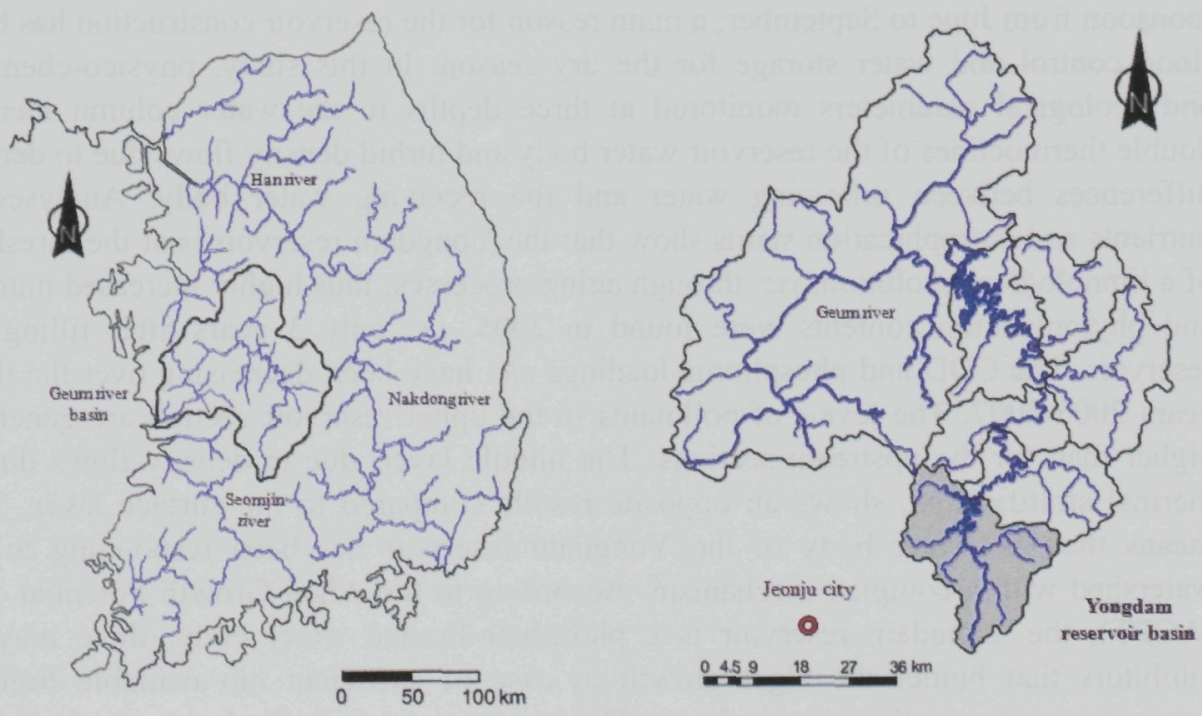

Figure 6.1. Four major river basins in South Korea (a); Location of the Yongdam reservoir in the Geum river basin (b).

The Yongdam reservoir was constructed for several purposes such as flood control, domestic water supply and power generation. The basin of the Yongdam reservoir has a catchment area of about $930 \mathrm{~km}^{2}$ and consists of forests $\left(725 \mathrm{~km}^{2}\right.$, about $\left.73 \%\right)$, farming area $\left(139 \mathrm{~km}^{2}\right.$, about $\left.15 \%\right)$ and other lands $\left(65 \mathrm{~km}^{2}, 12 \%\right)$ including the reservoir water surface, and streams (see Figure 6.2).

The Yongdam reservoir is a narrow, deep reservoir with an area of about $36 \mathrm{~km}^{2}$ in surface, about one kilometer in width, and with a maximum depth of about $70 \mathrm{~m}$. The 
reservoir has a retention time $\left(t_{d}=V / Q\right)$ of 318 days. The reservoir, located in a temperate monsoon area undergoes strong thermal stratification twice a year. The characteristics of the Yongdam reservoir are listed in Table 6.1.

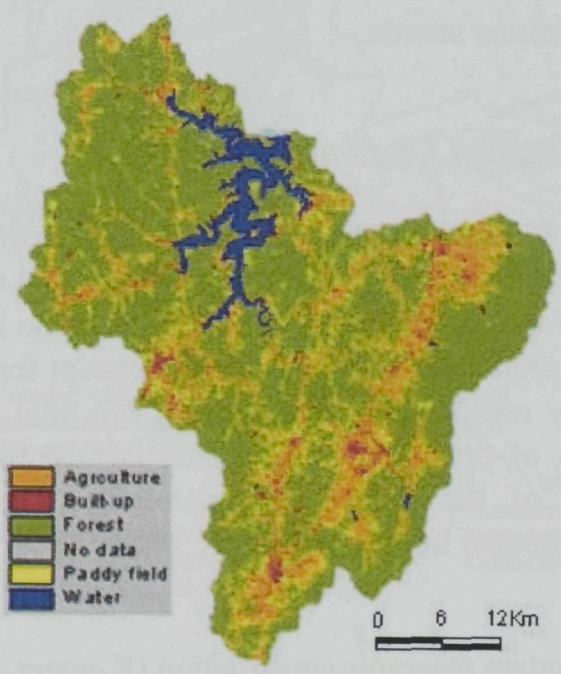

Figure 6.2. Land use for the Yongdam reservoir basin in December 2007 (Kwater, 2007)

Table 6.1. General geographic and limnological features of the Yongdam reservoir.

\begin{tabular}{ll}
\hline \multicolumn{1}{c}{ Attributes } & \multicolumn{1}{c}{ Yongdam reservoir } \\
\hline Latitude / Longitude & $36^{\circ} 00^{\prime}-35^{\circ} 35^{\prime} \mathrm{N} / 127^{\circ} 20^{\prime}-$ \\
& $127^{\circ} 45^{\prime} \mathrm{E}$ \\
Dam Elevation $(\mathrm{EL}, \mathrm{m})$ & 268.5 \\
Project period of dam construction & October 1996 - November 2001 \\
Impoundment of reservoir & 30 October 2001 \\
Functions & Multipurpose reservoir \\
- Water supply / Flood control $\left(\times 10^{6} \mathrm{~m}^{3}\right)$ & $650 / 137$ \\
- Hydroelectric power generation $(\mathrm{kW})$ & 24,400 \\
Reservoir shape type & Dendritic \\
Circulation pattern & Polymictic, river-reservoir system \\
Trophic state & Meso-eutrophic \\
Average rainfall (mm/year $)$ & 1,260 \\
Average inflow / outflow $\left(\mathrm{m}^{3} / \mathrm{s}\right)$ & $24.4 / 20.6$ \\
Watershed /Reservoir surface area* $\left(\mathrm{km}^{2}\right)$ & $930 / 36.2$ \\
Reservoir shoreline* / Max. width* $\left(\mathrm{km}^{*}\right)$ & $50 / 1$ \\
Maximum depth* $(\mathrm{m})$ & 70 \\
Reservoir storage volume* $\left(\times 10^{6} \mathrm{~m}^{3}\right)$ & 815 \\
Effective storage volume $\left(\times 10^{6} \mathrm{~m}^{3}\right)$ & 672 \\
Hydraulic retention time $($ days $)$ & 318 \\
Dam height / length $(\mathrm{m})$ & $70 / 498$ \\
\hline
\end{tabular}

Source: Design Report of the Yongdam reservoir (K-water, 1991)

*At normal high water level

The construction of the Yongdam reservoir has changed the ecosystem of the Geum river and the surrounding areas. Generally, aquatic ecosystems are influenced by flow 
conditions. A dam construction may directly be linked to changes in flow conditions, which are affected by geomorphologic features of the reservoir: length, depth, area (both of the water surface and of the drainage area,) and hydraulic retention time. Especially, deep reservoirs are not well mixed and are mostly stratified by temperature gradients in the water column during the summer season.

\subsubsection{Hydrologic and Meteorological Characteristics}

The Korean Peninsula has two monsoons. The summer monsoon brings abundant moisture from the ocean and produces heavy rainfall. About $70 \%$ of the annual rainfall occurs from June to September. The winter monsoon, which originates from the interior of the Asian continent, is dry and cold. It produces little precipitation except for a few winter snowfalls. Winter months normally receive less than $10 \%$ of the total annual precipitation. This means that the pattern of precipitation in Korea is unstable both seasonally and spatially due to effects of the monsoon climate. The mean air temperature of the Geum river during the hottest month of June is $34.1^{\circ} \mathrm{C}$ and during the coldest month of January $-4.9^{\circ} \mathrm{C}$.

According to the design report for the Yongdam reservoir construction (K-water, 1991), in the catchment area of the Yongdam reservoir the yearly mean precipitation amounts to about $1,200 \mathrm{~mm}$, and the evaporation is $1,050 \mathrm{~mm}$ a year. The monthly average runoff in July $\left(77 \mathrm{~m}^{3} / \mathrm{sec}\right)$ is about 10 times higher than the average runoff in January $\left(7.8 \mathrm{~m}^{3} / \mathrm{sec}\right.$ ) because of seasonal rainfall. The average runoff is $24.4 \mathrm{~m}^{3} / \mathrm{sec}$, and the minimum flow is only $1.2 \mathrm{~m}^{3} / \mathrm{sec}$. The annual mean water temperature amounts to 11.4 ${ }^{\circ} \mathrm{C}$ with monthly mean temperatures $25.1{ }^{\circ} \mathrm{C}$ in August and the surface water frozen in the upper reservoir section in January.

\subsubsection{Pollution Sources}

In the Yongdam reservoir basin the main pollution sources come from human activities and livestock farming. The population in the Yongdam reservoir basin area has increased from 45,000 in 2005 to 49,000 in 2008 and is distributed over five tributary catchments: T1-T5 with the largest population in catchment T2 (see Figure 6.3). In general, land use in rural areas is a primary indicator for the identification of pollution sources, whether they are point or non-point sources, together with the pollution production by pork and poultry.

Table 6.2 presents the different pollution sources, the total production values of BOD, total nitrogen and total phosphorus, as well as loadings of these pollutants to the surface water of the Yongdam reservoir basin (K-water, 2002). In the basin, three wastewater treatment plants are operated (see Figure 6.3). The total capacity of the treatment plants is $7,000 \mathrm{~m}^{3} /$ day, 52,000 population equivalents. In addition to these plants, 48 small village sewerage systems are being operated. The problem, however, is that all wastewater treatment processes under operation are poor in removing phosphorus. 


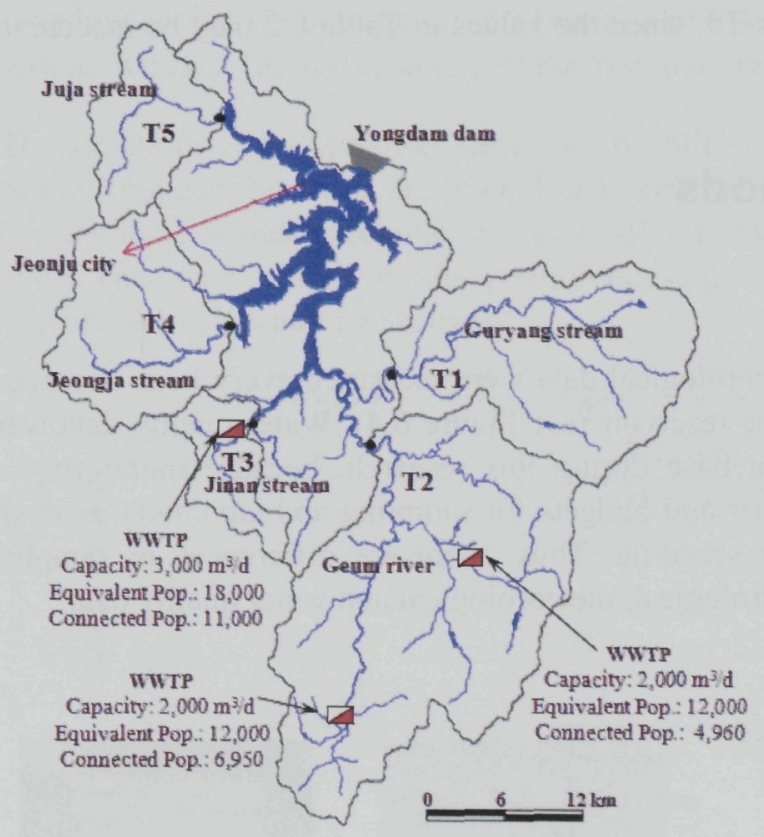

Figure 6.3. Yongdam reservoir basin with five tributaries (T1-T5). Also indicated are the locations of the tributary monitoring stations and the wastewater treatment plants.

Table 6.2. Contaminant source, production and load of the Yongdam reservoir basin in 2002 (a) Production and loadings classified by sources

\begin{tabular}{c|c|c|c|c|c|c|c|c|c|c}
\hline \multirow{2}{*}{ Nutrients } & \multicolumn{2}{|c|}{ Population } & \multicolumn{2}{c|}{ Livestock } & \multicolumn{2}{c|}{ Land use } & \multicolumn{2}{c|}{ Industrial } & \multicolumn{2}{c}{ Total } \\
\cline { 2 - 12 } & Prod. & Load. & Prod. & Load. & Prod. & Load. & Prod. & Load. & Prod. & Load. \\
\hline BOD (kg/day) & 2,358 & 1,787 & $\begin{array}{c}14,48 \\
6\end{array}$ & 156 & 1,672 & 418 & 88 & 87 & $\begin{array}{c}18,60 \\
3\end{array}$ & 2,449 \\
\hline T-N (kg/day) & 593 & 574 & 3,389 & 268 & 2,787 & 697 & 66 & 66 & 6,835 & 1,604 \\
\hline T-P (kg/day) & 68 & 67 & 1,218 & 29 & 181 & 46 & 9 & 9 & 1,476 & 150 \\
\hline
\end{tabular}

Source: Nutrient production in the Yongdam reservoir basin and loadings (K-water, 2002).

(b) Production and loadings classified by five tributary catchments

\begin{tabular}{c|c|c|c|c|c|c|c|c}
\hline \multirow{2}{*}{ Items } & \multicolumn{2}{|c|}{ T1 \& T2 } & \multicolumn{2}{c|}{ T3 } & \multicolumn{2}{c|}{ T4 \& T5 } & \multicolumn{2}{c}{ Total } \\
\cline { 2 - 9 } & Prod. & Load. & Prod. & Load. & Prod. & Load. & Prod. & Load. \\
\hline TN $(\mathrm{kg} /$ day $)$ & 8,161 & 4,106 & 3,714 & 2,724 & 2,906 & 1,590 & 14,781 & 8,420 \\
\hline TP $(\mathrm{kg} /$ day $)$ & 1,315 & 168 & 392 & 95 & 304 & 65 & 2,011 & 328 \\
\hline
\end{tabular}

Source: Nutrient production in the Yongdam reservoir basin and loadings (K-water, 2002).

According to Table 6.2 (a), the total loadings of phosphorus and nitrogen from domestic wastewater reached about $44 \%(67 / 150)$ and $36 \%(574 / 1,604)$ of the total, respectively. Although the livestock farming has the largest phosphorus production $(1,218 \mathrm{~kg} / \mathrm{day})$, the load ( $29 \mathrm{~kg}$ /day) is low. The reason may be that most livestock are bred by pasturing, and only a few barns were counted for calculation of loadings. A research on a basin level should be practiced to evaluate contaminant sources, their loadings and impacts on 
the reservoir in more detail for T1-T5, since the values in Table 6.2 may be inaccurate, especially the land use values.

\subsection{Material and Methods}

\subsubsection{Monitoring}

In this study, hydrologic and meteorological data were measured every hour and every three hours, respectively across the reservoir (see Figure 6.4). Water quality data were collected via another planned database during this research, because monitoring of water quality, which requires efforts and budgets for sampling and laboratory analysis, is generally limited to a routine schedule. Thus, there are differences in sampling frequency for the data groups: hydrological, meteorological and water quality data.
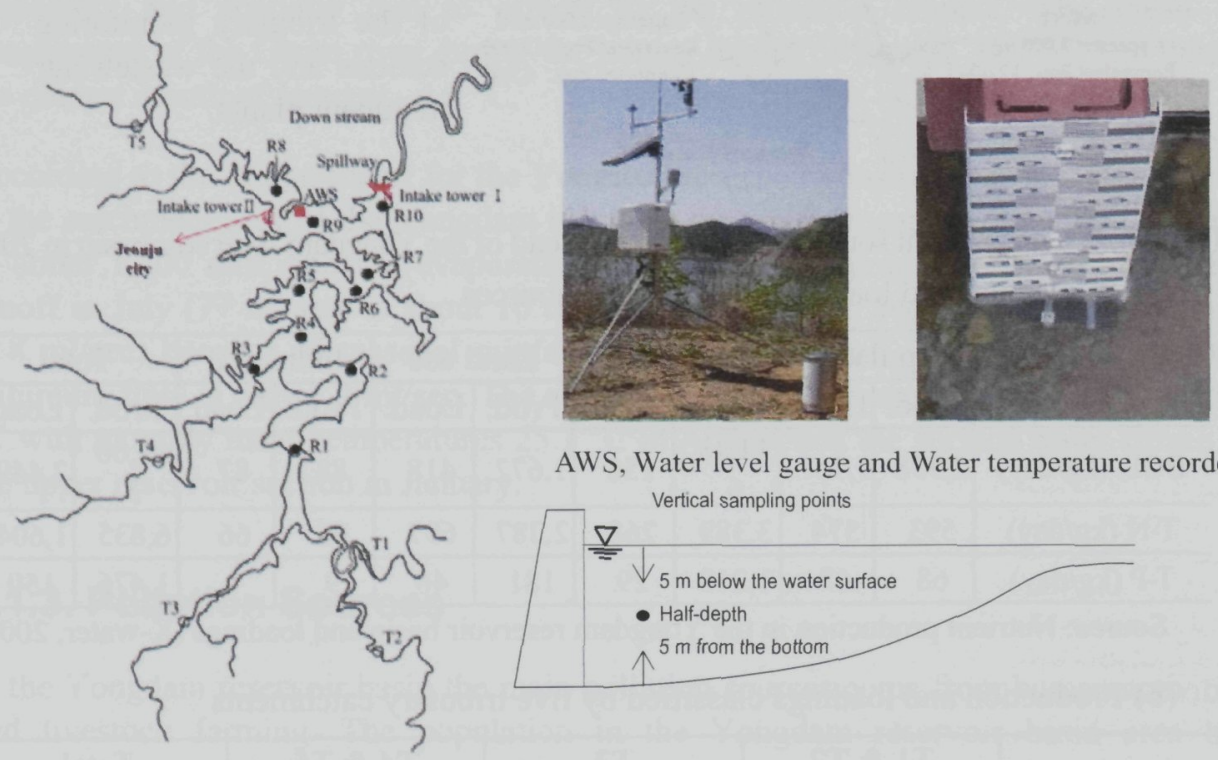

AWS, Water level gauge and Water temperature recorder

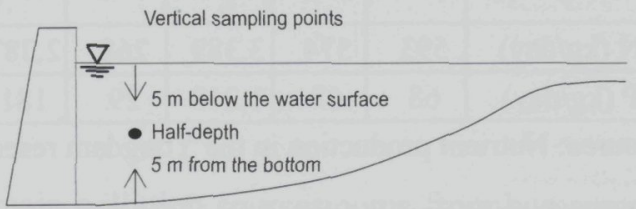

Figure 6.4. Water quality monitoring stations in the reservoir (R1-R10) at the three vertical points. The photographs show the set-up of the automatic weather station installed in the reservoir, water level gauge and automatic water temperature recorder

\subsubsection{Hydrological and Meteorological Measurements}

The hydrological data have been accumulated in one-hour intervals at five monitoring stations for inflow (T1-T5) and outflow (Intake I \& II, Spillway) as shown in Figure 6.4. The parameters of meteorological data were: air temperature, humidity, wind direction, wind speed, dew point, and cloudiness over every three hours. To acquire meteorological data, an AWS (automatic weather station) was installed near the 
sampling station R8 in the reservoir (see Figure 6.4). The data from the Jeonju weather station, which is located upstream of the Yongdam reservoir, were also acquired.

The variations of precipitation, flow rates of inflow and outflow, as well as water levels of the Yongdam reservoir are plotted in Figure 6.5. As shown in Table 6.3 and Figure 6.5 , the mean annual precipitation was $1,445 \mathrm{~mm}$ over the 3 -year periods from 2005 to 2007. The mean rainfall on precipitation days and the corresponding ranges were 10.0 $\mathrm{mm}$ and $0.1 \sim 183 \mathrm{~mm}$ respectively.
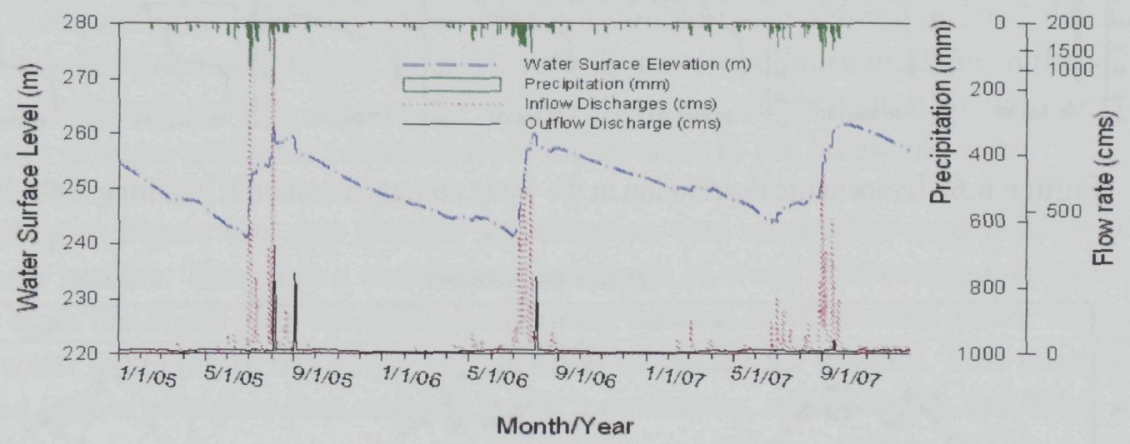

Figure 6.5. Hydrological parameters for the Yongdam reservoir from 2005 to 2007.

The mean inflow to the reservoir and the range of the discharges were $23.9 \mathrm{~m}^{3} / \mathrm{s}$ and 0.1 $\sim 1,980 \mathrm{~m}^{3} / \mathrm{s}$, and the mean outflow from the reservoir and the range of discharges were $23.4 \mathrm{~m}^{3} / \mathrm{s}$ and $9.1 \sim 706 \mathrm{~m}^{3} / \mathrm{s}$, respectively. These flow rate data are quite higher than those of the data before the construction of the Yongdam reservoir. Consequently, it can be seen that the Yongdam reservoir has complicated reservoir management conditions because the precipitation and runoff are concentrated during the summer season; they will change the whole reservoir ecosystem every year.

Table 6.3. Monthly average precipitation and inflow of the Yongdam reservoir (from 2005 to 2007)

\begin{tabular}{cccccccccccccc}
\hline Items & Jan & Feb & Mar & Apr & May & Jun & Jul & Aug & Sep & Oct & Nov & Dec & Sum/mean \\
\hline $\begin{array}{c}\text { Precipitation } \\
(\mathrm{mm})\end{array}$ & 15.7 & 44.5 & 72.9 & 60.1 & 103 & 142 & 464 & 273 & 191 & 27.5 & 33.2 & 30.1 & 1455.5 \\
Inflow $(\mathrm{m} 3 / \mathrm{s})$ & 1.7 & 6.0 & 9.5 & 9.9 & 10.1 & 11.1 & 116.3 & 61.2 & 53.3 & 3.9 & 1.8 & 1.6 & 24.1 \\
\hline
\end{tabular}

\subsubsection{Water Temperature and Thermal Stratification}

The Yongdam reservoir experiences a strong thermal stratification from late spring to late autumn (i.e. April to October; see Figure 6.6 and 6.7). In early April, the epilimnion (surface layer) is shallow, but it deepens with the rise of air temperature, and finally spreads over the whole reservoir due to destratification processes with cooling of the surface water by lowering of the air temperature. 


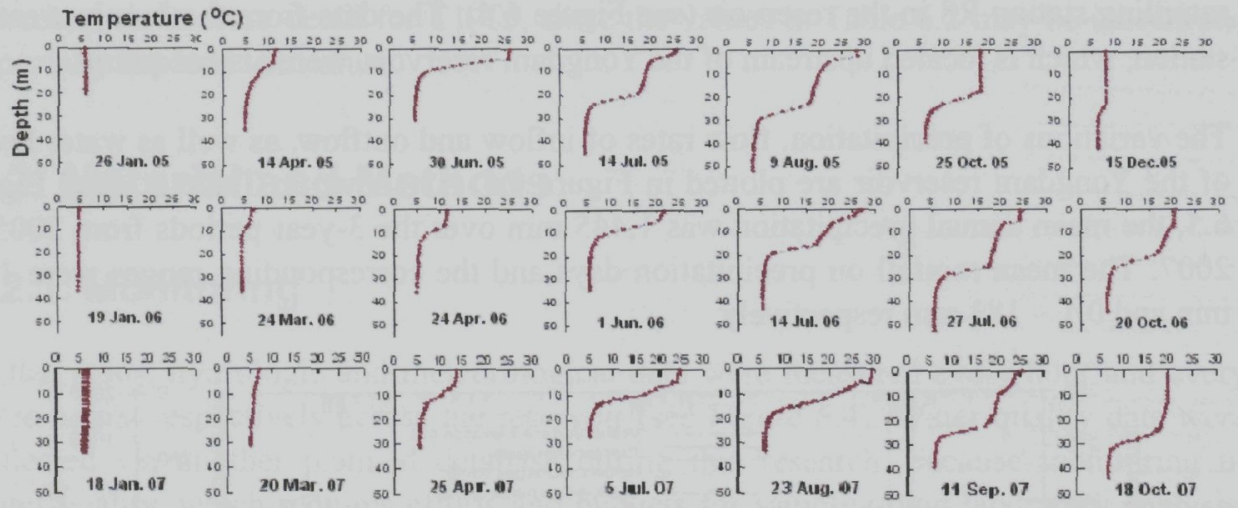

Figure 6.6. Temperature distribution in the water column at station R10 during 2005-2007.
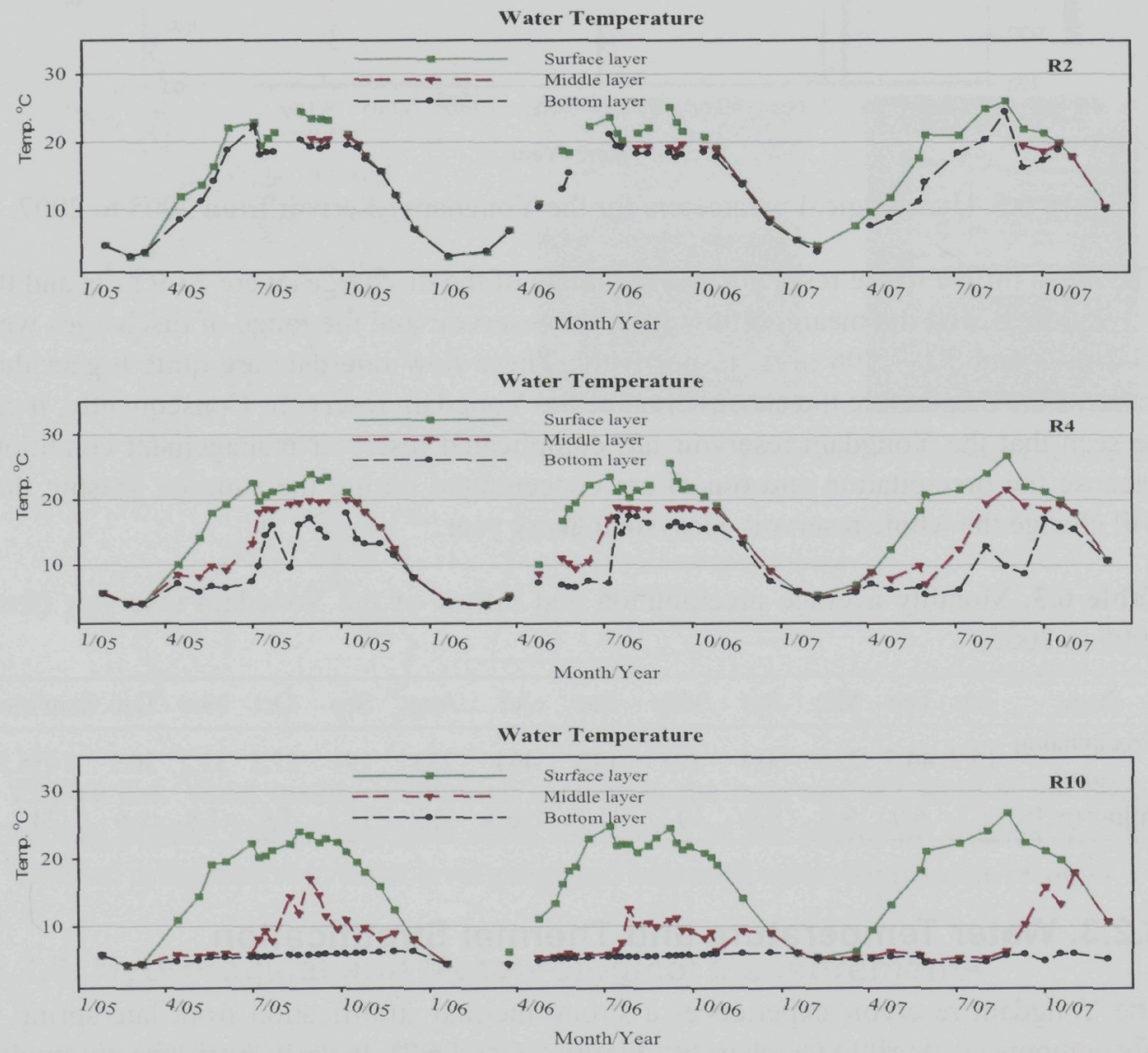

Figure 6.7. Temperature distribution in the water column at R2 (riverine zone, mean depth: 20m), R4 (transition zone, mean depth: $25 \mathrm{~m}$ ) and R10 (lacustrine zone, mean depth: $43 \mathrm{~m}$ ) for the three years (2005-2007). The missing points at R2 represent unavailable access for sampling due to flood flow and low water level. 
The mean water temperature in the surface layer was $17.0^{\circ} \mathrm{C}$ over the three years 2005 2007 (max.: $26.9^{\circ} \mathrm{C}$; Min.: $0^{\circ} \mathrm{C}$ ). To give a few examples in the middle and bottom layer of station R4 (transition zone), the water temperatures vary according to the water depths. The station R2 (riverine zone) shows temporal thermoclines in summer due to a deeper water depth. In the bottom layer of station R10 (lacustrine zone), the water temperature was found to be average on $5.5^{\circ} \mathrm{C}$ over the whole year regardless of air temperature.

Double thermoclines were often observed during 2005 and 2006. These double thermoclines probably take place due to flood flows which strongly affect the interflow in the water column. However, there were not double thermoclines in 2007 due to the long monsoon flood (see Figure 6.5). Therefore, the temperature gradient in the surface layer is relatively gentle. Figure 6.7 shows that the thermal stratification becomes gradually stronger along the reservoir from the riverine to the lacustrine zone.

According to Figure 6.6, this thermal stratification was reached at around $25 \mathrm{~m}$ below the water surface. Therefore a circulation (exchange of water) between upper and lower layers from the depth does not take place during thermal stratification. There was also a steep water temperature rise in the bottom layer of station R4 in 2006. This must be due to flood inflow of higher than ambient water temperature, which shows that R4 is at the transition zone between riverine and lacustrine conditions. Station R2 is a typical riverine zone showing similar patterns in temperature perturbation in all layers.

\subsubsection{Basic Water Quality Parameters}

Water quality monitoring took place at the five tributaries T1-T5 (see Figure 6.3) and at ten stations, R1-R10 in the reservoir (see Figure 6.4) over three years viz. 2005-2007. Monitoring intervals were variable from monthly to weekly in order to consider water quality variations closely related to the seasons. Often sampling was not possible in case of storm floods, strong winds and frozen conditions in the upstream part of the reservoir. The total number of sampling rounds amounted to 60 over above three years.

Sampling in the reservoir was conducted at three vertical points ( $5 \mathrm{~m}$ below-the water surface, at half-depth, and $5 \mathrm{~m}$ above-the bottom). The water depth was measured in advance with a Hondex PS-7-digit sounder depth finder and then the water was sampled at three points by a Van Dorn sampler $(2,500 \mathrm{~mL})$.

The sampled water was immediately transferred to an aseptic polythene bottle $(2,000$ $\mathrm{mL}$ ), completely filled and then kept in a box before transportation to the laboratory. Here the samples were stored in a refrigerator at $4{ }^{\circ} \mathrm{C}$ before analysis.

Physico-chemical parameters analyzed in the field were: water temperature, dissolved oxygen (DO), $\mathrm{pH}$, electrical conductivity (EC) and turbidity (in NTU). These parameters were measured in situ using a multi-water quality measuring meter (Troll 9500). Water temperature for inflow water at the five tributaries (T1-T5), which is an important forcing function for physically-based modelling, was measured with a water- 
temperature recorder (HOBO Scientific Co., USA) in one-hour-interval readings. SD (Secchi-depth) was also measured to assess the eutrophication level on site.

The chemical (nutrient) parameters analyzed were: total nitrogen (TN), ammonianitrogen, nitrate-nitrogen and nitrite-nitrogen, total phosphorus (TP), inorganic phosphorus ( $\mathrm{PO}_{4}{ }^{3-}$ : soluble reactive phosphorus; SRP).

The following methods described in Standard Methods (APHA et al., 1995) were used for analysis of nutrients: Phenate Method for Ammonia, Colorimetric Method for Nitrite, Cadmium Reduction Method for Nitrate, and Ascorbic Acid Method for SRP. TN and TP were analyzed by the methods of Ultraviolet Spectrometric and Ultraviolet Spectrometric, respectively.

The chemical oxygen demand (COD) was measured with the potassium dichromate $\left(\mathrm{K}_{2} \mathrm{Cr}_{2} \mathrm{O}_{7}\right)$ destruction method for both the water and blank samples, and the two were compared. The oxygen demand in the blank sample was s subtracted from the COD for the original sample to ensure a true measurement of organic matter (APHA et al., 1995).

The fraction of labile dissolved organic carbon (LDOC), refractory dissolved organic carbon (RDOC), labile particulate organic carbon (LPOC) and refractory particulate organic carbon (RPOC) with respect to TOC were taken as follows: the ratios of DOC and POC to TOC as 0.466 and 0.534 , respectively. The other fractions with respect to TOC were: 0.093 (LDOC), 0.373 (RDOC), 0.162 (LPOC) and 0.372 (RPOC). These ratios were applied to the calculation of fractions of dissolved organic matter (DOM), which are the input variables for physically-based water quality modelling.

\subsubsection{Biological Parameters}

Biological parameters measured for this study are Chlorophyll-a (Chl-a) and algal growth potential (AGP). For in situ measurement of Chl-a, a fluoroprobe (BBE Co., Germany) was used, which is a highly sensitive measuring instrument for total chlorophyll analysis.

However in the case that in-situ measurement is impossible because of high turbid water, it was measured at the laboratory by the ESS method 150.1: ChlorophyllSpectrophotometric. Values were measured three times and then averaged. The AGP test (AGPT) described in the following section was carried out only in 2005 because of similar results and due to time and budget constraints.

\subsection{Water Quality Assessment}

\subsubsection{Basic Water Quality}

Here the results for the water quality parameters at stations representing reservoir zones are discussed according to the types of water quality issue. 


\section{1) Dissolved Oxygen (DO)}

Dissolved oxygen (DO) levels (see Figure 6.8) were generally above $5 \mathrm{mg} \mathrm{O} / \mathrm{L}$ in the surface layer over the three years (2005-2007) except for the flood seasons.
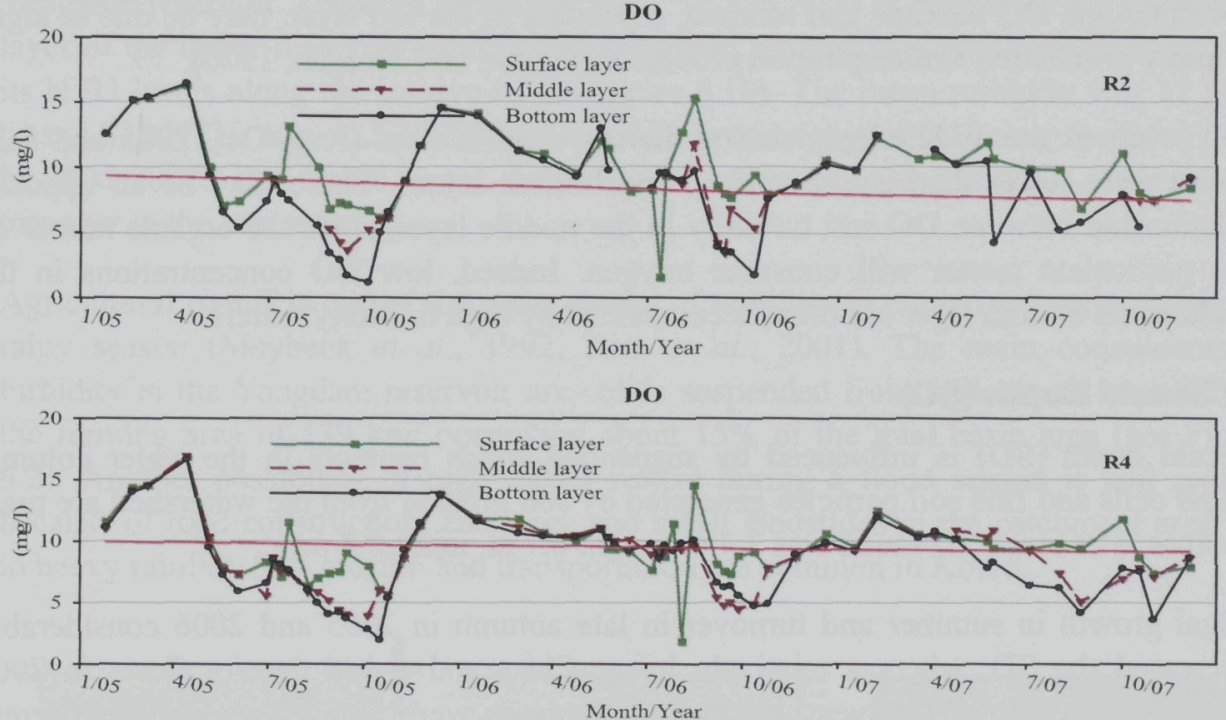

DO

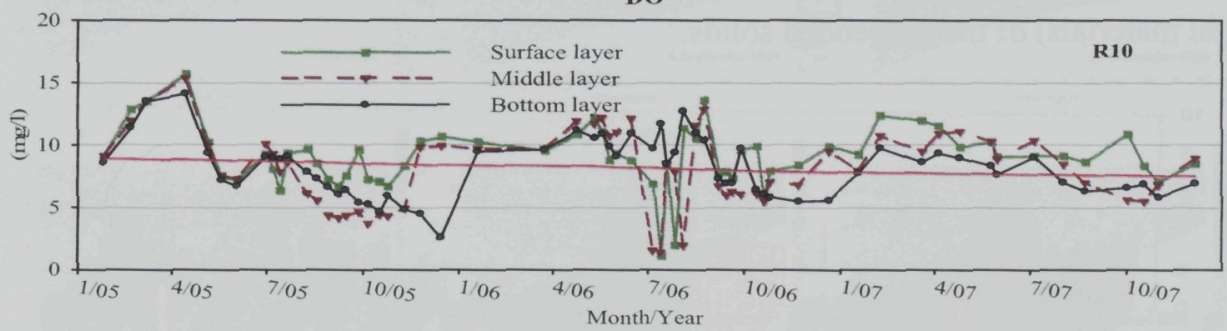

Figure 6.8. Distribution of DO concentration in the water column and trend in the bottom layer at R2 (riverine zone, mean depth: 20m), R4 (transition zone, mean depth: $25 \mathrm{~m}$ ) and R10 (lacustrine zone, mean depth: $43 \mathrm{~m}$ ) for the three years (2005-2007).

The mean DO levels were $9.5 \mathrm{mg} \mathrm{O} / \mathrm{L}$ (max.: 16.9, min.: 1.1) in the surface layer, $8 \mathrm{mg}$ $\mathrm{O}_{2} / \mathrm{L}$ (max.: 16.9 , min.: 1.4) in the middle layer and $8.3 \mathrm{mg} \mathrm{O}_{2} / \mathrm{L}(\max .: 16.6$, $\min .: 1.1)$ in the surface layer. In the middle layer of station R2 and R4, DO levels were found to be lower than in the surface layer during the summer flood season. This must be due to loadings of organic matter from the watershed and algal growth in the surface water. Above mean DO values satisfy the standard of $5 \mathrm{mg} \mathrm{O}_{2} / \mathrm{L}$ of the Korea Ministry of Environment (K MOE, 1987) and the DO standard of $5.5 \mathrm{mg} \mathrm{O}_{2} / \mathrm{L}$ as a 30 -day mean, as given by US EPA (1986). 
DO trends are similar to the water temperature variations with quite homogeneous DO levels over depth in station R2 and largest differences in station R10. DO concentrations gradually decrease in the bottom layer (see Figure 6.8). In the latter station relative low DO values in the bottom layer can be ascribed to hypolimnic and sediment DO consumption. DO maxima and minima, especially in the top layer, may be due to algae primary production and respiration processes (Stumm and Morgan, 1970).

DO levels in the middle layer during thermal stratification (Aug.-Oct. 2005 and June 2006) were lower than in the surface and bottom layers. There may be an opposite relationship between DO and turbidity in the middle layer, since the organic matter on the particulate matter will consume oxygen. Indeed, low DO concentrations in the middle and bottom layer are often accompanied by high turbidity waters.

\section{2) Secchi Depth (SD)}

Secchi depth (SD) is influenced by suspended solids contents in the water column. Algal cells and fine soil particles generated by soil erosion from the watershed are main sources. The mean SD found was $2.4 \mathrm{~m}$ (max.: $6.7 \mathrm{~m}$, min.: $0.3 \mathrm{~m}$ ).

Algal growth in summer and turnover in late autumn in 2005 and 2006 considerably decreased the SD, whereas relatively lower Chl-a and turbid density flows in 2007 increased the SD for that year. No clear differences were found between the different monitoring stations, probably because of the mixed origin (dead suspended matter vs. algal materials) of the suspended solids.

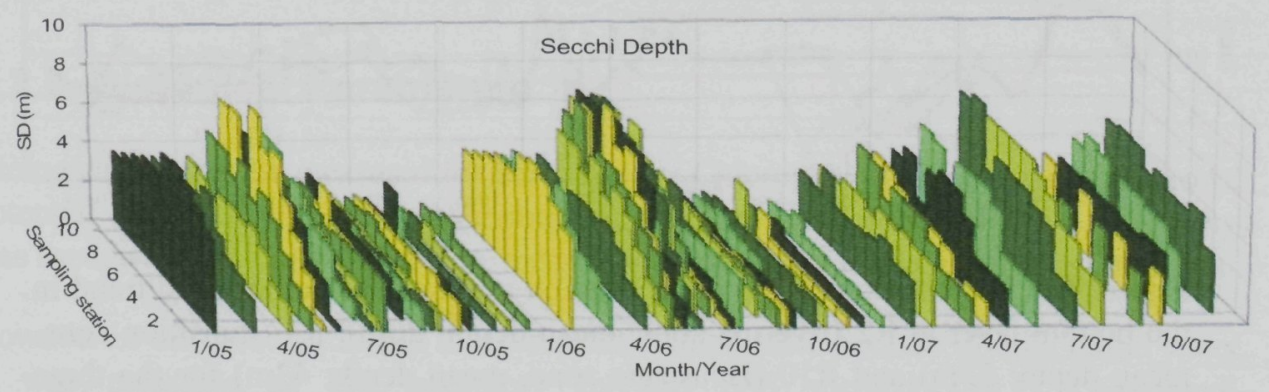

Figure 6.9. Secchi depth (SD) values for the three monitoring years (2005 2007).

According to Figure 6.9, the turbidity water during flood flow in summer does not affect the SD due to strong thermal stratification. Consequently, low SDs from the summer to winter seasons are mainly influenced by the algal growth in the surface layer and turnover of turbid water in the middle layer.

\section{3) Turbidity}

Figure 6.10 depicts the turbidity of the water flow along the reservoir. The often occurring density motions at intermittent water depth are due to different densities 
between inflowing river water and the receiving water body (Moore and Thornton, 1988; Basson, 1998).

Precipitation and stream flow are the transport media for the pollution sources to the Yongdam reservoir. The high turbidities (max.: 1,235 NTU at station R2) in the bottom layer of the upper reservoir sections is transported through the middle layer decreasing its NTU levels along the reservoir (see Figure 6.10). The mean turbidity was 32 NTU (max.: 519 NTU, min.: $0.5 \mathrm{NTU}$ ) in the middle layer of station R10 over the three years in 2005-2007. The turbid water current was preserved until just after the reservoir turnover in the late autumn.

Agricultural runoff is one of the main causes of turbidity in many reservoirs during the rainy season (Meybeck et al., 1992; Kim et al., 2001). The main constituents of turbidity in the Yongdam reservoir are solids suspended from agricultural runoff from the farming area of $139 \mathrm{~km}^{2}$ occupying about $15 \%$ of the total basin area (see Figure 6.2). Another possibility of high turbid waters during a flood season is soil erosion because of road construction, and large and small landslides in the catchment area due to heavy rainfall. Soil erosion and transportation are common in Korea.

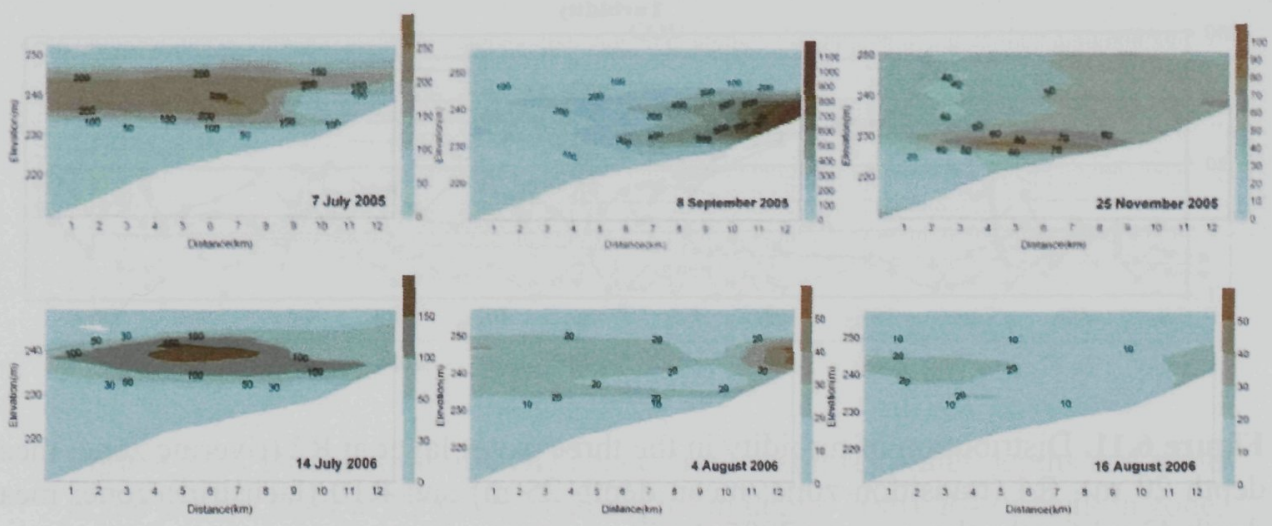

Figure 6.10. Turbid water currents in the Yongdam reservoir in 2005 and 2006

There was not turbidity flow in 2007 in spite of the presence of a thermocline due to gradual rainfall intensity. This will cause a reduction in the water temperature gap between stream inflows and the receiving water body.

The turbidity currents in the Yongdam reservoir are presented in more detail in Figure 6.11. In the riverine zone (R2) and transition zone (R4) high turbidity values were found in the middle and bottom layers during 2005 and 2006. Especially the highest turbidity values in the lacustrine zone (R10) took place in the middle layer during double thermoclines in 2005. In station R10, the highest turbidity in the bottom layer after 2005 indicates that the settling of soil particles takes place slowly and the bottom layer is not affected by the reservoir turnover. 

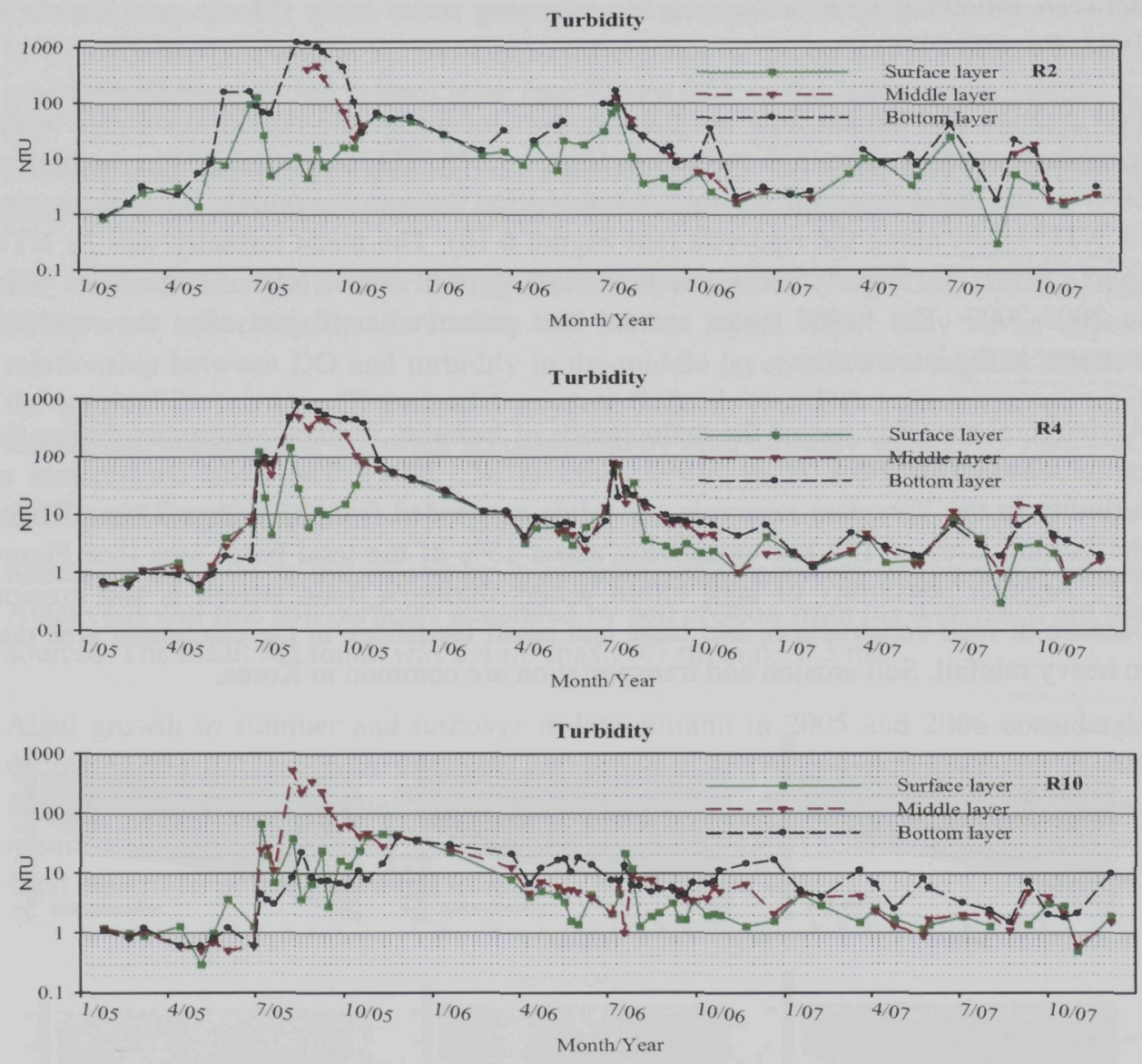

Figure 6.11. Distribution of turbidity in the three water layer at R2 (riverine zone; mean depth $20 \mathrm{~m}$ ), R4 (transition zone; mean depth $25 \mathrm{~m}$ ) and R10 (lacustrine zone; mean depth $43 \mathrm{~m}$ ) for the three years (2005-2007).

\section{4) Chemical Oxygen Demand (COD)}

The mean COD level was $2.8 \mathrm{mg} \mathrm{O}_{2} / \mathrm{L}$ in the entire reservoir (max.: 7.5 at station $\mathrm{R} 4$, min.: 0.7 at station $\mathrm{R} 4)$, which is the upper limit $\left(6 \mathrm{mg} \mathrm{O}_{2} / \mathrm{L}\right)$ of the raw water quality criteria for drinking water sources based on conventional drinking water treatment processes (K MOE, 1987). Figure 6.12 shows that Chemical oxygen demand (COD) was decreasing over the three years $(2005-2007)$. This decreasing trend may come especially from decreased loadings of pollutants from the watershed.

COD levels also follow seasonal variations due to nutrient loadings and their resultant algal growth in the summer season. In the surface layer of the lacustrine zone, the algal growth maybe contributes to the higher COD compared to the riverine zone. 

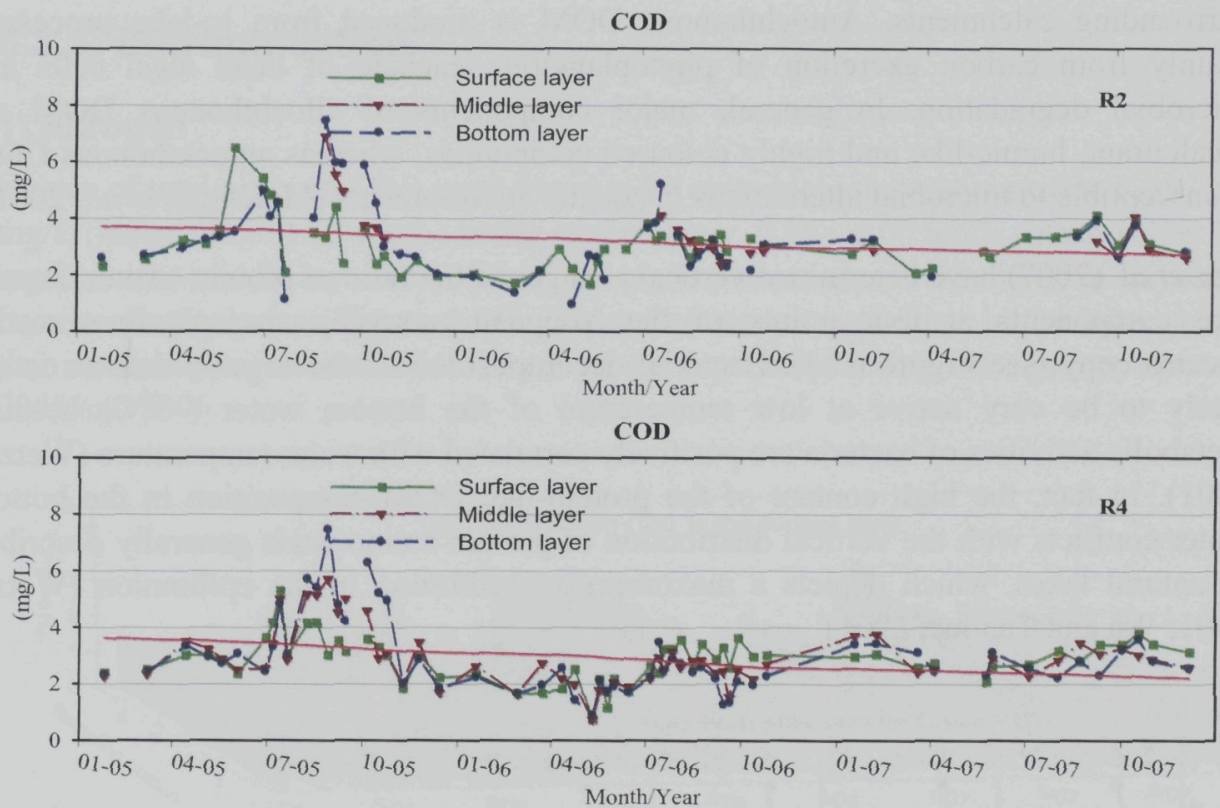

COD

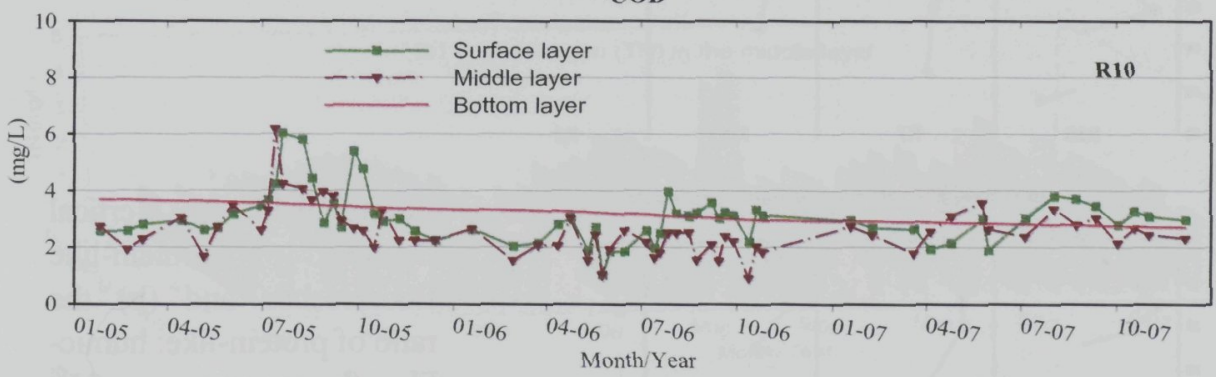

Figure 6.12. Distribution of COD concentration at three water depths and trend in the bottom layer at R2 (riverine zone, mean depth: 20m), R4 (transition zone, mean depth: $25 \mathrm{~m}$ ) and R10 (lacustrine zone, mean depth: $43 \mathrm{~m}$ ) for the three years (2005-2007).

\subsubsection{Organics}

The turbid interflow is known to supply massive quantities of organic carbon and nutrients together with fine solids to the reservoirs (Kim et al., 2000; Shin et al., 2004). Dissolved organic matter (DOM) is a heterogeneous mixture of constituents having a wide range of physico-chemical properties: carbohydrates, proteins, lignins, organic acids, natural polymers, etc. In drinking water treatment processes, DOM is known to be a precursor of chlorine disinfection by-products and it may be also detrimental to the filtration processes by membrane fouling (Zazouli et al., 2008). The DOM in lake water is typically classified into two types according to its source. Allochthonous DOM mainly composed of terrestrial humic substances is supplied by runoff from the 
surrounding catchments. Autochthonous DOM is produced from in-lake processes, mainly from carbon excretion of phytoplankton, leachate of dead algal cells and microbial degradation. In general, major components of allochthonous DOM are recalcitrant, humic-like and highly coloured compounds, whereas autochthonous DOM is susceptible to microbial alternations in aquatic environments (McKnight et al., 2001).

Hur et al. (2007) have determined vertical changes of the ratio of protein-like $v s$. humiclike components at four points of the Yongdam reservoir through fluorescence spectroscopy (see Figure 6.13). Bacterial decomposition of the organic detritus is not likely to be very active at low temperature of the bottom water $\left(\sim 5^{\circ} \mathrm{C}\right)$ because metabolic activities of bacteria are positively correlated with water temperature (Wetzel, 2001). In fact, the high content of the protein-like DOM composition in the bottom water conflicts with the vertical distribution of protein amino acids generally described in natural lakes, which depicts a maximum concentration in the epilimnion (Wetzel 2001; $\mathrm{Wu}$ and Tanoue, 2001).
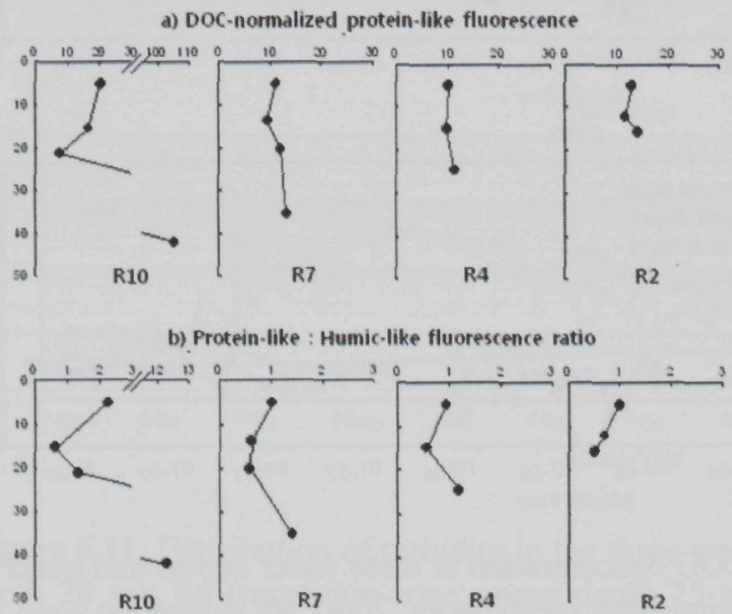

Figure 6.13. (a) Vertical changes of protein-like fluorescence and (b) the ratio of protein-like: humiclike fluorescence on $25^{\text {th }}$ July 2005 .

According to Figure 6.13 (a), a possible explanation for the observation is that although protein-like materials had been originally produced in the upper layers, they are accumulated in the bottom layer due to sinking processes. Because the sinking increases in $\mathrm{R} 10$ due to longer retention time and lower horizontal velocity compared to R7, R4 and R2, the vertical gradient of protein-like fluorescence sharply increases in R10 compared to R7, R4 and R2.

Figure 6.13 (b) shows that the humic-like DOM of the highest concentration in the turbid interflow (middle layer) are mainly transported to the surface layer rather than the bottom layer for the sampling station R4. If the humic-like fluorescences have similar trends with the protein-like fluorescence in the water column, the gradients in Figure 6.11.b would show vertical lines. The highest ratio of protein-like vs. humic-like DOM at R10 shows that the bottom layer in the lacustrine is barely influenced by the turnover of the reservoir water in the late autumn and spring. 


\subsubsection{Eutrophication}

\section{1) Nitrogen}

Values of total nitrogen (TN) were found to be quite high; they amounted to $0.38-5.27$ $\mathrm{mg} / \mathrm{L}$ (mean value: $1.63 \mathrm{mg} / \mathrm{L}$ ) in the surface layer, $0.39-6.00 \mathrm{mg} / \mathrm{L}$ (mean value: 1.50 $\mathrm{mg} / \mathrm{L}$ ) in the middle layer and $0.24-7.44 \mathrm{mg} / \mathrm{L}$ (mean value: $1.52 \mathrm{mg} / \mathrm{L}$ ) in the bottom layer respectively (see Figure 6.14). TN concentrations are higher in the latter stations than in the upper stations of the three layers especially during the summer season in 2005 and 2006.
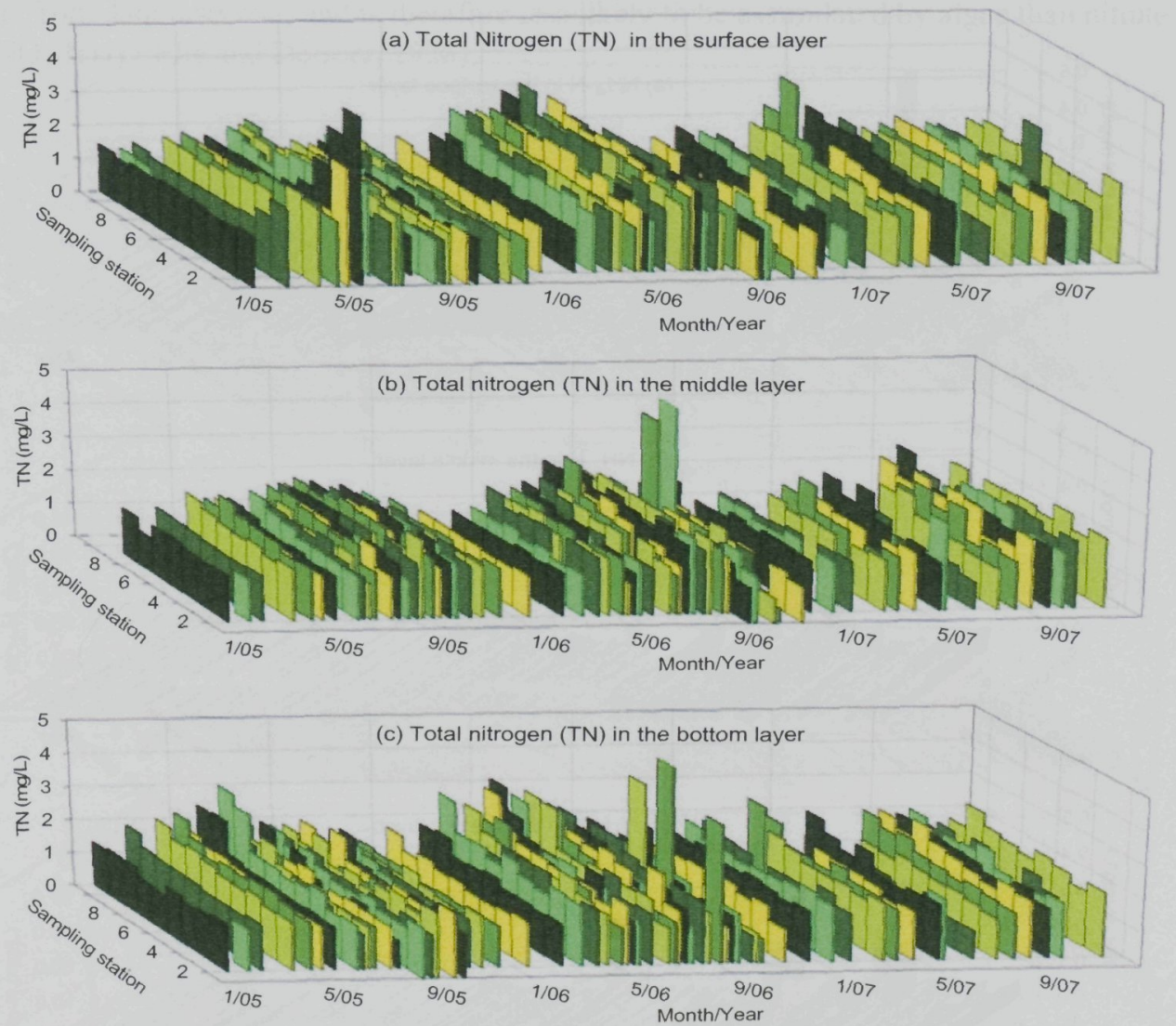

Figure 6.14. Horizontal and vertical distribution of total nitrogen (TN) concentration for the three monitoring years $(2005 \sim 2007)$ at the ten monitoring stations R1-R10.

Figure 6.14 shows that the values of TN are mostly above $0.6 \mathrm{mg} / \mathrm{L}$ in the entire reservoir, which is the upper limit for the raw water quality criteria of drinking water source based on conventional drinking water treatment processes (K MOE, 1987). 
$\mathrm{TN}$ levels are for two reasons important in the reservoir water quality management: in the form of nutrients that can limit growth of phytoplankton (this is generally the case in marine waters) and with respect to health problems. Because nitrogen is not the limiting nutrient for the algal growth in the Yongdam reservoir, the health problems are more important.

Values of ammonia $\left(\mathrm{NH}_{4}-\mathrm{N}\right)$ found were $0.00-1.46 \mathrm{mg} / \mathrm{L}$ (mean value: $0.03 \mathrm{mg} / \mathrm{L}$ ) in the surface layer; $0.01-1.81 \mathrm{mg} / \mathrm{L}$ (mean value: $0.02 \mathrm{mg} / \mathrm{L}$ ) in the middle layer and $0.02-1.53 \mathrm{mg} / \mathrm{L}$ (mean value: $0.03 \mathrm{mg} / \mathrm{L}$ ) in the bottom layer respectively (see Figure 6.15). TN concentrations are higher in the latter stations than in the upper stations of the three layers especially during the summer season in 2005 and 2006.
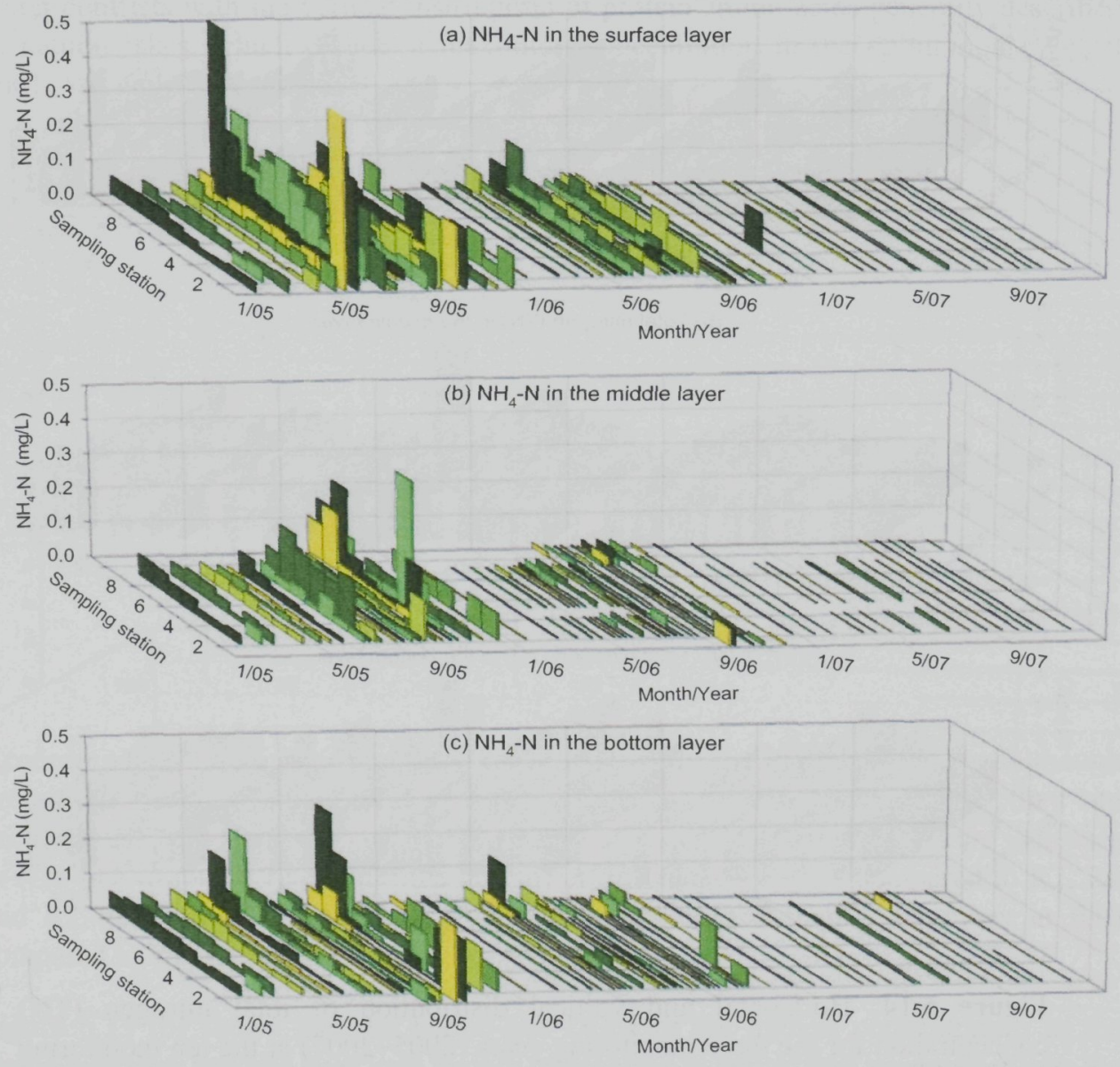

Figure 6.15. Horizontal and vertical distribution of $\mathrm{NH}_{4}-\mathrm{N}$ concentration for the three monitoring years (2005 2007) at the ten monitoring stations R1-R10.

In well-oxygenated reservoir waters, the concentrations of $\mathrm{NH}_{4}-\mathrm{N}$ are generally low, but 
depend on the actual state of metabolism of the aquatic ecosystem. Thus, after a breakdown of phytoplankton populations, higher amounts of $\mathrm{NH}_{4}-\mathrm{N}$ may also be present in the aerated epilimnion before nitrification of the reduced nitrogen compounds. According to Figure 6.15, the higher $\mathrm{NH}_{4}-\mathrm{N}$ in the upper and lower reservoir in all the layers may be results of inflow from the tributaries and of breakdown of algal biomass. In anoxic hypolimnetic waters, $\mathrm{NH}_{4}-\mathrm{N}$ is accumulated due to reduction of nitrate and release of reduced nitrogen compounds from the sediment. Lower values of $\mathrm{NH}_{4}-\mathrm{N}$ in the bottom layer than in the other layers indicate that the bottom layer keeps aerobic conditions.

According to Figure 6.16 , nitrite $\left(\mathrm{NO}_{2}-\mathrm{N}\right)$ was present in much smaller concentrations in the Yongdam reservoir, and is therefore less likely to be assimilated by algae than nitrate $\left(\mathrm{NO}_{3}-\mathrm{N}\right)$ (D'Elia and DeBoer, 1978).
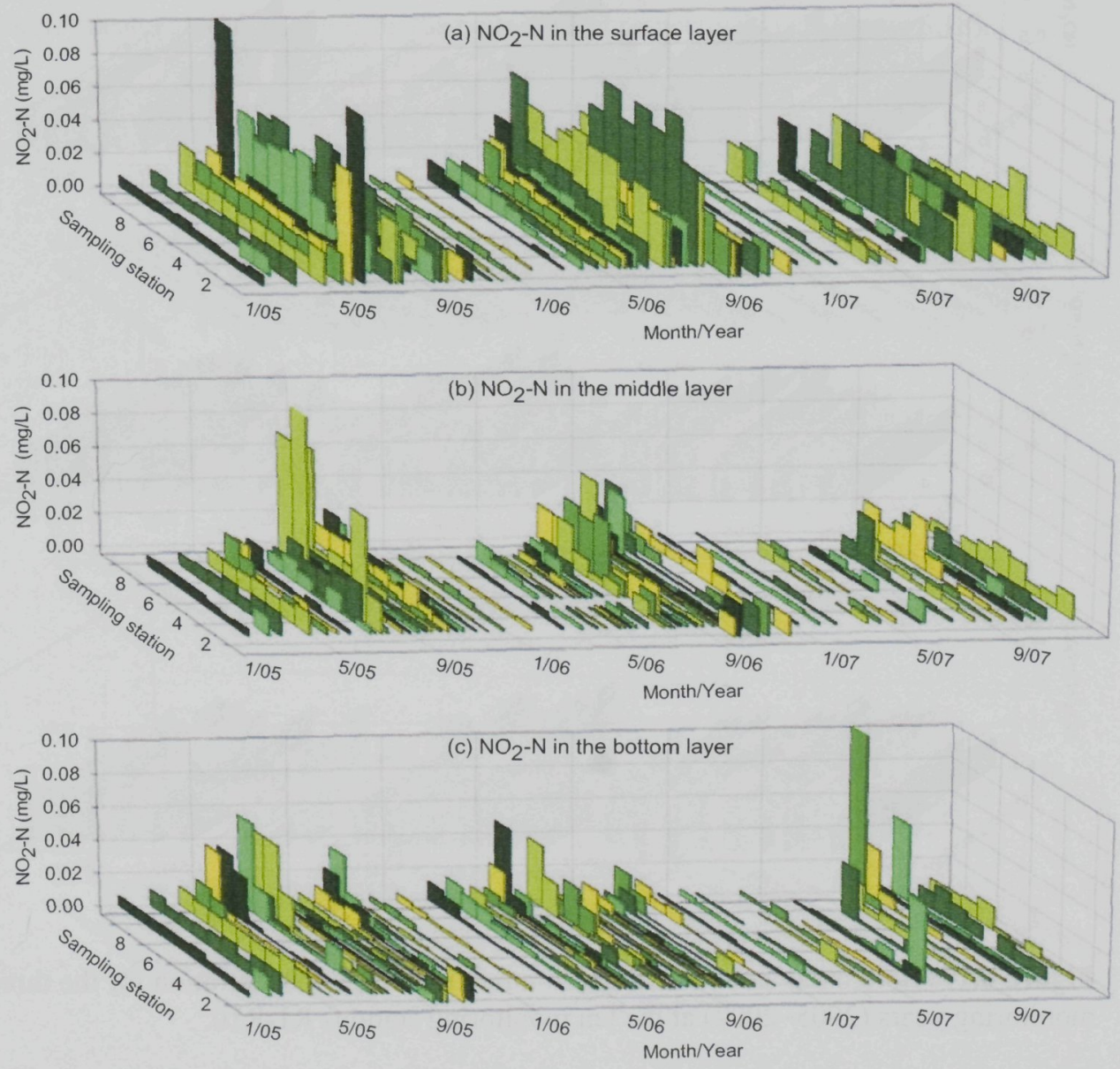

Figure 6.16. Horizontal and vertical distribution of $\mathrm{NO}_{2}-\mathrm{N}$ concentration for the three monitoring years $(2005 \sim 2007)$ at the ten monitoring stations R1-R10. 
Values of $\mathrm{NO}_{2}-\mathrm{N}$ found were $0.00-0.67 \mathrm{mg} / \mathrm{L}$ (mean value: $0.02 \mathrm{mg} / \mathrm{L}$ ) in the surface layer; $0.00-0.13 \mathrm{mg} / \mathrm{L}$ (mean value: $0.01 \mathrm{mg} / \mathrm{L}$ ) in the middle layer and $0.00-0.13$ $\mathrm{mg} / \mathrm{L}$ (mean value: $0.01 \mathrm{~g} / \mathrm{L}$ ) in the bottom layer, respectively (see Figure 6.16 ). According to Figure $6.16, \mathrm{NO}_{2}-\mathrm{N}$ was variable in its distributions over all stations in the surface layer, but showed higher values in latter stations than in the upper stations in the middle and bottom layers.

Values of nitrate $\left(\mathrm{NO}_{3}-\mathrm{N}\right)$ found were $0.00-1.02 \mathrm{mg} / \mathrm{L}$ (mean value: $0.49 \mathrm{mg} / \mathrm{L}$ ) in the surface layer, $0.03-0.92 \mathrm{mg} / \mathrm{L}$ (mean value: $0.50 \mathrm{mg} / \mathrm{L}$ ) in the middle layer and 0.01 $1.20 \mathrm{mg} / \mathrm{L}$ (mean value: $0.48 \mathrm{mg} / \mathrm{L}$ ) in the bottom layer respectively (see Figure 6.17).
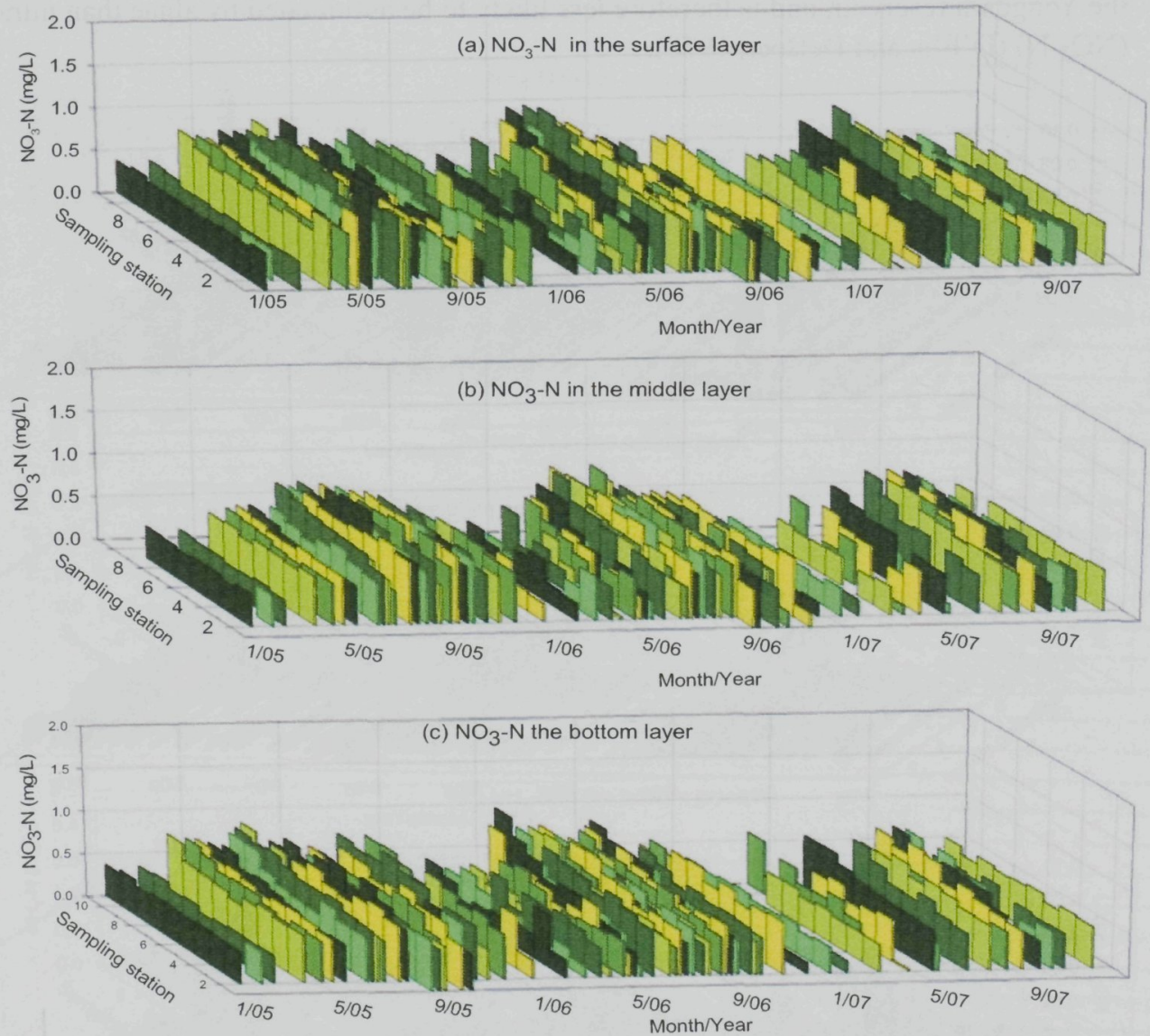

Figure 6.17. Horizontal and vertical distribution of $\mathrm{NO}_{3}-\mathrm{N}$ concentration for the three monitoring years (2005 2007) at the ten monitoring stations R1-R10.

$\mathrm{NO}_{3}-\mathrm{N}$ being the most oxidized form of $\mathrm{N}$ is taken up by algae under aerobic conditions. $\mathrm{TN}$ and $\mathrm{NO}_{3}-\mathrm{N}$ show similar distribution patterns in the entire Yongdam reservoir over the three monitoring years (2005 2007). 
The highest values of $\mathrm{NO}_{3}-\mathrm{N}$ among nitrogen concentrations in the three layers shows that the water column of the Yongdam reservoir always keeps aerobic conditions. Nitrifying bacteria convert $\mathrm{NH}_{4}-\mathrm{N}$ and $\mathrm{NO}_{2}-\mathrm{N}$ to less harmful $\mathrm{NO}_{3}-\mathrm{N}$. However, when a specific nitrate concentration is exceeded (laws in different countries specify $\mathrm{NO}_{3}-\mathrm{N}$ values between $20-50 \mathrm{mg} / \mathrm{L}$ ), there is a danger associated with use of the water for consumption by babies (Straškraba and Tundisi, 1999).

Consequently, since TN concentrations were always below $20 \mathrm{mg} / \mathrm{L}$ over the three monitoring years (2005-2007) at all sampling stations in the Yongdam reservoir, nitrogen levels will not cause these health problems here.

\section{2) Total Phosphorus (TP)}

Values of total phosphorus (TP) found are $0.00-0.17 \mathrm{mg} / \mathrm{L}$ (mean value: $0.02 \mathrm{mg} / \mathrm{L}$ ) in the surface layer, $0.00-0.30 \mathrm{mg} / \mathrm{L}$ (mean value: $0.04 \mathrm{mg} / \mathrm{L}$ ) in the middle layer and $0.00-0.46$ $\mathrm{mg} / \mathrm{L}$ (mean value: $0.04 \mathrm{mg} / \mathrm{L}$ ) in the bottom layer respectively (see Figure 6.18).
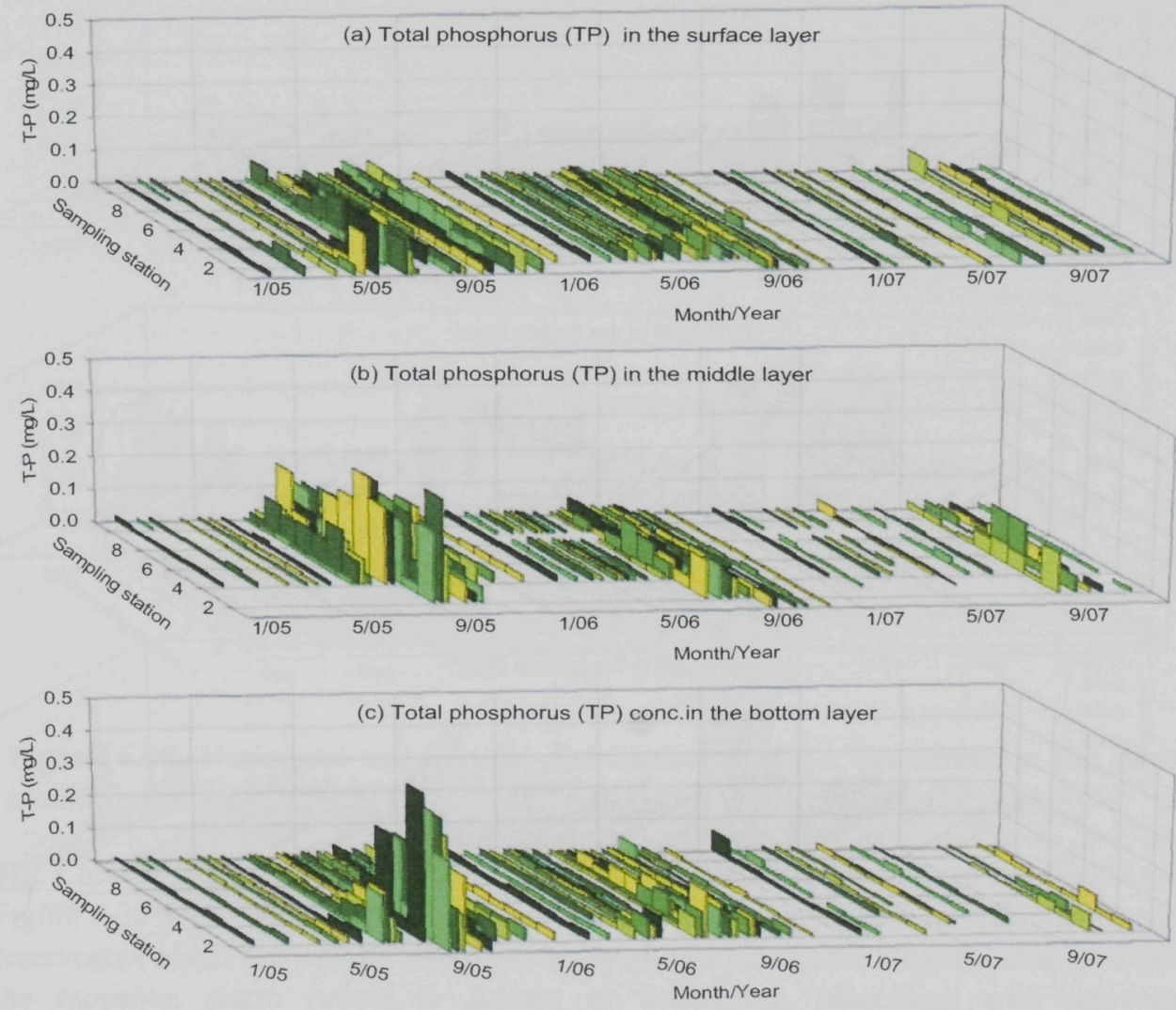

Figure 6.18. Horizontal and vertical distribution of total phosphorus (TP) concentration for the three monitoring years $(2005 \sim 2007)$ at the ten monitoring stations R1-R10. 
The gradient of TP concentration along the reservoir is in opposite direction to other nutrients; it is higher in the upper stations than in the latter stations.

Over the three years 2005-2007, the concentration of TP showed a decreasing trend (see Figure 6.18). The high TP concentrations after the spring and monsoon flood flow in 2005 and 2006 especially in the middle layer of lacustrine zone may be caused by the P loadings from the watershed.

\section{3) Ortho-Phosphorus}

Values of ortho-phosphorus $\left(\mathrm{PO}_{4}-\mathrm{P}\right)$ are $0.00-0.04 \mathrm{mg} / \mathrm{L}$ (mean value: $0.004 \mathrm{mg} / \mathrm{L}$ ) in the surface layer, $0.001-0.094 \mathrm{mg} / \mathrm{L}$ (mean value: $0.01 \mathrm{mg} / \mathrm{L}$ ) in the middle layer and $0.00-0.10 \mathrm{mg} / \mathrm{L}$ (mean value: $0.01 \mathrm{mg} / \mathrm{L}$ ) in the bottom layer respectively (see Figure 6.19). The trend of $\mathrm{PO}_{4}-\mathrm{P}$ concentrations is similar to TP. The average ratio between $\mathrm{PO}_{4}-\mathrm{P}$ and $\mathrm{TP}$ amounted to 0.255 .
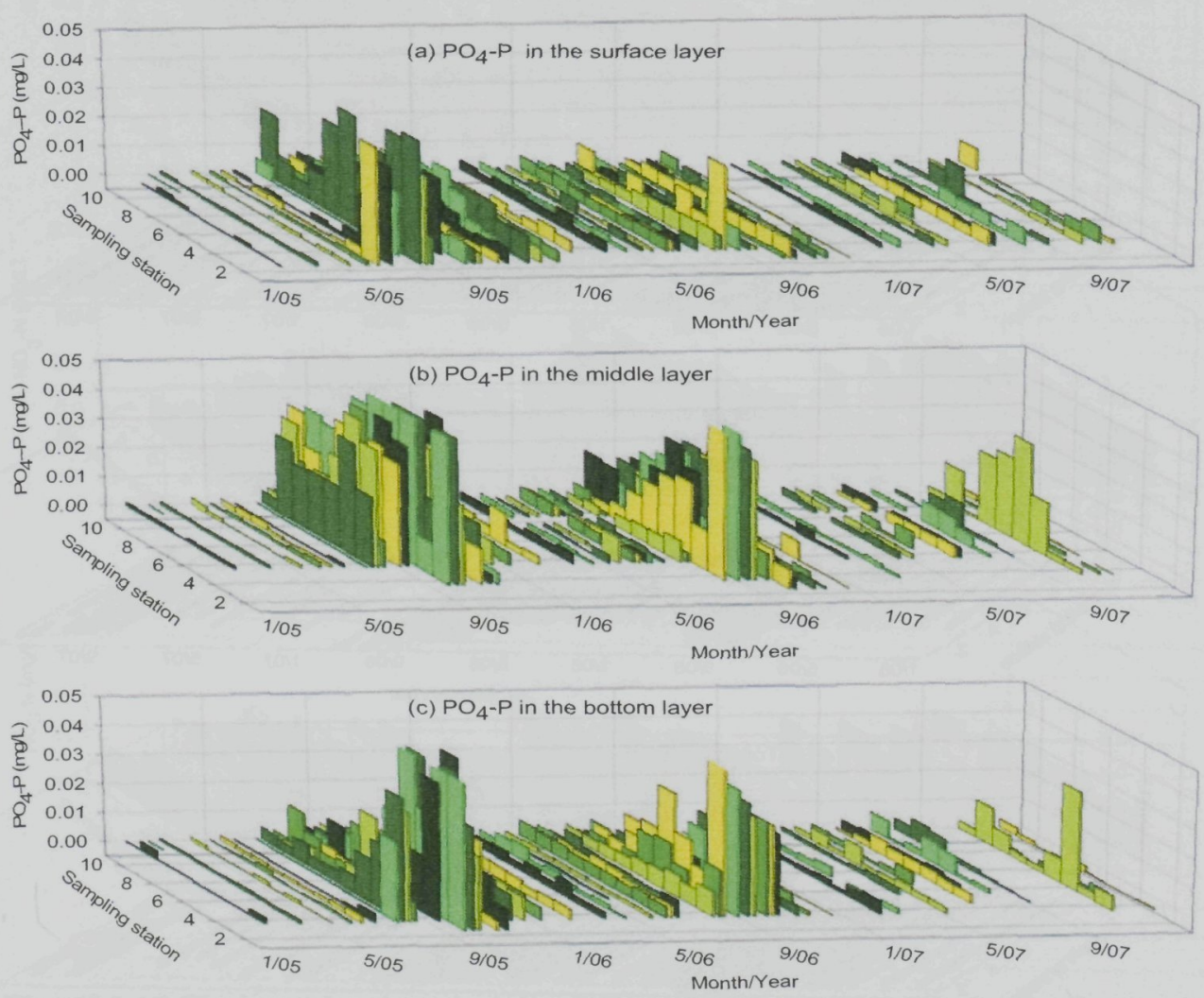

Figure 6.19. Horizontal and vertical distribution of ortho-phosphorus ( $\left.\mathrm{PO}_{4}-\mathrm{P}\right)$ concentration for the three monitoring years (2005 2007) at the ten monitoring stations R1-R10. 


\section{4) Chlorophyll-a}

Values of Chl-a concentration are $1-40 \mu \mathrm{g} / \mathrm{L}$ (mean value: $5 \mu \mathrm{g} / \mathrm{L}$ ) in the surface layer; $1-15 \mu \mathrm{g} / \mathrm{L}$ in the middle layer and $1-13 \mu \mathrm{g} / \mathrm{L}$ in the bottom layer, respectively (see Figure 6.20). The higher levels of Chl-a in the lacustrine zone than in the upper reservoir may be the result of less vertical mixing due to strong thermal stratifications than that in the upper reservoir.
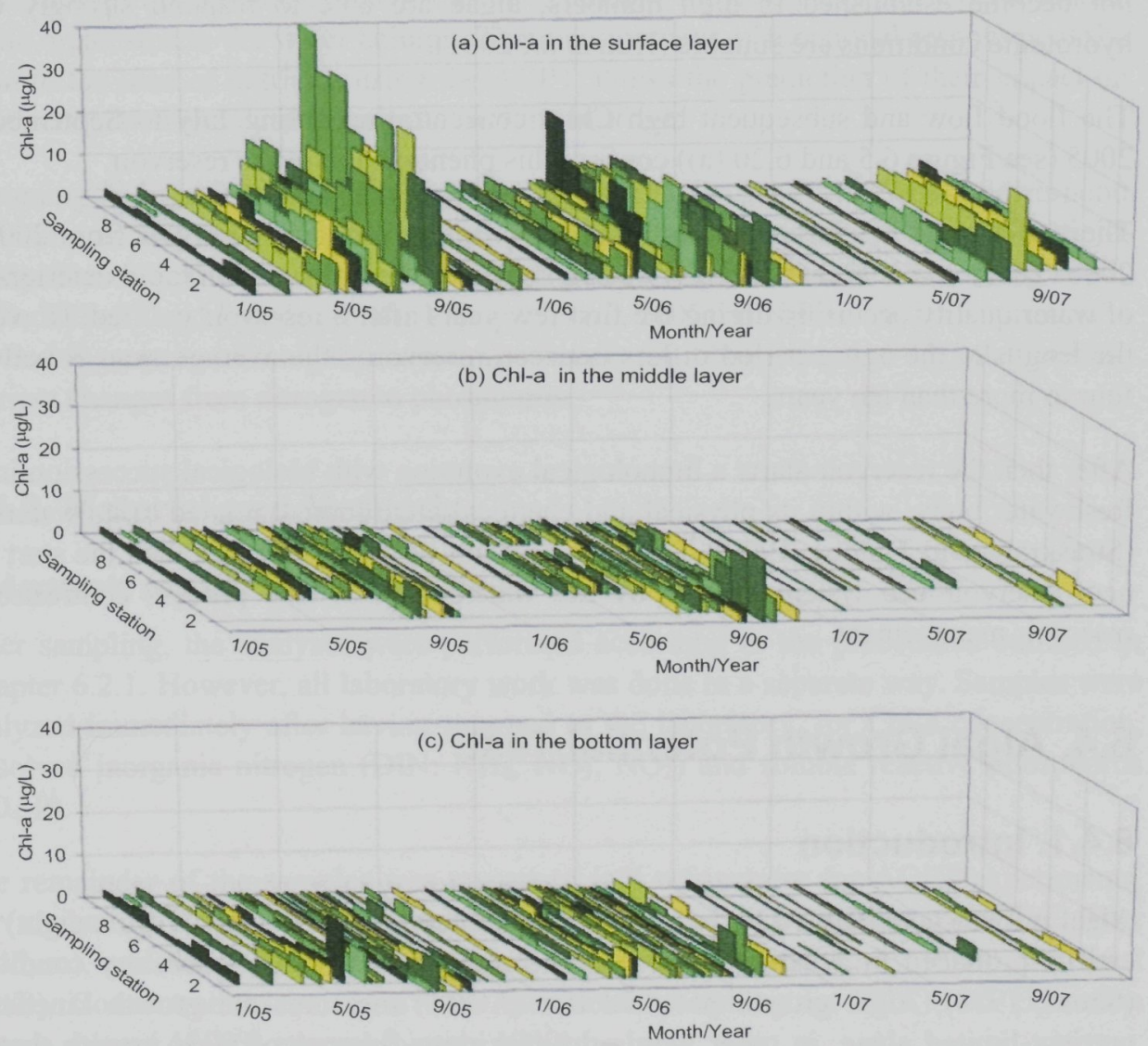

Figure 6.20. Horizontal and vertical distribution of Chl-a concentration the three monitoring years $(2005 \sim 2007)$ at the ten monitoring stations R1-R10.

The Chl-a concentration in the surface layer also shows a decrease over 2005-2007 (see Figure 6.20 (a)). The pattern of seasonal variation of phytoplankton in upstream Korean reservoirs (which are usually deep) generally shows that water blooms occur just after the monsoon storm runoff in August or September, coinciding with increased phosphorus concentration in the surface layer (Kim et al., 2001). The reason of the relatively lower Chl-a concentration in 2007 (see Figure 6.20 (a)) as compared to 2005 and 2006 is probably because of the lack of sunlight due to frequent rainfall. The continuous rise of water level from July to September in 2007 as shown in Figure 6.5, 
suggests that in that year there had been relatively long rainfalls and runoffs as mentioned already in the previous section.

In dry years without heavy monsoon rains, large algal blooms are known to be rare. After the monsoon rains, there are often warm dry periods with intense algal blooms. These correspond to the periods with long residence time that makes it possible for algal biomass to accumulate. Because zooplankton is washed out during the monsoon and has not become established in high numbers, algae are able to respond strongly once hydrologic conditions are suitable (Kim et al, 2001).

The flood flow and subsequent high Chl-a concentration during July to September in 2005 (see Figure 6.5 and 6.20 (a)) confirm this phenomenon in the reservoir.

There is a trend of eutrophication decreasing in the Yongdam reservoir from 2005 to 2006. This trend is in fact different from reservoir aging process with rapid deterioration of water quality occurring during the first few years after a reservoir is filled. However, the length of the aging period differs between reservoirs; the average span is between four to more than ten years.

After then the reservoir starts a limnological evolution with biological succession in the freshwater body, within its physical and chemical environment related to its watershed (Straškraba and Tundisi, 1999). This turning point of the reservoir is then the start of a new reservoir eco-system created by the watershed that is a process controlled by reservoir management.

\subsection{Algal Growth Potential Test}

\subsubsection{Introduction}

Algal growth potential (AGP) is defined as the maximum algal mass (dry weight) that can be produced in a natural water sample under standardized laboratory conditions (Shoaf, 1978). Algal growth potential test (AGPT) measures the growth kinetics of nutrient-limited algae, in other words the maximum amount of algal growth that the nutrients in a water sample can support. This test provides a better indication of the potential for algal growth than can be determined by chemical measurement of nutrient concentrations, because nutrients in the water are not all in a form that can be used by algae, and inhibitors for algal growth exist in the water. (Nyholm and Lyngby, 1988).

The value of AGPT in water samples is that a differentiation can be made between the nutrients of a sample determined by chemical analysis and the nutrients that are actually available for algal growth. The addition of nutrients (usually nitrogen or phosphorus individually or in combination) to the sample can give an indication which nutrient is limiting the algal growth (Miller et al., 1974; Greene et al., 1975; Payne, 1976; Gerhold, 1976; Greene et al., 1976; Shoaf, 1978). 
Since the algal growth potential is defined under standardized laboratory conditions, such potentials may not reflect natural conditions existing in the real environment, such as insufficient light or temperature, grazing by invertebrates or fish, or the presence of toxic materials. Therefore, an understanding of the principles underlying the bioassay test and the factors that affect the algal growth potential is critical for a proper interpretation of the data (Shoaf, 1978); the results from AGPT in the laboratory show larger growth potentials than exists in the real world. Nevertheless, the AGPT is the most straightforward and efficient way to determine the amount of nutrients available to the organisms in the water column. The measurement of functional responses of the biota to the load of nutrients using the AGPT allows the prediction of their impact on algal production and determining the limiting nutrient.

Assuming an algal biomass stoichiometry of $\mathrm{C}_{106} \mathrm{H}_{263} \mathrm{O}_{110} \mathrm{~N}_{16} \mathrm{P}$, this would correspond to an atomic ratio $\mathrm{N}: \mathrm{P}$ of $16: 1$, equivalent with a mass ratio $\mathrm{N}: \mathrm{P}=7.2: 1$ (Redfield, 1958). The critical bio-available N/P ratio is generally taken as 6-10:1 (Forsberg et al., 1978; Nyholm and Lyngby, 1988; Lapointe et al., 2005). If N/P is $<6-10: 1$, nitrogen is the limiting nutrient for algal production, whereas for a N/P value $>6-10: 1$ the limiting nutrient changes from nitrogen to phosphorus.

\subsubsection{Material and Methods}

\section{1) Analysis of DIN, $\mathrm{PO}_{4}-\mathrm{P}$ and $\mathrm{Chl}-\mathrm{a}$}

After sampling, the analyses were performed according to the procedures outlined in Chapter 6.2.1. However, all laboratory work was done in a separate way. Samples were analyzed immediately after having returned to the laboratory, for Chl-a concentration, dissolved inorganic nitrogen (DIN: $\mathrm{NH}_{4}, \mathrm{NO}_{3}, \mathrm{NO}_{2}$ ) and soluble reactive phosphorus $\left(\mathrm{PO}_{4}-\mathrm{P}\right)$.

The remainder of the samples was preserved in a refrigerator for AGPT experiments. For analyzing DIN and $\mathrm{PO}_{4}-\mathrm{P}$, samples were filtered over a Whatman $\mathrm{GF} / \mathrm{F}$ filter $(0.45 \mu \mathrm{m})$, and then analyzed according to the methods described by Rump and Krist (1988). Concentrations of Chl-a were measured by boiling (at $78^{\circ} \mathrm{C}$ ) and extraction using a solvent of $90 \%$ ethanol (Nusch, 1980).

\section{2) Algal Growth Potential Test}

Algal bioassay techniques have been standardized by the National Eutrophication Research Program (NAERP) and the US Environmental Protection Agency (EPA). Two types of bioassay are used, one with the algal species Selenastrum capricornutum, recommended in the EPA protocol, and the other with native algae from each sample site (US EPA, 1971).

In this research, the algal species Microcystis aeruginosa which are native algae in the Yongdam reservoir, were selected for AGPT (APHA, 1995). The test flow is shown in Figure 6.21. 


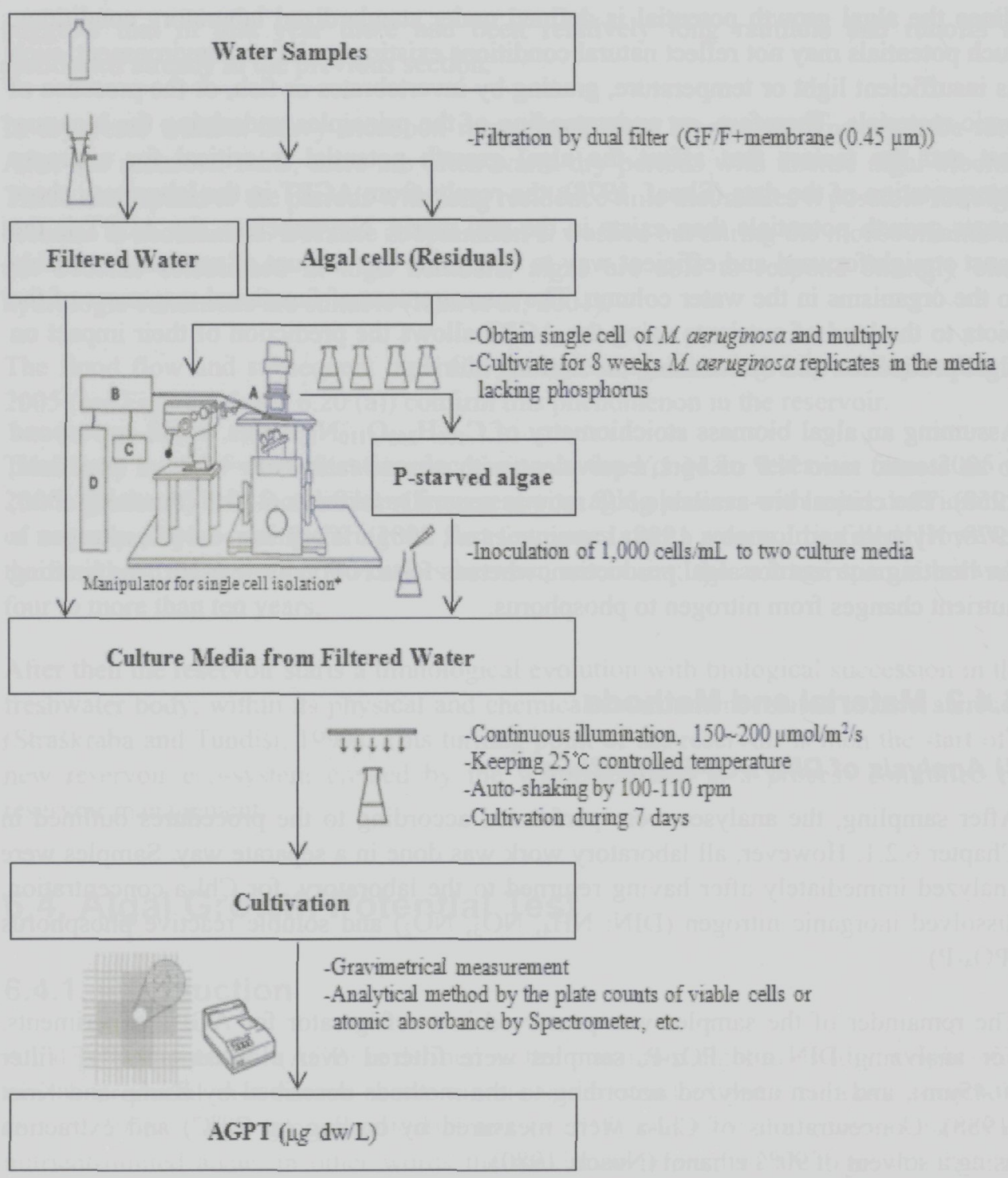

* A: arrangement of microscope, injection pipetteand instrument tube,

B: micromanipulator,

C: joystick, D: gas tank

Figure 6.21. Scheme for algal growth potential test (AGPT)

The water samples were filtered with dual $\mathrm{GF} / \mathrm{F}$ and membrane $(0.45 \mu \mathrm{m})$ filters to separate algal cells and other particulate matter in the water sample. A single algal cell of $M$. aeruginosa was obtained from the filtered algal cells using a manipulator described by Shin (1998) and Beck and Koop (2001), and then the single cell was multiplied in the culture media to ensure a sufficient number of $M$. aeruginosa cells is obtained. Because the cultivated algae have phosphorus in their cells, which can be used 
for producing biomass under phosphorus-limited condition, they were starved for phosphorus before inoculation into the culture media of the sampled water.

The cells of $M$. aeruginosa were fully cultivated under phosphorus starvation for eight weeks by four times duplication in the phosphorus-limited stock culture, MA culture medium (Watababe et al., 1996). The P-starved M. aeruginosa (test algae) of $1.0 \times 10^{3}$ cells $/ \mathrm{mL}$ were injected into the culture media of two filtered water samples of $50 \mathrm{~mL}$ (APHA, 1995; Shin, 1998). The culture media were maintained at $24 \pm 2^{\circ} \mathrm{C}$, and shaken at $100-110 \mathrm{rpm}$ and then cultivated for 7 days under continuous illumination condition of $200 \mu \mathrm{mol} \mathrm{m} \mathrm{s}^{-1}(\mu \mathrm{E})$ to halve the cultivation period.

Finally, for dry weights ( $\mathrm{mg} \mathrm{dw} / \mathrm{L}$ ) of $M$. aeruginosa (test algae), algal cells were harvested by centrifugation and washed twice with distilled water. Then, the pellet was freeze dried. The dry weight of algal biomass was determined gravimetrically.

\subsubsection{Results and Discussion}

\section{1) Limiting Nutrients}

According to Tables 6.4, the Yongdam reservoir can be identified as a phosphoruslimited water body because the N/P ratios were $>10$ in all monitoring stations. However, the minimum N/P ratios in the middle and bottom layers of R1, R2 and R4, and middle layer of R5 were $<10$. The N/P ratios $<10$ in the reservoir were observed in the summer flood season (July-October); however N/P ratios were $>10$ in all tributaries.

The temporal nitrogen limiting conditions in the middle and bottom layers of the upper reservoir in summer implies that there are density water flows including high phosphate concentrations. Because phosphate of non-point sources are loaded by flood flows into the reservoir, and it is transported through the middle and bottom layers due to density differences between river and reservoir water temperature. Also the loaded phosphate can be stored on the bottom of the reservoir. The reason is that phosphate attached or absorbed on surface of eroded soil particle is settled down on the bottom of the upper reservoir section.

\section{2) AGP (Algal growth potential)}

Figure 6.22 and 6.23 show results of $\mathrm{PO}_{4}-\mathrm{P}$ and Algal Growth Potential (AGP) of the Yongdam reservoir in 2005. The variation patterns between $\mathrm{PO}_{4}-\mathrm{P}$ and AGP are very similar. This means that the limiting nutrient of the Yongdam reservoir is phosphorus.

The figures show that the low AGP between autumn turnover of the Yongdam reservoir water and the first flood flow means low load of $\mathrm{PO}_{4}-\mathrm{P}$ from the watershed, because AGP was tested under algal growth conditions, i.e. not natural water temperature (about $\left.6^{\circ} \mathrm{C}\right)$ but normal water temperature $\left(25^{\circ} \mathrm{C}\right)$. 
Table 6.4. DIN, $\mathrm{PO}_{4}-\mathrm{P}, \mathrm{N}: \mathrm{P}$ ratio and $\mathrm{AGP}$ represented by mean $(\mathrm{max} / \mathrm{min})$ in the reservoir in 2005

\begin{tabular}{|c|c|c|c|c|c|c|c|c|c|c|}
\hline \multicolumn{2}{|c|}{ S. Station } & $\begin{array}{l}\text { Level } \\
\text { (EL.m) }\end{array}$ & $\begin{array}{c}\mathrm{DIN} \\
(\mu \mathrm{g} / \mathrm{L})\end{array}$ & $\begin{array}{r}\mathrm{NH}_{4}-\mathrm{N} \\
(\mu \mathrm{g} / \mathrm{L})\end{array}$ & $\begin{array}{r}\mathrm{NO}_{3}-\mathrm{N} \\
(\mu \mathrm{g} / \mathrm{L})\end{array}$ & $\begin{array}{r}\mathrm{NO}_{2}-\mathrm{N} \\
(\mu \mathrm{g} / \mathrm{L})\end{array}$ & $\begin{array}{l}\mathrm{PO}_{4}-\mathrm{P} \\
(\mu \mathrm{g} / \mathrm{L})\end{array}$ & $\begin{array}{c}\text { N/P } \\
\text { Ratio } *^{3}\end{array}$ & $\begin{array}{c}\text { AGP } \\
(m g d w / L)\end{array}$ & $\begin{array}{l}\text { Chl-a } \\
(\mu \mathrm{g} / \mathrm{L})\end{array}$ \\
\hline \multirow{3}{*}{$\begin{array}{c}\mathrm{R} 1 \\
\left(10^{*^{2}}\right)\end{array}$} & $\mathrm{S}^{* 1}$ & $\begin{array}{c}251.9 \\
(259.4 / 241.5)\end{array}$ & $\begin{array}{c}788 \\
(2,194 / 374)\end{array}$ & $\begin{array}{c}118 \\
(547 / 8)\end{array}$ & $\begin{array}{c}613 \\
(1,203 / 262)\end{array}$ & $\begin{array}{c}57 \\
(674 / 0)\end{array}$ & $\begin{array}{c}12 \\
(42 / \mathrm{ND})\end{array}$ & $\begin{array}{c}451.0 \\
(4,684 / 235)\end{array}$ & $\begin{array}{c}3.1 \\
(13 / 0)\end{array}$ & $\begin{array}{c}6 \\
(29 / N D)\end{array}$ \\
\hline & $\mathrm{M}^{* 1}$ & $\begin{array}{c}251.9 \\
(259.4 / 241.5)\end{array}$ & - & - & - & - & - & - & - & - \\
\hline & $\mathrm{B}^{* 1}$ & $\begin{array}{c}251.9 \\
(259.4 / 241.5) \\
\end{array}$ & $\begin{array}{c}609 \\
(749 / 508) \\
\end{array}$ & $\begin{array}{c}97 \\
(220 / 17) \\
\end{array}$ & $\begin{array}{c}501 \\
(588 / 413) \\
\end{array}$ & $\begin{array}{c}11 \\
(16 / 5) \\
\end{array}$ & $\begin{array}{c}34 \\
(99 / 3) \\
\end{array}$ & $\begin{array}{c}76.0 \\
(250 / 6) \\
\end{array}$ & $\begin{array}{c}7 \\
(157 / 0) \\
\end{array}$ & $\begin{array}{c}3 \\
(7 / 1) \\
\end{array}$ \\
\hline \multirow{3}{*}{$\begin{array}{l}\text { R2 } \\
(20)\end{array}$} & $\mathrm{S}$ & $\begin{array}{c}251.9 \\
(259.4 / 241.5)\end{array}$ & $\begin{array}{c}699 \\
(1,636 / 386)\end{array}$ & $\begin{array}{c}96 \\
(704 / 6)\end{array}$ & $\begin{array}{c}575 \\
(824 / 334)\end{array}$ & $\begin{array}{c}27 \\
(231 / \mathrm{ND})\end{array}$ & $\begin{array}{c}9 \\
(40 / \mathrm{ND})\end{array}$ & $\begin{array}{c}397.0 \\
(4,337 / 22)\end{array}$ & $\begin{array}{c}2 \\
(12 / 0)\end{array}$ & $\begin{array}{c}7 \\
(24 / N D)\end{array}$ \\
\hline & M & $\begin{array}{c}251.9 \\
(259.4 / 241.5)\end{array}$ & $\begin{array}{c}616 \\
(711 / 526)\end{array}$ & $\begin{array}{c}635 \\
(157 / 1)\end{array}$ & $\begin{array}{c}534 \\
(625 / 349)\end{array}$ & $\begin{array}{c}19 \\
(62 / 2)\end{array}$ & $\begin{array}{c}36 \\
(94 / 3)\end{array}$ & $\begin{array}{c}70.5 \\
(175 / 7)\end{array}$ & $\begin{array}{c}7 \\
(15 / 0)\end{array}$ & $\begin{array}{c}3 \\
(7 / 1)\end{array}$ \\
\hline & B & $\begin{array}{c}251.9 \\
(259.4 / 241.5)\end{array}$ & $\begin{array}{c}648 \\
(985 / 394)\end{array}$ & $\begin{array}{c}61 \\
(289 / 1) \\
\end{array}$ & $\begin{array}{c}572 \\
(826 / 346)\end{array}$ & $\begin{array}{c}15 \\
(54 / \mathrm{ND})\end{array}$ & $\begin{array}{c}21 \\
(92 / N D)\end{array}$ & $\begin{array}{c}536 \\
(7,664 / 5)\end{array}$ & $\begin{array}{c}5 \\
(15 / 0)\end{array}$ & $\begin{array}{c}3 \\
(14 / \mathrm{ND})\end{array}$ \\
\hline \multirow{3}{*}{$\begin{array}{l}\text { R4 } \\
(25)\end{array}$} & $\mathrm{S}$ & $\begin{array}{c}251.8 \\
(259.4 / 241.5)\end{array}$ & $\begin{array}{c}632 \\
(1,083 / 337)\end{array}$ & $\begin{array}{c}49 \\
(175 / 5)\end{array}$ & $\begin{array}{c}572 \\
(855 / 290)\end{array}$ & $\begin{array}{c}11 \\
(53 / 1)\end{array}$ & $\begin{array}{c}6 \\
(19 / N D)\end{array}$ & $\begin{array}{c}855 \\
(7,742 / 40)\end{array}$ & $\begin{array}{c}2 \\
(6 / 0)\end{array}$ & $\begin{array}{c}5 \\
(17 / \mathrm{ND})\end{array}$ \\
\hline & M & $\begin{array}{c}251.8 \\
(259.4 / 241.5)\end{array}$ & $\begin{array}{c}608 \\
(9383 / 369)\end{array}$ & $\begin{array}{c}33 \\
(80 / 8)\end{array}$ & $\begin{array}{c}565 \\
(848 / 329)\end{array}$ & $\begin{array}{c}11 \\
(79 / 1)\end{array}$ & $\begin{array}{c}21 \\
(86 / N D)\end{array}$ & $\begin{array}{c}470 \\
(6,171 / 8)\end{array}$ & $\begin{array}{c}5 \\
(16 / 0)\end{array}$ & $\begin{array}{c}2 \\
(4 / N D)\end{array}$ \\
\hline & B & $\begin{array}{c}251.8 \\
(259.4 / 241.5) \\
\end{array}$ & $\begin{array}{c}589 \\
(829 / 328) \\
\end{array}$ & $\begin{array}{c}25 \\
(64 / 6) \\
\end{array}$ & $\begin{array}{c}559 \\
(796 / 308) \\
\end{array}$ & $\begin{array}{c}5 \\
(20 / 1) \\
\end{array}$ & $\begin{array}{c}18 \\
(64 / N D)\end{array}$ & $\begin{array}{c}889 \\
(5,698 / 7)\end{array}$ & $\begin{array}{c}5 \\
(16 / 0) \\
\end{array}$ & $\begin{array}{c}2 \\
(4 / \mathrm{ND}) \\
\end{array}$ \\
\hline \multirow{3}{*}{$\begin{array}{l}\text { R5 } \\
(35)\end{array}$} & $\mathrm{S}$ & $\begin{array}{c}251.9 \\
(259.4 / 241.5)\end{array}$ & $\begin{array}{c}614 \\
(1,080 / 359)\end{array}$ & $\begin{array}{c}51 \\
(191 / 6)\end{array}$ & $\begin{array}{c}553 \\
(871 / 265)\end{array}$ & $\begin{array}{c}10 \\
(40 / 1)\end{array}$ & $\begin{array}{c}7 \\
(38 / N D)\end{array}$ & $\begin{array}{c}820 \\
(8,308 / 28)\end{array}$ & $\begin{array}{c}2 \\
(12 / 0)\end{array}$ & $\begin{array}{c}6 \\
(30 / \mathrm{ND})\end{array}$ \\
\hline & M & $\begin{array}{c}251.9 \\
(259.4 / 241.5)\end{array}$ & $\begin{array}{c}630 \\
(984 / 292)\end{array}$ & $\begin{array}{c}29 \\
(170 / 4)\end{array}$ & $\begin{array}{c}590 \\
(883 / 285)\end{array}$ & $\begin{array}{c}11 \\
(102 / 1)\end{array}$ & $\begin{array}{c}26 \\
(80 / \mathrm{ND})\end{array}$ & $\begin{array}{c}902 \\
(6,120 / 7)\end{array}$ & $\begin{array}{c}7 \\
(16 / 0)\end{array}$ & $\begin{array}{c}2 \\
(4 / \mathrm{ND})\end{array}$ \\
\hline & B & $\begin{array}{c}251.9 \\
(259.4 / 241.5) \\
\end{array}$ & $\begin{array}{c}570 \\
(835 / 350) \\
\end{array}$ & $\begin{array}{c}19 \\
(99 / 3)\end{array}$ & $\begin{array}{c}548 \\
(814 / 331) \\
\end{array}$ & $\begin{array}{c}3 \\
(10 / N D)\end{array}$ & $\begin{array}{c}9 \\
(27 / N D)\end{array}$ & $\begin{array}{c}1,278 \\
(5,847 / 18)\end{array}$ & $\begin{array}{c}2 \\
(8 / 0) \\
\end{array}$ & $\begin{array}{c}1 \\
(2 / \mathrm{ND})\end{array}$ \\
\hline \multirow{3}{*}{$\begin{array}{c}\text { R6 } \\
(40)\end{array}$} & $\mathrm{S}$ & $\begin{array}{c}251.9 \\
(259.4 / 241.5)\end{array}$ & $\begin{array}{c}595 \\
(1,052 / 334)\end{array}$ & $\begin{array}{c}45 \\
(151 / 5)\end{array}$ & $\begin{array}{c}540 \\
(866 / 279)\end{array}$ & $\begin{array}{c}10 \\
(45 / 1)\end{array}$ & $\begin{array}{c}6 \\
(31 / N D)\end{array}$ & $\begin{array}{c}1,285 \\
(7,814 / 34)\end{array}$ & $\begin{array}{c}1 \\
(10 / 0)\end{array}$ & $\begin{array}{c}5 \\
(20 / N D)\end{array}$ \\
\hline & M & $\begin{array}{c}251.9 \\
(259.4 / 241.5)\end{array}$ & $\begin{array}{c}614 \\
(954 / 359)\end{array}$ & $\begin{array}{c}26 \\
(126 / 5)\end{array}$ & $\begin{array}{c}583 \\
(944 / 320)\end{array}$ & $\begin{array}{c}5 \\
(15 / \mathrm{ND})\end{array}$ & $\begin{array}{c}18 \\
(54 / \mathrm{ND})\end{array}$ & $\begin{array}{c}862 \\
(5,269 / 13)\end{array}$ & $\begin{array}{c}5 \\
(17 / 0)\end{array}$ & $\begin{array}{c}1 \\
(3 / \mathrm{ND})\end{array}$ \\
\hline & B & $\begin{array}{c}251.9 \\
(259.4 / 241.5) \\
\end{array}$ & $\begin{array}{c}554 \\
(850 / 318) \\
\end{array}$ & $\begin{array}{c}16 \\
(81 / 1)\end{array}$ & $\begin{array}{c}536 \\
(845 / 265)\end{array}$ & $\begin{array}{c}2 \\
(10 / \mathrm{ND})\end{array}$ & $\begin{array}{c}3 \\
(10 / \mathrm{ND})\end{array}$ & $\begin{array}{c}929 \\
(5,422 / 70)\end{array}$ & $\begin{array}{c}0.1 \\
(2 / 0)\end{array}$ & $\begin{array}{c}1 \\
(2 / \mathrm{ND})\end{array}$ \\
\hline \multirow{3}{*}{$\begin{array}{l}\text { R7 } \\
(36)\end{array}$} & $\mathrm{S}$ & $\begin{array}{c}251.9 \\
(259.4 / 241.5)\end{array}$ & $\begin{array}{c}600 \\
(1,022 / 356)\end{array}$ & $\begin{array}{c}42 \\
(160 / 6)\end{array}$ & $\begin{array}{c}548 \\
(877 / 294)\end{array}$ & $\begin{array}{c}10 \\
(59 / 1)\end{array}$ & $\begin{array}{c}4.5 \\
(11 / \mathrm{ND})\end{array}$ & $\begin{array}{c}1,258 \\
(7,779 / 56)\end{array}$ & $\begin{array}{c}1 \\
(3 / 0)\end{array}$ & $\begin{array}{c}5 \\
(22 / \mathrm{ND})\end{array}$ \\
\hline & $\mathrm{M}$ & $\begin{array}{c}251.9 \\
(259.4 / 241.5)\end{array}$ & $\begin{array}{c}658 \\
(1,142 / 331)\end{array}$ & $\begin{array}{c}39 \\
(377 / 6)\end{array}$ & $\begin{array}{c}614 \\
(959 / 287)\end{array}$ & $\begin{array}{c}5 \\
(16 / 1)\end{array}$ & $\begin{array}{c}15 \\
(46 / N D)\end{array}$ & $\begin{array}{c}1,049 \\
(5,922 / 16)\end{array}$ & $\begin{array}{c}5 \\
(15 / 0)\end{array}$ & $\begin{array}{c}1 \\
(3 / \mathrm{ND})\end{array}$ \\
\hline & B & $\begin{array}{c}251.9 \\
(259.4 / 241.5) \\
\end{array}$ & $\begin{array}{c}567 \\
(805 / 343) \\
\end{array}$ & $\begin{array}{c}16 \\
(71 / \mathrm{ND}) \\
\end{array}$ & $\begin{array}{c}549 \\
(800 / 295) \\
\end{array}$ & $\begin{array}{c}2.2 \\
(11 / 1) \\
\end{array}$ & $\begin{array}{c}3 \\
(8 / \mathrm{ND}) \\
\end{array}$ & $\begin{array}{c}1,151 \\
(5,700 / 74) \\
\end{array}$ & $\begin{array}{c}0.1 \\
(2 / 0) \\
\end{array}$ & $\begin{array}{c}1 \\
(1 / \mathrm{ND})\end{array}$ \\
\hline \multirow{3}{*}{$\begin{array}{l}\text { R10 } \\
(43)\end{array}$} & $\mathrm{S}$ & $\begin{array}{c}251.9 \\
(259.4 / 241.5)\end{array}$ & $\begin{array}{c}577 \\
(1,026 / 358)\end{array}$ & $\begin{array}{c}44 \\
(202 / 6)\end{array}$ & $\begin{array}{c}523 \\
(774 / 313)\end{array}$ & $\begin{array}{c}10 \\
(50 / 2)\end{array}$ & $\begin{array}{c}5 \\
(22 / N D)\end{array}$ & $\begin{array}{c}585 \\
(4,428 / 47)\end{array}$ & $\begin{array}{c}1 \\
(7 / 0)\end{array}$ & $\begin{array}{c}6 \\
(40 / N D)\end{array}$ \\
\hline & M & $\begin{array}{c}251.9 \\
(259.4 / 241.5)\end{array}$ & $\begin{array}{c}580 \\
(785 / 345)\end{array}$ & $\begin{array}{c}22 \\
(116 / 3)\end{array}$ & $\begin{array}{c}554 \\
(777 / 302)\end{array}$ & $\begin{array}{c}4 \\
(18 / \mathrm{ND})\end{array}$ & $\begin{array}{c}9 \\
(34 / \mathrm{ND})\end{array}$ & $\begin{array}{c}797 \\
(5,044 / 19)\end{array}$ & $\begin{array}{c}2 \\
(11 / 0)\end{array}$ & $\begin{array}{c}1 \\
(2 / \mathrm{ND})\end{array}$ \\
\hline & B & $\begin{array}{c}251.9 \\
(259.4 / 241.5)\end{array}$ & $\begin{array}{c}524 \\
(673 / 369)\end{array}$ & $\begin{array}{c}26 \\
(159 / \mathrm{ND})\end{array}$ & $\begin{array}{c}495 \\
(662 / 322)\end{array}$ & $\begin{array}{c}2 \\
(13 / N D)\end{array}$ & $\begin{array}{c}2 \\
(6 / \mathrm{ND})\end{array}$ & $\begin{array}{c}1,696 \\
(6,644 / 77)\end{array}$ & $\begin{array}{c}0.1 \\
(1 / 0)\end{array}$ & $\begin{array}{c}1 \\
(2 / N D)\end{array}$ \\
\hline
\end{tabular}

${ }^{* 1} \mathrm{~S}$ stands for the surface layer; $\mathrm{M}$ for the middle layer; B for the bottom layer. The middle layer of the R1 station was not sampled because of its shallow water depth.

$*^{2}$ Annual mean depth (m)

${ }^{*} \mathrm{~N} / \mathrm{P}$ stands for the average of the N/P ratio in each measurement. Therefore it is different from the ratio between DIN and $\mathrm{PO}_{4}-\mathrm{P}$ in the Table.

ND: not detectable 

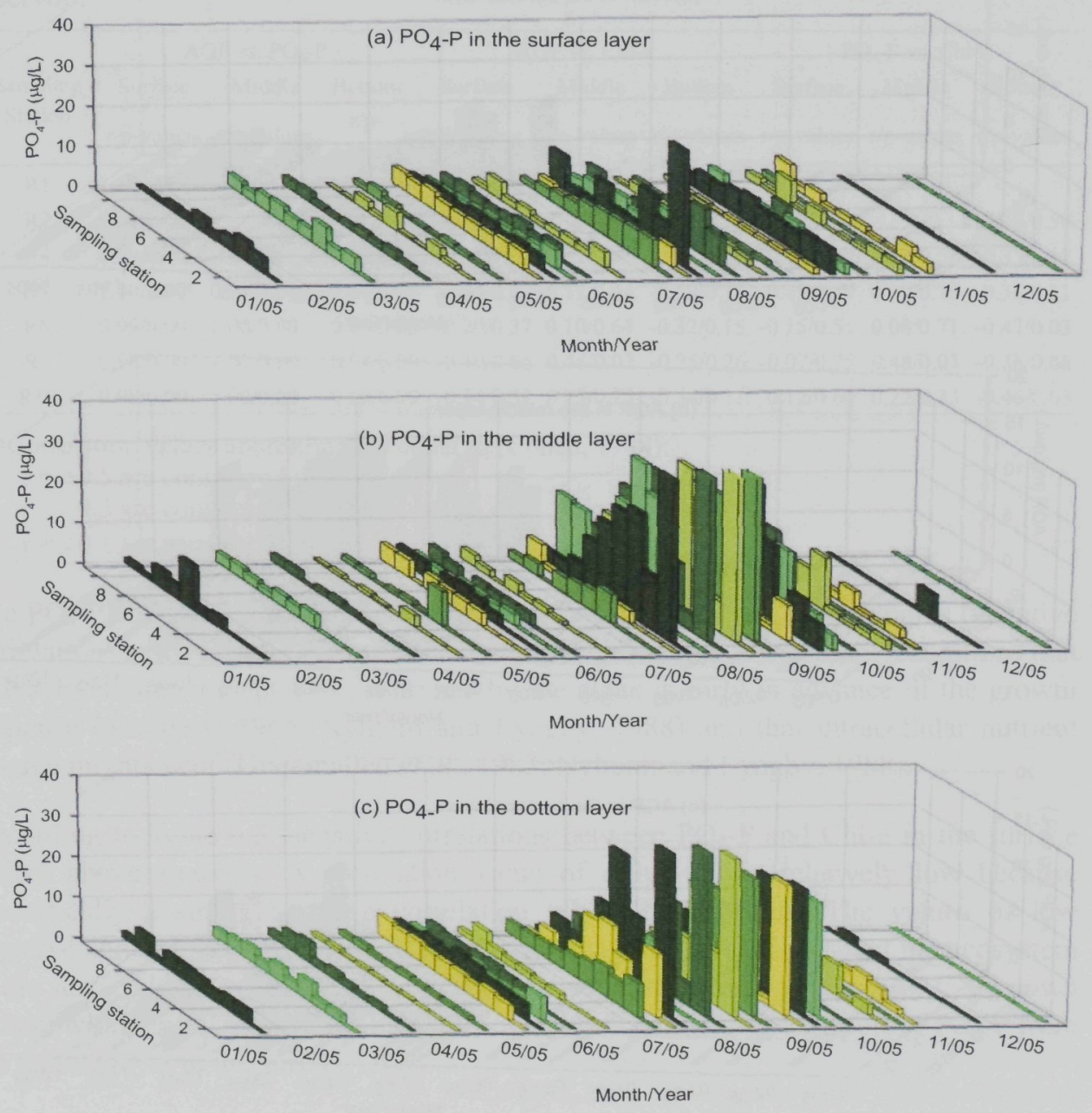

Figure 6.22. Spatial and temporal variations of $\mathrm{PO}_{4}-\mathrm{P}$ concentration in the Yongdam reservoir in 2005 .

The AGPT assumes a good relationship between a limiting nutrient and its contribution to algal growth; therefore a correlation analysis between limiting nutrient (here $\mathrm{PO}_{4}-\mathrm{P}$ ) and AGP identifies the effectiveness of AGPT as an indication of the potential for algal growth.

According to the correlation analysis, algal growth potential (AGP) closely correlates with $\mathrm{PO}_{4}-\mathrm{P}$ in the entire reservoir, with strong positive correlation coefficients $0.9 \sim 1.0$ and confidence levels $100 \%$ (1-p value) (see Table 6.5). 

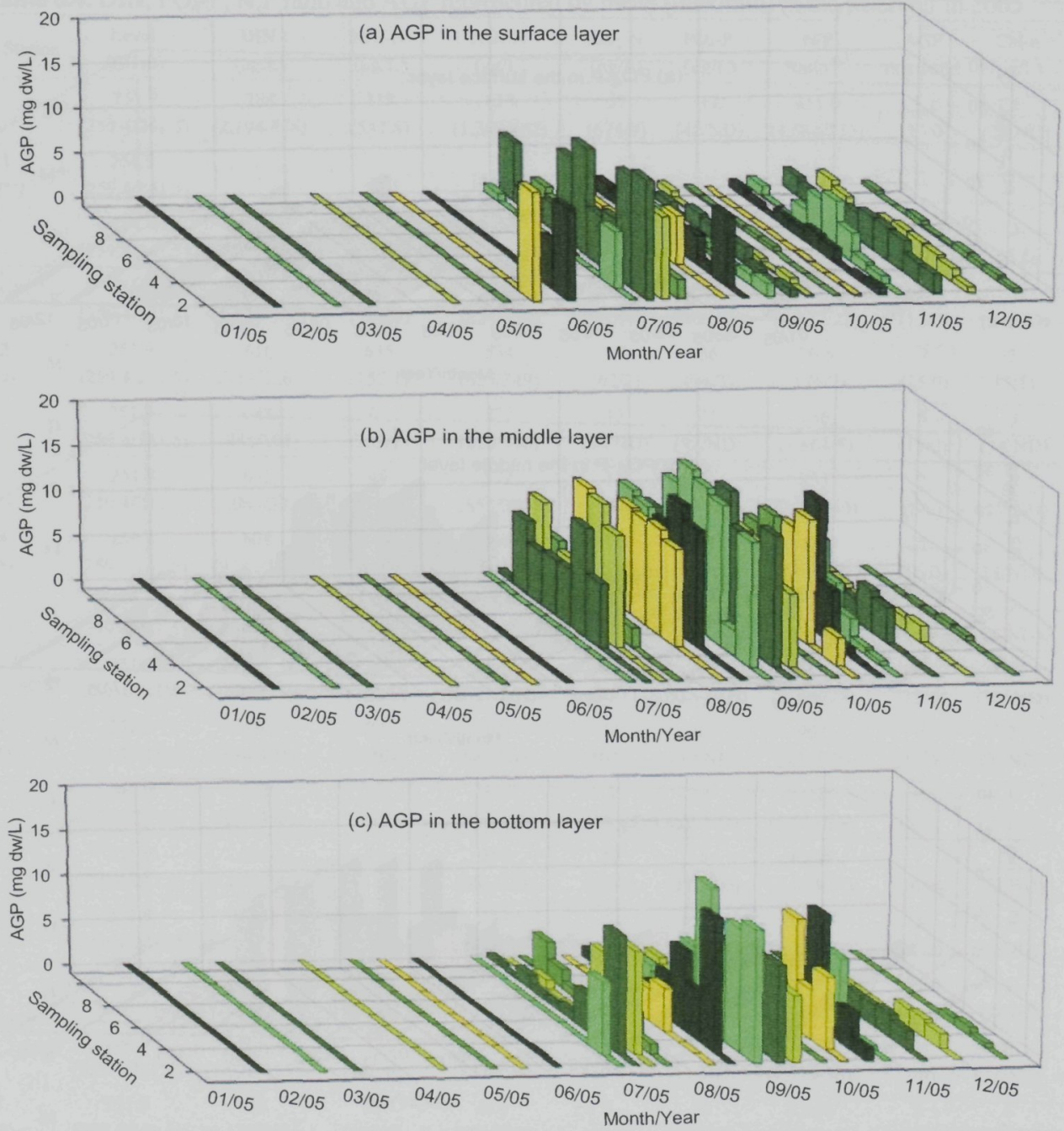

Figure 6.23. Spatial and temporal variations of AGPT in the Yongdam reservoir in 2005.

In the surface and the bottom layers, negative correlations between $\mathrm{PO}_{4}-\mathrm{P}$ and $\mathrm{Chl}-\mathrm{a}$ indicate that the $\mathrm{PO}_{4}-\mathrm{P}$ and $\mathrm{Chl}-\mathrm{a}$ values vary inversely; however, the significance levels are larger than 0.05 ( $95 \%$ in confidence level).

In the middle layers, although there are positive correlations between AGP (or $\mathrm{PO}_{4}-\mathrm{P}$ ) and Chl-a show positive, the most $\mathrm{p}$-values are larger the significance level 0.05 . Only a few sampling stations in the middle and bottom layers, where there are not photosynthetic activity, show p-values that are smaller than the significance level 0.05 . 
Table 6.5. Correlation analysis between $\mathrm{AGP}, \mathrm{PO}_{4}-\mathrm{P}$ and $\mathrm{Chl}-\mathrm{a}$ at main stream of the reservoir.

\begin{tabular}{|c|c|c|c|c|c|c|c|c|c|}
\hline \multirow[b]{2}{*}{$\begin{array}{l}\text { Sampling } \\
\text { Station }\end{array}$} & \multicolumn{3}{|c|}{ AGP vs. $\mathrm{PO}_{4}-\mathrm{P}$} & \multicolumn{3}{|c|}{ AGP vs. Chl-a } & \multicolumn{3}{|c|}{$\mathrm{PO}_{4}-\mathrm{P}$ vs. Chl-a } \\
\hline & $\begin{array}{l}\text { Surface } \\
\text { r/p-values }\end{array}$ & $\begin{array}{c}\text { Middle } \\
\text { r/p-values }\end{array}$ & $\begin{array}{c}\text { Bottom } \\
\mathrm{r} / \mathrm{p}- \\
\text { values }\end{array}$ & $\begin{array}{l}\text { Surface } \\
\text { r/p-values }\end{array}$ & $\begin{array}{c}\text { Middle } \\
\text { r/p-values }\end{array}$ & $\begin{array}{l}\text { Bottom } \\
\text { r/p-values }\end{array}$ & $\begin{array}{l}\text { Surface } \\
\text { r/p-values }\end{array}$ & $\begin{array}{c}\text { Middle } \\
\text { r/p-values }\end{array}$ & $\begin{array}{c}\text { Bottom } \\
\text { r/p-values }\end{array}$ \\
\hline R1 & $1.00 / 0.00$ & & & $0.00 / 1.00$ & & & $0.01 / 0.97$ & & \\
\hline R2 & $1.00 / 0.00$ & & $0.86 / 0.00$ & $-0.18 / 0.44$ & & $-0.21 / 0.38$ & $-0.17 / 0.48$ & & $-0.13 / 0.59$ \\
\hline R4 & $0.99 / 0.00$ & $0.94 / 0.00$ & $0.94 / 0.00$ & $-0.20 / 0.38$ & $0.11 / 0.63$ & $-0.38 / 0.09$ & $-0.15 / 0.52$ & $0.13 / 0.58$ & $-0.35 / 0.12$ \\
\hline R5 & $1.00 / 0.00$ & $0.95 / 0.00$ & 1.00 .0 .00 & $-0.22 / 0.32$ & $0.17 / 0.44$ & $-0.32 / 0.15$ & $-0.19 / 0.39$ & $0.09 / 0.70$ & $-0.35 / 0.11$ \\
\hline R6 & $0.99 / 0.00$ & $1.00 / 0.00$ & $0.95 / 0.00$ & $-0.20 / 0.37$ & $0.10 / 0.64$ & $-0.32 / 0.15$ & $-0.15 / 0.51$ & $0.08 / 0.71$ & $-0.47 / 0.03$ \\
\hline R7 & $0.98 / 0.00$ & $1.00 / 0.00$ & $0.94 / 0.00$ & $-0.10 / 0.65$ & $0.48 / 0.02$ & $-0.25 / 0.26$ & $-0.07 / 0.75$ & $0.48 / 0.03$ & $-0.38 / 0.08$ \\
\hline $\mathrm{R} 10$ & $0.98 / 0.00$ & $1.00 / 0.00$ & $0.90 / 0.00$ & $-0.14 / 0.52$ & $0.25 / 0.25$ & $-0.34 / 0.11$ & $-0.12 / 0.60$ & $0.22 / 0.33$ & $-0.46 / 0.03$ \\
\hline
\end{tabular}

The $\mathrm{PO}_{4}-\mathrm{P}$ concentration must be low prior to the appearance of algal blooms (negative correlation) in the aquatic system. The reason for this is that it was early recognized that in batch cultures the uptake of nutrients by the algae is early in advance of the growth response (Skulberg, 1966; Nyholm and Lyngby, 1988) and that intracellular nutrient storage might occur (Giammatteo et al., 1983; Nyholm and Lyngby, 1988).

According to Table 6.5, negative correlations between $\mathrm{PO}_{4}-\mathrm{P}$ and $\mathrm{Chl}-\mathrm{a}$ in the surface reflect above findings. A correlation value of only -0.2 is relatively low because theoretically a strong negative correlation would be expected. The values of low negative and non correlation, $-0.2<\mathrm{r}<0$, in the surface layer means that the ecosystem in the surface layer behaves in a very complex way. There can be two reasons: unknown factors with respect to the algal metabolism and/or a continuous transport of $\mathrm{PO}_{4}-\mathrm{P}$ from the middle layer to the surface layer.

The distribution of positive correlation coefficients between $\mathrm{PO}_{4}-\mathrm{P}$ and $\mathrm{Chl}-\mathrm{a}$ in the middle layers indicates that there are the higher concentration of $\mathrm{PO}_{4}-\mathrm{P}$ in the middle layers than in the surface and bottom layers, with the algal particles being settled down from the surface layer, regardless of whether or not algal growth in the middle layers takes place during the summer period.

In the bottom layer, the values of low negative correlation, $-0.5<\mathrm{r}<-0.1$ between $\mathrm{PO}_{4}$ $\mathrm{P}$ and Chl-a identify that there is a steep decrease of algal cells without algal growth, but the $\mathrm{PO}_{4}-\mathrm{P}$ is slowly accumulated.

Therefore these stronger correlations in the middle and bottom layers than in the surface layer identify that only a physical such as mixing behavior and sunlight affect algal growth with more close correlations than nutrient. Consequently it can be concluded 
that correlation between AGP (or $\mathrm{PO}_{4}-\mathrm{P}$ ) and $\mathrm{Chl}-\mathrm{a}$ in the Yongdam reservoir shows mostly weak, and has a low confidence level.

If there are no other factors hindering algal growth, the possible total Chl-a concentration may be the sum of a measured Chl-a concentration and AGP. However, one serious weakness in the use of Chl-a is the great variability of cellular chlorophyll content. This may vary between $0.25 \%$ and $10 \%$ of the fresh algal weight, depending on the algal species (Bowie et al., 1985). Great variability in individual cases can be expected, either on a seasonal or annual basis, due to species composition, light conditions, nutrient availability, and so on. The ratio between algal biomass and Chl-a is often taken as 145 for water quality modelling (Cole and Wells, 2003). But, for example, according to Lee et al. (2000), the mean ratio between the biomass and Chl-a of $M$. aeruginosa cultivated under various $\mathrm{N}$ - and P-fixed conditions, amounts to 292.

When using these ratios of 145 and 292, the potential Chl-a concentrations for the lacustrine zone (R10) in the surface, middle and bottom layers take an average value of $3-6,8-15$ and $1 \mu \mathrm{g} / \mathrm{L}$, and a maximum of $22-45,36-100$ and $4-8 \mu \mathrm{g} / \mathrm{L}$ respectively. These concentrations are quite high even though they have been deduced under laboratory conditions.

\section{3) AGP Distribution in the Thermally Stratified Reservoir}

The results of AGP along the main stream of the Yongdam reservoir, except for its tributaries, are shown in Figure.6.24, which has been redrawn from Figure 6.22 and 6.23 to identify the gradients of nutrient variations. Since the Yongdam reservoir is very long (about $38.5 \mathrm{~km}$ ) compared to its width (about $1.2 \mathrm{~km}$ ) and has a dendritic shape, there are large longitudinal variations in the water quality.

According to Figure 6.24 (a), (b) and (c), variations of the AGP, $\mathrm{PO}_{4}-\mathrm{P}$ and Chl-a concentrations between the sampling stations and layers suggest that there are different biological and physical mixing processes in the Yongdam reservoir, i.e. in the riverine, transitional and lacustrine zones. These are mainly caused by changes in the hydraulic conditions due to the reservoir geomorphology and thermal stratification (Kimmel and Groeger, 1984).

According to Figure 6.24, station R2 shows variations of Chl-a in the surface and bottom layers, but the middle layer is not well developed due to the shallow water depth at R2. This implies that these zones should be characterized as riverine.

The $\mathrm{PO}_{4}-\mathrm{P}$ and AGP values in both the middle and bottom layers of R4 and R5 are high, but show decreasing trend, and $\mathrm{Chl}-\mathrm{a}$ is disappearing. In station $\mathrm{R} 6, \mathrm{PO}_{4}-\mathrm{P}$ and AGP are also disappearing in the bottom layer. This implies that the flow conditions at stations R4 and R5 are changing due to the reservoir geomorphological conditions. Therefore R4 and R5 can be considered as the reservoir's transition zone. 


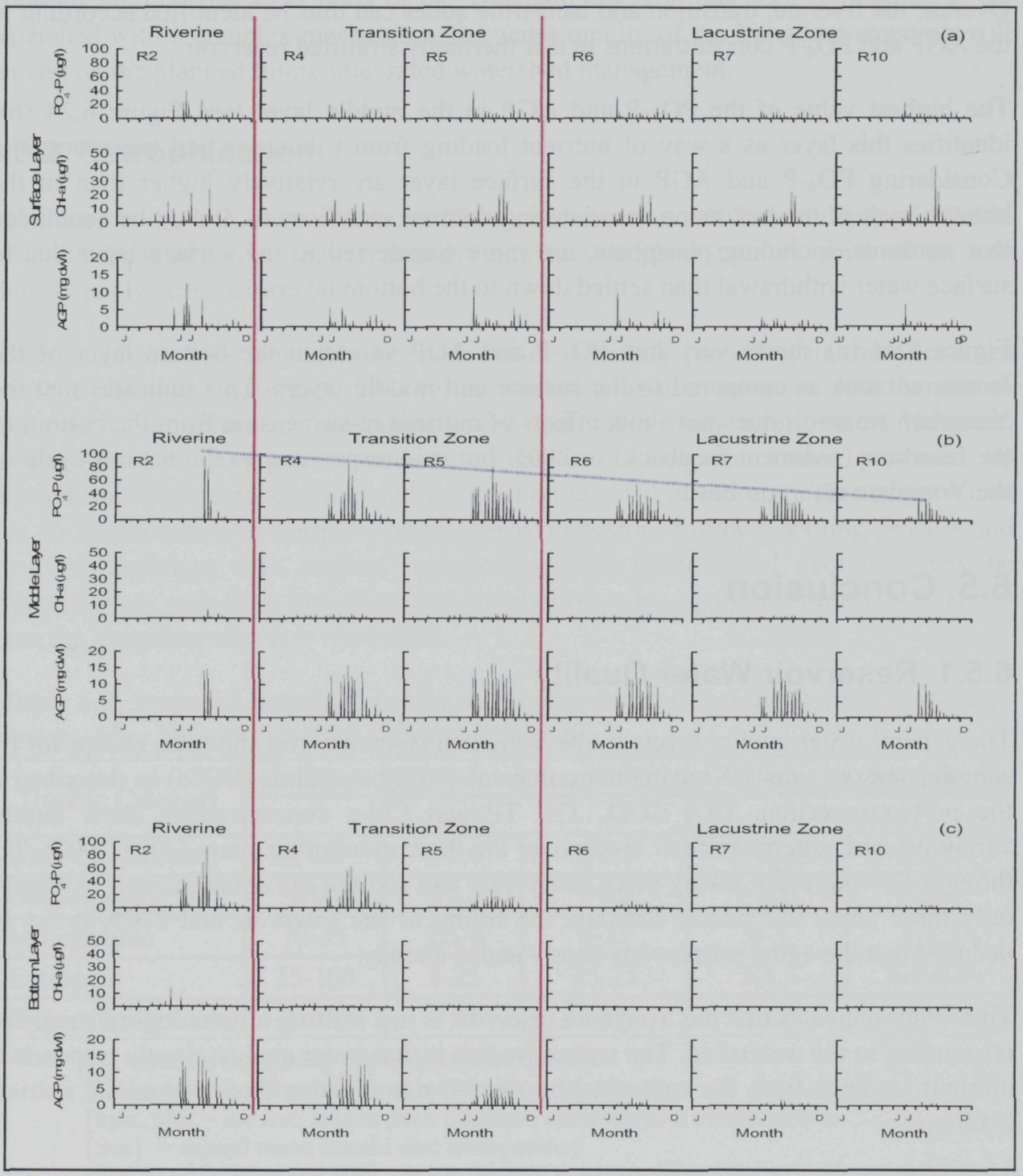

Figure 6.24. Spatial and temporal variations of $\mathrm{PO}_{4}-\mathrm{P}, \mathrm{Chl}-\mathrm{a}$ concentration and the AGP in the Yongdam Reservoir in 2005

Stations R6 to R10 (except for R8 and R9) have a dominant flow in the middle layer, i.e. a density flow due to density differences between the inflowing water and receiving water body. The Chl-a concentration in the surface layer is higher than that in the other layers; $\mathrm{PO}_{4}-\mathrm{P}$ concentrations and $\mathrm{AGP}$ are the highest in the middle layer during thermal stratification; the bottom layer can be considered as a stagnant water body. 
Overall, the riverine, transition and lacustrine zones can thus be identified according to the $\mathrm{AGP}$ and $\mathrm{PO}_{4}-\mathrm{P}$ concentrations in this thermally stratified reservoir.

The highest value of the $\mathrm{PO}_{4}-\mathrm{P}$ and $\mathrm{AGP}$ in the middle layer (see Figure 6.24 (b)) identifies this layer as a way of nutrient loading from tributaries and transportation. Considering $\mathrm{PO}_{4}-\mathrm{P}$ and $\mathrm{AGP}$ in the surface layer are relatively higher than in the bottom layer in the lacustrine zones during thermal stratification, it may be concluded that nutrients, including phosphate, are more transferred to the surface layer due to surface water withdrawal than settled down to the bottom layer.

Figure 6.24 (c) shows very low $\mathrm{PO}_{4}-\mathrm{P}$ and $\mathrm{AGP}$ values in the bottom layer of the lacustrine zone as compared to the surface and middle layers. This indicates that the Yongdam reservoir does not show effects of nutrient re-suspension from the bottom of the reservoir (sediment feedback) in 2005 , but it is responding to the natural cycle of the Yongdam reservoir basin.

\subsection{Conclusion}

\subsubsection{Reservoir Water Quality}

The general water quality levels in the Yongdam reservoir over the year, except for the summer season, satisfy Korean environmental quality standards (EQSs) as described in the previous section. DO, COD, TN, TP and Chl-a concentrations show similar variations and patterns in their levels over the three monitoring years (2005-2007). The through-flowing event taking place every year can shorten the aging process. Although only three years had passed between the filling of the reservoir and 2005, it can be deduced that the aging process has nearly ended already.

This study indicates that the Yongdam reservoir is just starting a limnological evolution responding to the watershed. The reason for this is that water quality closely responds to nutrient loadings from the watershed by rainfall-runoff rather than by internal nutrient loadings.

The local weather condition such as continuous rainy days and frequent rainfall causing soil erosion also influences the nutrient transportation, settling and the fate of pollutants in the reservoir through thermal stratification (single or double thermocline) of the reservoir water.

Since 2005, the COD and phosphorus loadings etc. have been decreasing over the three years, 2005-2007. The levels of pollutant in the upper reach are higher than lower reach in the surface layer except for Chl-a; this means that a deterioration of the reservoir water quality is developing from the upper reach to the lower reach. The density flows during thermal stratification in the middle layer changes the gradients of pollutants to the opposite direction from the surface layer. This means that. 
Consequently, for the water body of the Yongdam reservoir has been responding to the watershed with a complex mechanism, the water quality of the Yongdam reservoir will depend on the planned future integrated watershed management.

\subsubsection{Eutrophication}

According to the OECD standards for Chl-a concentration and Secchi depth (see Table 6.6), the Yongdam reservoir is already in a mesotrophic state, in spite of its low phosphorus concentration.

Eutrophication of the Yongdam reservoir water is mainly caused by nutrient loadings from the watershed, especially from spring rainfall to autumn turnover. And the pattern and level of eutrophication are similar every year without any deterioration. However, according to the analysis of sediment constituents, organic matter produced by eutrophication is being accumulated on the bottom of the reservoir. This gives a hint that an accumulation of nutrients on the reservoir bottom follow a settling process and form the sediment with various components. This limnological evolution may be repeated every year for a long time and largely driven by a pattern of nutrient loadings from the Yongdam reservoir watershed.

Table 6.6. Proposed boundary values for trophic categories (OECD fixed boundary system: after Vollenweider and Kerekes, 1980a, b)

\begin{tabular}{l|c|c|c|c|c}
\hline \multirow{2}{*}{ Trophic Category } & {$[\mathrm{P}]$} & {$[\mathrm{Chl}]$} & {$[\mathrm{max} . \mathrm{Chl}]$} & {$[\mathrm{Sec}]$} & {$[\mathrm{min} . \mathrm{Sec}]$} \\
\cline { 2 - 6 } & \multicolumn{4}{|c|}{$\mu \mathrm{g} / \mathrm{L}$} & \multicolumn{2}{|c}{$\mathrm{m}$} \\
\hline Ultra-oligotrophic & $\leq 4.0$ & $\leq 1.0$ & $\leq 2.5$ & $\geq 12.0$ & $\geq 6.0$ \\
\hline Oligotrophic & $\leq 10.0$ & $\leq 2.5$ & $\leq 8.0$ & $\geq 6.0$ & $\geq 3.0$ \\
\hline Mesotrophic & $10-35$ & $2.5-8$ & $8-25$ & $6-3$ & $3-1.5$ \\
\hline Eutrophic & $35-100$ & $8-25$ & $25-75$ & $3-1.5$ & $1.5-0.7$ \\
\hline Hypertrophic & $\geq 100$ & $\geq 25$ & $\geq 75$ & $\leq 1.5$ & $\leq 0.7$ \\
\hline
\end{tabular}

$[\mathrm{P}]=$ annual mean total phosphorus concentration

$[\mathrm{chl}]=$ mean ice free season chlorophyll a concentration

$[\max . \mathrm{chl}]=$ ice free season peak (highest) chlorophyll a concentration

[Sec] = annual mean Secchi disc transparency

$[\mathrm{min} . \mathrm{Sec}]=\operatorname{minimum}($ lowest $)$ Secchi disc transparency

\subsubsection{Algal Growth Potential Test}

The results of AGPT are discussed with the view point of limiting nutrients and spatial distributions of AGP in the thermally stratified Yongdam reservoir. According to the results, the Yongdam reservoir is a phosphate limited water body, except for the middle and the bottom layer in the upper reservoir for a short period in the summer.

The AGPT identifies that there are several unknown factors hindering algal growth in spite of bio-available nutrients existing in the surface layer of the Yongdam reservoir. 
The complex ecology of the reservoir makes it difficult to assess and predict the reservoir water quality. Therefore the AGPT is a good assessment method for reservoir eutrophication considering unknown factors.

The AGPT in the thermally stratified reservoir also shows nutrient loads and transport paths, and reservoir zones due to vertical and horizontal distributions of AGP over the entire reservoir. This is possible because of light limitation with depth for photosynthesis of phytoplankton in the water body, and because of density flows due to thermal stratification in the reservoir water body. The AGPT identified three distinct water layers (epilimnion, metalimnion and hyperlimnion), and three zones (riverine, R1-R3; transition, R4-R5 and lacustrine, R6-R10) in the Yongdam reservoir. It also showed that the surface water discharge of the Yongdam reservoir induces nutrient loadings from the middle water layer to the surface layer rather than settling down to the bottom of the reservoir. This nutrient transport mechanism can prevent nutrient accumulation on the bottom of the reservoir, but also offers an opportunity for a continuous algal production during thermal stratification and density flow.

\subsubsection{Interventions and Variables for Control of Eutrophication}

According to AGPT, the control variable of eutrophication for the Yongdam reservoir management is soluble reactive phosphorus (SRP, PO4-P). There are two possible interventions for the reduction of SRP in the reservoir: the reduction of the SRP load from the watershed and the abstraction and/or release of eutrophic water from the reservoir.

A general measure to delay eutrophication is the reduction of SRP load from the watershed. Figure 6.24 identifies the path of the nutrient load and transport, which can give an idea to abstract and/or release more eutrophicated water than ambient water. However, the abstraction and release of eutrophicated water may be limited because of the reservoir function for the alleviation of flood flows during the flood season in which nutrients are included, and the limited capacity of facilities for the abstraction and release of eutrophicated water. 


\section{Chapter 7}

\section{CASE STUDY: CONSTRUCTION OF MODELS}

\section{Summary}

Two and three dimensional physically-based models, data-driven models and a hybrid model combining the two categories of model (physically based and data driven) are applied separately in the case study which forms the focus of this Ph.D. research. The present study proposes a framework for hybrid modelling. The ecological water quality variables for deep and long reservoirs, like the Yongdam reservoir, can be successfully simulated using a two-dimensional model (in the vertical), but the modelling of eutrophication still has room for improvement. A three-dimensional model shows better simulation results for reservoir hydrodynamics than the two dimensional model despite it being developed for the ocean. The three-dimensional model as a management tool has a couple of short-comings in the amount of data required for water quality modelling and computing time. Data-driven models give simulation results based on instances; that is, measurements made at specific times and places. If the instance is not in the form of a regular time series, the models seek only a similar case from the historical data set. Actually, the time series database, representing the environmental processes in the reservoir, is difficult to develop. We can acquire only biweekly and monthly data because of budget and analysis limitations. As an alternative, we can use a physically-based model to provide time series simulation results at the time interval specified by modeler for time series data-driven modelling. As the eutrophication process has many unknown mechanisms, the Chl-a concentration may be predicted better with a data-driven model. The hybrid model and the corresponding prediction results of Chl-a concentrations show a better fit with observed data than the physically-based model alone. The results of the hybrid model can be used for a short-term forecasting of water quality in the reservoir and for long term prediction of the reservoir response to remedial activities affecting reservoir water quality. The hybrid model is subsequently implemented in a decision support system for water quality management in the Yongdam reservoir.

\subsection{Outline of Case Study}

The present case study started as a part of an integrated watershed research project for the Yongdam reservoir with the aim of developing a decision support system (DSS) as a management tool for water quality. There is often confusion concerning the terms 'basin', 'watershed' and 'catchment' (or 'drainage basin'). Here a watershed is the area of land where all surface and groundwater drains into the same body of water, such as a river, lake, or reservoir. A basin is regarded as a large scale watershed. A watershed is therefore a naturally delineated unit of land. In this sense, watersheds are the basic land unit of the hydrologic cycle. A catchment is the area of land that drains water, sediment, dissolved materials, and biota to a common outlet at some point along a stream channel. 
Briefly speaking, the catchment is the smallest unit in the watershed. Watersheds are regarded as natural containers that allow us to understand our environment better. Thus the research at the watershed level of the Yongdam reservoir includes all the research activities as described in Figure 1.5. However, this study is limited primarily to the water body of the Yongdam reservoir.

The modelling approach adopted in this study is shown in Figure 7.1. Both physicallybased and data-driven models are instantiated using the data produced for this study and through management activities, and finally the two kinds of model are integrated in the so-called hybrid model.

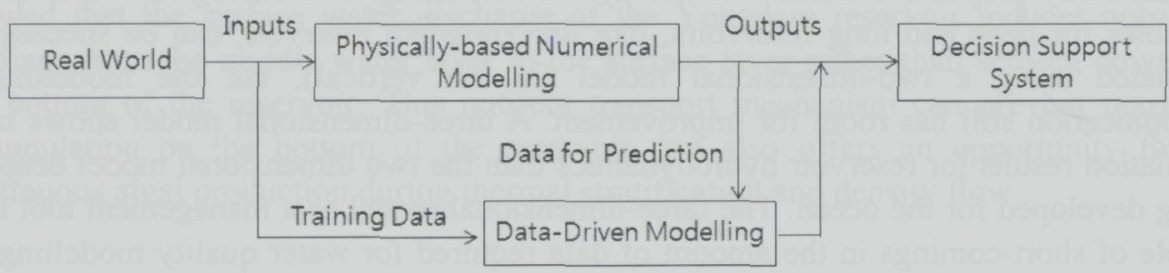

Figure 7.1. General schemes of hybrid model in DSS.

The physically-based models are constructed to interpret global mixing and nutrient transport, and the new approach of data-driven modelling is implemented to mine information and to predict environmental processes such as eutrophication and biochemical reactions in the reservoir water body. Many environmental data are needed in order to analyze relationships between parameters for short-term forecasting purposes and/or to get information which is not included in a physically-based model due to unidentified mechanisms.

Data-driven modelling uses statistics and artificial intelligence techniques to discern an individual pattern embedded in the data set. For the data-driven model, which is essentially a time series model, the data used for model construction must have time intervals that are appropriate to describe in sufficient detail the processes, including the bio-chemical reactions among the constituent parameters.

The physically-based models can generate time series data which are many times impossible to obtain through measurements due to the large budget required for sampling and analysis. A data-driven model can reduce the uncertainty in the results from the physically-based model by replacing that part of the simulation processes in the physically-based model which are poorly defined. Therefore the integration of physically-based and data-driven models has the potential to give more accurate time series simulation results to decision makers.

Finally, the hybrid model can be a valuable decision support tool for short-term forecasting and prediction of reservoir behavior due to environmental changes at the watershed level. 


\subsection{Eco-hydrodynamic Modelling}

\subsubsection{Model Selection}

The principal step in any model study is the selection of a model that is appropriate in representing the domain and processes under consideration. In this study, two representative models, MIKE 3D and CE-QUAL-W2 were selected from the available modelling software introduced in ANNEX I. The following reasons give proof that both models can be applied to the Yongdam reservoir:

- They require a similar level of data, and solve the equations of the laws of conservation: continuity equation, constituents transport equation, the Navier-Stokes equations for mass, momentum and energy, and equations of state etc.

- They deal with turbulence, such as using a related viscosity such as a constant eddy viscosity, Smagorinsky formulation, $k$-model formulation, $k-\varepsilon$ formulation, and mixed $k-\varepsilon /$ Smagorinsky formulation etc.; the two models have an option to select a turbulent model.

- They adopt one of several time variable solutions such as CDS, UDS, QUICK, QUICKEST, and TVD etc. (the TVD scheme has been developed comparatively recently, and it is not commonly applied at present).

- They use a particular approach to solve the partial differential equations with computational grids such as finite differences or finite volumes.

\section{1) CE-QUAL-W2}

The CE-QUAL-W2 model has been successfully applied in numerous case studies, ranging from steeply sloping rivers to deep reservoirs and narrow estuaries. This model has been under development at the US Army Corps of Engineers since 1974 and has undergone extensive review and testing by Johnson (1981). Previous verification studies using LARM (Laterally Averaged Reservoir Model), GLVHT (Generalized Longitudinal-Vertical Hydrodynamics and Transport Model), and CE-QUAL-W2 that was developed by expanding LARM have been presented by Gordon (1980, 1981 and 1983); Edinger et al. (1983); Kim et al. (1983); and Martin (1988). The successful testing of a two-dimensional hydrothermal and hydrodynamic model for a reservoir is documented by Gelda and Effler (2007). Mass-balance based eutrophication simulation models are commonly used tools for predicting the ecological response of a surface water body to potential changes in anthropogenic nutrient loading (Arega and Lee, 2000) under existing conditions and for various levels of nutrient load reduction. For this study, CE-QUAL-W2 has priority, in view of the experience of applying the model to long, deep reservoirs, its ability to model turbid density flows in stratified reservoirs, and the continuous improvement that has been made to the model.

CE-QUAL-W2 uses a staggered grid to solve the six governing equations for the six unknowns, namely: horizontal (i.e., along-axis) velocity, vertical velocity, free water 
surface elevation, constituent concentration, pressure, and density. These variables are governed by the laterally-averaged version of the continuity equation, the horizontal $(x)$ momentum equation, the vertical $(z)$ momentum equation, state equation, and the free water surface elevation equation.

One of the principal motivations for the use of a two-dimensional model which focuses on the flow associated with the vertical coordinate is to investigate the vertical temperature and density structure as well as vertical mixing. Therefore, it is important to incorporate turbulence into CE-QUALW2. As outlined in Cole \& Buchak (1995), it is assumed that the horizontal flow variables may be written as:

$$
\begin{aligned}
& u=U+u^{\prime}+u^{\prime \prime} \\
& w=W+w^{\prime}+w^{\prime \prime}
\end{aligned}
$$

in which $u$ and $w$ are the instantaneous velocities; $U$ and $W$ are the time averaged mean velocities; $u^{\prime}$ and $w^{\prime}$ are the turbulent velocity fluctuations about the mean generated by the shear; and $u^{\prime \prime}$ and $w^{\prime \prime}$ are the velocity components of wind waves propagated downward from the water surface. CE-QUAL-W2 uses a first order scheme to represent turbulent transport, which is written as $\left(u^{\prime} w\right)=-A_{z}(\partial U / \partial z)$ where $A_{z}$ is the vertical eddy viscosity. Both the along-axis shear (associated with the $u$-direction) and lateral shear (associated with the $v$-direction) associated with surface winds are assumed to decay exponentially with distance from the surface as:

$$
\begin{aligned}
& \left\langle u^{\prime \prime} w^{\prime \prime}\right\rangle=\tau_{w x} e^{-2 k z} \\
& \left\langle v^{\prime \prime} w^{\prime \prime}\right\rangle=\tau_{w y} e^{-2 k z}
\end{aligned}
$$

Here $\tau_{w x}\left(\tau_{w y}\right)$ is the surface shear resulting from along-axis wind waves of wave number $k$. Thus the horizontal shear is assumed to be of the form:

$$
\tau_{x}=-A_{z} \frac{\partial U}{\partial z}+\tau_{w x} e^{-2 k z}
$$

Finally, the vertical eddy viscosity coefficient $A_{z}$ is formulated as:

$$
A_{z}=K\left(\frac{L^{2}}{2}\right) \sqrt{\left(\frac{\partial U}{\partial z}\right)^{2}+\left(\frac{\partial V}{\partial z}\right)^{2}} e^{\left(-C R_{i}\right)}
$$

in which $K$ is the Von Karman constant with a value of $0.4 ; L$ is a vertical length scale taken as $\Delta z ; C$ is a constant taken as $1.5 ; R_{i}$ is the local Richardson number; and $V$ is the lateral velocity. When the laterally averaged velocity, $V$, and its vertical gradient disappear, the term $(\partial \mathrm{V} / \partial \mathrm{z})^{2}$ does not necessarily vanish and has to be represented as: 


$$
\left(A_{z} \frac{\partial V}{\partial z}\right)^{2}=\left(\tau_{w y} e^{-k z}\right)^{2}
$$

The substitution of Equation (7.7) into Equation (7.6) leads to an implicit representation for the vertical eddy viscosity coefficient, $A_{z}$. When solved numerically, the value for $A_{z}$ from the previous time step is used to calculate Equation (7.7) for the square of the vertical shear, which is then used in Equation (7.6) to update the value of $A_{z}$. This formulation thus parameterizes vertical turbulent mixing for the shear in the mean velocity profile and for velocity fluctuations resulting from wind waves, and accounts for the damping effect of density stratification on turbulence through the Richardson number. This formulation is standard in hydrodynamic modelling (Kowalik \& Murty, 1993).

The formulation of an algal community in CE-QUAL-W2 gives the user complete freedom on how many and what kinds of algal groups can be included in the simulation. Figure 7.2 presents the principal kinetic interactions for nutrient cycles and algae. This is done through careful specification of the kinetic rate parameters that define the characteristics of each algal group. The rate equation for each algal group is:

$$
S_{a}=\underbrace{K_{a g} \Phi_{\mathrm{a}}}_{\text {growth }}-\underbrace{K_{a r} \Phi_{a}}_{\text {respiratio }}-\underbrace{K_{a e} \Phi_{a}}_{\text {excretion }}-\underbrace{K_{a m} \Phi_{a}}_{\text {mortality }}-\underbrace{\omega_{a} \frac{\partial \Phi_{a}}{\partial z}}_{\text {settling }}
$$

in which $K_{a g}, K_{a r}, K_{a e}$ and $K_{a m}$, are the algal rate for growth, respiration, excretion and mortality with the unit of $\left[\mathrm{T}^{-1}\right] ; \omega_{a}$ is the algal settling rate $\left[\mathrm{LT}_{-1}\right]$; and $\Phi_{a}$ is the algal concentration $\left[\mathrm{ML}^{-3}\right]$. Algal growth rate is computed by modifying a maximum growth rate affected by temperature, light, and nutrient availability.

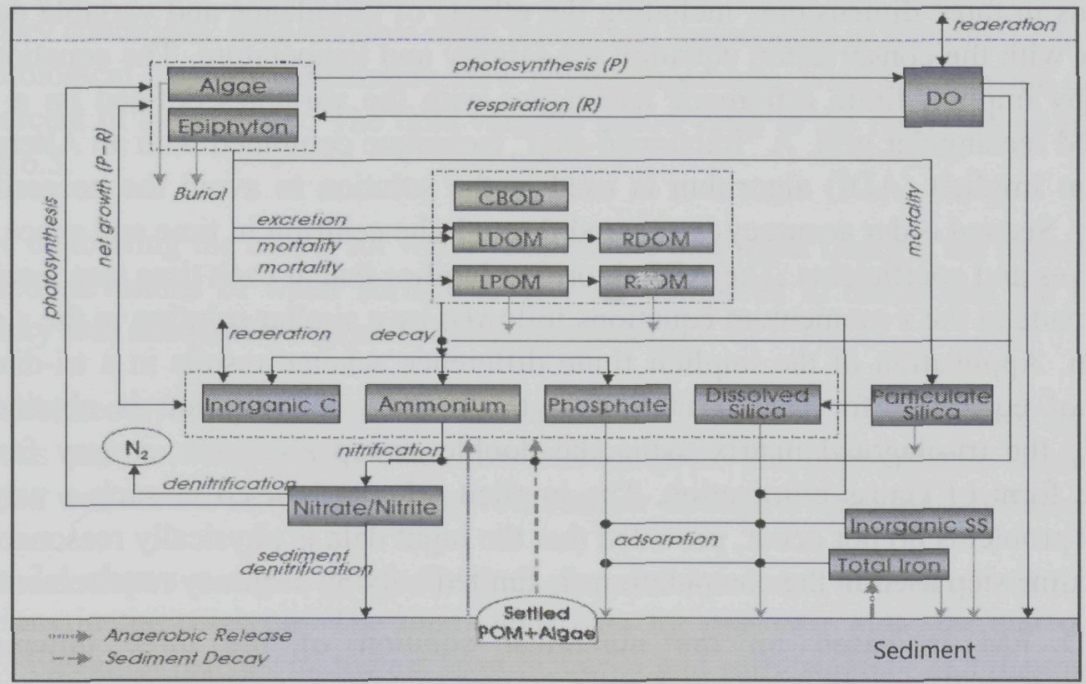

Figure 7.2. Interactions among constituents in CE-QUAL-W2 (KNIER, 2006). 


\section{2) MIKE 3}

MIKE 3, like most three dimensional models, was developed primarily for the ocean (estuary) modeling and then extended for lakes and reservoirs. It was selected for the following reasons:

- It simulates unsteady flow taking into account density variations, bathymetry and external forcing such as meteorology, tidal elevations, currents and other hydrographic conditions.

- The effect of mixing due to waves on currents can be included in various ways e.g. due to wind at the surface and inflows at the thermocline. Inclusion of wave-induced mixing in the model is done by specifying wave radiation stresses that will enter the momentum equations. These functions are important in reservoirs.

In the latest decades numerical models have been developed, corresponding to the Computational Fluid Dynamics (CFD). So MIKE 3 also has two kinds of numerical models: Finite Difference Methods (FDM) on structured grids (MIKE 3D) and Finite Volume Methods (FVM) on unstructured mesh (MIKE 3D FM). The two models are tested in this study.

In most three-dimensional models the fluid is assumed incompressible. However, using the divergence-free (incompressible) mass equation, the set of equations will inevitably form a mathematically ill-conditioned problem because the fluid pressure does not appear in the divergence-free mass equation, which causes a weak coupling between the pressure and velocities. This is a key issue in three-dimensional modelling. MIKE 3D uses the artificial compressibility method to solve this problem, whereby the pressure is replaced by information about the surface elevation. The mathematical basis of MIKE $3 \mathrm{D}$ consists of the mass conservation equation, the Reynolds-averaged Navier-Stokes equations in three dimensions, including the effects of turbulence and variable density, together with the conservation equation for salinity and temperature. The equations are solved by implicit finite difference techniques with the variables defined on a space staggered rectangular grid. A "fractioned-step" technique combined with an Alternating Direction Implicit (ADI) algorithm is used in the solution to avoid the necessity for iteration. Second order accuracy is ensured through the centring in time and space of all derivatives and coefficients. The ADI algorithm implies that at each time step a solution is first made in the $x$ momentum equations followed by a similar solution in the $y$ and $z$ direction. Application of the implicit finite difference scheme results in a tri-diagonal system of equations for each grid line in the model. The solution is obtained by inverting the tri-diagonal matrix using the double sweep algorithm, a very fast and accurate form of Gauss elimination. The implicit scheme is used in such a way that stability problems do not occur, provided that the input data is physically reasonable, so that the time step used in the computations is limited only by accuracy requirements.

MIKE3D FM is based on the numerical solution of the three dimensional incompressible Reynolds averaged Navier-Stokes equations invoking the assumptions of Boussinesq and of hydrostatic pressure. Thus, the model consists of continuity, 
momentum, temperature, salinity and density equations and it is closed by a turbulent closure scheme. For the 3D model the free surface is taken into account using a sigmacoordinate transformation approach.

\subsubsection{Instantiating Eco-Hydrodynamic Models for Yongdam Reservoir}

\section{1) CE-QUAL-W2}

\section{a) Construction and Calibration}

Applying the CE-QUAL-W2 modeling framework assumes that a water system is segmented into a number of completely mixed water cells. An iterative process has been implemented to determine the proper segmentation of the reservoir based on the bottom bathymetry.

The Yongdam reservoir is configured as a three-branch system according to the shape of each branch and the distribution of the tributaries, and is schematized to consist of 58 longitudinal segments at the surface with lengths between $250.38 \mathrm{~m}$ and $890.37 \mathrm{~m}$. Each segment is then divided into $1 \mathrm{~m}$ layers in the water column. The entire water column is thus configured with a total of 1,780 segments. Figure 7.3 shows the model grid for the Yongdam reservoir. Time steps of 50 seconds to 1 hour were adopted by the model simulations for the reservoir due to different inflow conditions (during the flood and dry season).

The hydrodynamic model was first calibrated with the 2005 data for the Yongdam reservoir. The comprehensive input data for CE-QUAL-W2 hydrodynamic modeling includes reservoir topography, meteorological, inflow and outflow of reservoir, flow and temperature of stream information. The driving force for this model includes: boundary conditions: time-variable main inflow and temperature at Stations T1 to T5, and meteorological data: air temperature, dew point temperature, wind speed, wind direction, and percent of cloud cover. The time variable inflow rates to the reservoir are plotted in Figure 6.5.

Before calibrating the model for water temperature and water quality, the measured and modeled elevations of water surface level were compared to check that the reservoir geometry was adequately represented by the model.

The calibrated hydrodynamic model was validated for the flows, the meteorology forcing functions and the water quality data measured for 2006. The time-variable water surface elevation was used to estimate the reservoir outflow through a water mass balance.

Figure 7.4 shows a close match between the simulated and measured water surface elevations in the reservoir. The time series data for one year indicates that the water surface elevation reaches its lowest level in spring and early summer and then gradually rises and reaches a peak in late summer and autumn during periods of typhoons and thunderstorms. 


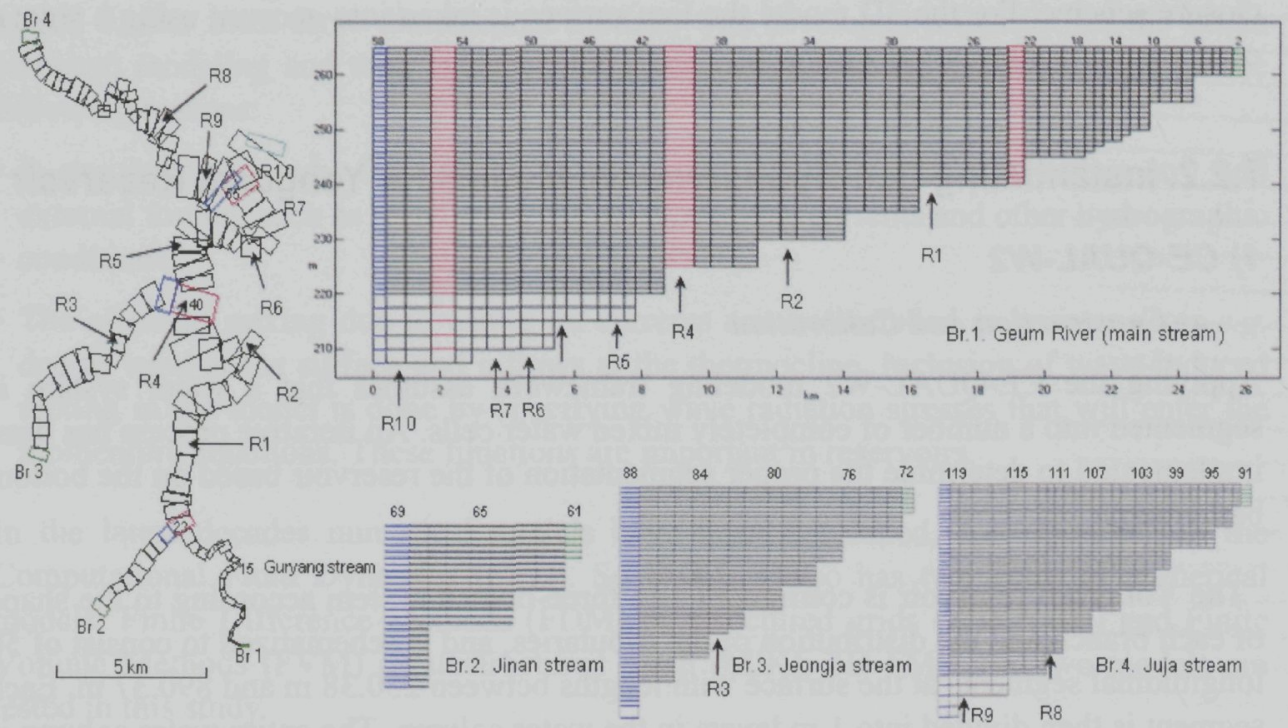

Figure 7.3. Discretized grids of the Yongdam reservoir and Sampling stations.

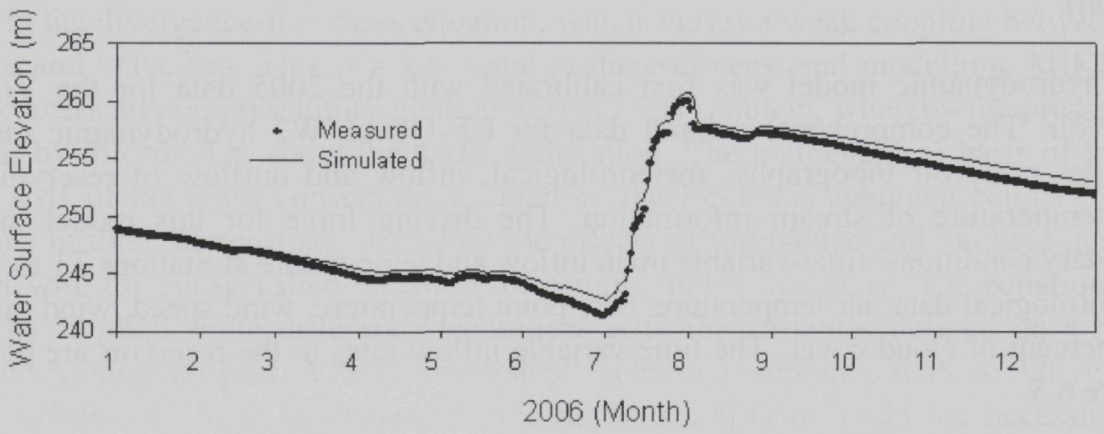

Figure 7.4. Modeled and Measured Water Surface Elevation and Discharges in 2006. (Blue dotted line: Simulation data and red real line: Measured data)

Water temperature data were used to evaluate the hydrodynamic results for the year 2006. These results are plotted in Figure 7.5 and 7.6. The Yongdam reservoir exhibits a pronounced vertical thermal gradient in the summer and undergoes significant overturning by the end of the autumn in each year.

Strong thermal stratification occurs from May to October; see the data for the year 2006 in Figure 7.5, which shows that the reservoir water maintains the same temperature in depth from January to early April, and then goes through thermal stratification and destratification during the remainder of the year. 

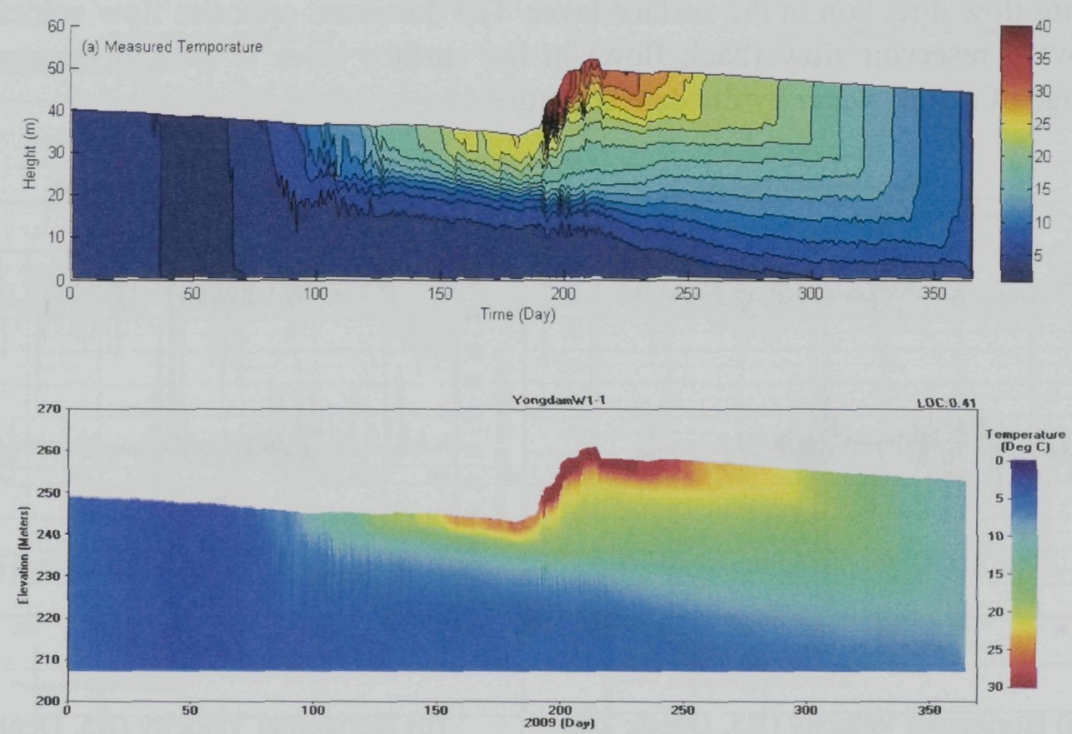

Figure 7.5. Time series vertical Temperature profile at R10 in 2006: (a) Measured data and (b) Simulation data by CE-QUAL-W2.

Temperature $\left({ }^{\circ} \mathrm{C}\right)$
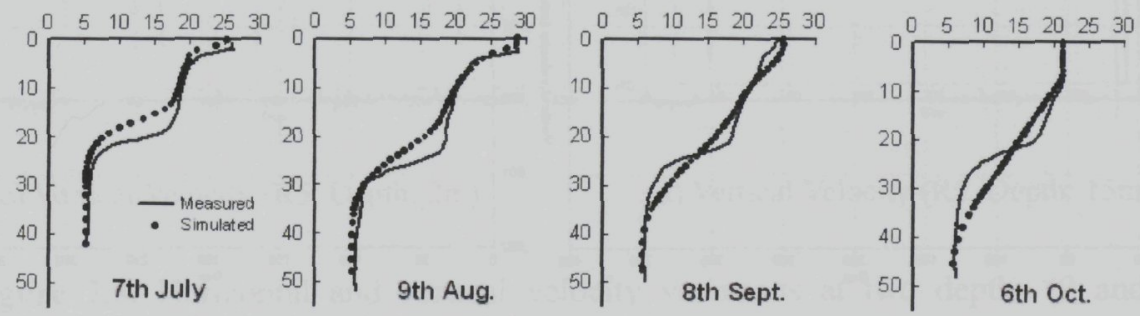

Figure 7.6. Vertical Temperature distribution in CE-QUAL-W2 at R10 in 2006.

According to the results of the AGPT in Chapter 6, the flow regime of Yongdam reservoir consists of a riverine zone, which spreads from the three tributaries to R4, through a transition zone R5, to a lacustrine zone which starts at R6.

The results of CE-QUAL-W2 simulation are also figured out the horizontal and vertical flow velocities to represent forcing function in the surface and middle layers along the main stream of the reservoir.

\section{b) Simulation Results for Horizontal and Vertical Velocity}

According to Figure 7.7, the flow at R2 shows a typical river flow that the horizontal flow forms a streamline in the bottom layer without nearly vertical mixing and a 
opposite flow direction in the surface layer. The dominant opposite flow velocity to the downward reservoir flow (back flow) in the surface layer is formed because of the blockage of inflow water by the dam structure.

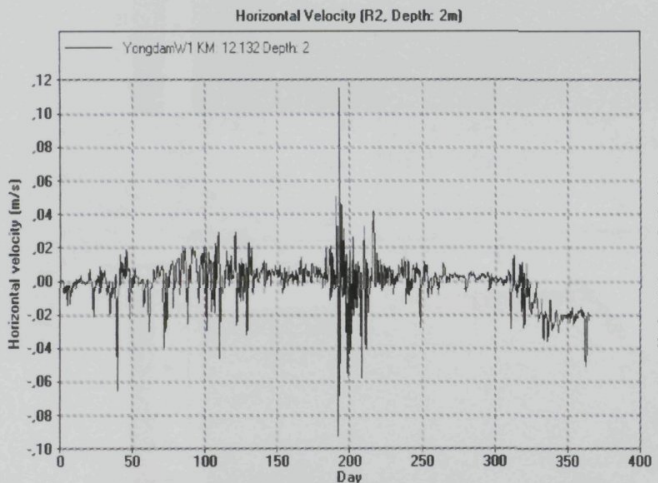

(a) Horizontal Velocity (R5, Depth: 2m)

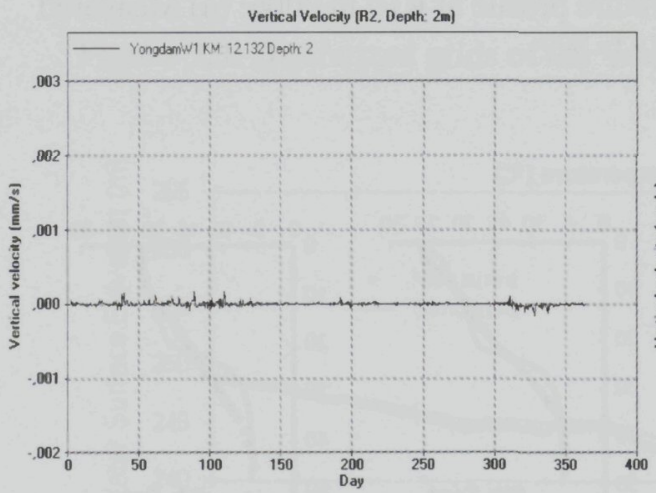

(c) Vertical Velocity (R5, Depth: 2m)

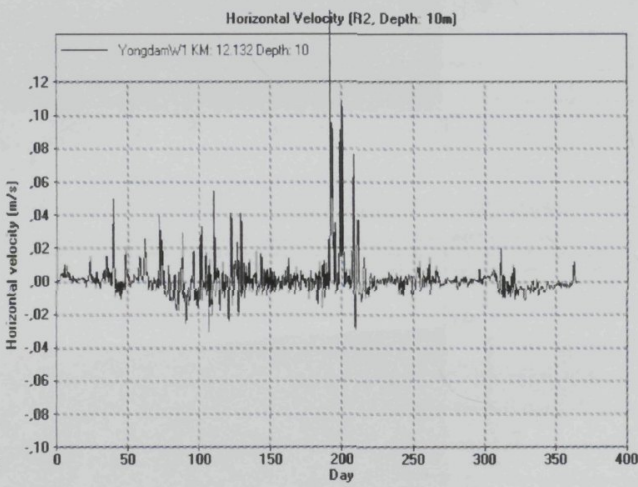

(b) Horizontal Velocity (R5, Depth: 10m)

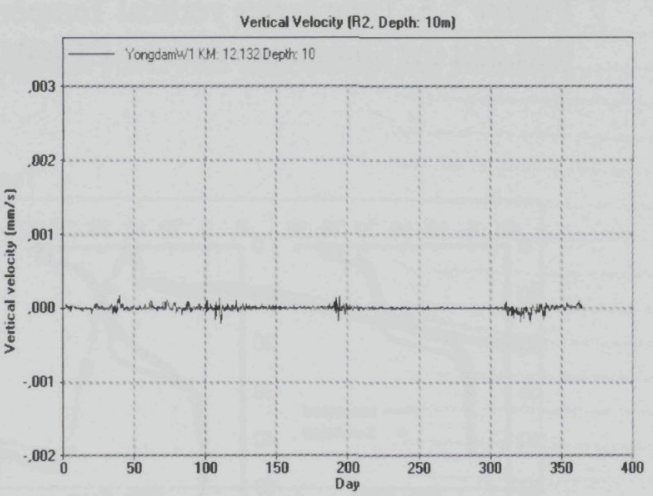

(d) Vertical Velocity (R5, Depth: 10m)

Figure 7.7. Horizontal and vertical velocity variations at two depths (2 and 15 meters) of R2 in 2006.

According to Figure 7.8 , the vertical flow at R4 shows a different variation from R2. Jeongja stream (T4) affects the great fluctuation of the vertical velocity in the water column at R4.

As the water depth of R4 is deepening, the vertical mixing due to different water density from three flow regimes (a downstream and two upstream) takes place and then the water column is stratified according to the water density. This region in the reservoir called the transition zone. 


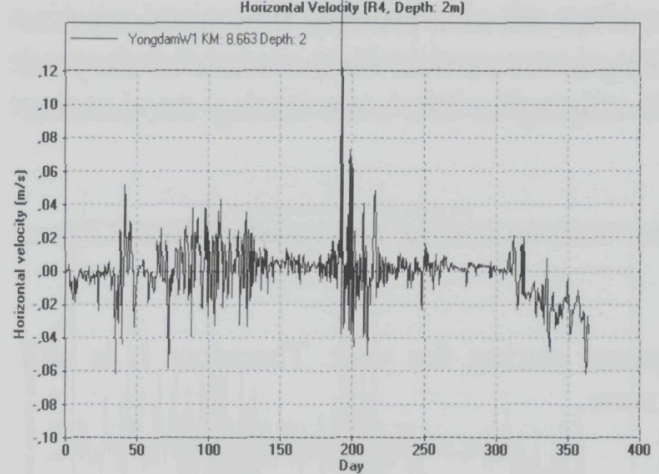

a) Horizontal Velocity (R5, Depth: 2m)

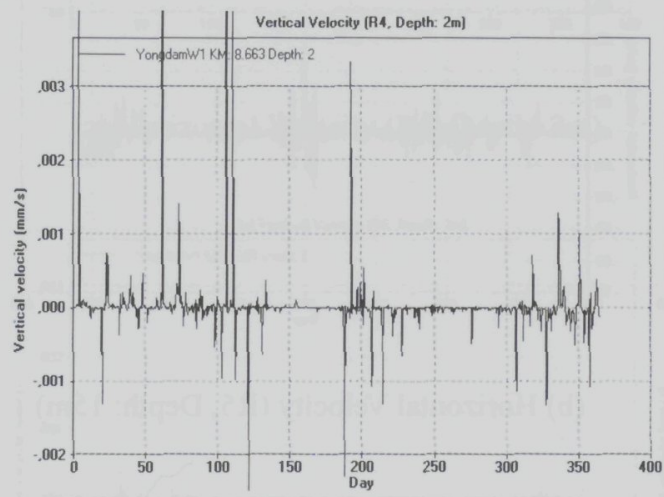

(c) Vertical Velocity (R5, Depth: 2m)

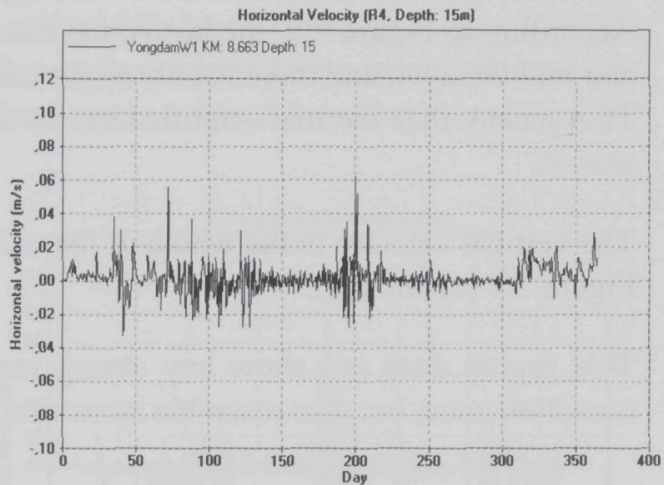

(b) Horizontal Velocity (R5, Depth: 15m)

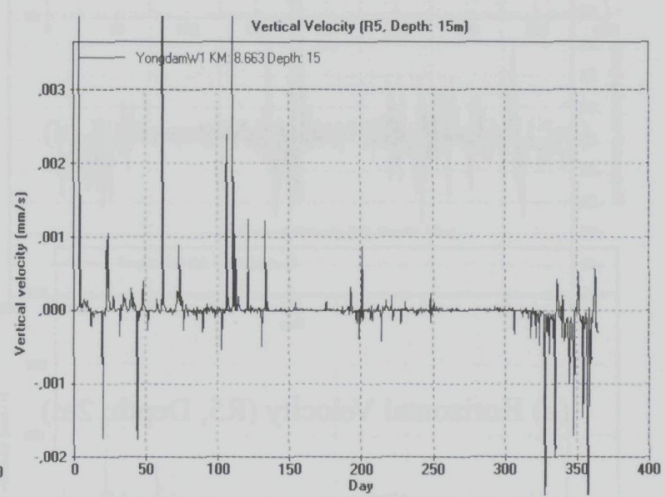

(d) Vertical Velocity (R5, Depth: 15m)

Figure 7.8. Horizontal and vertical velocity variations at two depths ( 2 and 15 meters) of R4 in 2006. 
The R5 is also the transition zone according to the AGPT in Chapter 6 (see Figure 6.19). According to Figure 7.9, the horizontal flow at R5 shows a primary flow in the surface and middle layer, and there is only slight mixing in the vertical for the whole of the year. This means that thermal stratification is developing in this zone during the summer season.

The vertical velocity in the whole of the water column is slow and constant like R2 (see Figure 7.7 (c) and (d)).

This region does not show any strong turnover during the year. Therefore it is still transition zone, but also resemble lacustrine zone.

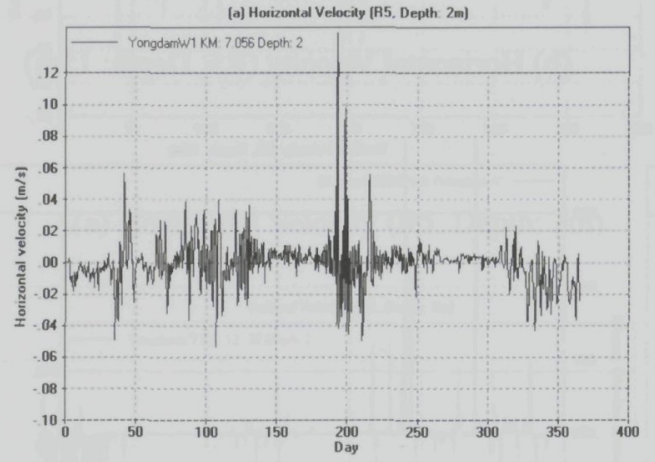

(a) Horizontal Velocity (R5, Depth: 2m)

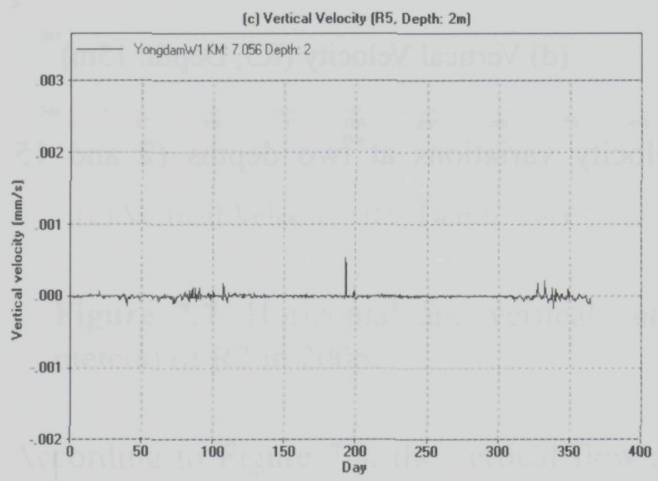

(c) Vertical Velocity (R5, Depth: 2m)

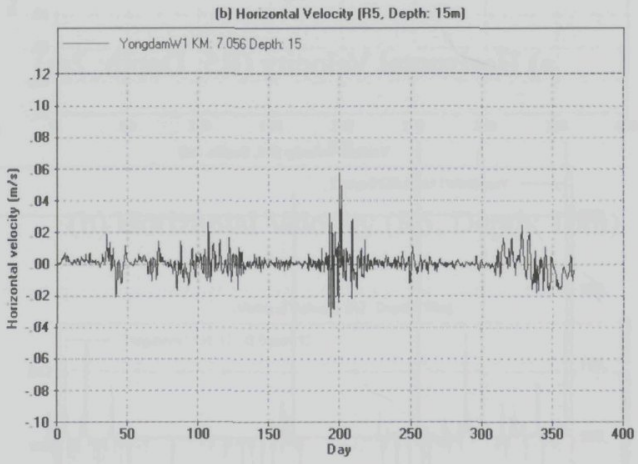

(b) Horizontal Velocity (R5, Depth: 15m)

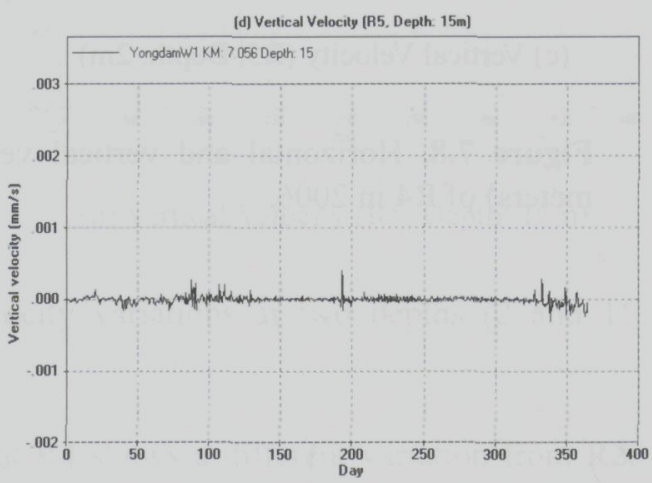

(d) Vertical Velocity (R5, Depth: 15m)

Figure 7.9. Horizontal and vertical velocity variations at two depths ( 2 and 15 meters) of R5 in 2006. 
According to Figure 7.10, R6 still has the characteristics of a transition zone because of a similar horizontal flow to R5 in the surface and middle layer. However Figure 7.10 (d) shows that there are vertical turnovers in spring and autumn. The horizontal flow at the surface layer is dominant, but the flow direction repeats a forward and backward flow.

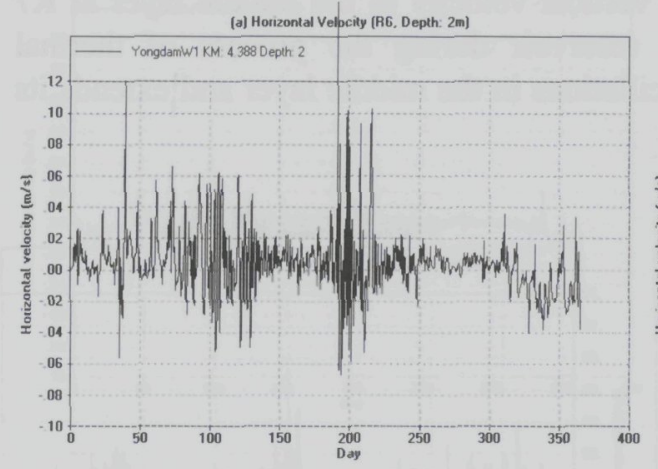

(a) Horizontal Velocity (R6, Depth: 2m)

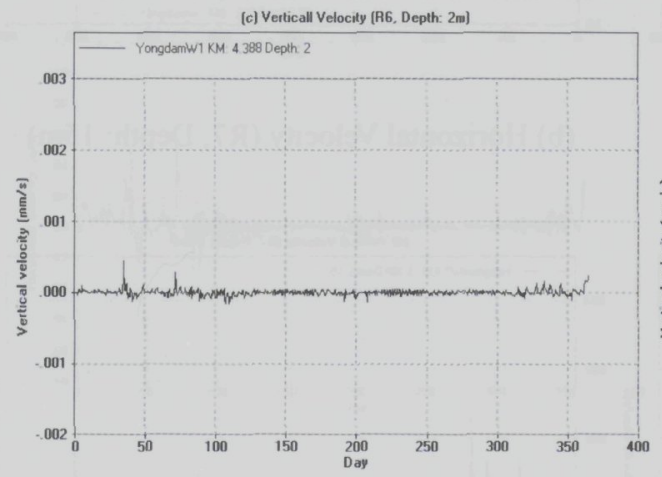

(c) Vertical Velocity (R6, Depth: 2m)

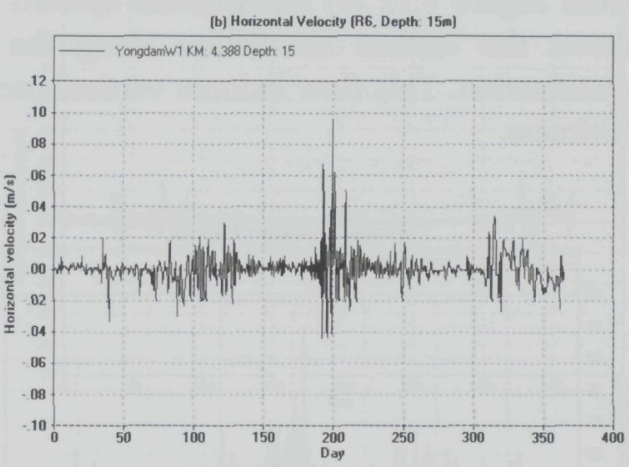

(b) Horizontal Velocity (R6, Depth: 15m)

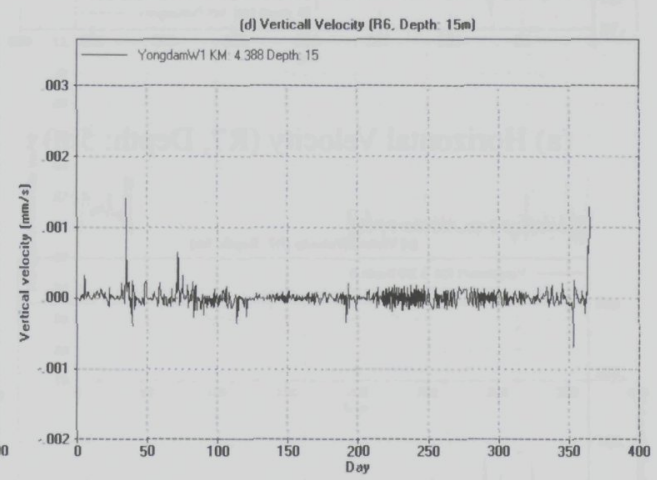

(d) Vertical Velocity (R6, Depth: 15m)

Figure 7.10. Horizontal and vertical velocity variations at two depths ( 2 and 15 meters) of R6 in 2006. 
The zone R7 is the lacustrine zone according to Figure 7.11. The vertical mixing velocity in the middle layer is faster than in the surface layer, whose main direction is upward in the spring and late autumn. The horizontal velocity in the surface layer is influenced by the dam structure (see Figure 7.11 (a): back flow in the spring).

From Figure 7.11 (c) and (d), the upward vertical velocity in the middle layer at R7 shows the vertical circulation along the reservoir during the process of thermal stratification. This flow induces vertical oscillations in the middle layer and extends its thickness.

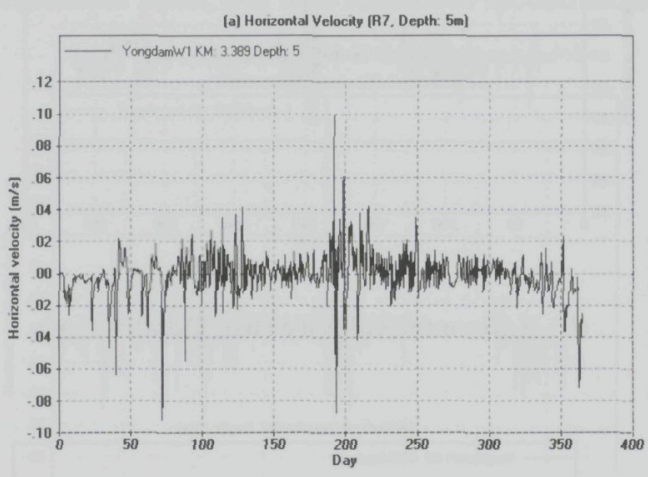

(a) Horizontal Velocity (R7, Depth: $5 \mathrm{~m})$

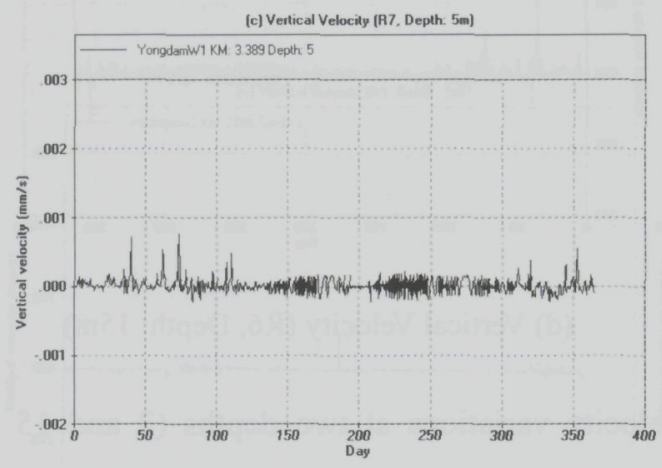

(c) Vertical Velocity (R7, Depth: 5m)

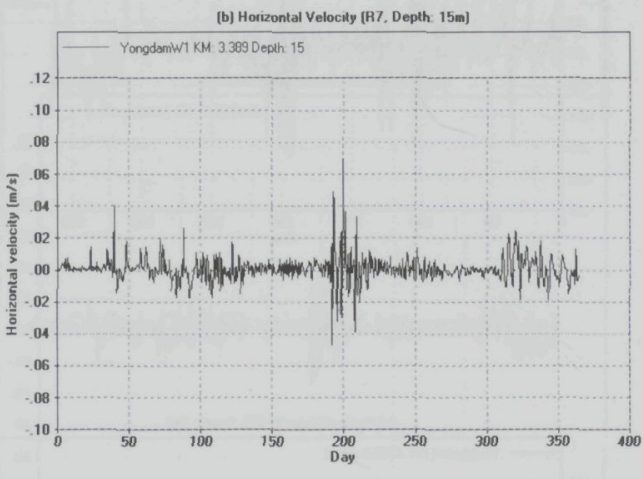

(b) Horizontal Velocity (R7, Depth: 15m)

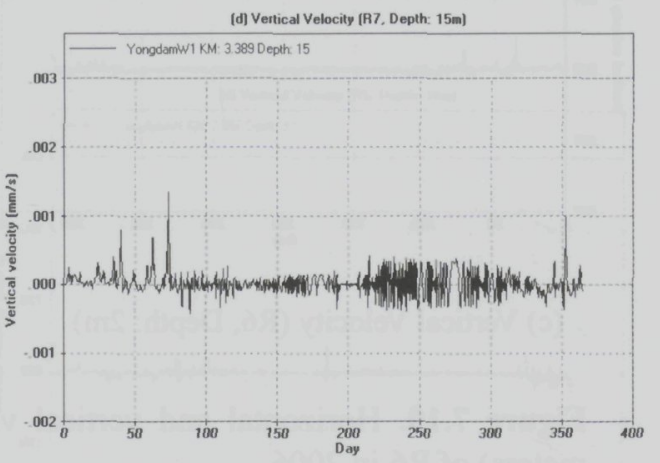

(d) Vertical Velocity (R7, Depth: 15m)

Figure 7.11. Horizontal and vertical velocity variations at two depths (5 and 15 meters) of R7 in 2006. 
According to Figure 7.12, the horizontal velocities at R10 (near the dam site) are dominant forward in the middle layer and backward flow in the surface layer because of the dam structure hindering further flow in the middle layer.

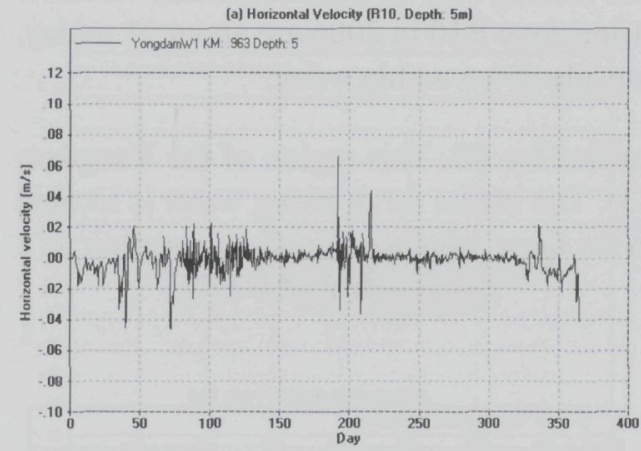

(a) Horizontal Velocity (R10, Depth: 5m)

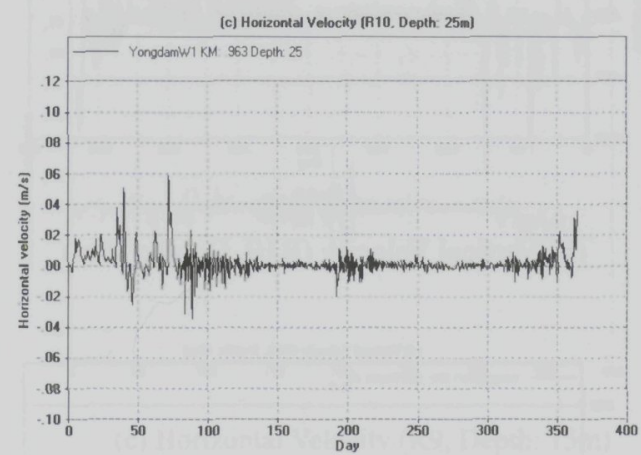

(c) Horizontal Velocity (R10, Depth: 25m)

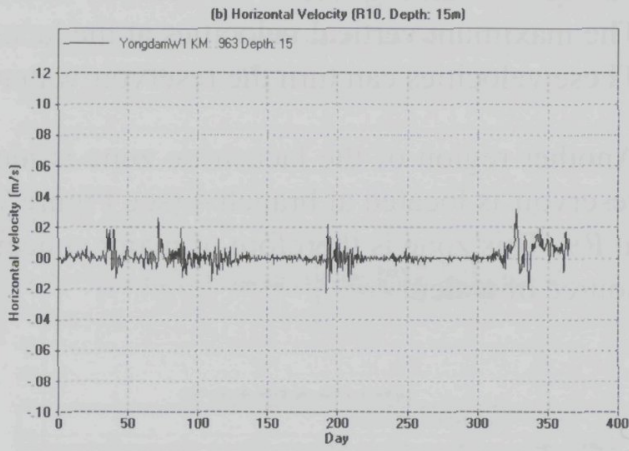

(b) Horizontal Velocity (R10, Depth: 15m)

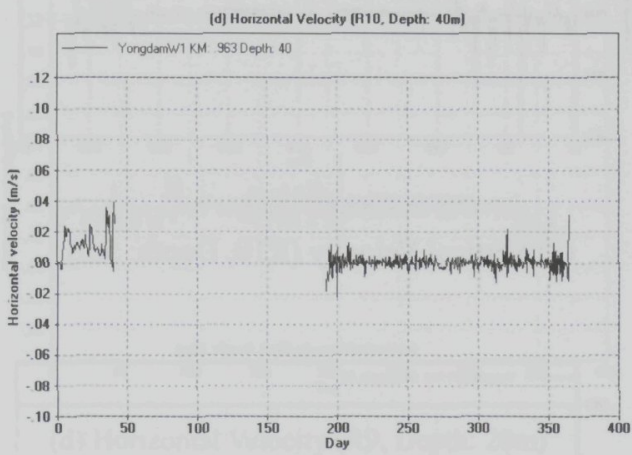

(d) Horizontal Velocity (R10, Depth: 40m)

Figure 7.12. Horizontal velocity variations at four depths $(5,15,25$ and 40 meters) of R10 in 2006. 
The downward vertical velocities at R10 (Figure 7.13) are different from Figure 7.11 and identify the vertical circulation along the reservoir described above.

According to Figures 7.11 and 13 , in the lacustrine zone the vertical mixing velocity during overturning due to differences of water density can be significant and variable. The maximum vertical velocities at the lacustrine zone R10 in 2006 are about $91 \mathrm{~m} /$ day. These velocities can turn the reservoir water upside down within a day.

Another region of the lacustrine zone is identified by R9. This region of the Yongdam reservoir is located at branch 4 (see Figure 7.3). The intake for drinking water is located at R9. This zone is therefore of major concern for those who use Yongdam reservoir as a source of water.

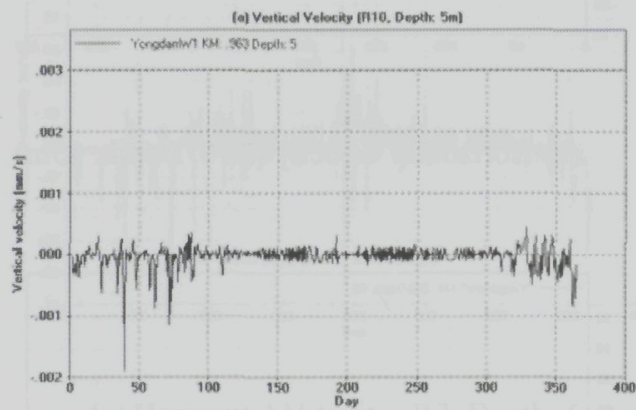

(a) Vertical Velocity (R10, Depth: 5m)

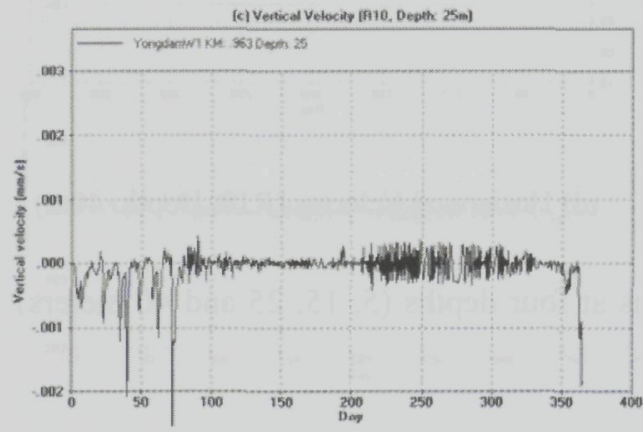

(c) Vertical Velocity (R10, Depth: 25m)

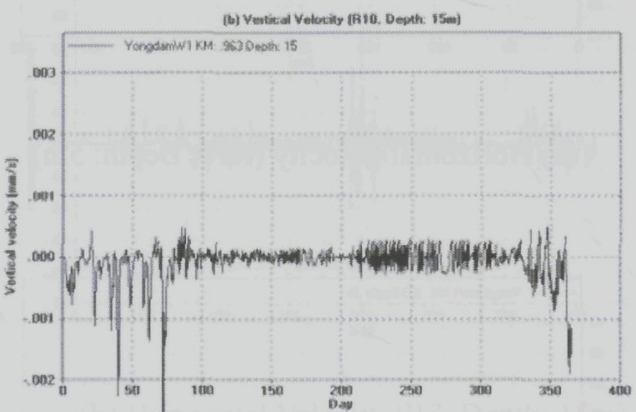

(b) Vertical Velocity (R10, Depth: 15m)

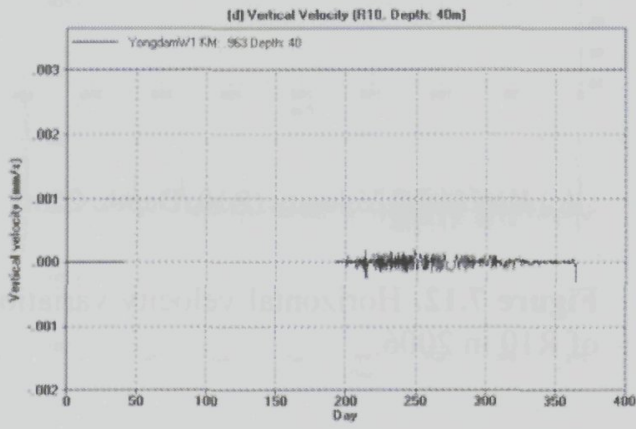

(d) Vertical Velocity (R10, Depth: 40m)

Figure 7.13. Vertical velocity variations at four depths $(5,15,25$ and 40 meters) of $\mathrm{R} 10$ in 2006. 
The flow regime at $\mathrm{R} 9$ is quite different from $\mathrm{R} 10$. The main cause of the horizontal flow, except for the spillway discharge, is due to the intake at R9 with the usual abstraction depth of the intake tower at around 10 meters. The horizontal flow takes place in the upper layers (see Figure 7.14).

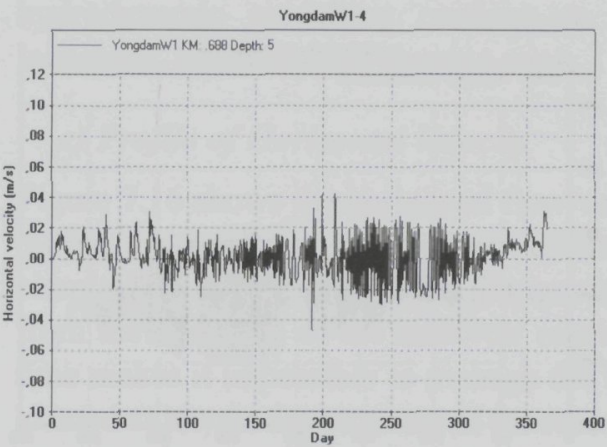

(a) Horizontal Velocity (R9, Depth: $5 \mathrm{~m}$ )

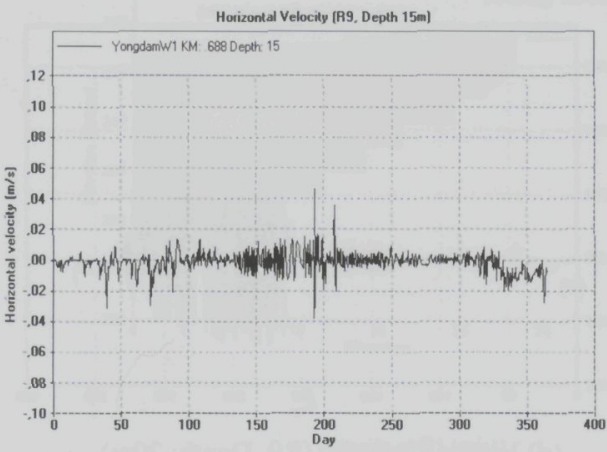

(c) Horizontal Velocity (R9, Depth: 15m)

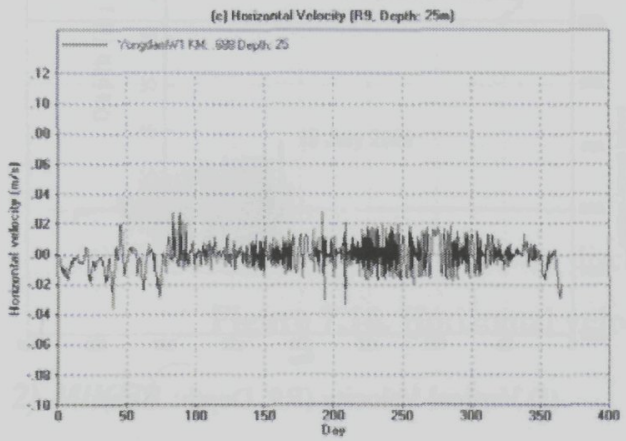

(e) Horizontal Velocity (R9, Depth: 25m)

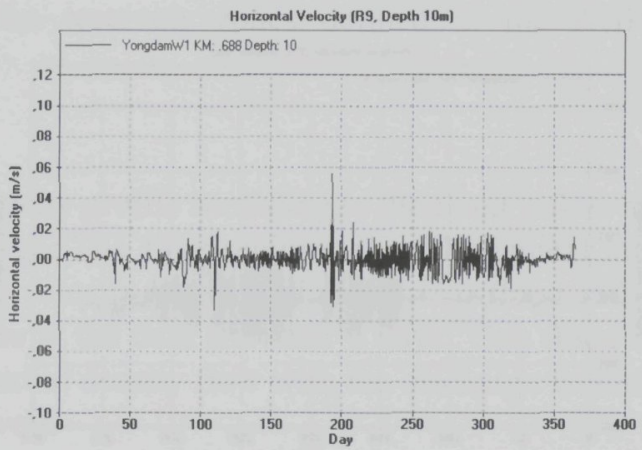

(b) Horizontal Velocity (R9, Depth: 10m)

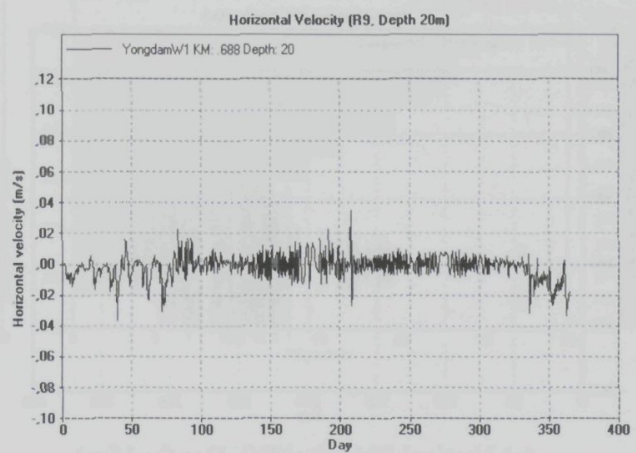

(d) Horizontal Velocity (R9, Depth: 20m)

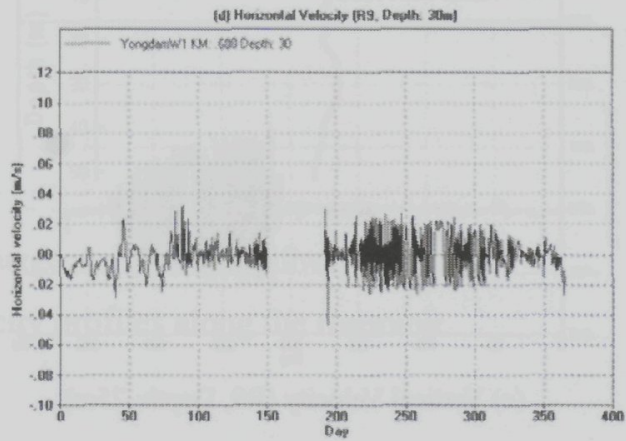

(f) Horizontal Velocity (R9, Depth: 30m)

Figure 7.14. Horizontal velocity variations at four depths $(5,10,15,20,25$ and 30 meters) of R9 in 2006. 
Figure 7.15 shows that the thermal stratification limits vertical mixing that could be generated by the horizontal flow. However, when the river interflow reaches R9 during the rainy season, vertical mixing develops at the intake. The spillway discharge allows the vertical flow to be damped at large depths (see 200 Julian days of Figure 7.15 (a) (e)).

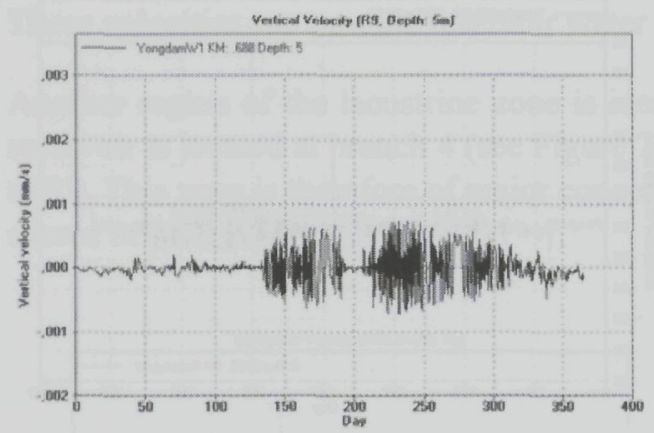

(a) Vertical Velocity (R9, Depth: 5m)

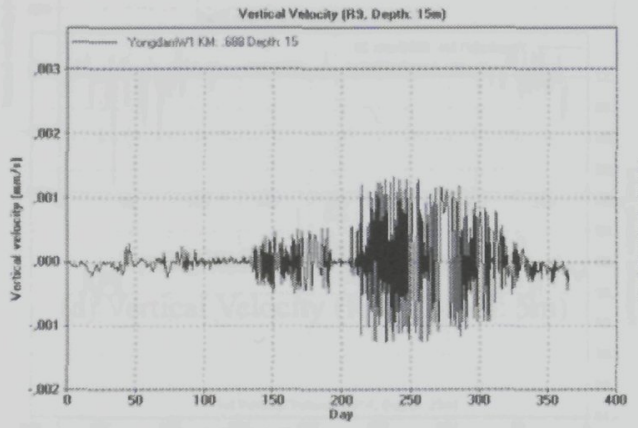

(c) Vertical Velocity (R9, Depth: $15 \mathrm{~m})$

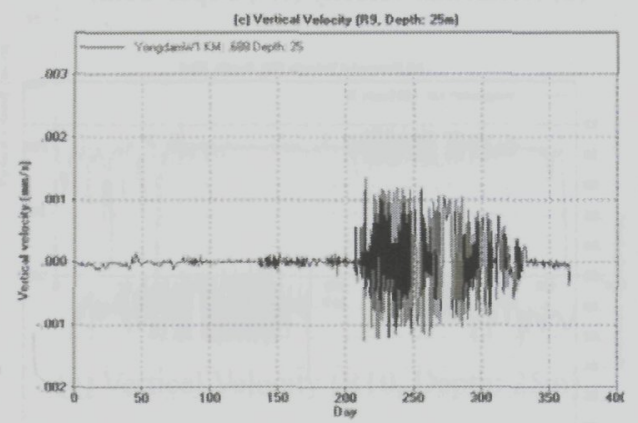

(e) Vertical Velocity (R9, Depth: 25m)

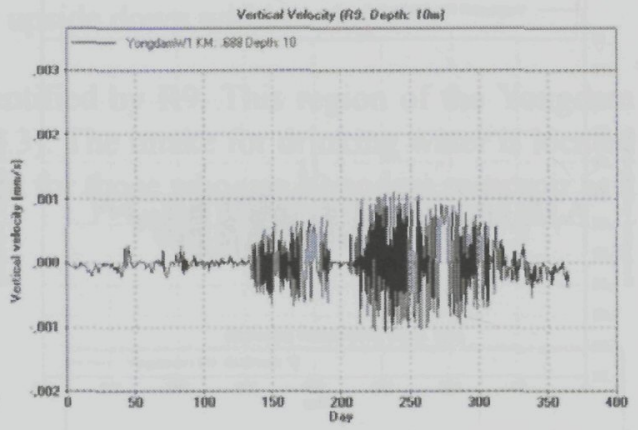

(b) Vertical Velocity (R9, Depth: 10m)

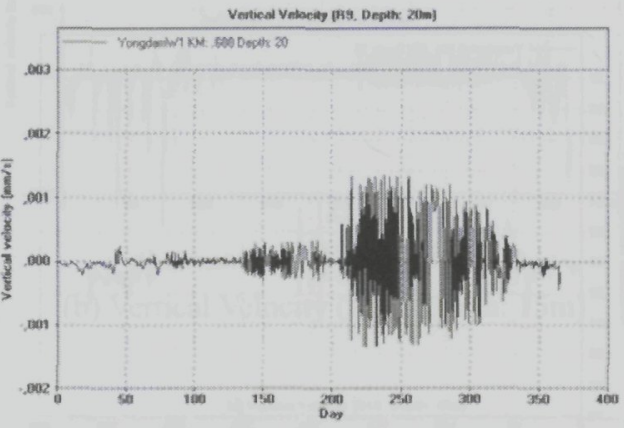

(d) Vertical Velocity (R9, Depth: 20m)

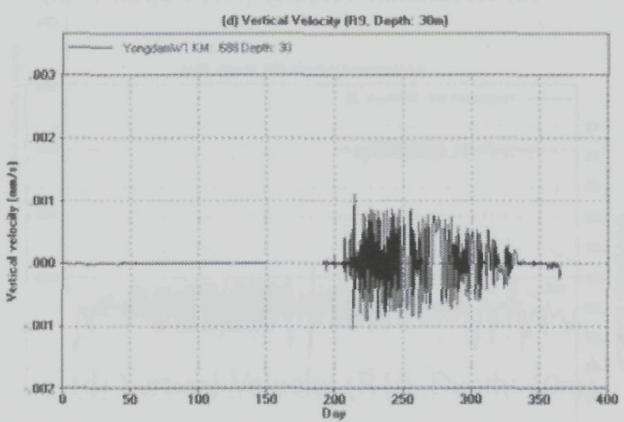

(f) Vertical Velocity (R9, Depth: 30m)

Figure 7.15. Vertical velocity variations at four depths $(5,10,15,20,25$ and 30 meters) of R9 in 2006.

The vertical oscillation at all depths of R9 may be due to a confluence mixing of the main stream and the branch 4 like R4. There is a horizontal backflow against the dam 
structure.

In summary, the horizontal and vertical velocities identify different regimes according to the three reservoir zones and three layers. The results of velocity variations show similar patterns to each other in the same reservoir zone, and therefore the results of the hydrodynamic modelling conform to the reservoir zones defined in the AGPT: lacustrine, transition and riverine zone.

\section{c) Profile of Horizontal Velocity}

Figure 7.16 shows the horizontal flow velocity profile according to the flood inflow and during the period of the spillway discharge. The flow regimes show horizontal local circulations. According to the horizontal velocity profile (Figure 7.16), flood inflow from the upstream of the reservoir reaches at the dam site after 6 days. This flood lag time makes it impossible to manage the turbid density flow with the discharge facilities located at lowest downstream of the reservoir.
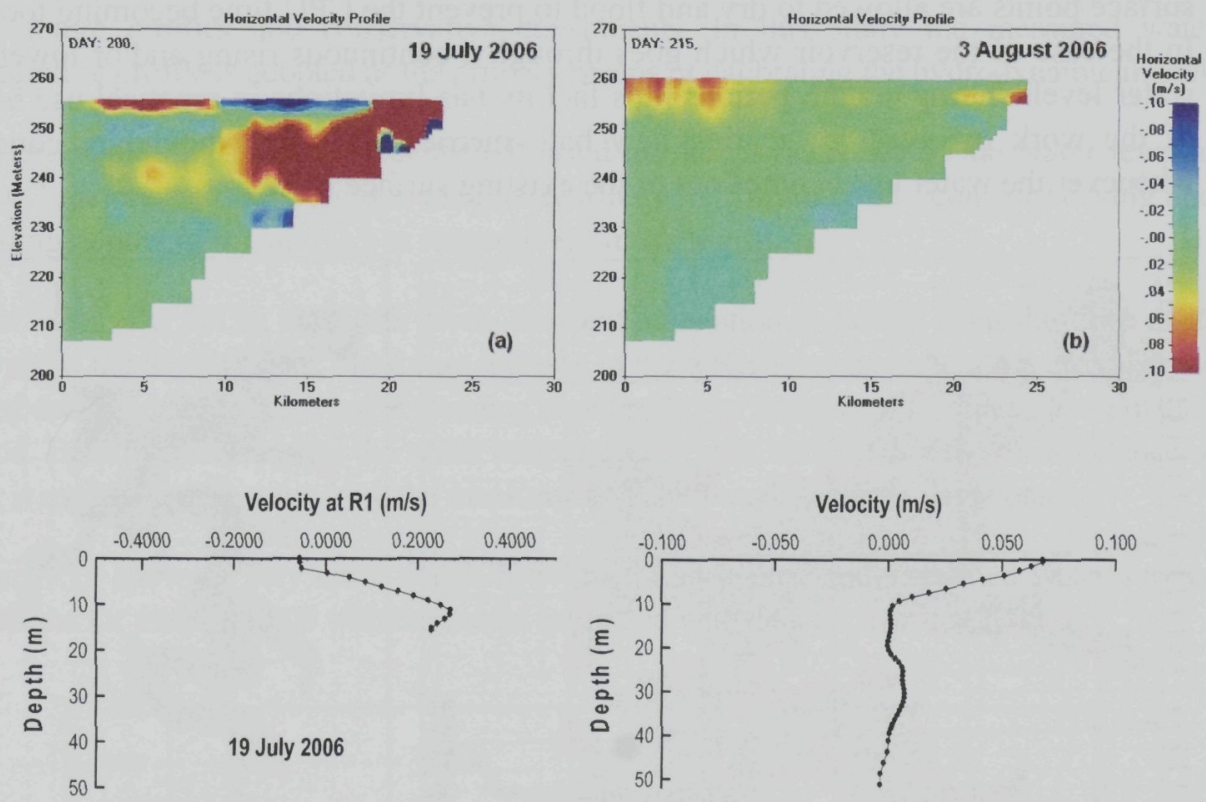

(a) Flood inflow without no spillway discharge

(b) with spillway discharge

Figure 7.16. Horizontal velocity profiles along the reservoir.

\section{2) MIKE3}

\section{a) Construction and Calibration}

In order to characterize fully variations in each of the horizontal, lateral and vertical directions, a full 3D model is required. However, long-term simulations with such a model are not practically feasible because of computation time constraints; therefore, 
simulations with the 3D model focus only on short-term critical periods when a density flow or advection and dispersion of constituents have been observed (on the order of one to two weeks). Even then there are still difficulties in setting up the initial conditions in the computational grid.

The hydrodynamic 3D model was constructed from the bathymetry using a staggered grid, and has a total of 42,544 computational points with a horizontal resolution of 90 meters and a vertical resolution of 2 meters (there are up to 33 layers depending on the deepest water depth of $66.2 \mathrm{~m}$ ). Time steps of 2.5 seconds and 15 seconds were adopted for calibration and validation respectively.

A very valuable facility in MIKE3 is its capability to include and exclude computational areas dynamically during the simulation or, in other words, to compute the flow in areas which sometimes dry out and sometimes are flooded (e.g. tidal flats). Continuity is fully preserved during the flooding and drying process as the water depths at the points which are dried out are saved and then reused when the point becomes flooded again. Only surface points are allowed to dry and flood to prevent the CPU time becoming too large. In the case of the reservoir which goes through a continuous rising and/or lowering in water levels during certain periods, this facility has limitations in practical use because of the work involved in creating new bathymetries and initial boundary conditions whenever the water level comes out of the existing surface layer.
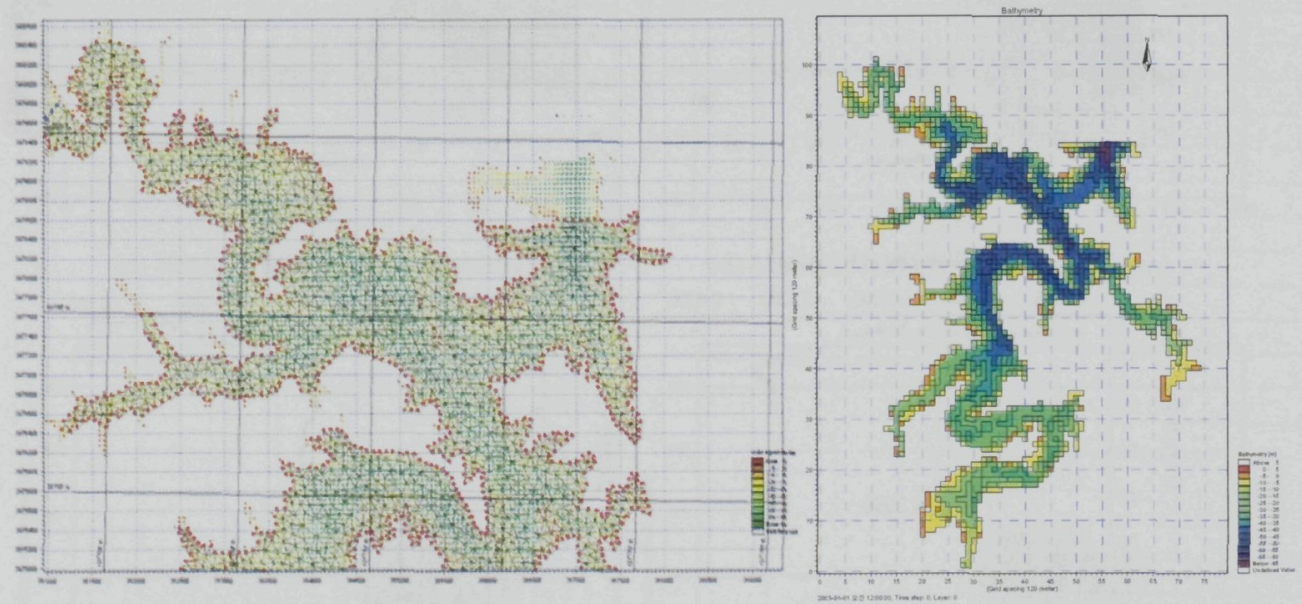

Figure 7.17. Bathymetry of Yongdam reservoir with mesh and grids in depth.

To avoid such intricate analysis, a flexible mesh using sigma coordinates was developed and applied (cf. MIKE 3 Flow Model-Flexible Mesh version and Cell-centered finite volume method). However, this form of discretisation creates its own problems such as a high vertical dispersion which is related to the vertical discretisation (sigma coordinates) as shown in Figure 7.18 (b). When the water level increases, the flexible grids play a role in creating high dispersion and mixing. 


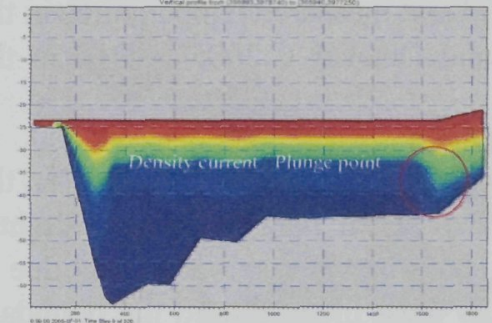

(a) Initial condition ( $1^{\text {st }}$ July 2005$)$

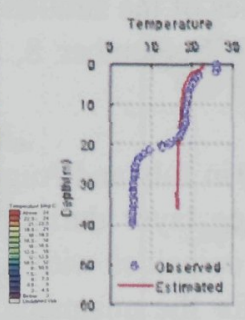

(b) After flood inflow ( $8^{\text {th }}$ July 2005)

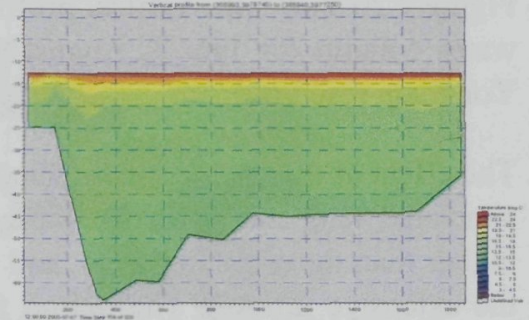

Figure 7.18. The results of MIKE3FM simulation in the strong thermal stratified Yongdam reservoir having storm water inflows.

Forcing functions for the hydrodynamic model include wind speed, reservoir outflow and inflow, precipitation, and heating and cooling at the air-water interface. A detailed calibration of the 3D model requires extensive measurements of 3D velocity, vertical temperature profiles and conservative constituents. In this study the measured water temperature profile is adopted as the primary means of calibrating the hydrodynamic model.

The key simulation period is identified based on the rise and fall of the water level at every 2 meters, and the period is readjusted after each simulation to consider drying and flooding depths of 0.1 and $0.3 \mathrm{~m}$ respectively in each grid.

In the case of a fall in the water level, the next simulation is started from the time of its arriving at the drying depth, but for an increase in the water level the time of its arrival at the top of each grid before arriving at flooding depth of $0.3 \mathrm{~m}$ is selected for the next simulation period. Before calibrating for the water temperature, it was verified that the mass balance of flow is maintained by comparing the measured and computed reservoir elevations.

Figure 7.19 shows a very close match between the measured and simulated water surface elevations for the year 2006 with the model calibrated with data for the year 2005 .

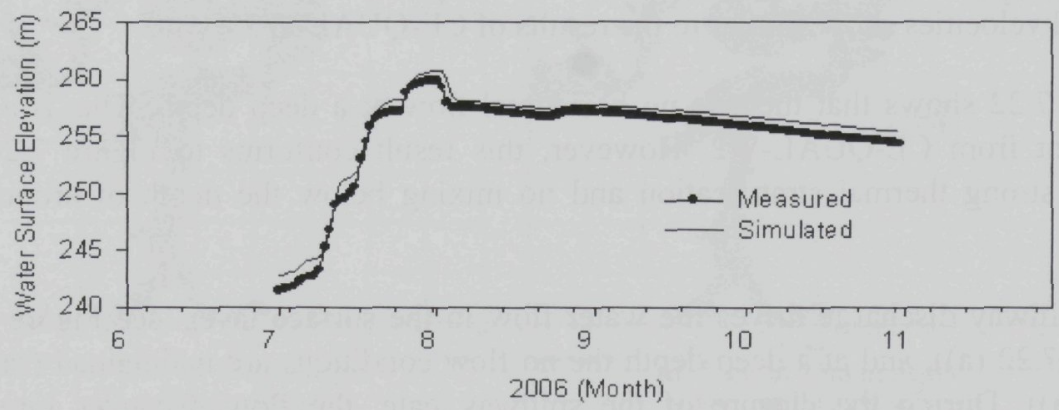

Figure 7.19. Modeled and Measured Water Surface Elevation in 2006. (Real line: Simulation data and dot: Measured data). 
Figure 7.20 shows the vertical profiles of the simulated and measured temperatures in the water column for July 7, August 9, September 8, and October 62005 at R10 in the Yongdam reservoir.

The effect of the double thermocline in July and August is reproduced well. However, the simulated temperature in the surface layer during September and October is slightly higher than the measured data, and coincides with a sharp increase in water level due to the extensive inflows. The reason for the mismatch is most likely due to the boundary conditions.

3D models require extensive and accurate data, and it is practically impossible to obtain meteorological, geological and weather data covering the whole of the reservoir surface. This is especially due to the fact that the discrepancy between measured and simulated water temperatures in the surface layer occurred at the same time as a sharp rise in water level, which generally generates much uncertainty and is the occasion of missing data. Actually in this period, it is impossible to access the sampling sites in the reservoir and the five tributaries because of flood flows and strong winds.

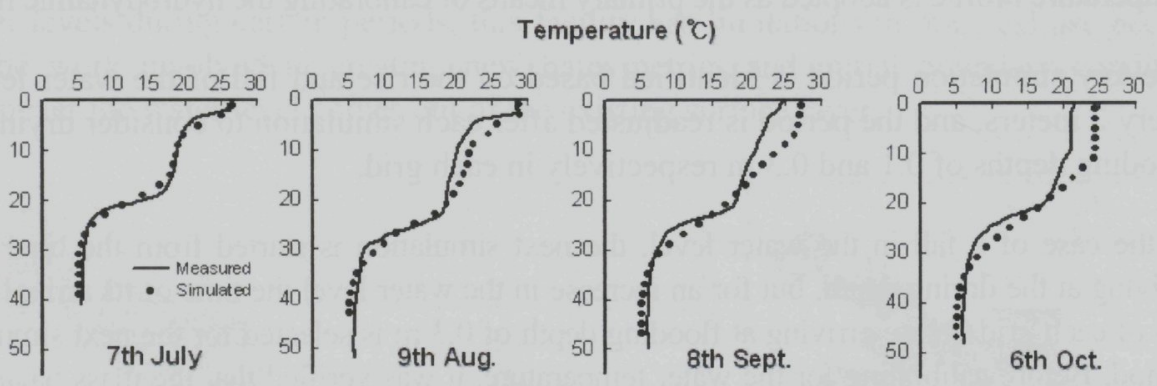

Figure 7. 20. Vertical Temperature distribution in MIKE 3 at R10 in 2006.

\section{b) Simulation Results for Three-Dimensional Velocity}

The simulation results of MIKE 3D is shown in Figure 7.21-23. The horizontal and vertical velocities show similar to the results of CE-QUAL-W2.

Figure 7.22 shows that there is no horizontal flow at a deep depth. This is a slightly different from CE-QUAL-W2. However, this result conforms to Figure 7.20 which shows strong thermal stratification and no mixing below the depth of around 15-20 meters.

The spillway discharge drives the water flow in the surface layer (see Figure 7.21 (a) and $\mathrm{c} ; 7.22(\mathrm{a})$ ), and at a deep depth the no flow conditions are maintained (see Figure 7.22 (a)). During the closure of the spillway gate, the flow disperses into several currents in the surface layer (see Figure 7.21 (b); 7.22 (b)). 


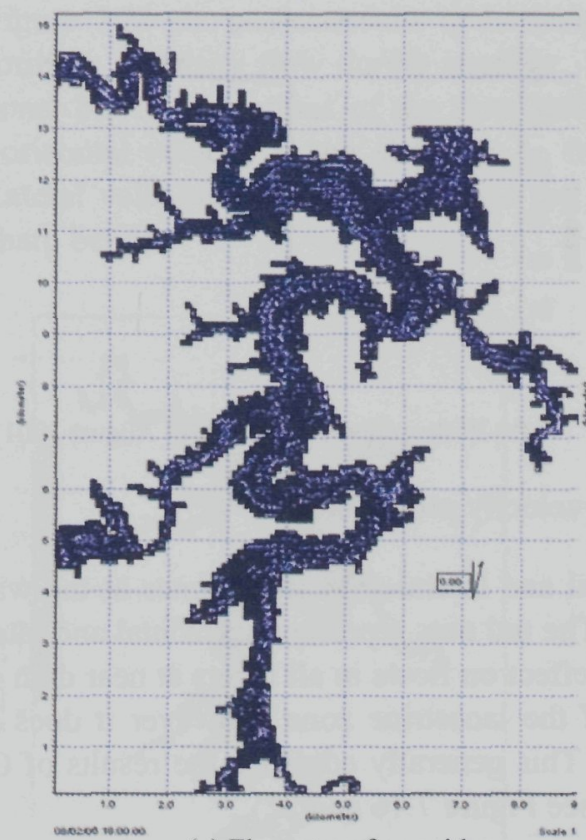

(a) Flow at surface with spillway discharge

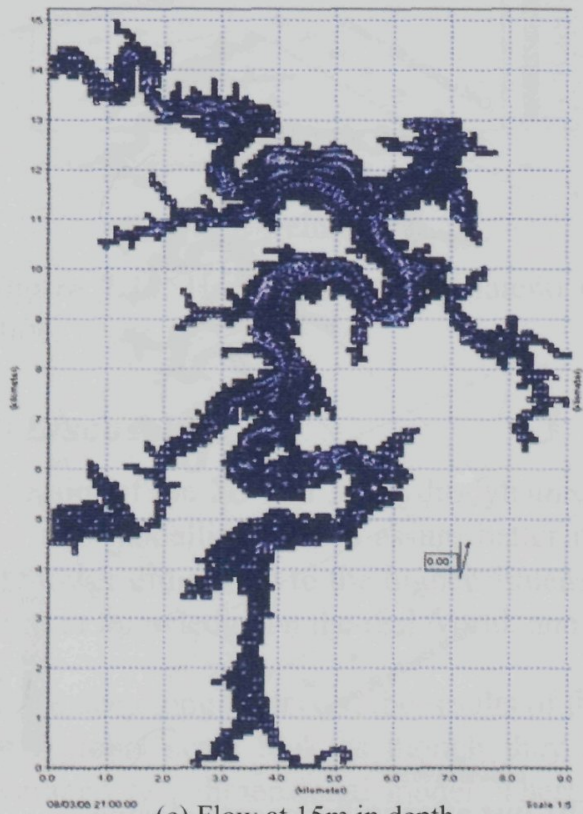

(c) Flow at $15 \mathrm{~m}$ in depth with spillway discharge

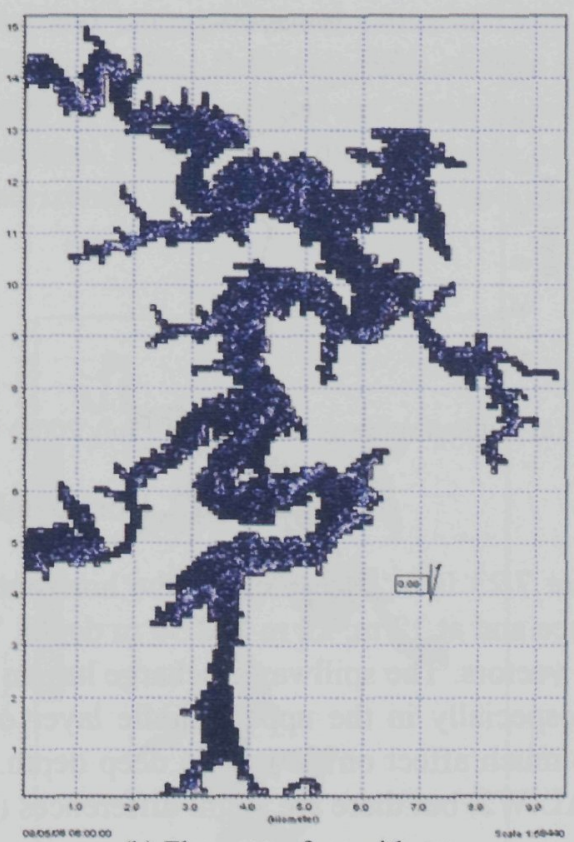

(b) Flow at surface without spillway discharge

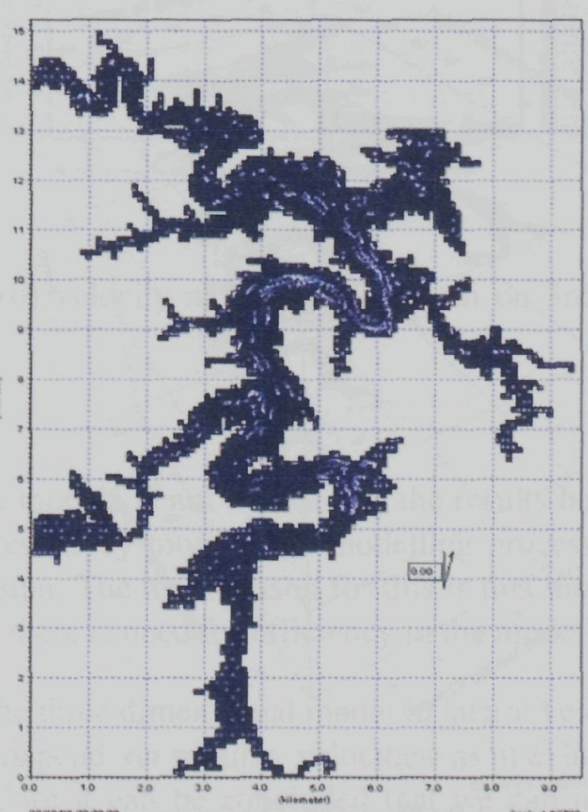

(d) Flow at $15 \mathrm{~m}$ in depth without spillway discharge

Figure 7.21. Flow visualization with and without spillway release at water surface and $15 \mathrm{~m}$ below water surface. 


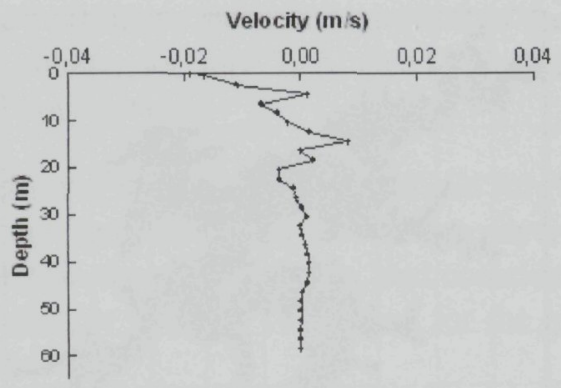

(a) Without spillway discharge (19 July 2006)

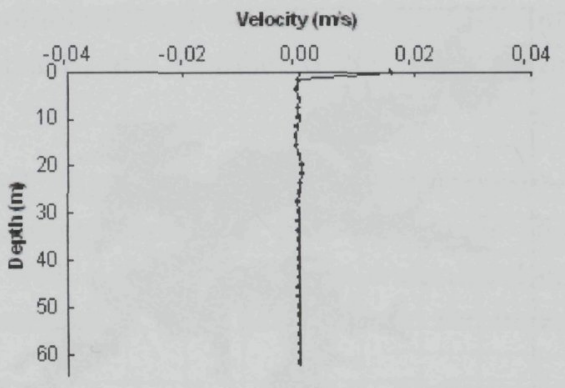

(b) With spillway discharge (3 August 2001)

Figure 7.22. Horizontal velocity profile at R10.

Figure 7.23 is a 'slice plot' of the horizontal and lateral velocity regimes at the water surface and at $12 \mathrm{~m}, 22 \mathrm{~m}$ and $36 \mathrm{~m}$ depth. The red dots represent horizontal and lateral flow vectors. The spillway discharge has an effect on flows in all layers at near dam site and especially in the upper middle layer of the lacustrine zone. However it does not have much affect on flows at a deep depth. This generally confirms the results of CEQUAL-W2, but there are slight differences (see Figure 7.16 and 22).

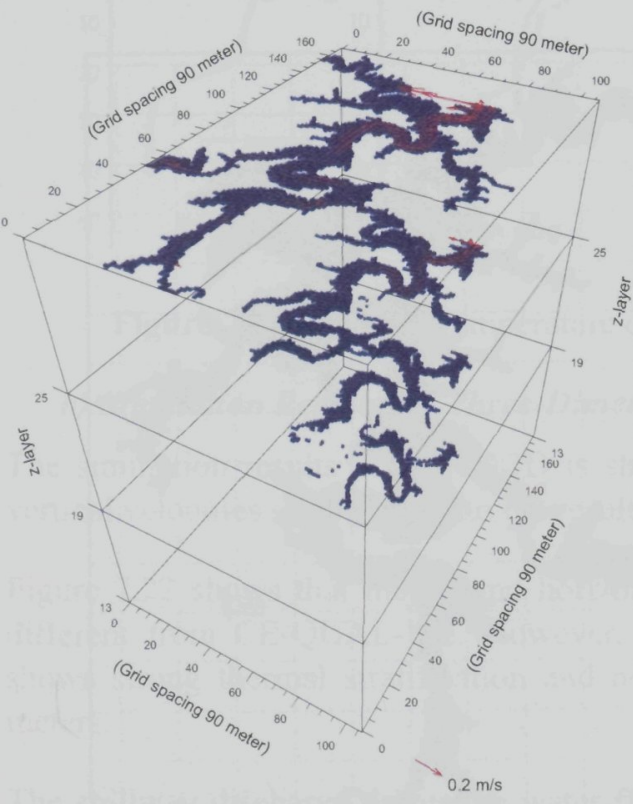

08/03/06 15:00:00

(a) Spillway discharge

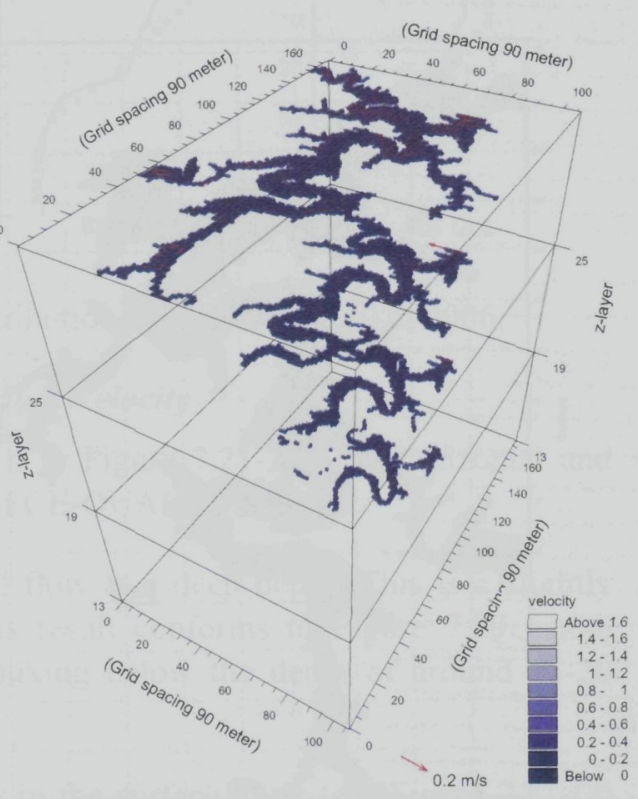

$08 / 04 / 06150000$

(b) Just after spillway gate closing

Figure 7.23. Slice plot of horizontal and lateral velocity vectors at water surface, 12,22 , and 36 meters below water surface. 
Figure 7.24 shows the results of horizontal and lateral flow velocities at $15 \mathrm{~m}$ depth forming a density flow during summer. The horizontal flow velocities change in each zone, but the variations of the flow velocity in lateral sections are small because the horizontal velocity is too slow due to frictional resistance in the grid scale of $90 \mathrm{~m}$. Lateral velocities are also similar to the horizontal velocities except in the location of sharp bends.

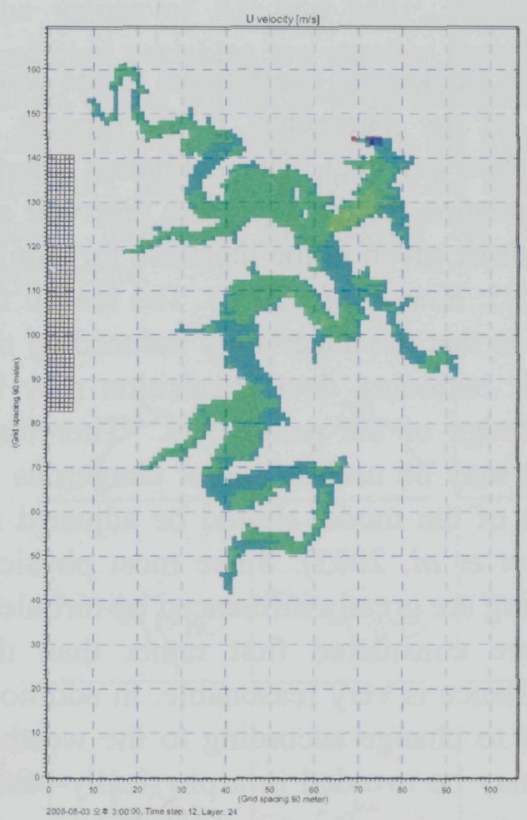

(a) U-velocity
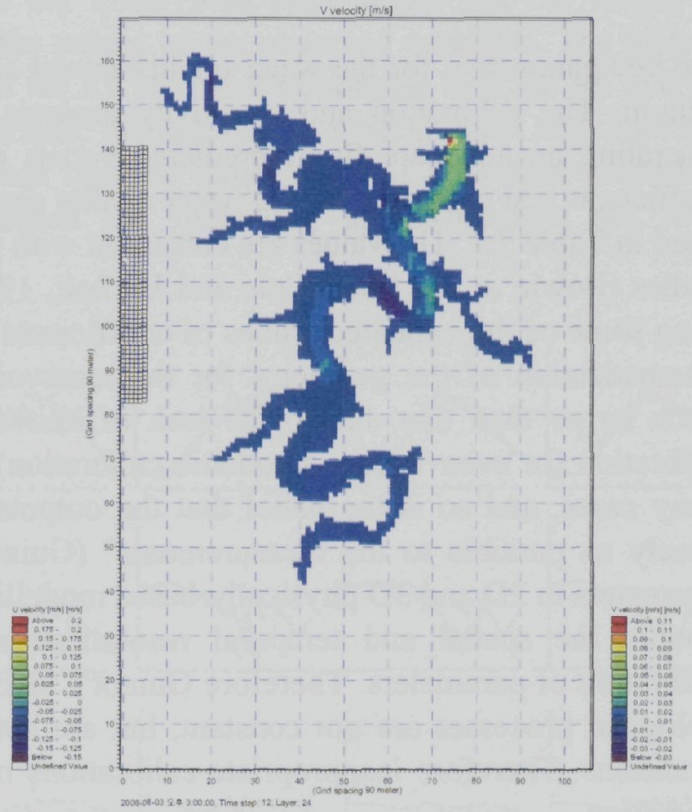

(b) V-velocity

Figure 7.24. Horizontal (a) and lateral (b) velocity at 15 meters' depth on 3rd Aug. 2006.

\section{3) Discussion}

The aim of the 2D and 3D hydrodynamic models is not to compare the results between the two modelling, but to assure better results by moving the modelling process from the lower dimension to the higher dimension. The main reason for this is that there is a limit of knowledge on the real world, and there is need for efficiency in the modelling.

In the deep long reservoir, the results of the three dimensional model in lateral velocities at a global scale look as though they depend on similar velocities as the laterally averaged two dimensional model. Therefore it can be concluded that we can use the two-dimensional model for water quality in a long, narrow, deep reservoir such as the Yongdam reservoir, even though there may be some difference at a smaller scale.

\subsubsection{Water Quality Modelling}


The hydrodynamic model component of CE-QUAL-W2, as used in this study, was validated against MIKE3. Subsequently, the water quality modelling was carried out using the water quality module of CE-QUAL-W2. The boundary conditions for the water quality model include dissolved oxygen, Chl-a, and nutrient concentrations at the ends of the main stem of the reservoir. In addition, concentrations of dissolved oxygen, Chl-a, and nutrients in the tributaries are also provided. Numerous model runs were conducted to calibrate the water quality model parameters.

The key parameters for the water quality model are the kinetic coefficients in the water column. The calibration and sensitivity analysis of the parameters is the process of generating an adequate fit of predicted versus observed data. The calibrated kinetic coefficients and constants for the water quality simulation for the Yongdam reservoir are listed in Table 7.1. The values are consistent with those given in the literature for similar studies (Bowie et al., 1985; Cole and Buchak, 1995; Kuo et al., 2006). The reason for using some of the validated values of coefficients is that the uncertainty induced by the schematization of the geometry for the reservoir including data acquisition may be much larger than that due to the lack of knowledge of the parameters. "Therefore, calibration (or even worse, automatic calibration) may be useless and/or dangerous in many cases, and so is the belief that the outputs of the model should be adjusted as closely as possible to the measurements" (Guinot et al, 2003). Since most physical parameters in 2D and 3D physically-based modelling are greatly influenced by turbulent motion, the spatial and temporal resolutions are considered first rather than the calibration of parameters. Therefore Guinot's insistence is very reasonable. In addition, biological processes are not constant, but are apt to change according to the weather conditions. Therefore inappropriate calibrations may be avoided in a physically-based modelling.

Table 7.1. The kinetic coefficients and values of CE-QUAL-W2 for the Yongdam reservoir.

\begin{tabular}{|c|c|c|c|}
\hline Eutrophication coefficients & Values & Eutrophication coefficients & Values \\
\hline Horizontal eddy viscosity $\left(\mathrm{m}^{2} / \mathrm{s}\right)$ & 1.000 & Light saturation intensity at maximum & 350.0 \\
\hline Horizontal eddy diffusivity $\left(\mathrm{m}^{2} / \mathrm{s}\right)$ & 1.000 & photosynthetic rate $\left(\mathrm{W} / \mathrm{m}^{2}\right)$ & \\
\hline $\begin{array}{l}\text { Light extinction coefficient for pure water } \\
\left(\mathrm{m}^{-1}\right)\end{array}$ & 0.450 & $\begin{array}{l}\text { Algal half-saturation for phosphorus } \\
\text { limited growth }(\mathrm{mg} / \mathrm{L})\end{array}$ & 0.005 \\
\hline Algal growth rate $\left(20^{\circ} \mathrm{C}\right)\left(\right.$ day $\left.^{-1}\right)$ & 1.600 & Algal half-saturation for nitrogen limited & 0.030 \\
\hline Algal respiration rate $\left(\right.$ day $\left.^{-1}\right)$ & 0.050 & growth $(\mathrm{mg} / \mathrm{L})$ & \\
\hline Algal excretion rate $\left(\right.$ day $\left.^{-1}\right)$ & 0.040 & Ammonia nitrification rate $\left(\right.$ day $\left.^{-1}\right)$ & 0.025 \\
\hline Algal mortality rate $\left(\right.$ day $\left.^{-1}\right)$ & 0.030 & Nitrate denitrification rate $\left(\right.$ day $\left.^{-1}\right)$ & 0.150 \\
\hline Algal settling rate (m/day) & 0.100 & Sediment oxygen demand $\left(\mathrm{g} / \mathrm{m}^{2} /\right.$ day $)$ & 1.000 \\
\hline
\end{tabular}

The water quality and the temperature results compared with measured data in the surface layer at all sampling stations are shown in Figure 7.25. The Chl-a, total phosphorus, total nitrite concentrations, concentrations of dissolved oxygen, and the biochemical oxygen demand (BOD) measured in the field are matched well at all stations by the model results. A peak of Chl-a is calculated by the model in the summer, followed by a sharp 
decline in the rest of the year. Dissolved oxygen (DO) levels in the surface depend strongly on the water temperature.

A qualitative evaluation of the model verification can be made by 'goodness of fit' statistics. Table 7.2 shows the results of 'goodness of fit' statistics (ref. Chapter 4.6.2) for algal concentrations in the surface layer during 2006.

The values of $\mathrm{AE}$ and $\mathrm{MEF}$, which are lower than zero, mean that the values of predicted variables are smaller than the values of the observed variables. The general values of the goodness-of-fit statistics for R9 are more reasonable than for R6 and R7 considering the uncertainty in the hydraulic mixing, temperature gradient and unknown inhibitors for algal growth. This means that the target point of numerical modelling should focus on the region for which the modelling approximations are most appropriate.
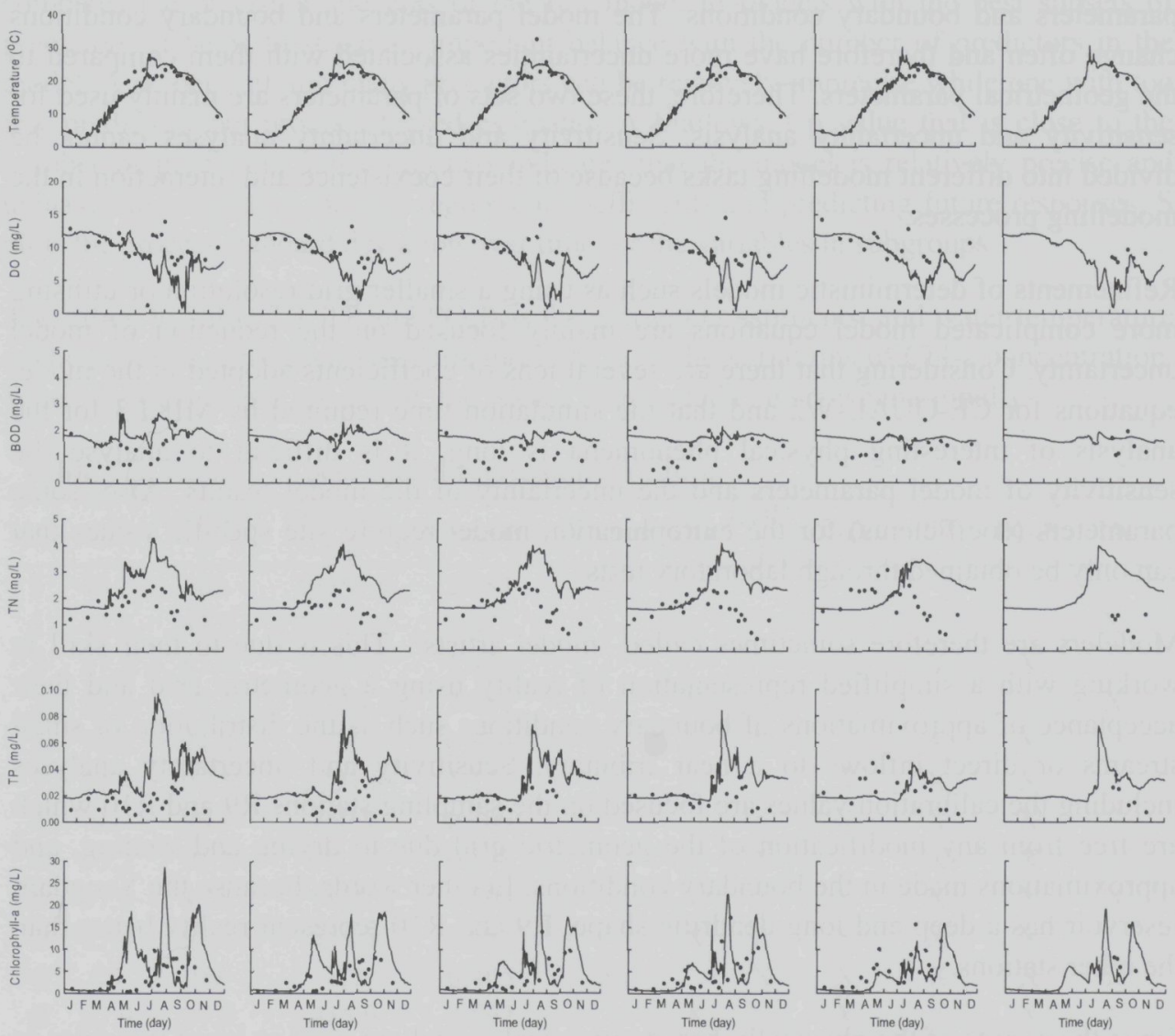

(a) R2

(b) R4

(c) R6

(d) R7

(e) R9

(f) R10

Figure 7.25. Modeled and Measured water qualities in the surface layer at four measuring points in 2006 (Real line: Simulation data and dot: Measured data). 
Table 7.2. Goodness-of-Fit Statistics between predictions and observations at R10 in 2006.

\begin{tabular}{ccccccc}
\hline $\begin{array}{c}\text { Sampling } \\
\text { station No. }\end{array}$ & $\begin{array}{c}\text { R } \\
\text { (correlation } \\
\text { coefficient) }\end{array}$ & $\begin{array}{c}\text { RMSE } \\
\text { (root mean } \\
\text { squared error) }\end{array}$ & $\begin{array}{c}\text { RI } \\
\text { (reliability } \\
\text { index) }\end{array}$ & $\begin{array}{c}\text { AE } \\
\text { (average } \\
\text { error) }\end{array}$ & $\begin{array}{c}\text { AAE } \\
\text { (average } \\
\text { absolute } \\
\text { error) }\end{array}$ & $\begin{array}{c}\text { MEF } \\
\text { (modeling } \\
\text { efficiency) }\end{array}$ \\
\hline 6 & 0.08 & 4.81 & 1.85 & -0.08 & 4.67 & -2.56 \\
7 & 0.39 & 3.52 & 1.70 & 1.15 & 3.57 & -0.63 \\
9 & $\mathbf{0 . 6 2}$ & $\mathbf{2 . 7 7}$ & $\mathbf{1 . 6 2}$ & $\mathbf{0 . 7 4}$ & $\mathbf{2 . 6 8}$ & $\mathbf{0 . 1 2}$ \\
10 & 0.47 & 4.23 & 1.75 & 1.06 & 3.81 & -0.17 \\
\hline
\end{tabular}

\subsubsection{Sensitivity and Uncertainty}

In the model system there are many parameters that influence the results of a model. These parameters can be divided into three categories: geometrical and model parameters and boundary conditions. The model parameters and boundary conditions change often and therefore have more uncertainties associated with them compared to the geometrical parameters. Therefore, these two sets of parameters are mainly used for sensitivity and uncertainty analysis. Sensitivity and uncertainty analyses cannot be divided into different modelling tasks because of their coexistence and interaction in the modelling processes.

Refinements of deterministic models such as using a smaller grid resolution or utilising more complicated model equations are mainly focused on the reduction of model uncertainty. Considering that there are several tens of coefficients adopted in the model equations for CE-QUAL-W2 and that the simulation time required by MIKE3 for the analysis of interesting physical phenomena is long, it is difficult to analyse the sensitivity of model parameters and the uncertainty of the model results. Also, some parameters (coefficients) for the eutrophication model require site specific values that can only be obtained through laboratory tests.

Modelers are therefore sometimes called 'model artists'. This is due to their skill in working with a simplified representation of reality using a geometric grid and their acceptance of approximations of boundary conditions such as the distribution of small streams or direct inflows to a near tributary. Sensitivity and uncertainty analyses including the calibration values are focused on the sampling stations R9 and R10 which are free from any modification of the geometric grid due to drying and wetting, and approximations made in the boundary conditions. In other words, because the Yongdam reservoir has a deep and long dendritic shape, $\mathrm{R} 9$ and $\mathrm{R} 10$ represent reality better than the other stations.

Since the results of the physically-based numerical model are used as input variables to the data-driven model as shown Figure 7.1, the most sensitive water quality variables for algae growth (Chl-a concentration) and the uncertainty of the predicted Chl-a concentrations are analysed as follows. 


\section{1) Sensitivity}

The main active constituents for computation by the CE-QUAL-W2 model in this study are water temperature (W.temp.), dissolved oxygen (DO), BOD5, COD $\mathrm{Cr}_{\text {, total }}$ phosphorus(TP), inorganic phosphate $\left(\mathrm{PO}_{4} \mathrm{P}\right)$, total nitrogen (TN). The sensitivity analysis was carried out on water quality constituents instead of parameters in the governing equations. The results were analysed using Mintab V.15, which identified the best subset regression for chlorophyll-a in Table 7.3.

A good model should have a high coefficient of determination, $r^{2}$, and adjusted $r^{2}$, a small standard deviation, S, and a Mallows' Cp close to the number of predictors in the model and a constant. The adjusted $r^{2}$ is a useful tool for comparing the explanatory power of models with different numbers of predictors. The adjusted $r^{2}$ will increase only if the new term improves the model more than would be expected by chance. It will decrease when a predictor improves the model less than expected by chance. Mallows' $\mathrm{Cp}$ compares the precision and bias of the full model to models with the best subsets of predictors. It helps to strike an important balance with the number of predictors in the model. A model with too many predictors can be relatively imprecise while one with too few predictors can produce biased estimates. A Mallows' $\mathrm{Cp}$ value that is close to the number of predictors and a constant indicates that the model is relatively precise and unbiased in estimating the true regression coefficients and predicting future responses. $\mathrm{S}$ plots the process standard deviation over time for the variables in subgroups.

According to Table 7.3, TP and $\mathrm{PO}_{4} \mathrm{P}$ as the nutrient constituents, and water temperature as the physical constituent, explain about $90 \%$ of the variations of Chl-a concentration. When the other items are added, the values of adjusted $r^{2}$ increase marginally.

Table 7.3. Results of best subset regression on Chl-a.

\begin{tabular}{cccccccccccc}
\hline Vars & $\mathrm{r}^{2}$ & $\begin{array}{c}\text { Adjusted } \\
\mathrm{r}^{2}\end{array}$ & $\begin{array}{c}\text { Mallows' } \\
\mathrm{C}\end{array}$ & $\mathrm{S}\left(\mathrm{mg} / \mathrm{m}^{3}\right)$ & W.temp & $\mathrm{DO}$ & $\begin{array}{c}\mathrm{BOD} \\
5\end{array} \mathrm{COD}_{\mathrm{Cr}}$ & $\mathrm{TP}$ & $\mathrm{PO}_{4} \mathrm{P}$ & $\mathrm{TN}$ \\
\hline 1 & 46.2 & 46 & 19357.8 & 2.983 & $\mathrm{X}$ & & & & & & \\
1 & 42.8 & 42.7 & 20571.2 & 3.073 & & & & & & & $\mathrm{X}$ \\
2 & 87.5 & 87.4 & 4231.7 & 1.441 & & & & & $\mathrm{X}$ & $\mathrm{X}$ & \\
2 & 57.6 & 57.4 & 15174.1 & 2.651 & & $\mathrm{X}$ & & & & & $\mathrm{X}$ \\
3 & 94.8 & 94.8 & 1531.3 & 0.926 & & & $\mathrm{X}$ & & $\mathrm{X}$ & $\mathrm{X}$ & \\
3 & 91.4 & 91.3 & 2791.8 & 1.195 & & & & $\mathrm{X}$ & $\mathrm{X}$ & $\mathrm{X}$ & \\
4 & 97 & 96.9 & 750.9 & 0.709 & & & $\mathrm{X}$ & $\mathrm{X}$ & $\mathrm{X}$ & $\mathrm{X}$ & \\
4 & 95.2 & 95.1 & 1413.3 & 0.897 & & $\mathrm{X}$ & $\mathrm{X}$ & & $\mathrm{X}$ & $\mathrm{X}$ & \\
5 & 97.9 & 97.8 & 434 & 0.599 & & & $\mathrm{X}$ & $\mathrm{X}$ & $\mathrm{X}$ & $\mathrm{X}$ & $\mathrm{X}$ \\
5 & 97.4 & 97.4 & 581.5 & 0.653 & & $\mathrm{X}$ & $\mathrm{X}$ & $\mathrm{X}$ & $\mathrm{X}$ & $\mathrm{X}$ & \\
6 & 99 & 99 & 8.3 & 0.405 & $\mathrm{X}$ & & $\mathrm{X}$ & $\mathrm{X}$ & $\mathrm{X}$ & $\mathrm{X}$ & $\mathrm{X}$ \\
6 & 97.9 & 97.9 & 415.9 & 0.592 & & $\mathrm{X}$ & $\mathrm{X}$ & $\mathrm{X}$ & $\mathrm{X}$ & $\mathrm{X}$ & $\mathrm{X}$ \\
7 & 99 & 99 & 8 & 0.405 & $\mathrm{X}$ & $\mathrm{X}$ & $\mathrm{X}$ & $\mathrm{X}$ & $\mathrm{X}$ & $\mathrm{X}$ & $\mathrm{X}$ \\
6 & 97.9 & 97.9 & 415.9 & 0.592 & & $\mathrm{X}$ & $\mathrm{X}$ & $\mathrm{X}$ & $\mathrm{X}$ & $\mathrm{X}$ & $\mathrm{X}$ \\
7 & 99 & 99 & 8 & 0.405 & $\mathrm{X}$ & $\mathrm{X}$ & $\mathrm{X}$ & $\mathrm{X}$ & $\mathrm{X}$ & $\mathrm{X}$ & $\mathrm{X}$ \\
\hline
\end{tabular}


Other constituents, with the exception of nitrogen, are products of algal growth and decay, and do not directly influence algal growth. This may be due to simultaneous phenomena taking place with algal photosynthesis (production of biomass) and respiration (consumption of biomass). Therefore the best subset regression is done with these three constituents and Chl-a concentration to explore how and to what extent these parameters contribute to variations in the Chl-a concentration.

The results of Table 7.4 show that the three constituents can give about $90 \%$ of $\mathrm{r}^{2}$ in total variation level for predicting $\mathrm{Chl}$-a concentration. The coefficients related to phosphorus concentration and algal growth and decay are the most sensitive and important parameters to predict and control eutrophication.

Table 7.4. Results of best subset regression on Chl-a.

\begin{tabular}{cccccccc}
\hline Vars. & $\mathrm{r}^{2}$ & Adjusted $\mathrm{r}^{2}$ & Mallows' $\mathrm{Cp}$ & $\mathrm{S}\left(\mathrm{mg} / \mathrm{m}^{3}\right)$ & W.temp & $\mathrm{T}-\mathrm{P}$ & $\mathrm{PO}_{4} \mathrm{P}$ \\
\hline 1 & 46.2 & 46 & 1671.9 & 2.983 & $\mathrm{X}$ & & \\
1 & 24.1 & 23.9 & 2503.12 & 3.540 & & $\mathrm{X}$ & \\
2 & 87.5 & 87.4 & 114.3 & 1.441 & & $\mathrm{X}$ & $\mathrm{X}$ \\
2 & 48.3 & 48 & 1592.81 & 2.926 & $\mathrm{X}$ & $\mathrm{X}$ & \\
3 & 90.4 & 90.4 & 4 & 1.260 & $\mathrm{X}$ & $\mathrm{X}$ & $\mathrm{X}$ \\
\hline
\end{tabular}

\section{2) Uncertainty}

There are two kinds of uncertainty in the modeling process: temporal and/or spatial variations in a whole water body, and the uncertainty in the predictions. The uncertainty in the physically-based deterministic models is confined primarily to the imprecise predictions assuming the boundary conditions are credible.

Although the water quality models predict a single value for a constituent at a point in time and space, the relationships between the pollutant loading and the response to the flow of the water can never be perfectly expressed by a model. Regardless of the accuracy and complexity of the physical, chemical, and biological processes as modeled, there will be a residual uncertainty due to the natural variability, mis-specification of the boundary conditions and measurement errors. Thus, the approaches to sensitivity, variability and uncertainty adopt particular seasons for statistical analysis.

The uncertainty in the physically-based modelling is focused on the response variable which is important to the modeler. However there are many uncertain factors such as numerous model coefficients and boundary conditions etc. The test for normality of the data was executed on Minitab V.15. Even though the 23 measurement instances in the Yongdam reservoir during 2003 is rather small for uncertainty analysis, the measured data of algae concentration has a normal distribution because the p-value is 0.053 , which is greater than 0.05 , the confidence level of the test for normality.

The most critical uncertainty is in the input data for water quality. In fact we use at best biweekly data for the input of water quality for the physically-based models. There can 
be a strong possibility of error between the model input for water quality and the real data due to the lack of measurements during the flood season.

Since the p-value for the results of the CE-QUAL-W2 simulation is about 0.01 , there are many uncertainties in the modelling processes. However we cannot say, at present, that the constructed model never represents the reservoir phenomena because the results of the water quality model calculate average values in the discretised domain.

\subsubsection{Quality Assurance}

So far as quality assurance is concerned, firstly mistakes were minimized as far as possible during the modelling process by following an agreed procedure and the predicted values were fitted closely to the measured values. Stream flows from small and un-gauged tributaries were calculated based on their watershed areas. According to the hydrodynamic model validation analysis, the time-variable water surface elevation data were used to estimate the reservoir outflow through a water mass balance.

The kinetic coefficients and constants for the water quality simulation were kept as much as possible to their default values to reduce another uncertainty introduced by their adjustments. The MIKE3 hydrodynamic model confirmed the 2D hydrodynamic model of CE-QUAL-W2. Water temperature and DO show good agreement. In that algal growth is known to be influenced by many functions, the results of this study are acceptable. $90 \%$ of the simulated values are distributed in the $90 \%$ confidence interval of the measured data; see Figure 7.26.

Probability Plot of Chl-a at Sta. No.10

Normal - $95 \%$ CI

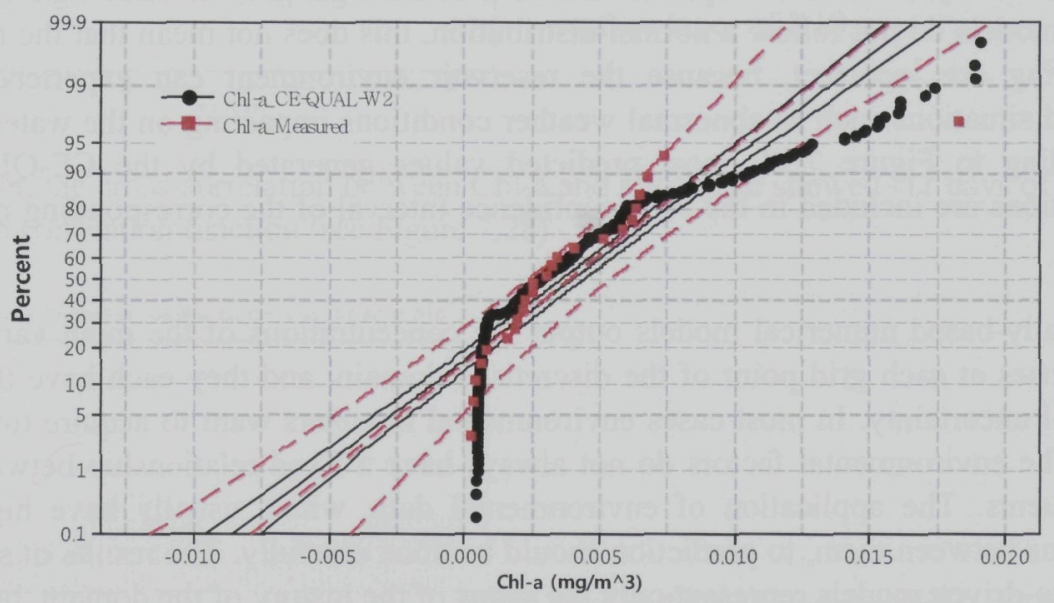

Figure 7.26. Probability intervals of simulated data and measured data on Chl-a concentration at R10. 
However, the hybrid model mentioned in Section7.1 was implemented for a further improvement in the quality of the results for the physically-based modelling of Chl-a concentration, which is described in Section 7.4 below.

\subsubsection{Discussion and Time Series Analysis}

\section{1) Discussion}

The 2D and 3D hydrodynamic models, CE-QUAL-W2 and MIKE3 were successfully constructed and verified during this study. However, the 3D hydrodynamic model using a flexible mesh (sigma-coordinate) needs further improvement for modelling a thermally stratified reservoir. The simulation time for water quality modelling using a $3 \mathrm{D}$ model is a limit. For the water quality modelling requires many times of model running due to changing parameters for a calibration. This is the reason that the water quality modelling using the 3D model was not carried out in this study nevertheless of its better results in the hydrodynamic modelling than the $2 \mathrm{D}$ model.

The Yongdam reservoir exhibits a pronounced vertical thermal gradient in the summer and undergoes significant overturning in the spring and late autumn. The flow regimes reveal horizontal and vertical local circulations. Global mixing occurs throughout the reservoir during the summer, starting at the tributary inflows. This is due to the large inflows with a lower temperature than the surface water of the reservoir. Inflows tend to intrude into the reservoir horizontally along the thermocline at the relevant temperature for the inflow water. Vertical mixing generated by the consequent shear stresses induces a local circulation along the reservoir.

These phenomena make it difficult to predict water quality variations in the Yongdam reservoir. They often induce spatial variations in the algal growth. Although the results of the models do not follow a normal distribution, this does not mean that the results of modelling are incorrect, because the reservoir environment can experience many unusual situations such as abnormal weather conditions impacting on the water quality. According to Figure 7.23 , most predicted values generated by the CE-QUAL-W2 simulations are included in the $95 \%$ confidence interval of the corresponding measured data.

Physically-based numerical models output the concentrations of the input variables as time series at each grid point of the discretised domain, and they each have their own residual uncertainty. In most cases environmental modelers want to acquire time series data. The environmental factors do not always have a fixed relationship between their constituents. The application of environmental data, which usually have high order reactions between them, to prediction should be done carefully. The results of statistical and data-driven models represent only the status of the history of the domain, but do not give any information about the future. The reason is that statistical and data-driven models do not reflect the physical, chemical and biological processes arising due to the many forcing functions such as weather condition. Therefore the environment should 
firstly be assessed by the physically-based model ahead of any statistical and datadriven modelling in case there have been significant changes in the domain such as pollutant reduction projects. Such projects create a new environment which is different to that in the past. In other words, the nutrient concentrations at sampling station R10 are not necessarily directly dependent on changes in the nutrient concentrations of the inflow water from the tributaries (from Sampling station T1 to T5).

Finally, to reduce global uncertainty, the modelling strategy is to focus on a few water quality constituents. If we try to fit all constituents to the measured data, much more uncertainty will be generated and then make the situation more complex to analyse.

\section{2) Time Series Analysis}

The time series data generated by the physically-based models give decision makers useful information as to the nature of the periodic cycles in the flow and pollutant variables, as well as an assessment of remedial action plans for the polluted reservoir. In this study, a recurrence cycle of algal blooms was estimated through auto correlation analysis and cross correlation between water quality parameters and data from the sampling stations. The recurrence cycle of algal blooms is about 15 days with $5 \%$ significance limits for the autocorrelations (see Figure 7.27). This number of days is close to that achieved in tests of the general algal growth rate (Tilman, 1982).

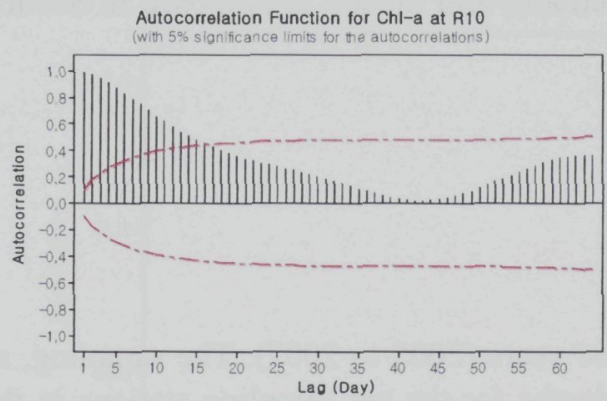

Figure 7.27. Autocorrelation of Chl-a at surface layer of R10.

However, the cross-correlation between Chl-a and phosphate showed -11 days of time lag with 0.5 correlation function (see Figure 7.28).

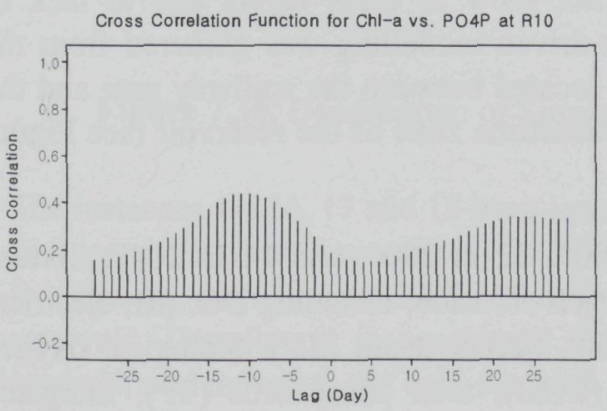

Figure 7.28. Cross-correlation of Chl-a vs. $\mathrm{PO}_{4} \mathrm{P}$ at surface layer of R10. 
The cross-correlations between Chl-a and other water quality parameters do not have any time lag or very low correlations. Therefore it is meaningless to analyse a spatial relationship between Chl-a and nutrients for the Yongdam reservoir. During the summer season, the relatively high concentrations of phosphate in the middle layer are the source of nutrients for algal growth in the surface layer. This implies that there is no time lag between $\mathrm{Chl}-\mathrm{a}$ in the surface layer and $\mathrm{PO}_{4} \mathrm{P}$ in the middle layer at R10; see Figure 7.29.

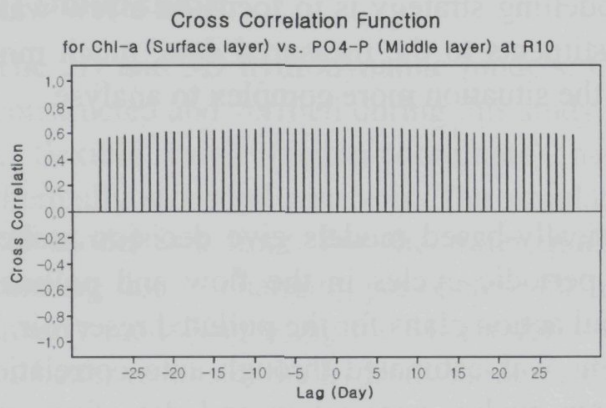

Figure 7.29. Cross-correlation of Chl-a (surface layer) vs. $\mathrm{PO}_{4} \mathrm{P}$ (middle layer) of R10.

The relatively accurate data generated by CE-QUAL-W2 for water temperature, phosphate and phosphorus, can be used in the prediction of Chl-a concentration for comparison with measured $\mathrm{Chl}$-a data through data-driven modelling. The methodology of combining two models (into what is termed a hybrid model) is proposed in Section 7.4. below.

\subsection{Data-Driven Modelling}

\subsubsection{Construction of Database}

During this study, data was collected for three years (2005 to 2007). The sampling, as mentioned in Chapter 6, was done at three depths for the ten sampling stations in the reservoir. Since all sampling stations have similar patterns regarding variations in water quality, sampling station R9 was selected for detailed study because of its proximity to the water intake for supply purposes. Data from the middle and bottom layers were not included in the analysis because algae do not grow in these layers due to lack of sunlight. Therefore the data set for the data-driven modelling was gathered from the surface layer of sampling station R9. This is located between the spillway gate and the intake gate for drinking water supply in the lacustrine zone of the reservoir (see Figure $6.4)$.

The total number of samples is 60: 23 in 2005, 23 in 2006, and 14 in 2007. Sixteen attributes of water quality were analyzed on each occasion, including $\mathrm{DO}, \mathrm{pH}$, electrical conductivity (EC), water temperature, turbidity, Secchi depth (SD), biological oxygen demand (BOD), chemical oxygen demand (COD), total phosphorus (TP), inorganic phosphate $\left(\mathrm{PO}_{4}-\mathrm{P}\right)$, total nitrogen $(\mathrm{TN})$, ammonia nitrogen $\left(\mathrm{NH}_{4}-\mathrm{N}\right.$ or $\left.\mathrm{NH}_{4}{ }^{+} \mathrm{N}\right)$, nitrate 
$\left(\mathrm{NO}_{3}{ }^{-}\right)$, nitrite $\left(\mathrm{NO}_{2}{ }^{-}\right)$, dissolved organic carbon (DOC) and chlorophyll-a (Chl-a).

\subsubsection{Analysis of Data}

In multivariate regression using machine learning techniques, the conditions for a suitable regression are a highly correlated data set and a limited number of variables. There needs to be a degree of similarity between the instances for each variable to find the necessary relationships, but this is not always so in the natural environment.

The attributes (variables) of water quality used for the analysis have been restricted to four items: water temperature, total phosphorus (TP), inorganic (ortho-) phosphate $\left(\mathrm{PO}_{4}-\mathrm{P}\right)$ and $\mathrm{Chl}-\mathrm{a}$ concentration; see Section 7.2. The reason for restricting the analysis to these four items is that together they explain more than $90 \%$ of Chl-a variance in the Yongdam reservoir. It is also known that the application of more input variables would make the model inefficient (Jørgensen, 1997).

The data set from 2005 to 2007 was tested to discover similarities between instances. Figure 7.30 shows the result of identifying single linkage similarities, which are based on the distances between vectors with the four variables for each instance.

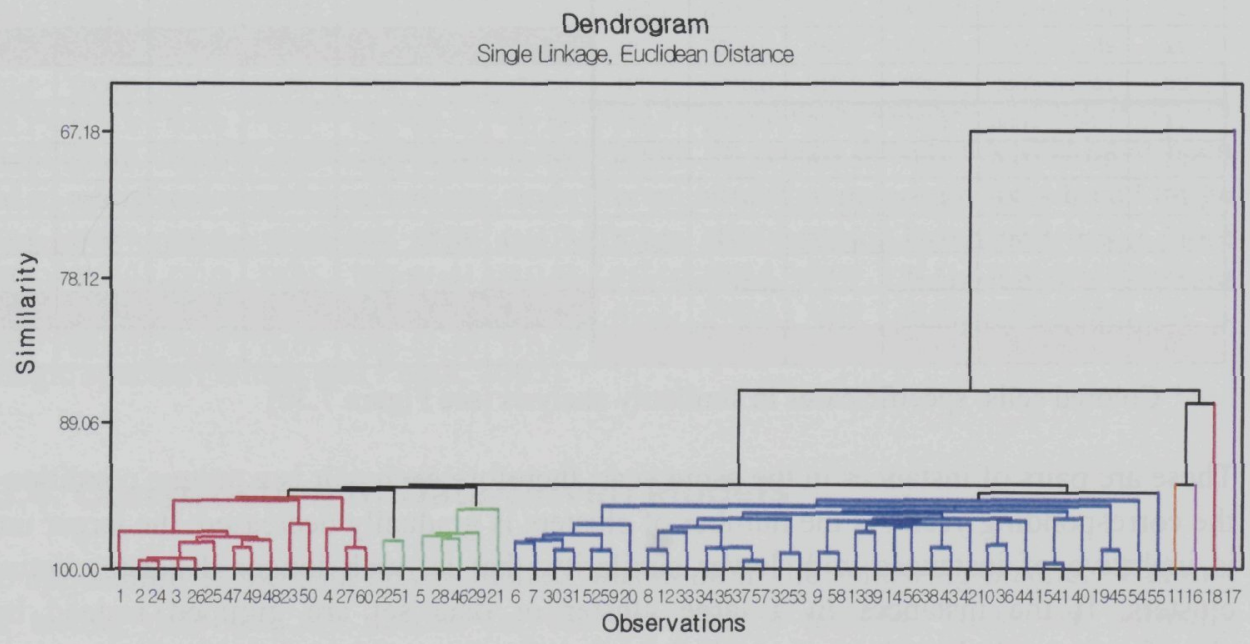

Figure 7.30. Dendrogram of Similarity among Instances from 2005 to 2007.

The instances 11, 16, 17 and 18 have lower similarities than others (see Table 7.5). These four instances were observed only in 2005. Even though the reason for this phenomenon is unknown, we note that 2005 is the first year after the impoundment of the Yongdam reservoir. Therefore it is considered that each nutrient (pollutant) was stored in the reservoir and moved to the dam site with the flood flows. Instances 8 and 12 in 2005, 30 and 31 in 2006, 52 and 59 in 2007 are also very special cases. 
Table 7.5. Water quality data set measured from 2005 to 2007 .

\begin{tabular}{|c|c|c|c|c|c|c|c|c|c|c|c|}
\hline $\begin{array}{c}\text { Instance } \\
\mathrm{s} \text { No. }\end{array}$ & Date & \begin{tabular}{|c|}
$\begin{array}{c}\text { W.Temp } \\
(\mathrm{C})\end{array}$ \\
\end{tabular} & $\begin{array}{r}\mathrm{T}-\mathrm{P} \\
(\mathrm{mg} / \mathrm{L}) \\
\end{array}$ & $\begin{array}{l}\mathrm{PO}_{4}-\mathrm{P} \\
(\mathrm{mg} / \mathrm{L}) \\
\end{array}$ & $\begin{array}{l}\mathrm{Chl}-\mathrm{a} \\
\left(\mathrm{mg} / \mathrm{m}^{2}\right) \\
\end{array}$ & $\begin{array}{c}\text { Instances } \\
\text { No. }\end{array}$ & Date & $\begin{array}{c}\begin{array}{c}\text { W.Temp } \\
\left({ }^{\circ} \mathrm{C}\right)\end{array} \\
\end{array}$ & $\begin{array}{c}\mathrm{T}-\mathrm{P} \\
(\mathrm{mg} / \mathrm{L}) \\
\end{array}$ & $\begin{array}{l}\mathrm{PO}_{4}-\mathrm{P} \\
(\mathrm{mg} / \mathrm{L}) \\
\end{array}$ & $\begin{array}{l}\mathrm{Chl}-\mathrm{a} \\
\left(\mathrm{mg} / \mathrm{m}^{\prime}\right) \\
\end{array}$ \\
\hline 1 & $26-01-2005$ & 5.800 & 0.008 & 0.0000 & 1.133 & 31 & $01-06-2006$ & 19.88 & 0.010 & 0.0032 & 0.374 \\
\hline 2 & $21-02-2005$ & 4.300 & 0.005 & 0.0000 & 0.873 & 32 & $15-06-2006$ & 22.78 & 0.005 & 0.0045 & 0.710 \\
\hline 3 & $03-03-2005$ & 4.500 & 0.009 & 0.0004 & 1.158 & 33 & $06-07-2006$ & 23.31 & 0.012 & 0.0024 & 2.634 \\
\hline 4 & $14-04-2005$ & 11.100 & 0.008 & 0.0010 & 2.837 & 34 & $14-07-2006$ & 21.78 & 0.009 & 0.0005 & 1.786 \\
\hline 5 & $06-05-2005$ & 14.500 & 0.009 & 0.0000 & 1.930 & 35 & $19-07-2006$ & 21.11 & 0.015 & 0.0017 & 2.445 \\
\hline 6 & $19-05-2005$ & 19.200 & 0.007 & 0.0002 & 1.645 & 36 & $27-07-2006$ & 20.38 & 0.021 & 0.0034 & 9.469 \\
\hline 7 & $03-06-2005$ & 19.600 & 0.017 & 0.0000 & 1.074 & 37 & $04-08-2006$ & 21.23 & 0.017 & 0.0011 & 1.666 \\
\hline 8 & $30-06-2005$ & 22.300 & 0.014 & 0.0000 & 2.837 & 38 & $16-08-2006$ & 21.23 & 0.010 & 0.0023 & 6.276 \\
\hline 9 & $07-07-2005$ & 20.300 & 0.030 & 0.0080 & 3.819 & 39 & $24-08-2006$ & 23.13 & 0.015 & 0.0005 & 5.927 \\
\hline 10 & $14-07-2005$ & 20.500 & 0.030 & 0.0030 & 8.813 & 40 & 07-09-2006 & 23.98 & 0.026 & 0.0007 & 7.683 \\
\hline 11 & $21-07-2005$ & 21.300 & 0.014 & 0.0050 & 15.007 & 41 & $14-09-2006$ & 22.65 & 0.024 & 0.0048 & 7.748 \\
\hline 12 & $09-08-2005$ & 22.200 & 0.013 & 0.0040 & 3.290 & 42 & 20-09-2006 & 21.37 & 0.022 & 0.0057 & 4.584 \\
\hline 13 & $18-08-2005$ & \begin{tabular}{|l|}
24.100 \\
\end{tabular} & 0.029 & 0.0060 & 6.144 & 43 & 28-09-2006 & 21.53 & 0.025 & 0.0002 & 5.785 \\
\hline 14 & $29-08-2005$ & 23.500 & 0.016 & 0.0030 & 4.113 & 44 & $13-10-2006$ & 20.7 & 0.006 & 0.0009 & 7.554 \\
\hline 15 & 08-09-2005 & 22.500 & 0.028 & 0.0050 & 7.722 & 45 & $26-10-2006$ & 19.1 & 0.010 & 0.0017 & 5.772 \\
\hline 16 & 15-09-2005 & 23.000 & 0.018 & 0.0030 & 13.513 & 46 & $22-11-2006$ & 14.2 & 0.008 & 0.0000 & 2.841 \\
\hline 17 & $27-09-2005$ & 22.500 & 0.017 & 0.0032 & 31.307 & $47(1)$ & 18-01-2007 & 6.04 & 0.013 & 0.0008 & 0.134 \\
\hline 18 & $06-10-2005$ & 21.100 & 0.014 & 0.0000 & 19.506 & $48(2)$ & $08-02-2007$ & 5.3 & 0.006 & 0.0007 & 0.013 \\
\hline 19 & $17-10-2005$ & 19.600 & 0.024 & 0.0040 & 5.741 & $49(3)$ & $20-03-2007$ & 6.6 & 0.004 & 0.0017 & 0.077 \\
\hline 20 & $25-10-2005$ & \begin{tabular}{|l|}
18.100 \\
\end{tabular} & 0.038 & 0.0030 & 2.216 & $50(4)$ & $05-04-2007$ & 9.1 & 0.009 & 0.0012 & 0.245 \\
\hline 21 & $10-11-2005$ & 16.000 & 0.032 & 0.0073 & 1.293 & $51(5)$ & $25-04-2007$ & 13.3 & 0.005 & 0.0021 & 0.077 \\
\hline 22 & $25-11-2005$ & 12.500 & 0.038 & 0.0057 & 0.151 & $52(6)$ & $25-05-2007$ & 18.5 & 0.007 & 0.0017 & 0.003 \\
\hline 23 & $15-12-2005$ & 8.200 & 0.010 & 0.0020 & 1.091 & $53(7)$ & $05-07-2007$ & 22.9 & 0.010 & 0.0007 & 1.136 \\
\hline 24 & $19-01-2006$ & 4.52 & 0.009 & 0.0005 & 0.872 & $54(8)$ & 02-08-2007 & 24.8 & 0.007 & 0.0014 & 1.123 \\
\hline 25 & $28-02-2006$ & 3.88 & 0.018 & 0.0022 & 0.258 & $55(9)$ & $23-08-2007$ & 25.60 & 0.007 & 0.0010 & 4.200 \\
\hline 26 & $24-03-2006$ & 4.78 & 0.009 & 0.0026 & 0.736 & $56(10)$ & 11-09-2007 & 22.60 & 0.015 & 0.0010 & 4.800 \\
\hline 27 & $24-04-2006$ & 10.71 & 0.018 & 0.0027 & 1.278 & $57(11)$ & $02-10-2007$ & 21.20 & 0.005 & 0.0010 & 1.910 \\
\hline 28 & $11-05-2006$ & 14.21 & 0.015 & 0.0038 & 3.719 & $58(12)$ & $18-10-2007$ & 19.90 & 0.023 & 0.0010 & 3.780 \\
\hline 29 & $18-05-2006$ & 15.20 & 0.011 & 0.0035 & 2.712 & $59(13)$ & $01-11-2007$ & 18.00 & 0.020 & 0.0010 & 0.030 \\
\hline 30 & $25-05-2006$ & 19.37 & 0.008 & 0.0016 & 0.284 & $60(14)$ & $06-12-2007$ & 10.90 & 0.009 & 0.0010 & 1.780 \\
\hline
\end{tabular}

* Colored cells: specific cases in similarity analysis (see Figure 7.30)

These are pairs of instances in the same year; therefore each pair is a unique condition for the corresponding year. As the number of clusters is gradually decreased, the larger union is inclusive of instances with lower similarity, that is, with longer distances between clusters. If the instances in a large cluster or data set are grouped instead by a classification rule based on a certain set of attributes, such a rule severely impairs the regression. The reason for this is that when we predict the Chl-a concentration, the predictors of Chl-a must be grouped into either the partitions or clusters of a trained model even though they have low similarity.

The unusual cases, such as shown in Figure 7.30, are important for data-driven modelling, even though in the past they may have been regarded as being incorrectly measured. Many well known data-driven models and new approaches were tested in this study to find the most relevant technique for eco-environmental modelling. The following section focuses on finding these algorithms. 
The main objective of the data-driven modelling exercise is to find latent information in the form of multiple regressions using machine learning from the data sets. Multivariate regression is classified under two headings: linear and non-linear. Most statistical methods belong to the linear category. Otherwise, knowledge-based regression such as data-driven modeling, data mining and artificial intelligence etc. are non-linear methods.

\subsubsection{Selection of Task-Relevant Model}

Eco-environmental modellers used to adopt a data pre-manipulation technique (e.g., Monte Carlo method, confidence limit, etc) to reinforce the prediction of the multivariate linear regression method (Whitehead and Hornberger, 1984). However, data pre-manipulation can reduce the dimension of the dataset, and it sometimes causes a loss of important and crucial information about the ecosystem. When the focus of the model is on the prediction itself, it is worthwhile to utilize data-driven modelling approaches.

The environmental models should extract latent information as well as predict new information, implying that machine learning approaches for data-driven modelling can be preferred compared to linear regression (Jeong et al, 2006). The reason is that datadriven models have the possibility of treating abnormal or small data sets through nonlinear machine learning techniques.

ANNs, $k N N$, MTs, clustering and PLSR (partial least squares regression) were tested in this study to identify more appropriate algorithms. In recent decades ANNs have been used in many real-world applications, and offer an attractive paradigm for a broad range of adaptive complex systems. $k \mathrm{NN}$ and MTs are also instance-based and use a more explicit learning algorithm such as discussed in Section 5.2.2. Clustering is a different technique from those above. A prime application area for clustering techniques is biologic species (Witten and Frank, 2005).

\subsubsection{Construction of Data-Driven Models}

The application of the data driven models was done in the following manner: Training and testing data sets are needed for the data driven models. These are normally generated by partitioning the input data by cross validation and percentage splitting. In cross validation and percentage splitting, the data is split into $n$ approximately equal partitions (for example 10-fold cross validation splits the data into ten equal partitions) and each partition in turn is used for testing, and the remainder is used for training. Every partition is used exactly once for testing.

However cross validation and percentage splitting reduces the extraction of information due to some data being excluded for training. In addition, the exchange of data sets for training and testing with three years of data is meaningless because the prediction accuracy should be dependent on the machine learning algorithms and not on particular 
combinations of the data. A data combination only changes the number of instances between the training and testing data sets, and the modelling results are influenced by how many abnormal instances belong to either the training or testing data sets. If the combination of each data set impacts on the accuracy of prediction, it means that the modelling performance is influenced by the training data and not by the selected machine learning algorithm. Therefore, the data sets were divided into only two groups: 2005 and 2006 for training and 2007 for testing.

Two different machine learning algorithms, classification and clustering, were used with the regression trees and regression models: LSE and PLS. The application scenarios are summarized in Table 7.6.

Table 7.6. Classification of data-driven modelling and applied models for this study.

\begin{tabular}{c|l|l}
\hline \multicolumn{2}{c|}{ Unsupervised learning } & \multicolumn{1}{c}{ Supervised learning } \\
\hline Clustering & \multicolumn{1}{c|}{ Partitioning } & Regression (Tree \& Numerical prediction) \\
\hline \multirow{3}{*}{$k$-means } & $\begin{array}{l}\text { Standard Deviation } \\
\text { Reduction (SDR) }\end{array}$ & $\begin{array}{l}\text { MTs (M5), ANN, } \\
\text { LSER (Least Square Error Regression), } \\
\text { PLSR (Partial Least Square Regression) }\end{array}$ \\
\hline
\end{tabular}

The Data-Driven Modelling applied for this study: $k N N$, ANNs (MLP and RBF), M5', M5+PLSR, Clustering( $k$-means)+MTs+PLSR, Clustering( $k$-means)+PLSR

\section{1) Artificial Neural Network (ANN)}

Two kinds of ANNs were used in this study for comparison with other competing datadriven models: multi-layer perceptron (MLP) and radial basis function (RBF). An ANN is known as a supervised network because it requires a desired output in order to learn. The goal of this type of network is to create a model which correctly maps the input to the output using historical data, so that the model can then be used to produce the output when the desired output is unknown. In unsupervised learning, there is no target variable, and the network is supposed to train itself to extract "feature variables" from the independent variables; this was not considered in this study.

The algorithm for ANN was provided by Weka software. The number of iterations for training was 700. A classifier of MLP-ANN uses back-propagation to classify instances. The number of hidden neurons was 2 with the number of attributes as shown in Figure 7.31 .

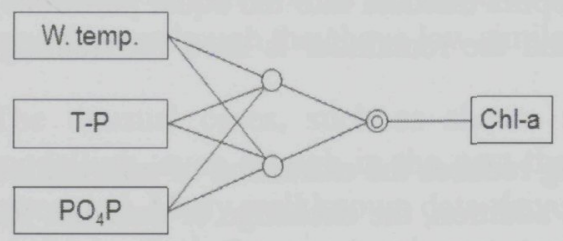

Figure 7.31. Structure of ANN with 2 hidden neurons. 
As RBF-ANN is usually treated as hybrid networks, the $k$-means clustering algorithm is used to provide the basis functions. The inputs are clustered, and the RBF centres are set equal to the cluster means. Activation functions are determined by fitting symmetric multivariate Gaussian equations to the data for each cluster. The optimal number of clusters in reducing the root mean square error of Chl-a was nine in this study.

\section{2) $k$ Nearest Neighbouring ( $k N N)$}

Three instance-based neighbours were used for classification, and the nearest neighbour was sought according to the Euclidean distance. Because the priority constituents for classification are not determined in advance, the nearest neighbour is determined after comparing the distances between or among instances for every constituent according to the value of $k$ ( 3 in this study). Having two or more predicted variables makes it difficult to interpret the results of the instance based classification.

\section{3) Model Trees}

The classifier used for model trees was the M5'(prime) algorithm of Weka software. The regression trees were not pruned and conditions were introduced to compensate for sharp discontinuities between adjacent linear models. Eight instances were used for the final splitting of the trees so that at least 4 instances were available for linear regression on the 4 variables after binary splitting at the final leaf.

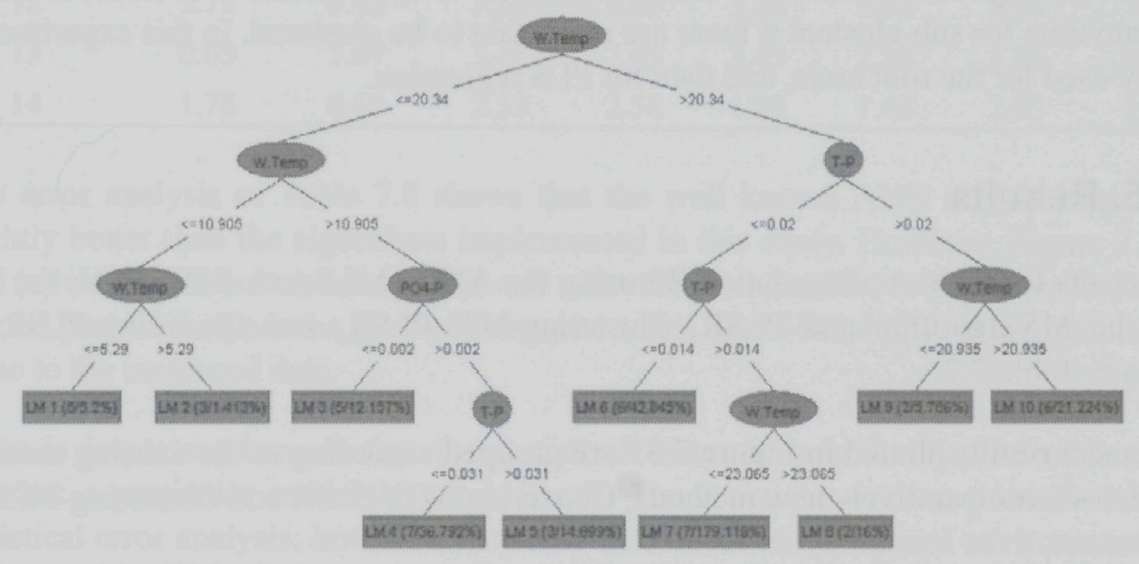

$$
\begin{aligned}
& \text { LM 1: Chl-a }=0.2147 \times \text { W.Temp }-0.5628 \\
& \text { LM 2: Chl-a }=0.2163 \times \text { W.Temp }-0.5554 \\
& \text { LM 3: Chl-a }=0.1899 \times \text { W.Temp }+0.0081 \\
& \text { LM 4: Chl-a }=0.1899 \times \text { W.Temp }+0.0803 \\
& \text { LM 5: Chl-a }=0.1899 \times \text { W.Temp }+0.0294
\end{aligned}
$$$$
\text { LM 6: Chl-a }=0.1528 \times \text { W.Temp }+178.8629 \times \text { T-P }+1.2346
$$$$
\text { LM 7: Chl-a }=0.1528 \times \text { W.Temp }+156.505 \times \text { T-P }+2.2642
$$

$$
\begin{aligned}
& \text { LM 8: Chl-a }=0.1528 \times \text { W.Temp }+156.505 * \mathrm{~T}-\mathrm{P}+2.1297 \\
& \text { LM 9: Chl-a }=0.1528 \times \text { W.Temp }+3.8093
\end{aligned}
$$$$
\text { LM 10: Chl-a }=0.1528 \times \text { W.Temp }+3.7242
$$

Figure 7.32. Structure and linear models of model trees constructed by SDR at sampling station No. R9. 
Finally, 4 instances were chosen for the linear models. This condition was determined after several trials. In the prediction phase of the inference procedure, a new instance is fed into one of the models at the tree leaves, constructed during the tree-building procedure using the splitting condition, and then the predicted values are obtained from the linear models at the leaves. The M5' algorithm uses the least square error (LSE) method for the regression.

In this study, the partial least squares regression (PLSR) method was implemented. This method explains the variations in both the independent and dependent variables. Therefore, in the case of fewer instances than the number of attributes, PLSR can be used for regression in the final leaves instead of the least square error method. The conditions for MTs-PLSR were introduced to M5'(prime), and the algorithm was coded with Matlab.

\section{4) Clustering-Model Trees-PLSR}

For this algorithm, the classic clustering technique called $k$-means was used. Using $k$ means we do not know the number of clusters in advance. The total number of clusters turned out to be nine when each cluster has more than two instances, which is the minimum number for PLSR.

Since clustering is also a kind of learning machine, clustering itself was tested with PLSR. Although the clustering $k$-means technique is used for the root node, it can be used anytime for sub-clusters if there are more data to be clustered. In this experiment it is only used for the root node, and then for PLS regression.

\subsubsection{Results}

The results for the sampling station R9 using the ANNs-MLP and -RBF, $k N N$, the MTs with the M5'algorithm and PLSR, Clustering-MTs-PLSR, and Clustering-PLSR are shown in Table 7.7.

The model results plotted in Figure 7.33 are grouped according to the existing methods and the two comparatively new methods, Clustering-MTs-PLSR and Clustering-PLSR.

The mechanic learning processes are implicit in all cases except for MTs. Almost all of the results showed higher values than the measured values through the year. Despite the shortage of historical data, the modelling performance using 46 training and 14 testing data sets is very reasonable. The fact that there is a larger error from January to August than from September to December is discussed in more detail in the next section.

The error analysis methods applied in this study are as follows. The correlation coefficient (CC) measures the strength and direction of a linear relationship between two random variables (the predicted and observed values). 
The root mean square error (RMSE) measures the discrepancies between predicted and observed values. The root relative square error (RRSE) is relative to what it would have been if an observation had been used. Thus, the relative squared error takes the total squared error and normalizes it by dividing by the total squared error of the observation.

Table7.7. Prediction results of 7 algorithms on Chl-a concentration at sampling station R9.

\begin{tabular}{ccccccccc}
\hline $\begin{array}{c}\text { Instance } \\
\text { No. }\end{array}$ & $\begin{array}{c}\text { Measured } \\
\text { in 2007 }\end{array}$ & $\begin{array}{c}\text { ANNs } \\
\text {-MLP }\end{array}$ & $\begin{array}{c}\text { ANNs- } \\
\text { RBF }\end{array}$ & $k N N$ & M5' & $\begin{array}{c}\text { M5- } \\
\text { PLSR }\end{array}$ & $\begin{array}{c}\text { C-M5- } \\
\text { PLSR }\end{array}$ & $\begin{array}{c}\text { C- } \\
\text { PLSR }\end{array}$ \\
\hline 1 & 0.134 & 0.16 & 0.87 & 1.02 & 0.75 & 0.82 & 0.88 & 0.81 \\
2 & 0.013 & 0.34 & 0.87 & 0.95 & 0.59 & 0.72 & 0.78 & 1.08 \\
3 & 0.077 & 0.36 & 0.87 & 0.91 & 0.87 & 0.89 & 0.97 & 1.25 \\
4 & 0.245 & 0.51 & 1.21 & 1.87 & 1.41 & 1.23 & 1.70 & 2.70 \\
5 & 0.077 & 0.89 & 2.54 & 2.58 & 2.61 & 1.76 & 2.48 & 3.26 \\
6 & 0.003 & 2.14 & 2.79 & 2.97 & 3.59 & 2.45 & 3.73 & 3.78 \\
7 & 1.136 & 4.06 & 2.90 & 2.94 & 6.52 & 6.78 & 4.44 & 3.79 \\
8 & 1.123 & 4.04 & 2.87 & 3.82 & 6.28 & 7.34 & 4.81 & 5.31 \\
9 & 4.2 & 4.44 & 2.87 & 3.83 & 6.40 & 7.57 & 4.97 & 5.81 \\
10 & 4.8 & 5.57 & 6.08 & 4.67 & 8.07 & 12.90 & 3.49 & 3.85 \\
11 & 1.91 & 2.18 & 2.81 & 5.48 & 5.37 & 6.27 & 1.48 & 2.27 \\
12 & 3.78 & 6.42 & 7.53 & 5.01 & 3.79 & 2.63 & 5.44 & 5.44 \\
13 & 0.03 & 3.87 & 3.18 & 2.43 & 3.43 & 2.38 & 1.26 & 0.51 \\
14 & 1.78 & 0.66 & 2.53 & 2.58 & 1.80 & 1.48 & 2.03 & 2.75 \\
\hline
\end{tabular}

The error analysis of Table 7.8 shows that the well known ANN algorithm performs slightly better than the algorithms implemented in this study. However, Figure 7.33 (a) and (c) show different results before and after the instance No. 9. For example, for the second part of the year the prediction results using the C-M5-PLSR algorithm are very close to the measured data.

What is important here is that the statistical error analysis is insufficient to decide whether a particular model is good or not. There can be outliers identified by the statistical error analysis; however, they may also occur in the natural environment. This raises the importance of having an expert to assess the results of the error analyses in eco-environmental modelling.

From Figure 7.30 and Table 7.8, it is easy to discern the best algorithm for ordinary use. However, as the aim of data-driven modelling is to find certain information from the results of the modelling, the selection of the best algorithm requires an insight into the error analysis, the algorithm of the data-driven model and the intrinsic background of the research domain. 
Data-driven modelling results (a)

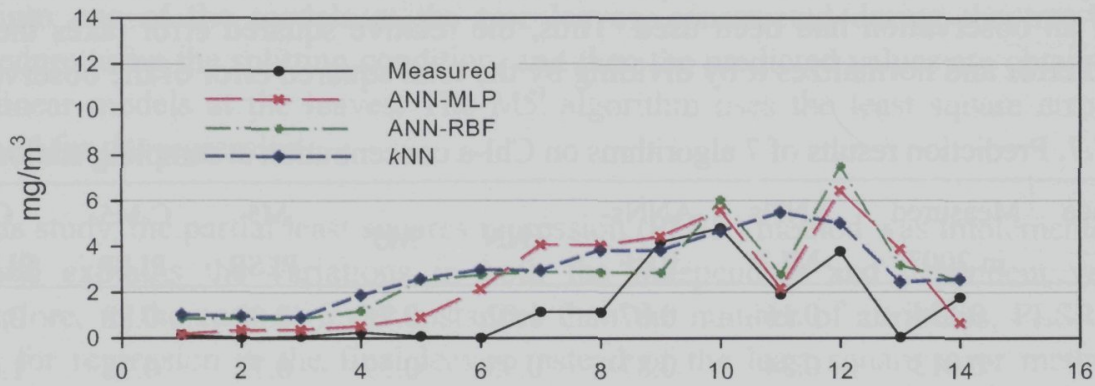

Data-driven modelling results (b)

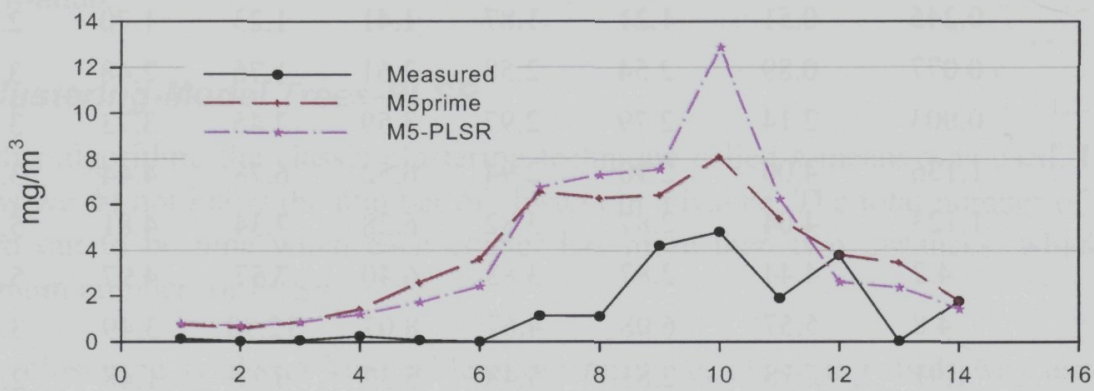

Data-driven modelling results (c)

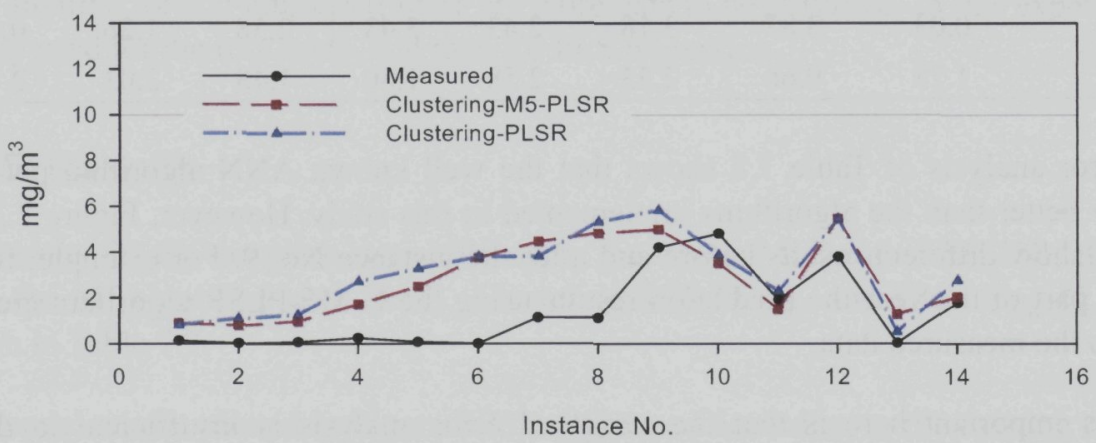

Figure 7.33. Modelling results of Chl-a concentrations using 7 algorithms at sampling station R9: ANNs-MLP and -RBF, KNN, M5' MTs, M5-PLSR, CM5', C-M5-PLSR.

Table 7.8. Prediction error of 7 algorithms on $\mathrm{Chl}$-a concentration at sampling station R9.

\begin{tabular}{cccccccc}
\hline $\begin{array}{c}\text { Terms of } \\
\text { error }\end{array}$ & ANNs-MLP & $\begin{array}{c}\text { ANNs- } \\
\text { RBF }\end{array}$ & KNN & M5 & M5-PLSR & $\begin{array}{c}\text { C-M5- } \\
\text { PLSR }\end{array}$ & C-PLSR \\
\hline CC & 0.74 & 0.75 & 0.74 & 0.69 & 0.73 & 0.61 & 0.66 \\
RMSE & 1.82 & 1.92 & 1.92 & 2.88 & 3.63 & 1.98 & 2.16 \\
RRSE & 1.11 & 1.17 & 1.17 & 1.75 & 2.21 & 1.21 & 1.32 \\
\hline
\end{tabular}




\subsubsection{Discussion}

In data-driven models, one of the most important processes is machine learning, such as classification, clustering and association. Association rules that usually involve only nonnumeric attributes (Witten et al, 2005) were not involved in this study. From Figure 7.33 we can see a different prediction performance and pattern according to the machine learning algorithm used.

The ANN family of algorithms shows a better global fitness than other algorithms. If we want to get information through knowledge based data-driven modelling processes, a more explicit algorithm for machine learning is required to explain and persuade stakeholders. ANNs are more obscure than MTs in terms of their learning processes, because ANNs do not disclose any information about the physical processes that they represent. The main difficulty with ANNs commonly used in practice is perhaps their opacity and the lack of a computationally efficient method to minimize their empirical error. The algorithms are usually based on suboptimal gradient descent heuristics, such that they minimize the mean-square error rather than the empirical error itself. Such algorithms often get stuck at local optima, thus hindering the effectiveness of the training phase (Alippi, 2005).

The ANNs-RBF using the $k$-means clustering algorithm for input to the hidden layer shows a marginally better performance than ANNs-MLP. This means that clustering can be important in eco-environmental modelling. Because ANN-RBF uses clustering, there is usually a bandwidth of activation functions associated with each hidden node. The integrated activation function multiplied with the linear combination of the hidden values can deteriorate the accuracy of the output in the case of a large number of input variables.

The $k \mathrm{NN}$ using the instance-based learning technique shows worse results than the ANNs. The reason may be that the $k N N$ uses specific instances rather than pre-compiled abstractions such as clustering during prediction. Focussing on the nearest scalar value for a particular attribute between instances instead of vectors of instances brings about a dispersion of information embedded in the other attributes of each instance as well. Therefore, when there are more than two variables in an instance, the $k \mathrm{NN}$ is not a full instance-based learning technique.

The MTs give a more explicit knowledge flow through their binary splitting tree structure than ANNs, but their prediction accuracy is less than ANNs. If the number of attributes is more than three, splitting based on the largest standard deviation reduction between only two of the same attributes of neighboring instances causes a dispersion of information as with $k \mathrm{NN}$. Even though MTs have linear models at each final leaf for numerical prediction, the binary splitting in the case of a large number of variables is not the best tree regression rule. Therefore the results of MTs combined with PLSR are worse than the results of ANNs (see Figure 7.33 (b)). 
PLSR seeks more effective latent vectors that explain variations in both the dependent and independent variables on the data set. Therefore the partition (classification) by the SDR of MTs causes a dispersion of information, and then PLSR exaggerates the error by inappropriate partitioning of the data set.

Clustering with MTs was combined with two learning algorithms, namely, classification and PLSR. From Figure 7.33 (c), we can see the level of prediction is different before and after the instance No. 9. This gives a hint as to whether the error is due to the data acquisition, the irregular variation of water quality or the missing data, which is critical to machine learning processes. We cannot see a similar pattern in Figure 7.33 (a) and (b) with (c).

We can therefore deduce the significance of reservoir mixing from this figure: there is an overturn (vertical mixing) by cooling in the autumn and heating in the surface layer and density flow during the summer season. The reservoir undergoes vertical mixing before the strong thermal stratification by heating in the summer; after that the stratified layer is maintained until December when the same water temperature is reached in the whole reservoir by the cooling of the surface water and overturning.

The flood inflow from June to August also disturbs the thermal stratified layer. Therefore the variations of water quality in depth during that period are very unstable. This may be caused by arrange of different inhibitors in the reservoir inflow, and/or mixing conditions, and retention times (Lawrence, 2000). These findings indicate that eutrophication processes in the reservoir, being second order and/or third order activities, were affected by many inhibitors that are not identified by laboratory experiment. The algal growth in this period is very abnormal.

To increase the prediction level, more data representing various natural conditions should be supplied in the future. The prediction results for instances 1 to 3 and from 9 to 14 show very good fit (see Figure 7.33). The reason is that these instances belong to the period when the reservoir maintains a stable status vertically because of the strong thermal stratification before vertical overturning by warming surface water in the spring and cooling it in the autumn, and there are no large inflows disturbing the well stratified reservoir water column.

Although the prediction results of C-M5-PLSR look similar to the results of C-PLSR, the results of C-PLSR are marginally better than those of C-M5-PLSR. This means that the training process combining these two different kinds of learning technique: clustering and partitioning by SDR (standard deviation reduction), is not always recommended for eco-environmental modelling. The reason for this is that the partition (binary splitting) by SDR in C-MTs-PLS algorithms has the possibility of a dispersion of information as discussed above or of over-fitting in the training process. Consequently, PLSR itself can be a good technique for numerical prediction; therefore there is a benefit in combining it with other machine learning techniques, but care must be taken to avoid over-fitting. 
There are differences in the size of the prediction errors according to the learning algorithm used. C-M5-PLSR and ANNs-RBF, which use a clustering technique, are identified as being better than other methods. It can be concluded that these two algorithms are appropriate for eco-environmental modelling, including ecological processes.

The clustering technique actually applies when the instances are to be divided into natural groups, such as according to time or space, rather than when there is no class to be predicted. This reveals that some instances bear a stronger resemblance to each other than with the remaining instances (Witten et al, 2005).

Data-driven modelling for water quality prediction has adopted ANNs in recent years (Jeong et al, 2006; Zaheer et al, 2003; Lindsay et al, 2002; Baruah et al, 2001), and as a consequence there is still a lack of an explicit explanation about the above inhibitors. Using advanced visualization techniques, data and the results of ANN models can be observed together to provide a deeper understanding of how a system behaves throughout the ranges of its variables (Lindsay et al, 2002).

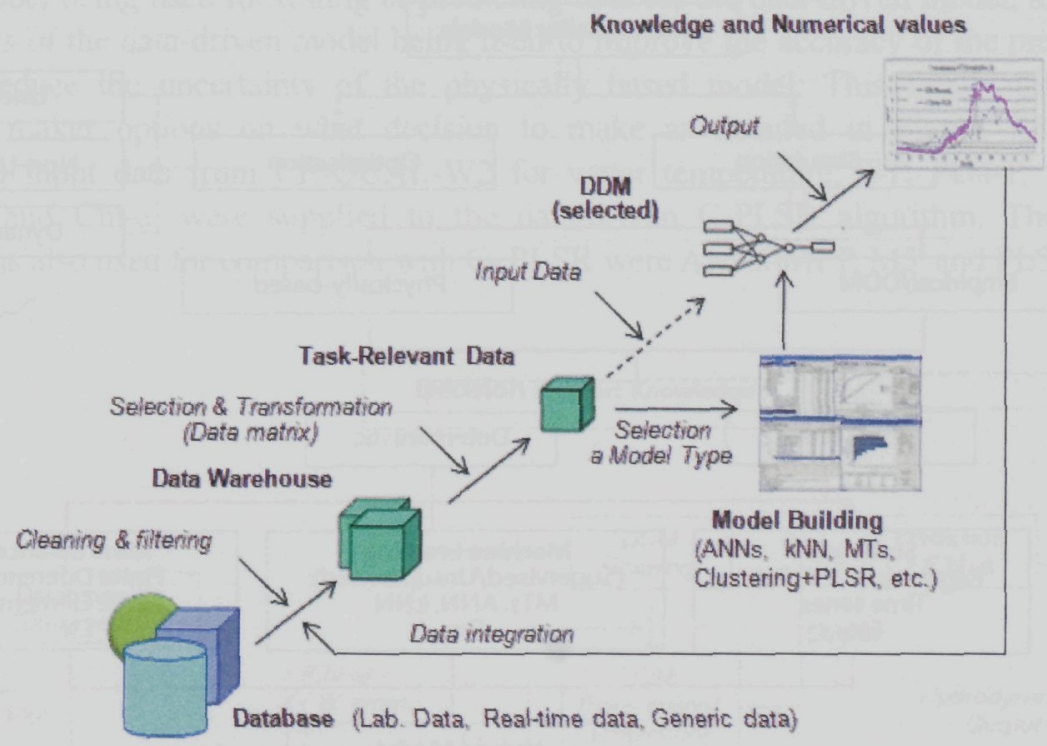

Figure 7.34. Process of DDM (data-driven modelling) implemented for ecoenvironment in this study.

In summary, the recommended algorithms for the Yongdam reservoir include a clustering technique considering seasonal variations and then partial least square regression applied to each cluster. However, it is noted that ANNs-RBF and M5' also show a meaningful results. Consequently, the following process (see Figure 7.34) is implemented for a hybrid model consisting of a data driven and physically-based numerical model. 


\subsection{Hybrid Model}

\subsubsection{Outline}

A hybrid model, as a combination of two or more different models, is designed to achieve a particular objective or goal. The different models implement the fundamental principles identified in Figure 7.35. Here, reservoir simulation models are categorized in two groups: physically-based models and empirical models. Although the two models used in this research are deterministic, which fix the relationship between the input data and output results for the reservoir, the methods are differentiated by having or not having the relevant mechanisms for the intrinsic environmental processes. The natural environment is a very complicated system: it is impossible to fully describe the intrinsic environmental processes with mathematical equations. In this case the empirical models (data-driven models), including statistical and artificial intelligence techniques, are used to mine information about the system. When combining these two kinds of model, the result is a hybrid model.

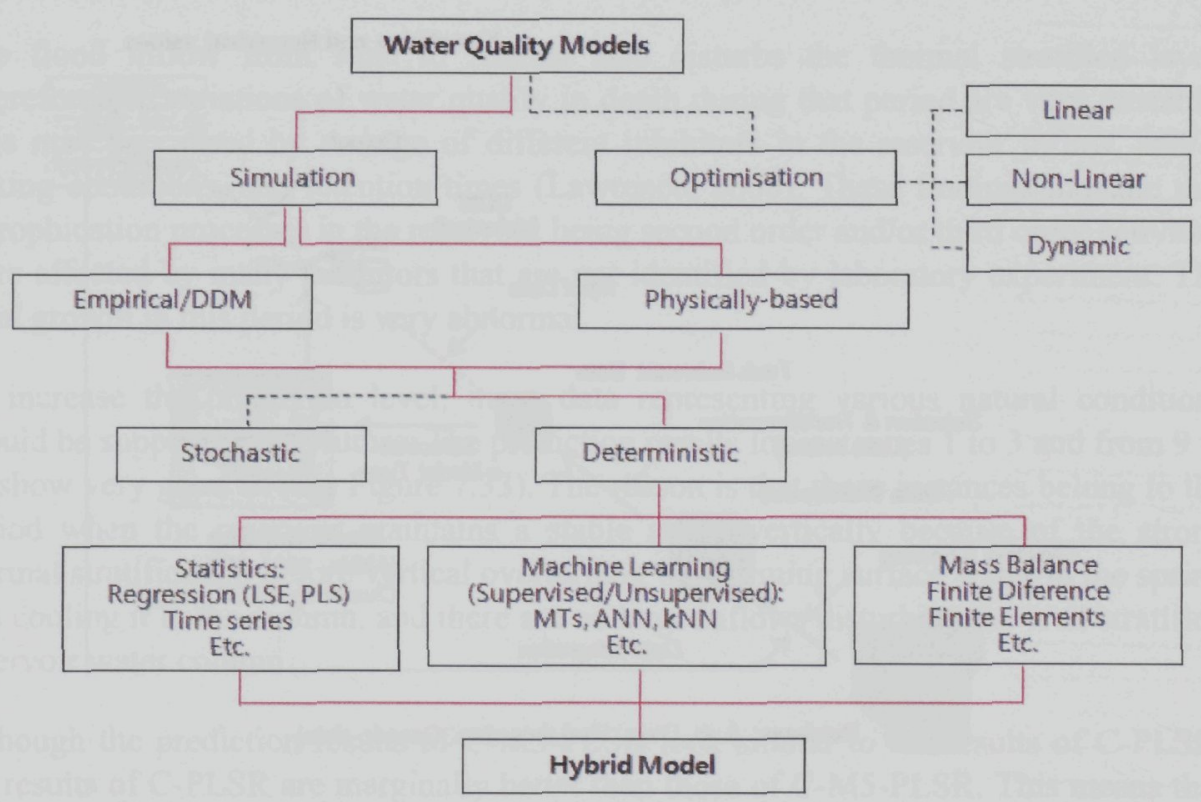

Figure 7.35. Classifications of Water Quality Models (Hybrid model: After James, 1993).

Hybrid models are generally used for incorporation in a decision support system such as for long-term planning (in which it may be used to forecast the increase in algal growth in response to some management decision) or for short-term operational purposes (in which it is used for day-to-day monitoring of algal growth in an already eutrophic reservoir). 
Generally, dependent variables such as water temperature and dissolved oxygen, and their advection and dispersion are simulated with high accuracy in a physically-based model. However, the environmental processes, as an interaction between chemical and biological processes, are still not fully understood in many cases.

According to the results of the physically-based and data-driven models of Sections 7.2 and 7.3, CE-QUAL-W2 has short-comings in representing the biochemical processes for the algal community, and the data-driven models are limited through lack of sufficient data and missing data such as for certain meteorological parameters.

In this study, a hybrid model was implemented to reduce the short-comings of these two kinds of model: the reduction of residual errors for Chl-a in the physically-based model and the generation of daily data from physically-based model for data-driven modelling.

\subsubsection{Integration of Physically-Based and Data-Driven Models}

The integration of the two kinds of model consists in some of the results of the physicallybased model being used for testing or predicting data for the data-driven model, and then the results of the data-driven model being used to improve the accuracy of the prediction and to reduce the uncertainty of the physically based model. This finally gives the decision maker options on what decision to make as detailed in Figure 7.36. The simulated input data from CE-QUAL-W2 for water temperature, T-P, PO4-P, vertical velocity and Chl-a, were supplied to the data-driven C-PLSR algorithm. The other algorithms also used for comparison with C- PLSR were ANNs-MLP, M5' and PLSR.

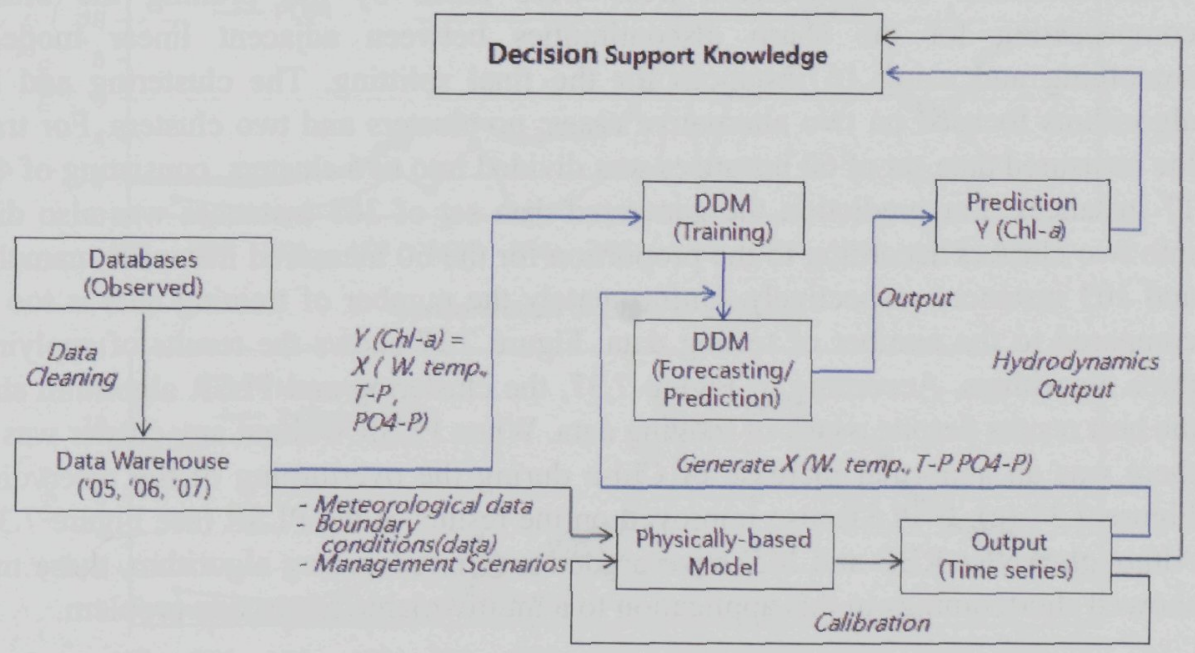

Figure 7.36. Process of model integration for hybrid modeling.

The vertical velocity is one of the most important parameters for deep reservoirs. As discussed in the previous Section 7.2, the Yongdam reservoir has different vertical 
mixing regimes between $\mathrm{R} 9$ and $\mathrm{R} 10$ in the same lacustrine zone due to the intake facilities for water supply. Therefore these two stations were selected to analyse the impact of the vertical mixing velocity on the algal community.

As the vertical velocities were not measured during this study because of a lack of budget and the limitation in the technology, the analysis of their impact on algal community was performed with simulated data from CE-QUAL-W2. Since the results of hydrodynamic modelling are reasonably well fitted to the measured data, this kind of hybrid model is a rational combination of the two kinds of model. Since the Yongdam reservoir does not have sufficient data of a period several years due to its comparatively recent impoundment and the limited research period, the analysis was executed with the simulated data for 2005 and 2006.

\subsubsection{Results}

\section{1) Data-Driven Modelling Using Measured Data and Results of Numerical Modelling.}

Despite the limitation to the small data set of 60 instances from 2005 to 2007, the data set was used for training; otherwise the daily output (365 instances) of the numerical model (CE-QUAL-W2) for the year 2006 was used for testing (prediction) and the generation of the time series data.

The radial basis function network (ANNs-RBF) showed the best fit for 13 clusters using $k$-means clustering. The classifier used for model trees was the M5'(prime) algorithm of Weka software. The regression trees were made by not pruning the branches, compensating for the sharp discontinuities between adjacent linear models by smoothing and using 16 instances for the final splitting. The clustering and PLSR algorithms focused on two alternative cases: no clusters and two clusters. For training the measured data set of 60 instances was divided into two clusters, consisting of 43 and 17 instances. For prediction the generated data set of 365 instances was also divided into two clusters according to the proportion for the 60 measured instances, namely 262 and 103 instances respectively. Unfortunately the number of training data is too small compared to the number of testing data. Figure 7.37 shows the results of applying the three algorithms. According to Figure 7.37, the clustering and PLSR algorithm showed the best results despite a lack of training data. When PLSR without any cluster was used, there was an abnormal increase of Chl-a during the overturning of the reservoir (see Figure 7.37 (c). C-PLSR also improved on the results of the PLSR (see Figure 7.37 (d). Although ANNs-RBF and M5' have a good machine learning algorithm, these models showed shortcomings in this application to a multivariable regression problem.

However, C-PLSR generated some negative values of Chl-a concentration from January to mid of April. The reason for this may be that there are too few training data with very low values and/or PLSR seeks a negative latent vector from the data set during this period. To prevent the negative prediction, more training data should be accumulated 
through increasing the sampling times during winter and spring. The sampling times of these periods are monthly, whereas it is weekly or biweekly for other seasons. A regular sampling interval over the year is more desirable for data-driven modelling.

Concentration of Chl-a in 2006 (a)

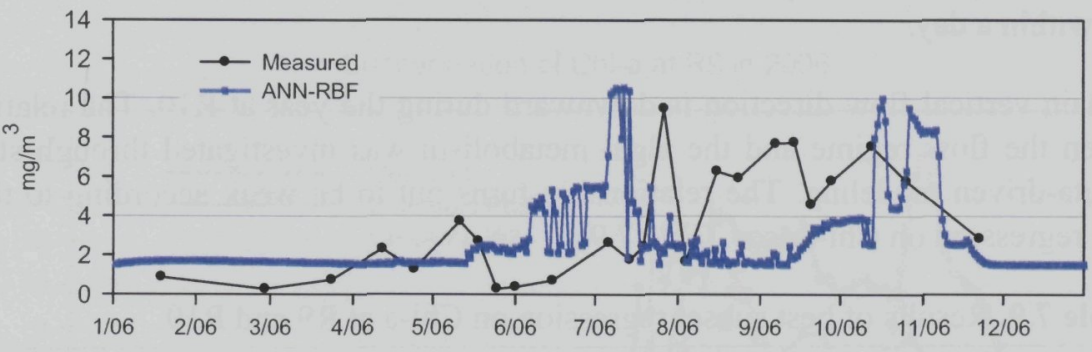

Concentration of Chl-a in 2006 (b)

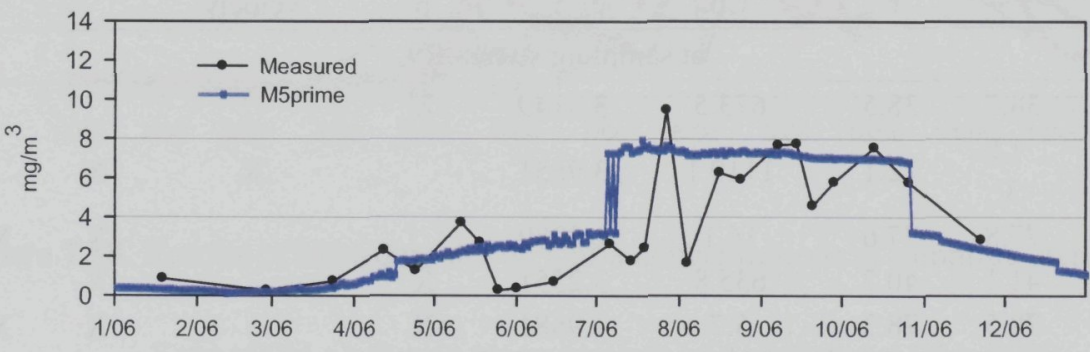

Concentration of Chl-a in 2006 (c)

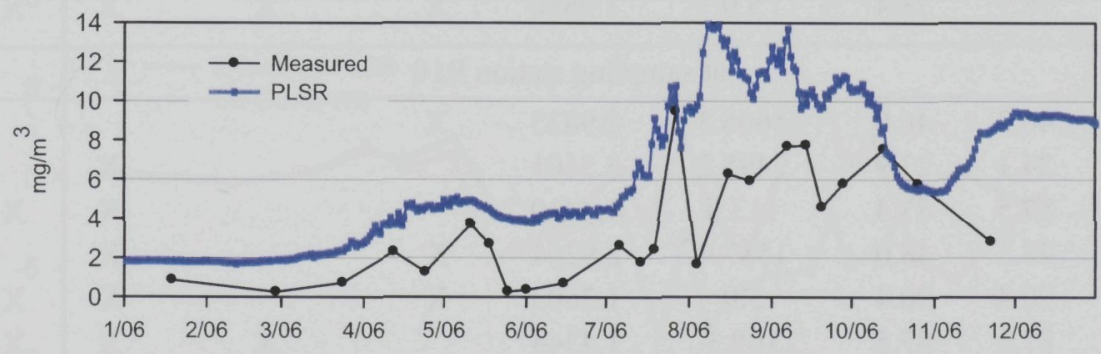

Concentration of Chl-a in 2006 (d)

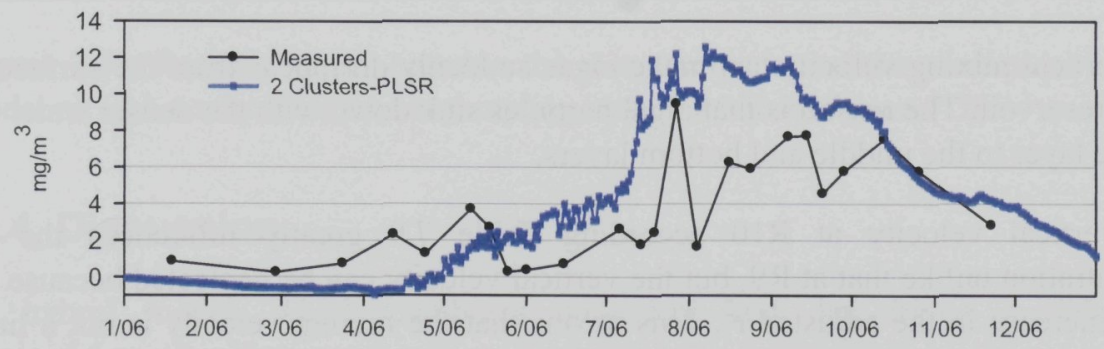

Figure 7.37. Chl-a concentrations at sampling station R9, according to 4 algorithms: ANNs-RBF, M5', PLSR and 2 Clusters-PLSR. 


\section{2) Influence of Vertical Flow Velocity on Algal Metabolism}

In the lacustrine zone, the vertical mixing velocity during overturning generated by differences in the water density is quite large and exhibits significant variations. The average vertical velocities in 2006 are $16.3 \mathrm{~m} /$ day (max. $91 \mathrm{~m} /$ day) at $\mathrm{R} 9$ and $3.2 \mathrm{~m} /$ day (max. $33 \mathrm{~m} /$ day) at $\mathrm{R} 10$ respectively. These velocities can turn the reservoir water upside down within a day.

The main vertical flow direction is downward during the year at R10. The relationship between the flow regime and the algal metabolism was investigated through statistics and data-driven modeling. The relationship turns out to be weak according to the best subset regression on Chl-a (see Table 7.9).

Table 7.9. Results of best subset regression on $\mathrm{Chl}-\mathrm{a}$ at $\mathrm{R} 9$ and $\mathrm{R} 10$.

\begin{tabular}{|c|c|c|c|c|c|c|c|c|}
\hline Vars. & $r^{2}$ & $\underset{r^{2}}{\text { Adjusted }}$ & $\begin{array}{c}\text { Mallows' } \\
\mathrm{Cp}\end{array}$ & $\mathrm{S}\left(\mathrm{mg} / \mathrm{m}^{3}\right)$ & $\begin{array}{c}\text { W.tem } \\
\text { p }\end{array}$ & $\begin{array}{l}\text { Vertical } \\
\text { velociy }\end{array}$ & T-P & $\mathrm{PO}_{4} \mathrm{P}$ \\
\hline \multicolumn{9}{|c|}{ at sampling station R9 } \\
\hline 1 & 38.7 & 38.5 & 673.5 & 3.3149 & $\mathrm{X}$ & & & \\
\hline 1 & $\begin{array}{c}12 . \\
3\end{array}$ & 12.1 & 1119.1 & 3.9651 & & $\mathrm{X}$ & $\mathrm{X}$ & \\
\hline 2 & 77.8 & 77.6 & 16.1 & 1.9989 & & & $\mathrm{X}$ & $\mathrm{X}$ \\
\hline 2 & 41.1 & 40.7 & 635.8 & 3.2551 & $\mathrm{X}$ & & $\mathrm{X}$ & \\
\hline 3 & 78.5 & 78.3 & 5.7 & 1.9681 & $\mathrm{X}$ & & $\mathrm{X}$ & $\mathrm{X}$ \\
\hline 3 & 78.2 & 78.0 & 11.3 & 1.9834 & & $\mathrm{x}$ & $\mathrm{X}$ & $\mathrm{X}$ \\
\hline 4 & 78.7 & 78.4 & 5.0 & 1.9636 & $\mathrm{X}$ & $\mathrm{x}$ & $\mathrm{X}$ & $\mathrm{X}$ \\
\hline \multicolumn{9}{|c|}{ at sampling station $\mathrm{R} 10$} \\
\hline 1 & 46.2 & 46.0 & 1666.3 & 2.9825 & $\mathrm{X}$ & & & \\
\hline 1 & 24.1 & 23.9 & 2495.2 & 3.5401 & & & $\mathrm{X}$ & \\
\hline 2 & 87.5 & 87.4 & 113.0 & 1.4410 & & & $\mathrm{X}$ & $\mathrm{X}$ \\
\hline 2 & 48.3 & 48.0 & 1587.4 & 2.9264 & $\mathrm{X}$ & & $\mathrm{X}$ & \\
\hline 3 & 90.4 & 90.4 & 3.0 & 1.2603 & $\mathrm{X}$ & & $\mathrm{X}$ & $\mathrm{X}$ \\
\hline 3 & 87.6 & 87.5 & 109.3 & 1.4344 & & $\mathrm{x}$ & $\mathrm{X}$ & $\mathrm{X}$ \\
\hline 4 & 90.4 & 90.3 & 5.0 & 1.2620 & $\mathrm{X}$ & $\mathrm{X}$ & $\mathrm{X}$ & $\mathrm{X}$ \\
\hline
\end{tabular}

The vertical mixing velocity can make algae suddenly disappear from the surface layer of the reservoir. The reason is that algal particles sink down with the denser water of the surface layer to the middle and bottom layers.

The vertical velocity at R10 according Table 7.9 greatly influences the Chl-a concentration unlike that at $\mathrm{R} 9$, but the vertical velocity can be neglected because of the small increase in the adjusted $r^{2}$. This means that the mixing velocity is not a limiting factor for the algal metabolism, and therefore it might be only one of the temporary inhibitors for algal growth.

Finally, to prevent negative values Figure 7.37 (d), the data measured at R7 and R10 in 
the same lacustrine zone from January to May for three years are added to the training data set. The simulation results of the data-driven models ( 2 clusters-PLSR) using the results of CE-QUAL-W2 are plotted in Figure 7.38. The simulation results of the hybrid model for the Chl-a concentration show a better fit to the measured data than the results of the numerical model (CE-QUAL-W2).

Concentration of Chl-a at R9 in 2006

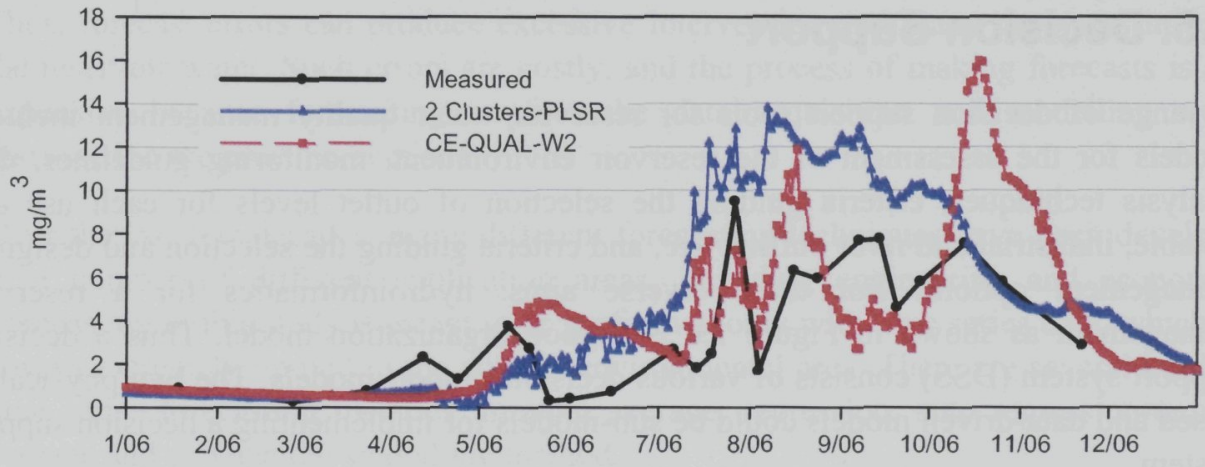

Figure 7.38. Results of hybrid model on Chl-a concentrations at sampling station R9.

Error of Chl-a between measurements and predictionat R9 in 2006

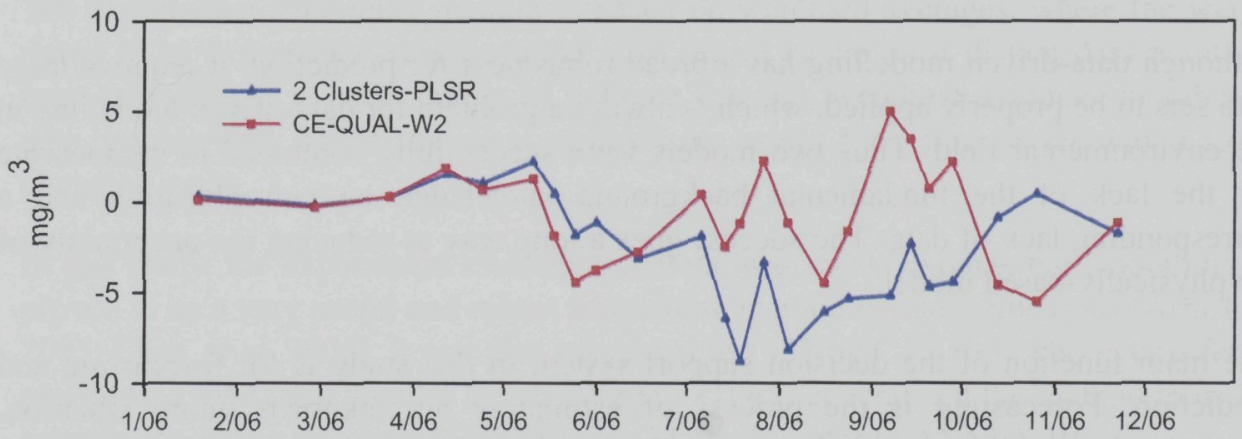

Figure 7.39. Residual errors between the measurements and the predictions of each model on Chl-a concentrations at sampling station R9 from Figure 7.38.

\subsubsection{Discussion}

The hybrid model, which combines numerical and data-driven models, gives meaningful results. There are however, limitations due to the small number of measured data. Nevertheless, the simulation results of Chl-a concentration in 2006 using the time series output of the numerical model and the training results of clustering-PLSR with measured data for 3 years (from 2006 to 2007) testify that the hybrid model is an 
acceptable methodology for the simulation of eutrophication.

The hybrid model also improves the simulation results of the numerical model, CEQUAL-W2, by reducing the uncertainty in the algal metabolism function of the model. Consequently the hybrid model improves the simulation results of the Chl-a concentration and produces a good set of time series data.

\subsection{Decision Support}

A range of decision support tools for reservoir water quality management includes models for the assessment of the reservoir environment, monitoring guidelines, data analysis techniques, criteria guiding the selection of outlet levels for each use e.g. potable, industrial and river outflow etc, and criteria guiding the selection and design of management options. For these diverse aims, hydroinformatics for a reservoir management as shown in Figure 1.6 is a good organization model. Thus a decision support system (DSS) consists of various decision support models. The two physicallybased and data-driven models could be sub-models for implementing a decision support system.

The physically-based and data-driven models each have many advantages in themselves. However despite the surprising progress of computer science there are still limitations in physically-based modelling such as a fundamental lack of background knowledge of the ecological processes and burgeoning computing times.

Although data-driven modelling has a broad robustness for prediction, it requires large data sets to be properly applied, which is always a problem for data-driven modelling in the environmental field. Thus two models were successfully combined to compensate for the lack of the fundamental background knowledge on eutrophication and a corresponding lack of data. The success goes a long way to reducing the uncertainty of the physically-based model.

The main function of the decision support system in this study is for forecasting and prediction. Forecasting is the process of estimating an unknown future situation. Prediction is a similar process, but it is different in its usage. Usage can differ according to the areas of application: for example, in water quality management forecasting is sometimes reserved for estimates of values at certain specific future times, while the term "prediction" is used for more general estimates, such as reservoir response due to environmental changes that will occur over a midterm or a long period.

Therefore forecasting focuses on the usage of reservoir water; prediction is directed towards the control of the reservoir water quality with action plans. In this study a forecast of Chl-a concentration and a prediction of reservoir response with an appropriate action plan to control eutrophication in the Yongdam reservoir was implemented through a hybrid model. 


\subsubsection{Forecasting}

Forecast accuracy for algal growth in the reservoir is important because a high forecast leads to other inventories (activated carbon, more chemicals for oxidation and more often backwash of filters) to remove algal particles and odour generated by their decay in the water treatment plant, and a low forecast leads to complaints from customers and to economic loss due to the need to drain the treated water from the water supply system. Thus, forecast errors can produce excessive intervention and many problems in using the reservoir water. Such errors are costly, and the process of making forecasts is also expensive because forecasting requires the data acquisition and modelling works discussed in the previous sections.

Over the last few decades, many different forecasting techniques have been developed in a number of different application areas, including engineering and economics. Forecasting in this study is meant to be performed only with time series data, which are always extremely limited in the aquatic environmental area. There are several kinds of time series forecasting methods: moving average, regression, auto regression-moving average and exponential smoothing method.

The moving average method simply averages the last $m$ observations. The regression method approximates the model by constructing a linear equation that provides the least squares fit to the last $m$ observations.

The exponential smoothing method is based on weighted averages, where the weights decay in an exponential way from the most recent to the most distant data point. The term 'smoothing' is used simply to indicate that this weighted average smoothes the data irregularities. These statistical methods are useful for a time series with a slowly changing mean.

In this study, the exponential smoothing method was used. Exponential smoothing has proven to be a very useful and robust forecasting method in many situations (Armstrong, 2005; Elias, et al. 2006). It was first suggested in 1957 by C.C. Holt who later offered a procedure (1958) that handles trends. Winters (1965) generalized the method to include seasonality, hence the name "Holt-Winters Method" (refer to ANNEX III).

Forecasting is possible because the variable being forecasted has a pattern repeated over time. According to the auto-correlation analysis, the recurrence duration of algal growth is about 15 days with a correlation coefficient greater than 0.5 .

The time series forecasting results for the Chl-a concentration in 2006 was plotted for a short-term forecast of 15 days from Julian day 151 to 165 as shown in Figure 7.36. Three measures of accuracy of the fitted model: MAPE (Mean Absolute Percentage Error), MAD (Mean Absolute Deviation), and MSD (Mean Squared Deviation), were calculated giving the following values: MAPE 117, MAD 0.526 and MSD 0.739. For all three measures, the smaller the value, the better the fit of the model. 
The results shown in Figure 7.40 are quite reasonable for forecasting Chl-a concentration. This forecast smoothes the historical time series data by the Holt-Winters exponential smoothing method and provides short to medium-range forecasts with periodic cycles. The eutrophication processes in reservoirs have a short term cycle of about two weeks and a seasonal cycle. Therefore it is possible to forecast water quality for a short-term period with a historical data set. If the real-time data were accumulated continuously, its accuracy would be increased correspondingly.

Time series forecasting for Chl-a concentration Multiplicative Method

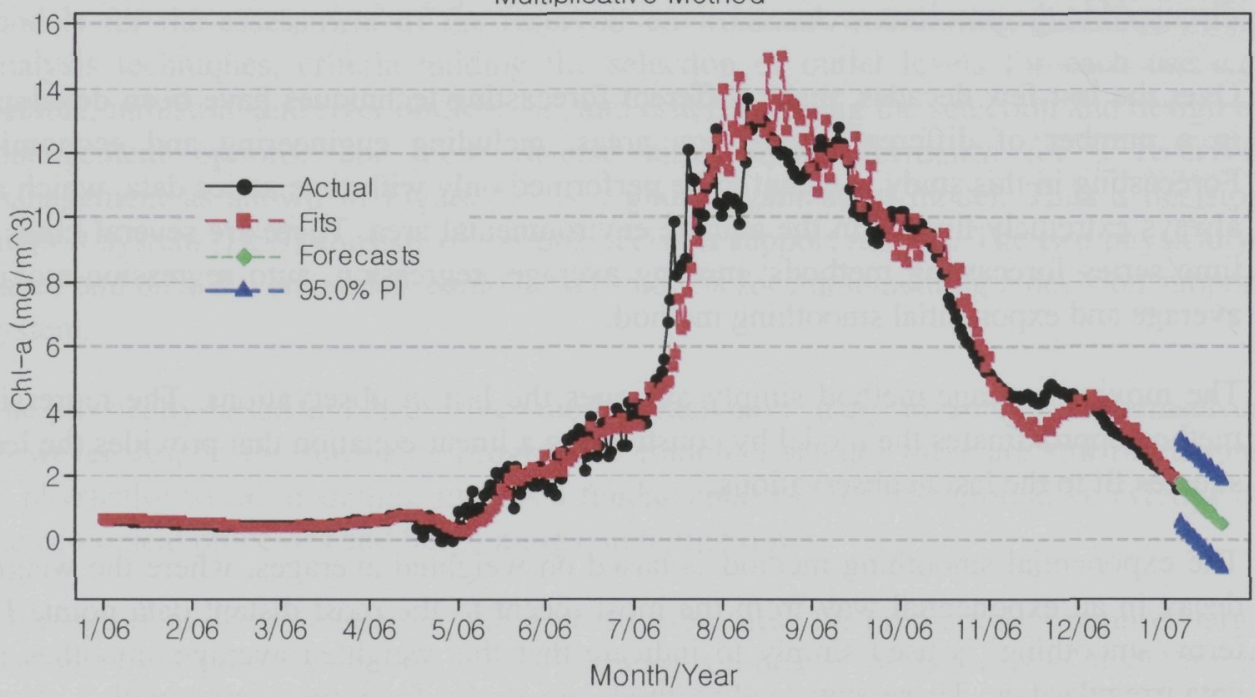

Figure 7.40. Forecasting 15 days for $\mathrm{Chl}$-a concentration using historical data.

\subsubsection{Prediction}

The prediction of the reservoir water quality is actually required for planning the reservoir management strategy. In this study, the methodology was developed, but the actual plan should be the subject of a subsequent study with the acquisition of further measured data.

According to cross-correlation analysis, it is impossible to draw a relationship between nutrient loads upstream in the reservoir and the lacustrine zone given by R9 and R10. Therefore the nutrient concentration in this zone was simulated by the physically-based numerical model. The generated data were used for the data-driven model.

Pollutants of the Yongdam reservoir are mainly from non-point sources transported by rainfall-runoff and from point sources due to three waste water treatment plants (WWTPs) located in the catchment. The WWTP located on Jinan Stream is near to the 
reservoir and is very poor in phosphorus removal; the other WWTPs have a similar performance (see Figure 6.3).

To keep the Yongdam reservoir water quality from deteriorating, several plans/scenarios can be implemented. In this study, only the effects of the nutrient (phosphate) reduction of the most polluted tributary (Jinan) and main stream on the Yongdam reservoir water were analysed with the constructed hybrid model. As the calculation of the nutrient concentration at the sources is outside the scope of this study, only the loading concentrations of nutrient at each tributary were input to the model.

Generally pollutants from point sources can be treated much more easily than non-point sources. Therefore a maximum phosphate reduction of $90 \%$ was assumed for the WWTP and $30 \%$ for non-point source. When there are reductions of $10 \%, 50 \%$ and $90 \%$ in the phosphate concentration in the Jinan Stream (T3), and 10\%, 20\%, and 30\% in the main stream (T2), the Chl-a concentrations at R9 is predicted as shown in Figure 7.41.

The high phosphate concentration (average: $70 \mathrm{mg} / \mathrm{m}^{3}$ ) at $\mathrm{T} 2$ which is higher 3.5 times than T1 (average: $20 \mathrm{mg} / \mathrm{m}^{3}$ ) is caused by the WWTP located just upstream of the reservoir. The pollutants in the main stream due to the other two WWTPs may also be high, but the effluent water from the two WWTPs has some self-purification while flowing to the reservoir. The scenario of the combined reduction of phosphate at T2 and T3 was also applied to the hybrid model. The results of the analysis are shown in Figure $7.41(\mathrm{c})$.

According to Figure 7.41 (a), the high concentration of phosphate from T3 affects marginally the Chl-a concentration at R9. This means that although the phosphate concentration in the river water at T2 is higher than those at T1 and T2, the total phosphate load to the reservoir occupies relatively small portion. The high phosphate concentration at $\mathrm{T} 3$ is because of smaller flow rate (1/4.78 times of $\mathrm{T} 2)$ due to the smaller catchment area compared to other 4 sampling stations.

Consequently, the phosphate reductions of $90 \%$ in the $\mathrm{T} 2$ and of $30 \%$ in the $\mathrm{T} 3$ show that Chl-a concentration of less than about $12 \mathrm{mg} / \mathrm{m}^{3}$ which is below the eutrophic level for OECD standard $\left(8-25 \mathrm{mg} / \mathrm{m}^{3}\right)$ over the year. To obtain a more confident conclusion the research should be continued. This result is not averaged over the whole water column, but confined to a he point of 5-meter deep from the reservoir water surface.

According to Figure 7.41 (c), the reduction rates of Chl-a concentration at R9 are low compared to the pollutant reduction rates at $\mathrm{T} 2$ and $\mathrm{T} 3$. This means that the quantity of phosphate for algal growth are plentiful in the surface layer of the Yongdam reservoir. Research should be continued, and focused on the assessment of the nutrient recycle in the watershed of Yongdam reservoir and on more extensive data acquisition in terms of the sampling interval and vertical depth in the reservoir for better forecasting and prediction. 
Chl-a at R9 in 2006 (a)
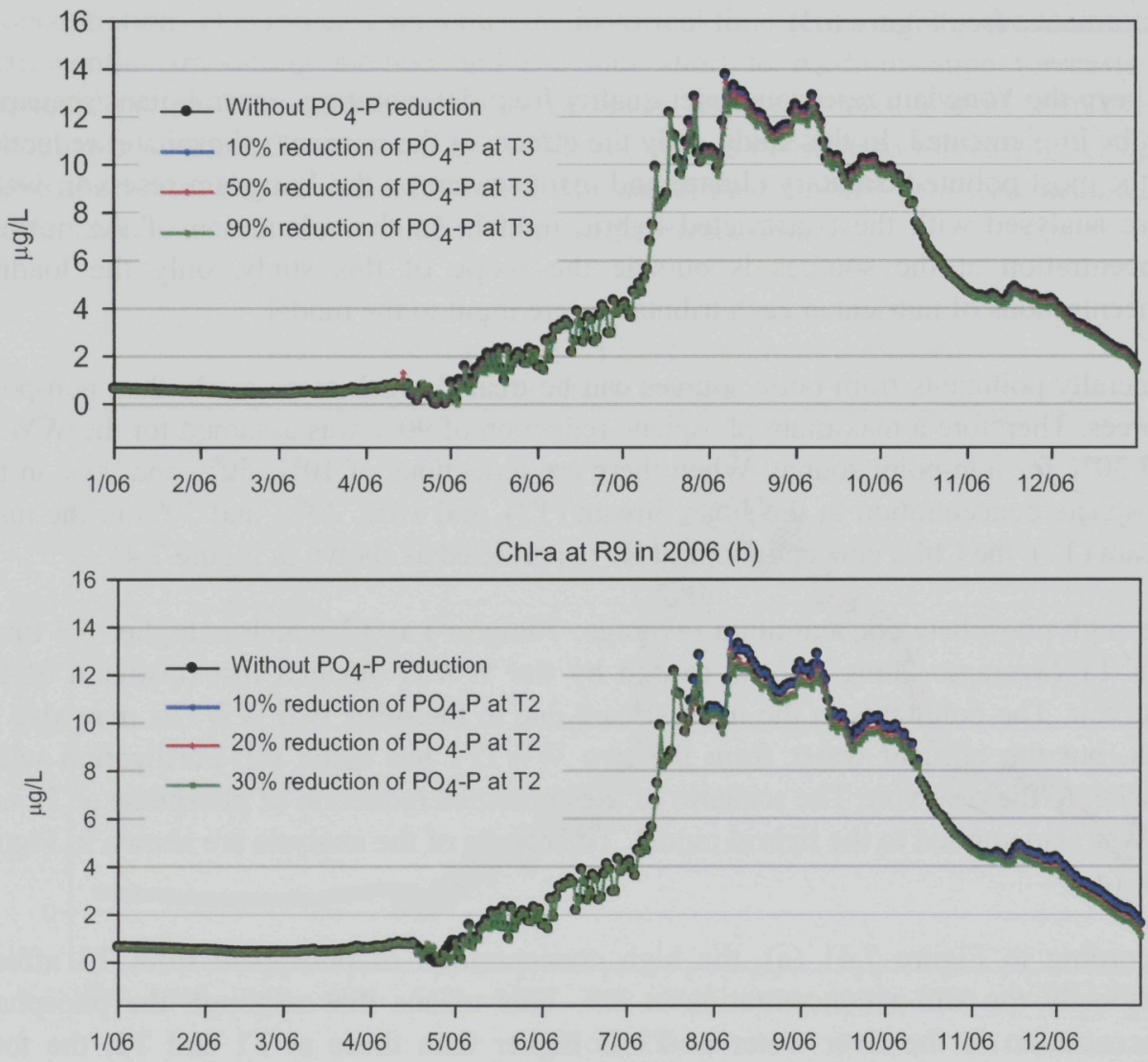

Chl-a at R9 in 2006 (c)

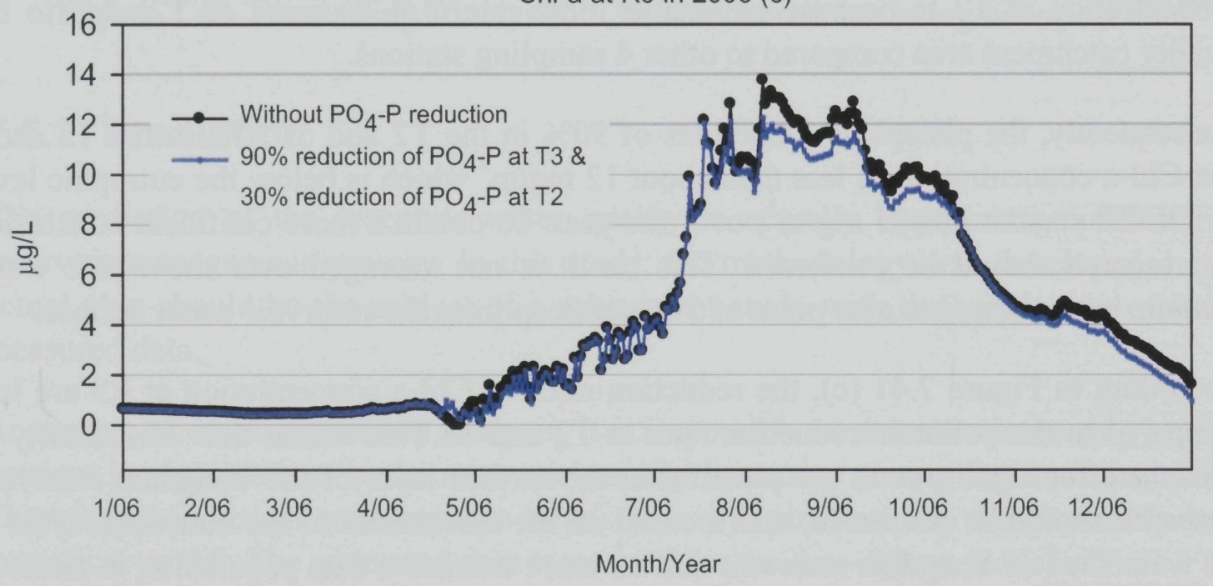

Figure 7.41. Chl-a concentrations at R9 due to phosphate reductions at T2 and T3. 


\section{Chapter 8}

\section{CONCLUSIONS \& RECOMMENDATIONS}

\section{Summary}

This research has contributed to the development of hybrid modelling by combining physically-based and data-driven models for studying eutrophication in reservoirs. Data representing the eco-system of the Yongdam reservoir were monitored and analysed using the hybrid modeling approach. Some conclusions and recommendations from this study are discussed in this chapter. Firstly, the assessment of the reservoir water quality is modelled using the hybrid approach because it is difficult to determine the limiting nutrient for phytoplankton growth using physically-based modelling alone. Secondly, a laterally averaged two-dimensional physically-based model simulates reasonably well the global mixing and the physical-chemical water quality within Yongdam reservoir, which is deep, narrow and thermally stratified,. Thirdly, whereas most three dimensional models such as MIKE 3D have been developed for oceanographic applications, they can be used for the simulation of small scale mixing in the reservoir. Fourthly, it has been demonstrated that the clustering rule and PLS regression is suitable for the case of a small number of data instances compared to the number of variables. Finally, the hybrid model can predict with acceptable accuracy the Chl-a concentration using data generated by the physically-based model. The model was used as a tool for predicting the Chl-a concentration due to nutrient reduction scenarios in the catchment. The recommendations coming out of this study are focused on the Yongdam reservoir and the contributions to reservoir modelling. For more refined eutrophication modelling of the reservoir, shorter sampling intervals at smaller increments in depth are recommended to improve the results of the modelling. Further, better accommodation of the small scale turbulence mixing with physical-biological coupled hydrodynamics is needed for an improved understanding of the reservoir ecosystem. Also, modelling at small spatial scale can be applied to optimize the reservoir operation for the withdrawal of turbid water.

\subsection{Conclusions}

\subsubsection{Reservoir Water Quality Management}

Reservoir eutrophication management along with the control of pollutants in the reservoir is closely related to the local geology, land use, and meteorological conditions in the watershed feeding the reservoir. The nature and anthropogenic uses of the watershed can result in abnormal biological production and ecological successions in the reservoir. This study emphasises the fact that the reservoir water quality management cannot be separated from the watershed management. 
Integrated management is needed in order to control eutrophication in a reservoir. The principle players in the management of the watershed, such as government agencies, private industries, non-government organizations and public bodies (Heathcote, 1998) need to work together in a well organized framework that consists of specialists in wide varying disciplines: engineering, bio-chemical aquatic sciences, economics, sociology, law and ethics.

The framework is typically structured to accommodate the four basic aspects of Hydroinformatics for water management, namely the real, virtual, procedural and societal worlds as shown in Figure 1.5. A prerequisite for best management practice of eutrophication in a reservoir is appropriate attention given to each of these four 'worlds' within the watershed area.

The societal world, with its use of agent-based, economic and social modeling, plays a very important and visible role in the reservoir management community. Many policy discussions may take place in formulating plans regarding the control of pollutants, as well as the establishment of guidelines and/or standards for reservoir management. Therefore the societal world should lead to the development of a master plan involving the assessment of a reservoir and its watershed, the issues, directions and goals to be attained, along with an action plan to be implemented by the society.

This study on the reservoir eco-hydraulic modelling can be considered as a component of the virtual world. It will support decision making through the simulation, forecasting and prediction of eutrophication processes for the Yongdam reservoir. The hybrid modelling technique combining physically-based and data-driven modelling is seen as a promising technology in the construction and development of a decision support system (DSS) for the management of eutrophication in reservoirs.

\subsubsection{Reservoir Ecosystems}

This study shows that a reservoir has to be regarded as a unique eco-system despite many similarities to natural lakes. The outflow of a reservoir is generally controlled at the dam impounding the water, and withdrawals are made at different depths according to the water usage, such as augmenting the discharge downstream of the dam or the intake for drinking water supply. These operational conditions can influence the entire reservoir ecosystem and can generate unique phenomena in each reservoir. Because a reservoir has its own ecological processes due to its watershed, physical dimensions (length, depth and width) and transport mechanism, its ecosystem and physical processes should be regarded as an individual ecosystem. In this study, the important features of the ecosystem for the Yongdam reservoir are identified as follows.

The Yongdam reservoir experiences a double thermocline and turbid density flows due to the summer monsoon, and has two turnovers in spring and autumn. Three different ecosystems can be identified for zones along the reservoir according to its morphology 
and the mixing phenomena. Turbid inflow from the watershed takes place at or above the thermocline rather than mixing with the deep water, which subsequently affects the mixing behavior of the reservoir during the spring and autumn turnovers. The limiting nutrient for algal growth is phosphorus, but nitrogen is limited in the bottom layer of the upstream zones of the reservoir for a short period during the summer.

\subsubsection{Data Acquisition}

Research on reservoir water quality requires two categories of data for the water quality parameters: domain and boundary condition data according to the methods used. Traditionally, statistical methods and data-based inductive models use all the available domain data for training and verification, whereas physically-based deductive models use boundary data for model input and domain data for model calibration and verification. Therefore, each method requires data for different parameters at different monitoring positions and intervals.

Physical boundary data such as inflow and water temperature at each tributary, the outflows and meteorological conditions, are direct forcing functions for reservoir mixing. These data are measured every hour or so, whereas boundary and domain data for water quality are sampled and analysed at considerably longer intervals, e.g. weekly, or monthly because of the limitations of laboratory analysis and the time taken. It can be concluded that water quality modelling suffers more than hydrodynamic modelling by the longer intervals between data and circumstances such as missing samples during flood events at tributaries. The longer intervals between data for water quality parameters reduces the ability to record the variation of water quality between monitoring intervals, whereas missing samples make it difficult for a physically based model to calculate accurately the load of non-point source nutrients accumulated in the watershed area and transported to the reservoir during flood events.

\subsubsection{Water Quality Assessment}

The Yongdam reservoir commenced filling with water in 2001. The eutrophication processes have stabilized over the first three years, namely 2002-2004. This is called an aging process. It means that nutrients of various point and non-point sources not removed from the submerged area before the reservoir impoundment are being diluted and flushed every year. Despite the decreasing patterns of Chl-a and phosphorus concentration during the study period (2005-2007), the organics produced from the watershed and algal decay are increasing at the bottom of the reservoir. The increasing trend of COD in the entire reservoir and the decreasing trend of DO in the bottom layer over the three years have given rise to this conclusion.

In spite of the short period of records (2005-2007), the Yongdam reservoir has experienced different flow regimes in time e.g. a double thermocline and turbidity current in 2005 and 2006 and a single thermocline and no turbidity current in 2007. 
These were due to different meteorological conditions, and particularly rainfall patterns. The weather conditions influenced not only the mixing of the reservoir water affecting the thickness of the thermocline, which influences the nutrient transport and advection, but also the algal growth. According to the algal growth potential test (AGPT) carried out in this study the Yongdam reservoir water has an algal growth potential in addition to the visible algae indicated by the measured Chl-a concentration. This means that some phosphorus, which is the limiting nutrient in that not all the phosphorus is used by the algae, always remains in the reservoir water. This nutrient gives further possibility of algal blooms whenever the condition for algal growth is satisfied.

Consequently, the Yongdam reservoir is slowly going through a limnological evolution from the point of view of the water quality analysis during the three years of the study (2005-2007).

\subsubsection{Eco-hydrodynamic Modelling}

This study shows that a laterally averaged two dimensional model can be successfully applied for the environmental modelling of reservoirs and lakes. The main reason for this appears to be that, unlike the major 3D models, they are designed specifically for reservoirs and lakes. The success of the $2 \mathrm{D}$ model is attributed to its ability to capture the large scale mixing of the reservoir, even though it hardly depicts the small scale turbulent motions. Secondly, the 2D model is much more efficient in its use of computational memory and time

Although the overall circulation of large reservoirs and lakes is characterized by complicated spatial and temporal variations in the flow, advances in computational fluid dynamics have lead to a wide range of three-dimensional models adopting finite volume methods (FVM). Many of these models have had successful applications in oceans and estuaries but very few in lakes/reservoir (Umlauf et al, 1999). The reason for this is that features of the flow in reservoirs are different than in the ocean.

In reservoirs, the advection of pollutants is affected by internal waves, thermal gradients, winds and river inflows, whereas in the ocean, the tidal and Coriolis forces and atmospheric wind and pressure gradients are the primary forcing functions. In addition, reservoirs located in temperate zones experience large variations in water level and in the depth and strength of their thermoclines, which also differ from those in the ocean. Therefore, in order for the three dimensional models to capture the mixing of reservoirs they need to address the small length scales associated with the flows in reservoirs compared to those in the ocean

According to this study, the three dimensional model MIKE 3D, using the FVM method with flexible mesh, does not simulate the thermocline effectively during summer, whereas the model using the FDM method with a staggered grid gives better simulation results for the mixing in the Yongdam reservoir than the two dimensional model. 
However, MIKE 3D is expensive to run for long time series and therefore the computational time and memory need to be reduced in order to model various reservoir management scenarios. Grid cells need to be allowed to wet and dry during a simulation. This feature also needs to be improved in order to include extra grid points for application in areas of high fluctuation in water level such as required by the Yongdam reservoir.

The simulation modelling of the ecological water quality parameter (Chl-a) gives worse results than corresponding physical parameters. There can be several reasons for this: the algorithmic expressions for the ecological processes in the reservoir are limited, there are major shortcomings in the inability to describe small scale turbulence processes that affect the metabolism of microorganisms in the reservoir water, and there is a lack of water quality data for the boundary inputs compared to the flow and meteorological data.

\subsubsection{Data-Driven Modelling}

Data-driven models using a machine learning algorithm such as partition and instance based rules require a considerable amount of data (more than 10,000 in certain cases) in order to generate good information (Witten \& Frank, 2005). However, such a database is nearly impossible to compile from measurements in the eco-environmental research area. Whereas the traditional methods of statistics, such as regression, require a normally distributed data set to ensure a particular confidence level, water quality data are gathered only about 30 times per year (Pentecost, 1999). This is a limited number considering the requirement for a normally distributed eco-environmental data.

Eco-environmental data generally have a seasonal variation, and many parameters are highly correlated with other parameters compared to a noisy set of electronics data or even rainfall-runoff data. This means that a machine learning algorithm for ecoenvironmental data-driven modelling (DDM) should function so that instances bearing a strong mutual resemblance can form clusters, not partitioning but by comparing the entropy (probability of high similarity) of two values of the same parameter.

An eco-environmental DDM with a small number of instances and a high dimension (number of parameters) can make it impossible to induce an appropriate regression equation. In this case the PLSR method tested in this study can find latent vectors that explain both the response and predictor parameters. However, the PLSR does have a short-coming namely that it gives an irregular prediction if there are weak correlations between parameters.

For this reason, the data-driven model for eco-environment modelling should be further developed through augmenting the machine learning algorithm. In this study, the combination of clustering and PLSR shows better results than the machine learning of model tree using its regression method and partitioning rules. 


\subsubsection{Hybrid Model}

The hybrid model combining physically-based and data-driven models is effective and profitable when the two models scientifically and rationally satisfy the needs of each other, such as sharing generic data for the data driven model and providing a solution to overcome not knowing the algorithms for the ecological processes in the physicallybased model.

Since eutrophication has many unknown processes, the Chl-a concentration is predicted by a data-driven model using the generic data from a physically-based model. The calculation of Chl-a concentration by the data-driven model, which is trained with the measured data, can reduce the uncertainty due to unknown factors for the algal metabolism. Although the data-driven model is trained with measured data that is at irregular time series, it can generate $\mathrm{Chl}-\mathrm{a}$ concentrations as a regular time series using daily generic data from the physically based model for purposes of forecasting and prediction. These are strong points of a hybrid model, which combines two models with different inference methods.

The prediction results of Chl-a concentrations using this hybrid model show a better fit to measured data than the results of the physically-based model alone. In addition, the results of the hybrid model can be used to forecast water quality variations in the reservoir and to generate the long term predictions of the reservoir response to remedial actions to improve water quality. This study shows that the hybrid model can be used in the construction of a decision support system for the Yongdam reservoir.

\subsubsection{Achievement of This Study}

The Decision Support System (DSS) for the reservoir water management is aimed at decision making for the evaluation of a strategic water management policy and for operating the reservoir according to various management scenarios. This study is part of the method of decision support using eco-hydraulic modelling of eutrophication for reservoir management. The results of the data acquisition, its analysis and the models constructed in this study (physically-based, data-driven and hybrid) can be regarded as components of the decision support systems: model-driven decision support system, data-driven decision support system and knowledge-driven decision support system (see Chapter 1.5.3).

The AGPT and results of eco-hydraulic modelling of eutrophication showed that soluble reactive phosphorus $\left(\mathrm{SRP}, \mathrm{PO}_{4}-\mathrm{P}\right)$ is the control variable; the abstraction and/or release of the eutrophic water and the reduction of the nutrient load from the watershed are interventions for eutrophication management of the Yongdam reservoir. However the two and three dimensional hydraulic models using a much coarser rectangular grid of 90 meters than the lateral length of the opening size of intake tower or are laterally averaged do not permit the calculation of withdrawal of eutrophic water in detail. The 
two-dimensional water quality model is relevant for simulation of water quality in the long and deep reservoir except for ecological variables such as Chl-a concentration.

The best subset regression on Chl-a before the application of Data-Driven Models (DDMs) showed that TP and PO4P as the nutrient constituents, and water temperature as the physical constituent, explain about $90 \%$ of the variations of Chl-a concentration (see Table. 7.4). Although an eco-environmental DDM with the three dependent variables above is used with the clustering rule and the PLSR algorithm that were confirmed as the best fitted, the data acquisition at less than 30 times per year showed a drawback in deducing eco-environmental information.

Measurements and models in this study are suitable to evaluate EQOs and EQSs for reservoir management, but the abstraction and release of increasingly eutrophic water computational fluid dynamic codes and velocity measurements in three dimensions demand a high technology and budget in a deep reservoir.

It is possible with DDMs to deduce new information and to construct non-linear regression equations with the ability to use the patterns and rules stored in the model to predict trends in multi-dimensional data set. However DDMs do not guarantee the nature of future interventions and control variables. Instead, DDMs are guidance tools, used to provide insight into trends inherent in the historical information.

Finally, the achievements of this study need cooperation between data acquisition and analysis and modelling. Therefore the construction of a decision support system for the Yongdam reservoir is recommended as an integration of the above models and water quality assessment into one system including the data management system.

\subsection{Recommendation for Further Research}

\section{1) Data Acquisition}

For a reservoir management study, one of the most important and difficult issue is the sampling of the reservoir water and the corresponding analysis of the sampled water. Researchers often realize at the final stage of their research that their data acquisition plan was not well formulated. From this study, a few recommendations for establishing a better monitoring strategy are given as follows:

- The data acquisition plan depends on the nature of the model to be used, namely whether it is an inductive or deductive model: the deductive model needs a shorter monitoring interval for the boundary condition data than the monitoring interval for the initial and calibration data.

- Water quality parameters and monitoring points should be selected through principal component analysis to ensure shorter sampling intervals taking into account budgetary constraints. The number of samples (instances) is the most important factor for datadriven modeling of a multivariate eco-environmental process. 
- Chl-a and limiting nutrient concentrations for eutrophication control should be monitored from 0.5 meter depth below the water surface to the bottom of the reservoir at a depth of every meter to identify the vertical biological activities, phytoplankton growth and nutrient gradients due to thermal stratification and density currents.

\section{2) Eco-Hydraulic Modelling}

For future research, there are two kinds of modelling to be considered: small scale turbulence modelling related to the eco-physiological aspects of phytoplankton, and the optimization of the reservoir operation due to the selective discharge of turbid water.

At small length scale (such as the Kolmogorov length scale), water turbulence has a direct effect on the eco-physiology of phytoplankton: the turbulence interacts with the transport of water molecules into and out of the phytoplankton cells and causes mechanical cell damage and morphological adaptations, or it affects contact rates (and hence coagulation and predation processes) between suspended plankton. In the case of motile cells such as red algae with flagellator, their trajectories in the water column are modulated by both the organisms swimming behaviour and the water motion (Berdalet et al, 2005). Modelling these phenomena requires collaboration between physicists, chemists and biologists.

A heavy storm event causes turbidity inflows, which then develop a high turbid density current in the reservoir. The currents overlap due to successive storm events during the monsoon rainy season. Therefore research is recommended on using a small spatial scale modeling scheme for optimizing the discharge rate and divergence of the gate to vent continuous turbidity inflows.

\section{3) Pollutants and Turbidity Control}

The reservoir ecosystem is dependent on its watershed and meteorological conditions. The inflows from the spring and first summer monsoon floods are the primary means for conveying into the reservoir pollutants accumulated on the surface of the catchment area during the dry season and soil eroded by rainfall.

Since the meteorological conditions are not controllable, many in-reservoir remedial technologies such as an algaecide and destratification devices are developed to improve reservoir water quality. However they are temporal measures. When thermal stratification and turbid density flows take place, artificial destratification generates other problems involving the extension of turbidity to the entire reservoir

The first recommendation is given to that point and non-point pollutants should be reduced in the watershed area to prevent or delay eutrophication processes of the reservoir water quality. In addition, pre-impoundment reservoirs or compartmentalization of the reservoir at its upper reaches may be a recommendable alternative to trap nutrients and soil particles in the inflow water. For these remedial works, the hybrid model developed in this study can be used to decide the quantity of pollutant to be cutoff in the watershed area which will satisfy the EQOs by the EQSs set 
up for the Yongdam reservoir.

According to this study the flood inflow from upstream of the reservoir reaches the dam site after 6 days, the selective withdrawal of turbid water from below the surface layer on clean days after rainfall also impacts the downstream ecosystem of the reservoir for a considerable period after the monsoon storms and can result in protests from residents living downstream region. Generally, selective withdrawal discharge must be limited compared to the flood inflow discharges to keep a high efficiency in partitioning clean and turbid water because of access velocities from upstream and downstream of the selective discharge gate and to store the storm water which will be used during the dry season. Therefore, the two dimensional model constructed in this study identifies the selective discharge depth and quantity of dense turbid water, and then the water will be discharged $\mathrm{b}$ successive storm events. 



\section{APPENDIX I \\ Modelling Software}

A model is a pattern, physical entity, or mathematical formulation that can represent an actual system, scenario, or object. Due to the fact that most models are commonly used in the predictive mode, they often differ in time and spatial scales from the objects.

Table A.1. Classification of model due to the order of dimension, grid type and method of numerical solution

\begin{tabular}{|c|c|c|c|c|}
\hline \multirow{2}{*}{ Division } & \multicolumn{2}{|c|}{ 1-D } & \multicolumn{2}{|c|}{ 2-D } \\
\hline & Longitudinal & Vertical & Laterally averaged & Depth averaged \\
\hline Strengths & $\begin{array}{l}\text { Appropriate for } \\
\text { run-of -the-river or } \\
\text { re-regulation } \\
\text { reservoir }\end{array}$ & $\begin{array}{l}\text { - Simplify } \\
\text { assessments of } \\
\text { eutrophication- } \\
\text { related processes } \\
\text { and effects } \\
\text { - Particularly } \\
\text { useful for } \\
\text { simulation water } \\
\text { temperature and } \\
\text { water quality in } \\
\text { deep reservoirs }\end{array}$ & $\begin{array}{l}\text { - Box model approach } \\
\text {-great flexibility } \\
\text { - Hydrodynamic and } \\
\text { mass transport } \\
\text { models } \\
\text { - simulation effect } \\
\text { of water quality } \\
\text { variation on fluid } \\
\text { flow through the } \\
\text { effect on water } \\
\text { density }\end{array}$ & $\begin{array}{l}\text { - Flexible grid(finite } \\
\text { element grid) } \\
\text { - Also formed the } \\
\text { basis of a number of } \\
\text { sediment transport } \\
\text { models }\end{array}$ \\
\hline Weaknesses & $\begin{array}{l}\text { - Vertical and lateral } \\
\text { variation can be } \\
\text { neglected }\end{array}$ & $\begin{array}{l}\text { - Longitudinal and } \\
\text { lateral variation } \\
\text { can be neglected }\end{array}$ & $\begin{array}{l}\text { - Can not simulate } \\
\text { processes that } \\
\text { involve interactions } \\
\text { between along-river } \\
\text { and cross-river } \\
\text { processes }\end{array}$ & $\begin{array}{l}\text { - Can not simulate } \\
\text { density-driven } \\
\text { hydrodynamic } \\
\text { processes } \\
\text { - Can not simulate } \\
\text { wind driven } \\
\text { dispersion }\end{array}$ \\
\hline Models & CE-QUAL-RIV1 & $\begin{array}{l}\text { WQRRS } \\
\text { CE-QUAL-R1 }\end{array}$ & CE-QUAL-W2 & $\begin{array}{l}\text { RMA } \\
\text { MIKE21 }\end{array}$ \\
\hline \multicolumn{5}{|c|}{ 3-D } \\
\hline Strengths & \multicolumn{4}{|c|}{$\begin{array}{l}\text { - State-of-the-art models on hydrodynamics, water quality, cohesive sediments, and } \\
\text { ecology in rivers, lakes, reservoirs, estuaries, bays and seas } \\
\text { - Most flexible and broadly applicable category of hydrodynamic model } \\
\text { - Powerful pre-, post- processing software and visualization software }\end{array}$} \\
\hline Weaknesses & \multicolumn{4}{|c|}{$\begin{array}{l}\text { - Computationally intensive } \\
\text { - Generally unsuitable for direct coupling with biogeochemical models if systematic } \\
\text { and robust automatic parameter estimation or calibration techniques }\end{array}$} \\
\hline Grid Type & $\begin{array}{l}\text { Curvilinear } \\
\text { boundary fitted } \\
\text { grid, } \sigma \text {-coordinate. }\end{array}$ & Arakawa C-grid & $\begin{array}{l}\text { Arakawa C-grid/ } \\
\text { spherical grid } \\
\text { Flexible mesh ( } \sigma \text { - } \\
\text { coordinate) }\end{array}$ & $\begin{array}{l}\text { Curvilinear/ non- } \\
\text { equidistant } \\
\text { rectilinear and } \\
\text { spherical. Vertical } \\
\sigma \text {-coordinate }\end{array}$ \\
\hline $\begin{array}{l}\text { Numerical } \\
\& \\
\text { Time Variable } \\
\text { Solutions }\end{array}$ & $\begin{array}{l}\text { FDM/FVM, } \\
\text { Centred and } \\
\text { Upwind scheme. } \\
\text { Two or three time } \\
\text { level semi-implicit } \\
\text { temporal solution }\end{array}$ & $\begin{array}{l}\text { FVM } \\
\text { Third-order } \\
\text { upwind-weighted } \\
\text { based scheme } \\
\text { QUICK and } \\
\text { QUICKEST } \\
\text { scheme }\end{array}$ & $\begin{array}{l}\text { FDM (ADI)/FEM/ } \\
\text { FVM } \\
\text { UPWIND, } \\
\text { QUICKEST, } \\
\text { SHARP \& } \\
\text { ULTIMATE } \\
\text { schemes }\end{array}$ & $\begin{array}{l}\text { FDM (ADI)/FVM. } \\
\text { Explicit in time and } \\
\text { with a central } \\
\text { discretisation in } \\
\text { space }\end{array}$ \\
\hline Models & EFDC & ELCOM & MIKE3 & DELFT3D \\
\hline
\end{tabular}


Most physically-based modelling software use the partial differential Navier-Stokes equations of motion describe fluid motion and pressures at a point; differential equations also describe the transport of sediment, salinity and other constituents together with interactions between constituents. Due to complex solution of analytical solution of the partial differential Navier-Stokes equations, numerical computation of hydrodynamics and constituent fate is used over the complex geometry of natural water bodies with varying boundary conditions such as behaviour of water bodies. Advances in computer power made such numerical simulations increasingly feasible. Numerical solution methods of the partial differential equation have been progressed into the reduction of numerical diffusion such as ADI, TVD, ENO and WENO etc. The first step in instantiating a model is to discretise the solution domain (the water body) in one, two, or three dimensions, representing the system as one-, two-, or three-dimensional network. The next step is to define the quantities to be modelled (currents, water levels, constituent levels, etc) across the domain discretised (grid). The following introduction focuses on the above model description

Mathematical models designed to represent the transport pathways, fate and results of nutrients in the aquatic environment can serve as powerful tools in understanding and decision making for various management plans. These aquatic environmental processes include transport pathways and fate of naturally occurring constituents, such as solids and nutrients, and contaminants in a watershed are driven by complex interactions of precipitation, land uses, urban and rural watershed runoff, groundwater transport, wastewater and storm water inputs, surface water transport, kinetic transformations and biological processes in the water column and sediment bed.

Therefore, there are not a mathematical model simulating all processes above at the same time because of limited capacity and time for computing. Since most developers of mathematical models focus on a specific event and do not have interdisciplinary knowledge, models also have strong and weak points together. The degree of complexity of models, required to understand and make a decision for various plans for protection of a water body, ranges from simple, screening level approaches based on limited data to detailed investigations driven by extensive data. Therefore, a model selection step before instantiating of model is very important. Here were free and commercial software for lake/reservoir environmental modelling summarized as following. The criteria for the selection depend on objectives identified in each research topic and data availability etc.

\section{1) BATHTUB/FLUX/PROFILE}

BATHTUB is a steady state model that predicts eutrophication response based on empirical formulas developed for nutrient balance calculations and algal response. The model requires nutrient loading inputs from the upstream watershed and atmospheric deposition, morphometric data for the lake, and estimates of mixing depth and non-algal turbidity. Since three interrelated programs (FLUX, PROFILE and BATHTUB) simplify assessments of eutrophication-related processes and effects, the model should be used to assess general trends in water quality rather than to exactly predict inlake 
concentrations of nutrients or chlorophyll a (from TMDL Development for the Beaver Creek and Grand Lake St. Marys Watershed, Ohio).

\section{2) CE-QUAL-W2}

CE-QUAL-W2 (W2) is a water quality and hydrodynamic model in 2D (longitudinalvertical) for rivers, estuaries, lakes, reservoirs and river basin systems. W2 models basic eutrophication processes such as temperature-nutrient-algae-dissolved oxygen-organic matter and sediment relationships.

W2 simulates longitudinal-vertical hydrodynamics and water qualities in stratified and non-stratified systems such as multiple algae, epiphyton / periphyton, zooplankton, macrophyte, CBOD, and generic water quality groups, internal dynamic pipe/culvert model, hydraulic structures (weirs, spillways) algorithms including for submerged and 2-way flow over submerged hydraulic structures, dynamic shading algorithm based on topographic and vegetative cover. W2 also has a limits like as well-mixed in lateral direction (but can be used in a Quasi-3-D mode by use of additional model branches), hydrostatic assumption for vertical momentum equation (from User Manual).

\section{3) HEC-5Q}

HEC-5Q model is an extended model of HEC5, which simulates water quantity, to simulate water quality in stream reaches and reservoirs. HEC-5Q, Simulation of Flood Control and Conservation Systems (Including Water Quality Analysis) computer model, has the unique capabilities to accept user-specified water quantity and quality needs system-wide and to decide how to regulate the network of reservoirs. Therefore $\boldsymbol{H E C}$ $5 Q$ 's reservoir module simulates only the $1 \mathrm{D}$ vertical gradients while longitudinal and lateral profiles are averaged (from U.S. Army Corps of Engineers' Hydrologic Engineering Center (HEC)).

\section{4) WQRRS (Water Quality for River-Reservoir System)}

The WORRS package has been assembled to evaluate water quality (ecologic cycle) conditions in river and reservoir systems. The program provides vertical profiles of water quality conditions in reservoirs and longitudinal conditions in river networks of branching channels and/or around islands. This model was capable of analyzing 18 different physical, chemical, and biological water quality parameters in a river or reservoir or a river reservoir system. A preprocessor was developed by the HEC to simplify preparation of input data and these two programs together were then called the "Water Quality for River-Reservoir Systems" (WQRRS) model. This type of modeling is referred to as 1D modeling. The package includes three separate modules that are able to be integrated into a system analysis or to be used as separate programs; the reservoir module, the stream hydraulic module, and the stream quality module (from Water Quality For River-Reservoir Systems User's Manual October 1978 US Army Corps of Engineers Hydrology Engineering Center).

\section{5) WASP7}


The Water Quality Analysis Simulation Program (WASP7) is an enhancement of the original WASP (Di Toro et al., 1983; Connolly and Winfield, 1984; Ambrose, R.B. et al., 1988). This model helps users interpret and predict water quality responses to natural phenomena and manmade pollution for various pollution management decisions. WASP is a dynamic compartment-modeling program for aquatic systems, including both the water column and the underlying benthos. WASP allows the user to investigate 1D, 2D, and 3D box systems, and a variety of pollutant types. The time varying processes of advection, dispersion, point and diffuse mass loading and boundary exchange are represented in the model with steady, daily, seasonal and yearly time steps. WASP also can be linked with hydrodynamic and sediment transport models that can provide flows, depths velocities, temperature, salinity and sediment fluxes.

WASP is used routinely throughout the United States in the development TMDLs and waste load allocations. The model contains algorithms for conducting: (1) Eutrophication/Conventional Pollutants, (2) Organic Chemicals/Simple Metals, (3) Mercury, (4) Temperature, Fecal Coliforms, Conservative Pollutants. (from US EPA webpage: http:/www.epa.gov/athens/wwqtsc/html/wasp.html)

\section{6) EFDC}

Environmental Fluid Dynamics Code (EFDC) is 2D and 3D orthogonal curvilinear grid hydrodynamic model which solves conservative, $2^{\text {nd }}$ order accurate finite difference equation by finite volume method. EFDC can solve for the circulation and transport of material in complex environments including, estuaries, coastal embayments, lakes and offshore, but not eutrophication. EFDC also provides solutions for salinity, temperature, and conservative tracers with full density feedback to handle stratified conditions. (from US EPA webpage: http://www.epa.gov/athens/wwqtsc/html/efdc.html)

\section{7) RMA (Resource Management Associates)}

RMA is a multidimensional finite element model for simulating estuary hydrodynamics that allows for $3 \mathrm{D}$ formulation of the flow domain. The model solves three dimensional shallow water equations, including baroclinic effects. The system of equations includes the horizontal momentum equations in two directions, the vertically integrated continuity equation, the salinity transport equation, and an equation of state (from California, Estuarine, Coastal, and Shelf Science, Vol. 36, 235-280).

\section{8) ELCOM-CAEDYM}

ELCOM(Estuary and Lake Computer Model)is a three-dimensional hydrodynamics model used for predicting the velocity, temperature and salinity distribution in natural water bodies subjected to external environmental forcing such as wind stress, surface heating or cooling. ELCOM is designed to facilitate modelling studies of aquatic systems over time scales extending to a few weeks, though the limit of computational feasibility depends on the size and resolution requirements of an application and computational resources. ELCOM is suited for comparative studies of the summer and 
winter circulation patterns, spring versus neap tidal cycles, or dispersal conditions under different flow regimes. ELCOM either can be run in isolation for hydrodynamic studies, or coupled with CAEDYM for simulation of biological and chemical processes.

CAEDYM (The Computational Aquatic Ecosystem Dynamics Model) is an aquatic ecological model that may be run independently or coupled with hydrodynamic models DYRESM(1D) or ELCOM(3D). CAEDYM consists of a series of mathematical equations representing the major biogeochemical processes influencing water quality. At its most basic, CAEDYM is a set of library subroutines that contain process descriptions for primary production, secondary production, nutrient and metal cycling, and oxygen dynamics and the movement of sediment. CAEDYM configuration is flexible so that the user can focus on the processes of interest. For example, the model can be configured for a simple set of nutrients-phytoplankton-zooplankton. By simulating several state variables at the species level, CAEDYM can be used to support the understanding and management of a system. In addition, the model can be coupled to the one-dimensional hydrodynamic model (DYRESM) for studies of the seasonal, annual or decadal variation in water quality. For more detailed spatial information, CAEDYM can be run with the three-dimensional hydrodynamic model ELCOM. To maximize speed and memory requirements CAEDYM shares a common internal data structure with both DYRESM and ELCOM. They also use common output data storage formats, and share common Graphical User Interface (GUI) and visualization routines for configuring the model and displaying the results. DYRESM-CAEDYM is presently being used in 24 countries around the world. It can be downloaded as freeware. (from Centre for Water Research (CWR) The University of Western Australia. http://eco.wiz.uni-kassel.de/model_db/mdb/elcom.html)

\section{9) DELFT3D}

Delft3D is a $2 \mathrm{D}$ and $3 \mathrm{D}$ modelling system to investigate hydrodynamics, sediment transport and morphology and water quality for fluvial, estuarine and coastal environments. In 3D simulations, the vertical grid is defined following the so-called sigma coordinate approach. This results in a high computing efficiency because of the constant number of vertical layers over the whole of the computational field.

The FLOW module is the heart of Delft3D and is a multi-dimensional (2D or 3D) hydrodynamic (and transport) simulation program which calculates non-steady flow and transport phenomena resulting from tidal and meteorological forcing on a curvilinear, boundary fitted grid. Advection-diffusion solver is included to compute e.g. density gradients (due to non-uniform temperature and salinity concentration distributions) and pressure gradients terms in the momentum equation (density driven flows) are also done. Turbulence model to account for the vertical turbulent viscosity and diffusivity based on the eddy viscosity concept.

The FLOW module is coupled with other modules for example WAQ and ECO modules. The WAQ module simulates biochemical reactions like the decay of BOD and nitrification, growth of algae (primary production) and nutrient cycling, and exchange 
of substances with the atmosphere (oxygen, volatile organic substances, temperature) etc.

The ECO module of Delft3D simulates the bio (chemical) and biological processes related to growth of algae and nutrient dynamics which are relevant to the study and understanding of eutrophication phenomena. The module uses a different and more detailed set of processes than those available in the WAQ module. (from Delft Hydraulics webpage: http://delftsoftware.wldelft.nl/)

\section{0) MIKE 3D}

MIKE 3D is a professional engineering software package for 3D free-surface flows. It is applicable to simulations of flows, cohesive sediments, water quality and ecology in rivers, lakes, estuaries, bays, coastal areas and seas. MIKE 3 forms a unique professional software tool for consultancy services related to design, operation and maintenance tasks within the aquatic environment.

MIKE 3D simulates unsteady flow taking into account density variations, bathymetry and external forcing such as meteorology, tidal elevations, currents and other hydrographic conditions on staggered grids or flexile meshes with a vertical sigma coordinate approach. The MIKE 3D modelling system is based on the numerical solution of the three-dimensional incompressible Reynolds averaged Navier-Stokes equations subject to the assumptions of Boussinesq and (1) using a non-hydrostatic pressure formulation or (2) using a hydrostatic pressure assumption of hydrostatic pressure. The model consists of continuity, momentum, temperature, salinity and density equations and it is closed by a turbulent closure scheme. The density does not depend on the pressure, but only on the temperature and the salinity.

MIKE 3D Modules for Environmental hydraulics and Ecology include: AdvectionDispersion (AD) or Transport module (TR), Spill Analysis Module (SA), and ECO Lab. $\mathrm{AD}$ simulates the transport, dispersion and decay of dissolved or suspended substances. Typically used in cooling water and sewage outfall studies. SA (Spill Analysis) simulates the spreading and weathering of suspended substances and is used for only forecasting oil spill, evaluating spill scenarios for contingency plans, etc. ECO Lab is a complete numerical laboratory for ecological modelling. Physical, chemical and biological processes relevant to environmental problems and water pollution can be integrated and simulated with ECO Lab when combined with one of DHI's flow models for 1D, 2D or 3D flows. (from DHI webpage: http://www.dhigroup.com/) 


\section{APPENDIX II}

\section{Holt-Winters Forecasting Method}

Holt-Winters Forecasting Method is a combined function of Winters' method of exponential smoothing and Holt's method of a possible liner which exhibits both a trend and a seasonal pattern. The function of exponential smoothing is assigning geometrically declining weights to the data in time series so that more recent observations are given more weight than older observations. Therefore Holt-Winters Forecasting Method calculates dynamic estimates for three components: level, trend, and seasonal. The following method was cited from the user guidance of The Office for National Statistics (ONS) which is the executive office of the UK Statistics Authority and Chatfield and Yar (1988).

\section{1) Single Exponential Smoothing}

This is the simplest form of exponential smoothing and can be used only for data without any systematic trend or seasonal components. Given such a time series, a sensible approach is to take a weighted average of past values. So for a series, $y_{1}, y_{2}, \ldots$, $y_{n}$, the estimate of the value $(\hat{y})$ of $y_{n+1}$, given the information available up to time $n$, is:

$$
\hat{y}_{n+1 \mid n}=\sum_{i=0}^{\infty} w_{i} y_{n-i}
$$

in which $w_{i}$ is the weights given to the past values of the data in time series and sum to one. Since the most recent observations of the data series are also the most relevant, it is logical that they should be given more weight at the expense of observations further in the past. This is achieved by assigning geometrically declining weights to the data in time series. This decreases by a constant ratio and are of the form:

$$
w_{i}=a(1-a)^{i}
$$

in which $i=0,1,2, \ldots$ and $a$ is the smoothing constant in the range $0<a<1$. For all values of $a$ in the above equation will sum to $\sim 1$, the equation for the estimate of $y_{n+1}$ then becomes:

$$
\hat{y}_{n+1 \mid n}=\alpha y_{n}+\alpha(1-\alpha) y_{n-1}+\alpha(1-\alpha)^{2} y_{n-2}+\ldots \ldots
$$

Since

$$
\hat{y}_{n+1 \mid n}=\alpha y_{n}+(1-\alpha)\left(\alpha y_{n-1}+\alpha(1-\alpha) y_{n-2}+\ldots \ldots .\right.
$$

it can be seen that:

$$
\hat{y}_{n+1 \mid n}=\alpha y_{n}+(1-\alpha) \hat{y}_{n \mid n-1}
$$

in which $\hat{y}_{n \mid n-1}$ is simply the previous estimate.

This equation defines exponential smoothing and is extremely useful since we can now update each forecast using only two pieces of data: the latest observation; and the 
previous forecast. It can also be written in the alternative error correction form as follows:

$$
\hat{y}_{n+1 \mid n}=\alpha \varepsilon_{n}+\hat{y}_{n \mid n-1}
$$

in which $\varepsilon_{n}=y_{n}+\hat{y}_{n \mid n-1}$ and is the one step ahead prediction error.

The final matter with which to deal is the value of $a$, the smoothing constant. This will depend on the characteristics of the particular time series, especially the irregularity of the data. A high value of $a$ will lead to the majority of the weight being placed on the most recent observations whereas a low value of $a$ will mean that observations further in the past will gain more importance. A value of $a=1$ will obviously make the forecast equal to the final value of the series. The smoothing constant is commonly set between 0.05 and 0.3 , although it is possible to estimate $a$ by minimising the sum of squared prediction errors. This can be done by calculating $\sum \varepsilon_{t}^{2}$ for values of $a$ between 0 and 1 (say in steps of 0.1 ), where $\varepsilon_{t}$ is the one step ahead prediction error as stated above. By doing this it is possible to obtain, to the nearest tenth, the value of $a$ which minimises the sum of squared errors. Steps of 0.01 can then be taken around this value to minimise the sum of squares further. Accuracy at this point is not particularly important as the sum of squares function tends to be quite flat near the minimum.

\section{2) Holt's Method}

In single exponential smoothing, the forecast function is simply the latest estimate of the level. If a slope component is now added which itself is updated by exponential smoothing, the trend can be taken into account.

For a series $y_{1}, y_{2}, \ldots, y_{n}$, the forecast function, which gives an estimate of the series $l$ steps ahead can be written as:

$$
\hat{y}_{n+l \mid n}=m_{n}+l b_{n} \quad l=1,2, \ldots
$$

In which $m_{n}$ is the current level and $b_{n}$ is the current slope. Therefore, the one step ahead prediction is simply given by:

$$
\hat{y}_{t \mid t-1}=m_{t-1}+b_{t-1}
$$

Since there are now two terms to the exponential smoothing, two separate smoothing constants are required, $a_{0}$ for the level and $a_{1}$ for the slope. As in single exponential smoothing, the updated estimate of the level $m_{t}$ is a linear combination of $\hat{y}_{t \mid t-1}$ and $y_{t}$ :

$$
m_{t}=\alpha_{0} y_{t}+\left(1-\alpha_{0}\right)\left(m_{t-1}+b_{t-1}\right) \quad 0<\alpha<1
$$

This provides the level at time, $t$. Since the level at time $t-1$ is already known, it is possible to update the estimate of the slope: 


$$
b_{t}=\alpha_{1}\left(m_{t}-m_{t-1}\right)+\left(1-\alpha_{1}\right) b_{t-1}
$$

These equations can also be written in the appropriate error correction form:

$$
\begin{aligned}
& m_{t}=m_{t-1}+b_{t-1}+\alpha_{0} \varepsilon_{t} \\
& b_{t}=b_{t-1}+\alpha_{0} \alpha_{1} \varepsilon_{t}
\end{aligned}
$$

This method, known as Holt's method, requires starting values for $m_{t}$ and $b_{t}$ to be inputted, and estimates of the values for $a_{0}$ and $a_{1}$ to be made. It would be typical to find $0.02<a_{0}, a_{1}<0.2$, but they can be estimated by minimising the sum of squared errors as in single exponential smoothing. Also, it is often found that $m_{1}=y_{1}$, and $b_{1}=y_{2}-y_{1}$ are reasonable starting values.

\section{3) Holt-Winters Forecasting}

Holt's method can be extended to deal with time series which contain both trend and seasonal variations. The Holt-Winters method has two versions, additive and multiplicative, the use of which depends on the characteristics of the particular time series. The latter will be considered first.

The general forecast function for the multiplicative Holt-Winters method is:

$$
\hat{y}_{n+l \mid n}=\left(m_{n}+l b_{n}\right) c_{n-s+l} \quad l=1,2, \ldots
$$

where $m_{n}$ is the component of level, $b_{n}$ is the component of the slope, and $c_{n-s+l}$ is the relevant seasonal component, with $s$ signifying the seasonal period (e.g. 4 for quarterly data and 12 for monthly data.)

Therefore if a monthly time series is considered, the one step ahead forecast is given by:

$$
\hat{y}_{n+l \mid n}=\left(m_{n}+b_{n}\right) c_{n-11}
$$

The updating formulae for the three components will each require a smoothing constant. If once again $a_{0}$ is used as the parameter for the level and $a_{1}$ for the slope, and a third constant $a_{2}$, is added as the smoothing constant for the seasonal factor, the updating equations will be:

$$
\begin{aligned}
& m_{t}=\alpha_{0} \frac{y_{t}}{c_{t-s}}+\left(1-\alpha_{0}\right)\left(m_{t-1}+b_{t-1}\right) \\
& b_{t}=\alpha_{1}\left(m_{t}-m_{t-1}\right)+\left(1-\alpha_{1}\right) b_{t-1} \\
& c_{t}=\alpha_{2} \frac{y_{t}}{m_{t}}+\left(1-\alpha_{2}\right) c_{t-s}
\end{aligned}
$$


Once again, $a_{0}, a_{1}$, and $a_{2}$ all lie between zero and one. If the aforementioned additive version of Holt-Winters was used, the seasonal factor is simply added as opposed to multiplied into the one step ahead forecast function, thus:

$$
\hat{y}_{n+l \mid n}=m_{n}+b_{n}+c_{n-11}
$$

and the level and seasonal updating equations involve differences as opposed to ratios:

$$
\begin{aligned}
& m_{t}=\alpha_{0}\left(y_{t}-c_{t-s}\right)+\left(1-\alpha_{0}\right)\left(m_{t-1}+b_{t-1}\right) \\
& c_{t}=\alpha_{2}\left(y_{t}-m_{t}\right)+\left(1-\alpha_{2}\right) c_{t-s}
\end{aligned}
$$

The slope component, $b_{t}$, remains unchanged. The choice of starting values and smoothing parameters is of some importance and Chatfield and Yar (1988) discuss this in some depth. For starting values, it seems sensible to set the level component $m_{0}$, equal to the average observation in the first year, i.e.

$$
m_{0}=\sum_{t=1}^{s} y_{t} / s
$$

where $s$ is the number of seasons. The starting value for the slope component can be taken from the average difference per time period between the first and second year averages. That is:

$$
b_{0}=\frac{\left\{\sum_{t=1}^{s} y_{t} / s\right\}-\left\{\sum_{t=s+1}^{2 s} y_{t} / s\right\}}{s}
$$

Finally, the seasonal index starting value can be calculated after allowing for a trend adjustment, as follows:

$$
\begin{aligned}
& c_{0}=\frac{\left\{y_{k}-(k-1) b_{0} / 2\right\}}{m_{0}} \\
& c_{0}=y_{k}-\left\{m_{0}-(k-1) b_{0} / 2\right\}
\end{aligned}
$$

where $k=1,2, \ldots, s$. Obviously this will lead to $s$ separate values for $c_{0}$, which is what is required to gain the initial seasonal pattern.

The smoothing parameters are often selected between 0.02 and 0.2 . It is again possible to estimate them by minimising the sum of the squared one-step-ahead errors, but there is no exclusive combination of $a_{0}, a_{1}$, and $a_{2}$ which will minimise the square errors for all $t$.

\section{4) Correction for Autocorrelation of Residuals}


When the correction for autocorrelation of residuals is included, the Holt-Winters forecast function then becomes:

$$
\begin{array}{ll}
\hat{y}_{n+l \mid n}=\left(m_{n}+l b_{n}\right) c_{n-s+l}+r_{1}^{l} \varepsilon_{n} & \text { (multiplicative) } \\
\hat{y}_{n+l \mid n}=m_{n}+b_{n}+c_{n-s+l}+r_{1}^{l} \varepsilon_{n} & \text { (additive) }
\end{array}
$$

$\varepsilon_{n}$ is the one step ahead forecast error and $r_{1}$ is the first order autocorrelation coefficient of the forecast errors, given by:

$$
r_{1}=\frac{\sum \varepsilon_{n} \varepsilon_{n-1}}{\sum \varepsilon_{n}^{2}}
$$





\section{References}

Abdi, H. (2003). Partial Least Squares (PLS) Regression. In Lewis-Beck M., Bryman, A., Futing T. (Eds.), Encyclopedia of Social Sciences Research Methods. Thousand Oaks (CA).

Addy, K. and Green. L. (1996). Algae in Aquatic Ecosystems. University of Rhode Island, College of Resource Development Department of Natural Resources Science, Cooperative Extension. Fact Sheet No.96-4.

Akiyama, Stephan, J. and Stefan, H.G. (1987). Onset of Underflow in Slightly Diverging Channels $J$. Hydr. Engrg., Am. Soc. Civ. Engrs, 113 (7), pp. 825-844.

Alippi, C. and Braione, P. (2005). Classification Methods and Inductive Learning Rules: What We May Learn from Theory. IEEE Transactions On Systems, Man And Cybernetics-Part C: Aoolications and Reviews, Vol. No. 0, XXXXXX 0000.

Allaby, M. (1996). Basics of Environmental Science. Routledge, London.

Alvelius, K. (1999). Studies of Turbulence and its modelling through Large Eddy-and Direct Numerical Simulation. Department of Mechanics. Stockholm, Sweden, Royal Institute of Technology.

Ambrose, R.B., Wool, T., Connolly, J.P., and Schanz, R.W. (1988). WASP4, a hydrodynamic and water quality model: Model theory, user's manual, and programmer's guide. Envir. Res. Lab., US EPA 600/3-87/039, Athens, GA.

Ambrosetti, W., Barbanti, L. and Sala, N. (2003). Residence time and physical processes in lakes. $J$. Limnol., 62(Suppl.), pp. 1-15.

APHA, AWWA and WPCF. (1995). Standard methods for the examination of water and wastewater, 19th ed.

Arega, F., and Lee, J.H.W. (2000). Long-term circulation and eutrophication model for Tolo Harbour, Hong Kong. Water Qual. Ecosyst. Model., 1:1-4, 169-192.

Armstrong, J.S. (2005). Findings from Evidence-based Forecasting: Methods for Reducing Forecast Error. International Journal of Forecasting. January 252005.

Baffi, G., Martin, E.B., and Morris, A.J. (1999). Non-linear projection to latent structures revisited (the neural network PLS algorithm). Computers and Chemical Engineering 23, pp. 1293-1307.

Bakker, A. (2006). Lecture 9 - Kolmogorov's Theory in Applied Computational Fluid Dynamics. http://www.bakker.org

Baruah, P.J., Tamura, M., Oki, K. and Nishimura, H. (2001). Neural Network Modelling Of Lake Surface Chlorophyll And Sediment Content From Landsat TM Imagery. Centre for Remoute Imaging, Sensing and Processing (CRISP), National University of Singapore; Singapore Institute of Surveyprs and Valuers (SISV); Asian Association on Remoute Sensing (AARS).

Basson, G. (1998). Prediction of Sediment Induced Density Current Formation in Reservoirs. 3rd International Conference on Hydro-Science and -Engineering. Brandenburg University of Technology at Cottbus/Berlin, Germany, August 31 - September 31998.

Beck, A. and Koop, H.U. (2001). Analysis of the photobiont population in lichens using a single-cell manipulator. Symbiosis 31: 57-67.

Benjamin, M.M. (2002). Water chemistry. McGraw-Hill: Boston.

Berdalet, E., Estrada, M. and Marrasé, C. (2005). Living below the Kolmogorov length scale. Or why phytoplankton cells care about small scale turbulence. Geophysical Research Abstracts, Vol.7, 09943.

Bezdek, J.C. (1981). Pattern Recognition with Fuzzy Objective Functions. New York: Plenum.

Bhattacharya, B., Solomatine, D.P. (2005). Neural networks and M5 model trees in modelling water level discharge relationship, Neurocomputing, Vol. 63, pp. 381-396 
Bhattacharya, B., Solomatine, D.P. (2006). Machine learning in sedimentation modeling, Neural Networks, Vol. 19, pp. 208-214.

Birge, E.A. (1897). Plankton studies on Lake Mendota, 2. The crustacea from the plankton from July, 1894, to December, 1896. Transactions of the Wisconsin Academy of Science, Arts and Letters 11, pp. 274448.

Boris, J.P. and Book, D.L. (1973). Flux-Corrected Transport. I. SHASTA, A Fluid Transport Algorithm That Works, J. Comp. Phys., Vol. 11, pp. 38-69, 1973.

Borsuk M., Higdon D., Stow C. and Reckhow K. (2001). A Bayesian hierarchical model to predict benthic oxygen demand from organic matter loading in estuaries and coastal zones. Ecological Modelling 143, pp. 165-181.

Bowie, G.L., Mills, W.B., Porcella, D.B., Campbell, C.L., Pagenkopf, J.R., Rupp, G.L., Johnson, K.M., Chan, P.W.H., and Gherini, S.A. (1985). Rates, constants, and kinetics formulations in surface water quality modelling. Report EPA/600/3-85/040, 2nd ed, Environmental Research Laboratory, U.S. Environmental Protection Agency, Athens, GA.

Buras, N. (2001). Editorial, Water Resources-Unresolved Issues. Journal of Water Wesources Planning and Management / November/December 2001, p. 353.

Burge. K and Breen, P.F (2006). Detention time design criteria to reduce the risk of excessive algal growth in constructed water bodies. Proceedings for the $4^{\text {th }}$ International Conference on Water Sensitive Urban Design. Melbourne, 2-6 April 2006.

Canfield, D.E. Jr., and Bachmann, R.W. (1981). Prediction of total phosphorus concentrations, chlorophyll a, and Secchi depths in natural and artificial lakes. Can. J. Fish. Aquat. Sci. 38, pp. 414423.

Carofano, G.C. (1984). Blast Computation Using Harten's Total Variation Dinfinishing Scheme, Technical Report ARLCB-TR-84029

Chwang, A.T., Joseph, H.W., Lee, D.Y. and Leung, C. Eds. (1996). Hydrodynamics: Theory and Applications. Volume 1. Taylor \& Francis.

Chapra, S. and Canale, R. (1988). Numerical Methods for Engineers. McGraw-Hill.

Chapra, S.C., and Reckhow, K.H. (1983). Engineering approaches for lake management, Vol. 2, Mechanistic modeling, Ann Arbor Science, Ann Arbor, Mich.

Chapra, S.C. (1997), Surface Water-Quality Modeling. McGRAW-HILL International Editions Civil Engineering Series. ISBN 0-07-011364-5.

Chatfield, C. and Yar, M. (1988). Holt-Winters forecasting: some practical issues. The Statistician. 37, pp. 129-140.

Cohen, J. (1988). Statistical power analysis for the behavioural sciences $\left(2^{\text {nd }}\right.$ Ed). Hillsdale, NJ: Lawrence Erlbaum Associates.

Cole, T.M. and Wells, S.A. (2003). Instruction Report EL-03-1. CE-QUAL-W2: A Two-Dimensional, Laterally Averaged, Hydrodynamic and Water Quality Model. Version 3.2. User Manual. U.S. Army Corps of Engineers, Washington, DC 20314-1000.

Cole, T.M. and Buchak, E.M. (1995). CE-QUAL-W2: A two-dimensional, laterally averaged hydrodynamic and water quality model, version 2.0, User Manual. Instruction Report El-95, Waterways Experiment Station, Vicksburg, MS.

Connolly, J.P. and R. Winfield. (1984). A User's Guide for WASTOX, a Framework for Modeling the Fate of Contaminants in Aquatic Environments. Part 1: Exposure Concentration. U.S. Environmental Protection Agency, Gulf Breeze Laboratory, Gulf Breeze, FL. EPA-600/3-84-077.

Cordier, T. (2006). Lessons Learned From the Model-Driven Architecture Applied to Critical Systems Reliability: A Case Study. Department of Computer and Systems Sciences, Royal Institute of 
Technology / Stockholm University

Correll, D.L. (1998). The Role of Phosphorus in the Eutrophication of Receiving Waters: A Review. J. Environ. Qual. 27, pp. 261-266.

Cowen, W.F. and Lee, G.F. (1976). Algal nutrient availability and limitation in Lake Ontario during IFGYL. Part 1. Office of Research and Development, U.S. Environmental Protection Agency, Duluth, MN, EPA-600/3-76-094a:218.

Cushman-Roisin, B. (1994). Introduction to geophysical fluid dynamics. Prentice Hall, 320pp

Deissler, R.G. (1992), Turbulent Fluid Motion IV Averages, Reynolds Decomposition, and the Closure Problem. National Aeronautics and Space Administration, Lewis Research Center, Cleveland, Ohio 44135

D'Elia, C.F. and DeBoer, J.A. (1978). Nutritionl studies of two red algae: II. Kinetics of ammonium and nitrate uptake. J. Phycol. 14, pp. 266-272.

del Giorgio, P. and Cole, J.J. (1998). Bacterial growth efficiency in natural aquatic systems. Annu Rev Ecol Syst 29, pp. 503-541.

de la Fuente, A. and Niño Y. (2008). Pseudo 2D ecosystem model for dendritic reservoir. Ecological Modelling 213, pp.389-401.

Despic, O. and Simonovic, S. (1997). Methods in evaluating qualitative criteria in water resources multicriteria decision-making. Water Resources Research Report no. 37, Dept. of Civil and Geological Engineering, University of Manitoba.

Di Toro, D.M., Fitzpatrick, J.J. and Thomann, R.V. (1983). Water quality analysis simulation program (WASP) and model verification program (MVP) documentation. Hydroscience Inc., Westwood, NY.

Duan, Y, Smith, E.P. and Ye, K. (2006). Using Power Priors to Improve the Binomial Test of Water Quality. Journal of Agricultural, Biological, and Environmental Statistics. Jun, Vol. 11, No. 2: 151168

Edinger, J.E., Buchak, E.M., Merritt, D.H. (1983). Longitudinal vertical hydrodynamics and transport with chemical equilibria for Lake Powell and Lake Mead. In: French RH ed. Salinity in Watercourses and Reservoirs. Stoheham, MA, United States, Butterworth Publishers, pp. 213-222.

Elias, R.J., Montgomery, D.C. and Kulahci, M. (2006). An overview of short-term statistical forecasting methods. International Journal of Management Science and Engineering Management Vol. 1, No. 1, pp. 17-36

Falkoeski, P.G. (2000). Rtionalizing Elemental Ratios in Unicellular lgae. J. Phycol. 36, 3-6.

Fan, J. (1960). Experimental Studies on Density Currents. Scientia Sinica, 9(2), pp. 275 - 303.

Fischer, H.B., List, E.G., Koh, R.C.Y., Imberger, J. and Brooks, N.H. (1979), Mixing in Inland and Coastal Waters. Academic Press, New York, NY.

Fitzgerald, G.P. and Nelson, T.G. (1966). Extractive and enzymatic analyses for limiting or surplus phosphorus in algae, J. Phycol. 2, pp. 32-37.

Findikakis A.N. and Law. A.W.K. (1999). Wind Mixing In Temperature Simulations for Lakes and Reservoirs. Journal of environmental Engineering, May 1999, pp. 420-428.

Ford, D.E. (1990). Reservoir transport process. In:Thornton, K.W. et al. (ed.): Reservoir Limnology: ecological perspectives. John Wiley \& Sons, New York, pp. 15-42.

Forsberg, C., Ryding, S.O., Claesson, A. and Forsberg, A. (1978). Water chemical analysis and/or algal assay-Sewage effluent and polluted lake water studies. Mitt. Int. Ver. Limnol. 21, pp. 353-263.

Fox, N. (2008). A guide to content of a documentary procedure to meet the Quality Assurance requirements of CEOS. CEOS Guide: QA4EO-CEOS-GEN-DQK-002.

Friedman, J. H (1991). Multivariate Adaptive Regression Splines (with discussion). Annals of Statistics 
19, 1. (software).

Fjelde, K..K and Karlsen, K.H. (2002). High-resolution hybrid primitive-conservative upwind schemes for the drift flux model. Computers and Fluids 31, 335-367

Gaugush, R.F. (1986). Statistical methods for reservoir water quality investigations. Instruction Report E86-2. U.S. Army Engineer Waterways Experiment Station. Vicksburg, MS.

Gelda R.K. and Effler, S.W. (2007). Testing and application of a two-dimensional hydrothermal model for a water supply reservoir: implications of sedimentation. Journal of Environmental Engineering Science 6, pp. 73-84.

Gerber, N. and Lechevalia, H. (1965). Geosmin and earthy smelling isolated from actinomycetes. Applied Microbiology, 13, 935.

Gerhold, R.M. (1976) Algal nutritional bioassay of Lake Wylie, North Carolina. In: Biostimulation and Nutrient Assessment (edited by E. J. Middlebrooks, D. H. Falkenborg and T. E. Maloney), 175-220. Utah State University, Logan, Utah.

Giammatteo, P.A., Schindler, J.E., Waldron, M.C., Freedman, M.L., Speziale, B.J., and Zimmerman, M.J. (1983). Use of Equilibrium Programs in Predicting Phosphorus Availability. Intl. Symp. Environ. Biogeochem. Stockholm, Sweden.

Glibert, P.M. (2007). Eutrophication and Harmful Algal Blooms: A Complex Global Issue, Examples from the Arabian Seas including Kuwait Bay, and an Introduction to the Global Ecology and Oceanography of Harmful Algal Blooms (GEOHAB) Programme. International Journal of Oceans and Oceanography, ISSN 0973-2667 Volume 2 Number 1 (2007), pp. 157-169.

Gode, P. and Overbeck, J. (1972). Untersuchungen zur heterotrophen Nitrifikation im See. Z. allg. Mikrobiol. 12, 567-574 (1972)

Goldman, C.R. and Horne, A.J. (1983). Limnology. McGraw-Hill Int. Book Comp. 464 pp. Auckland.

Gordon, J.A. (1980). An Evaluation of the LARM Two-Dimensional Model for Water Quality Management Purposes. Proceedings of the Symposium on Surface-Water Impoundments, Minneapolis, MN, 2-5 June 1980, pp. 518-527.

Gordon, J.A. (1981). LARM Two-Dimensional Model: An Evaluation. ASCE J. of the Environmental Engineering Division 107, pp. 877-886.

Gordon, J.A. (1983). Short-Term Hydrodynamics in a Stratified Reservoir. Environmental Engineering, Proceedings of the ASCE Specialty Conference, Hilton Harvest House, Boulder, Colo., July 6-8 1983, pp. 524-531.

Zhuravlev, Y.N. and Avetisov, V.A. (2006). The definition of life in the context of its origin. Biogeosciences, 3, 281-291.

Greene, J.C., Miller, W.E., Shiroyama, T. and Maloney, T.E. (1975). Utilization of algal assays to assess the effects of municipal, industrial, and agricultural wastewater effluents upon phytoplankton production in the Snake River system. Water, Air, Soil Pollut. 4, pp. 415-434.

Greene, J.C., Soltero, R.A., Miller, W.E., Gasperino, A.F. and Shiroyama, T. (1976) The relationship of laboratory algal assays to measurements of indigenous phytoplankton in Long Lake, Washington. Biostimulation and Nutrient Assessment, pp. 93-126. Utah State University, Logan, Utah

Guinot, V. and Gourbesville, P. (2003). Calibration of physically based models: back to basics? Journal of Hydroinformatics 05. 4. 2003. pp 233 244.

Haeckel, E. (1866). Generelle Morphologie der Organismen. Berlin G. Reimer.

Harten, A., Osher, S., Engquist, B. and Chakravarthy, S.R. (1987). Uniformly high order accurate essentially non-oscillatory schemes III, Journal of Computational Physics 71, pp. 231-303.

He, G.W., Wang, M. and Lele, S.K. (2002). Evaluation of subgrid-scale models in terms of time 
correlations. Center for Turbulence Research Proceedings of the Summer Program 2002

Heathcote, I.W. (1998), Integrated Watershed Management: Principles and Practice. John Wiley \& Sons, Inc. ISBN0-471-18338-5.

Heiskary, S. and Markus, H. (2001). Establishing Relationships among Nutrient Concentrations, Phytoplankton Abundance, and Biochemical Oxygen Demand in Minnesota, USA, Rivers. Lake and Reservoir Management 17 (4): 251-262.

Hodges, B.R., Imberger, J. and Laval, B. and Appt, J. (2000). Modeling the Hydrodynamics of Stratified Lakes. Hydroinformatics 2000. Iowa Institute of Hydraulic Research, 23-27 July 2000.

Hofmann, A. and Filella, M. (1999). Transport of Suspended Matter in the Hypolimnion of Lake Lugano: a Comparison of Field Observations and Model Predictions. J. Great Lakes Res. 25(4):865-882.

Howarth, R.W. and Marino,R. (2006). Nitrogen as the limiting nutrient for eutrophication in coastal marine ecosystems: Evolving views over three decades. Limnol. Oceanogr., 51(1, part 2), 2006, 364 376

Hur, J., Jung, N.C. and Shin, J.K. (2007). Spectroscopic Distribution of Dissolved Organic Matter in a Dam Reservoir Impacted by Turbid Storm Runoff. Environ Monit Assess 133, pp.53-67

Hutchinson, G.E. (1961). Hutchinson, The paradox of the plankton, Am. Nat. 95 , pp. 137-145.

IARC. (1992). IARC Monographs on the Evaluation of Carcinogenic Risks to Humans. Vol 52: Chlorinated Drinking-Water; Chlorination By-products; Some other Halogenated Compounds; Cobalt and Cobalt Compounds. Lyon: International Agency for Research on Cancer.

ILEC. (2005). Managing Lakes and their Basins for Sustainable Use: A Report for Lake Basin Managers and Stakeholders. International Lake Environment Committee Foundation, Kusatsu, Japan. (Available online at http://www.ilec.or.jp)

Imberger, J., and Patterson, C.J. (1981), A Dynamic Reservoir Simulation Model - DYRESM: 5. In Fisher, B.H. (Ed.) Transport Models for Inland and Coastal Waters. Berkeley, California (USA), Academic Press.

Imboden, D. M. and Wüest, A. (1995), Mixing mechanisms in lakes, in Lerman, A., Imboden, D.M. and Gat, J. R. eds, 'Physics and Chemistry of Lakes', Springer Verlag, pp. 83 138.

Ishige, T., Krause, M., Bott, M., Wendisch, V.F. and Sahm, H. (2003). The Phosphate Starvation Stimulon of Corynebacterium glutamicum Determined by DNA Microarray Analyses. Journal of Bacteriology, Aug. 2003, p. 4519-4529.

Ivey, G., Winters, K. and DeSilva, I. (1999)“"Turbulent mixing in an internal wave energized benthic boundary layer," submitted to J. Fluid Mech.

James, D.H. and Lee, G.F. (1974). A Model of Inorganic Carbon Limitation in Natural waters. Water, Air, and Soil Pollution 3 (1974) 315-320.

Jeong, K.S., Kim, D.K. and Jo, G.J. (2006). River phytoplankton prediction model by Artificial Neural Network: Model performance and selection of input variables to predict time-series phytoplankton proliferations in a regulated river system. Ecological Informatics 1, pp. 235-245.

Johnson, B. (1981). A review of numerical reservoir hydrodynamic modelling," Technical Report E-81-2, Hydraulics Laboratory, Corps of Engineers., Waterways Experiments Stations, Vicksburg, MS.

Jørgensen, S.E. (1994). Review and comparison of goal function in system ecology. Vie Milieu 44, pp. $11-20$.

Jørgensen, S.E. (Ed.). (1997). Integration of Ecosystem Theories: A Pattern, 2nd ed. Kluwer Academic Publishers, Dordrecht, 388 p.

Jørgensen, S.E. and Vollenweider, R.A. (1988). Guidelines of Lake Management. In Principles of Lake Management. Kusastu, Japan, United Nations Environment Programme (UNEP). 
Jung, N.C. and Price R. K. (2005). Applicability of Data-Driven Modelling For Reservoir Water Quality Management. XXXI IAHR Congress, Vol. 1, pp 174-175.

Juntasaro, V. and Marquis, A.J. (2004). Comparative Study of Flux-limiters Based on MUST Differencing Scheme. International Journal of Computational Fluid Dynamics, Vol. 18 (7), pp. 569 576.

Kaczmarek. (2004). Integrated Watershed Management, Ecohydrology \& Phytotechnology, Manual, Chapter 3B. The United Nations Environment Programme International Environmental Technology Centre 2-110 Ryokuchi Koen, Tsurumi-ku, Osaka 538-0036 1091 Oroshimo-cho, Kusatsu-City, Shiga 525-0001, Japan

Karimipour, F., Delavar, M.R. and Rezayan, H. (2005). Neighborhood Analysis in Water Pollution Estimation. International Society for Environmental Information Sciences, Environmental Informatics Archives, Vol. 3, pp. 232-238.

Kawamura, K. and Kaplan, I.R. (1986). Biogenic and Anthropogenic Organic Compounds in Rain and Snow Samples Collected in Southren California. Atmospheric Environment Vol. 20, No. 1, pp. 115124.

Kelderman, P. (1985). Nutrient dynamics in the sediment of lake Grevelingen (SW Netherlands).

Kennedy, R.K. and Walker, W.W. (1990). Reservoir Nutrient Dynamics. Reservoir Limnology: Ecological Perspectives, pp109. A Wiley-Interscience Publication John Wiley \& Sons, Inc.

Kilham, S.S. (1978). Nutrient kinetics of freshwater planktonic algae using batch and semicontinuous methods. Mitt. Int. Ver. Theor. Angew. Limnol. 21, p. 147-157.

Kim, B.R., Higgins, J.M., and Bruggink, D.J. (1983). Reservoir Circulation Patterns and Water Quality. J. Envir. Engrg. 109, pp. 1284-1294.

Kim, B., Park, J., Hwang, G., Jun, M., Choi, K. (2001) Eutrophication of Reservoirs in South Korea. The Japanese Society of Limnology.

Kim, B., Choi, K., Kim, C., Lee, U., and Kim, Y. (2000). Effects of the summer monsoon on the distribution and loading of organic carbon in a deep reservoir, Lake Soyang, Korea. Wat. Res. Vol. 34, No. 14, pp. 3495-3504.

Kim, B., Sa, S.H., Kim, M., Lee, Y., and Kim, J.K. (2007). The Limiting Nutrient of Eutrophication in Reservoirs of Korea and the Suggestion of a Reinforced Phosphorus Standard for Sewage Treatment Effluent. Journal of Korean Society on Water Quality, Vol. 23, No. 4, pp. 512-517

Kim, Y., Lee,Y., Gee, C.S. and Choi, E. (1997). Treatment of Taste and Odor Causing Substances in Drinking Water. Wat. Sci. Tech 35, No.4, pp. 512-517

Kimmel, B.L. and Groeger, A.W. (1984). Factors controlling primary production in lakes and reservoirs; A perspective. pp. 277-281. In Lake and Reservoir Management. U.S. EPA-440/5-84-001.

Kleeberg A. and Kozerski, H.-P. (1997): Phosphorus release in Lake Großer Müggelsee and its implications for lake restoration. Hydrobiologia 242/243: 9-26.

K MOE (Korea Ministry of Environment). (1990). Water quality standards.

Kolmogorov, A.N. (1941). The Local Structure of Turbulence in Incompressible Viscous Fluid for Very Large Reynolds Numbers. C. R. Acad. Sci. USSR 30, 301.

Kolmogorov, A.N. (1942): Sur l'estimation statistique des paramètres de la loi de Gauss. (Russian. French Summary), Bull. Acad. Sci. URSS. Sér. Math. [Izvestia Akad. Nauk SSSR] 6, 1/2, pp. 3-32.

Kompare, B., Steinman, F., Cerar, U. and Dzeroski, S. (1997). Prediction of rainfall runoff from catchment by intelligent data analysis with machine learning tools within the artificial intelligence tools. Acta Hydrotech. 16/17, pp. 79-94.

Kowalik, Z. and Murty, T.S. (1993). Numerical modeling of ocean dynamics. Singapore, World Scientific Publishing Co., 481 p. 
Kreinovich, Vladik., Berleant, Daniel J., Ferson, Scott., Lodwick, and Weldon A. Combining Interval, Probabilistic, and Fuzzy Uncertainty: Foundations, Algorithms, Challenges: An Overview. http://www.cs.utep.edu/vladik/2005/tr05-09a.pdf

Kuo, J., Lung, W., Yang, C., Liu, W., Yang, M., and Tang, T. (2006). Eutrophication modelling of reservoirs in Taiwan. Environmental Modelling \& Software 21, pp. 829-844

Kuzmin D. and Turek S. (2004). High-resolution FEM-TVD schemes based on a fully multidimensional flux limiter. Journal of Computational Physics, Volume 198, Issue 1, pp. 131-158.

K-water. (1991). Design Report of the Yongdam Reservoir.

K-water. (2002). Nutrient production and load in the Yongdam reservoir basin.

Lang D.S. and Brown E.J. (1981). Phosphorus-limited growth of a green alga and a blue-green alga. Appl. Environ. Microbiol. 42, pp. 1002-1009.

Langmuir, I., (1938). Surface motion of water induced by wind, Science 87, pp. 119-123.

Lapointe, B.E., Barile, P.J., Littler, M.M., Littler, D.S., Bedford, B.J., and Gasque, C. (2005). Macroalgal blooms on southeast Florida coral reefs I. Nutrient stoichiometry of the invasive green alga Codium isthmocladum in the wider Caribbean indicates nutrient enrichment. Harmful Algae 4, pp. 1092-1105.

Lee, J.J., McEnery, M.E., Röttger, R., and Reimer, C.W. (1980). The isolation, culture and identification of endosymbiotic diatoms from Heterostegina depressa D,Orbigny and Amphistegina lessonii D’Orbigny (Larger Foraminifera) from Hawaii. Botanica mar. 23, pp. 297-301.

Lee, S.J., Jang, M.H., Kim, H.S., Yoon, B.D. and Oh, H.M. (2000). Variation of microcystin content of Microcystis aeruginosa relative to medium N:P ratio and growth stage. Journal of Applied Microbiology 89, pp. 323-329.

Leggett, R.W. and Williams, L.R., (1981): A reliability index for models. Ecol. Model. 13, pp. 303-312.

Lévêque, E. (2005) An Introduction to Turbulence in Fluids and Modelling Aspects. EDP Sciences 1, 37 p.

Limno-Tech, Inc. 2002. Descriptive Inventory of Models with Prospective Relevance to Ecological Impacts of Water Withdrawals. Excellence in Environmental Solutions Since 1975. Ann Arbor, Michigan.

Lindsay, J. B., Shang, J. Q., and Rowe, R. 2002. Discussion of "Using Complex Permittivity and Artificial Neural Networks for Contaminant Prediction" Journal Of Environmental Engineering (C) ASCE / November 2003, pp. 1069-1071.

Liu, S., Meneveau, C., and Katz, J. (1995). Experimental Study of Similarity Subgrid-Scale Models of Turbulence in the Far-Field of a Jet. Applied Scientific Research 54: 177-190, 1995.

Liu, X.D., Osher, S. and Chan, T. (1994). Weighted essentially non-oscillatory schemes, Journal of Computational Physics, pp. 200-212.

López, E.L. and Dávalos-Lind, L. (1998). Algal growth potential and nutrient limitation in a tropical river-reservoir system of the Central Plateau, Mexico. Aquatic Ecosystem Health and Management 1, pp. 345-351.

MacQueen, J.B. (1967). Some Methods for classification and Analysis of Multivariate Observations, Proceedings of 5-th Berkeley Symposium on Mathematical Statistics and Probability, Berkeley, University of California Press, 1, pp. 281-297.

Marshall, E.M. and Bakker, A. (2003). Computational Fluid Mixing. Fluent Inc., 10 Cavendish Court, Lebanon, NH 03766, USA

Martin, J.L. (1988). Application of two-dimensional water quality model. Journal of Environmental Engineering, ASCE, 114, pp. 317-336. 
Martin, J.L. and McCutcheon, S.C. (1999). Hydrodynamics and Transport for Water Quality Modelling, Athens, GA, Lewis Publishers

MavIntyre, S. (1998). Turbulent Mixing and Resource Supply to Phytoplankton. In Imberger, J. (Ed.) Coastal and Estuarine Studies : Physical Processes in Lakes and Oceans. Broome, Western Australia, American Geophysical Union.

McKnight, D.M., Boyer, E.W., Westerhoff, P.K., Doran, P.T., Kulbe, T., and Andersen, D.T. (2001). Spectrofluorometric characterization of dissolved organic matter for indication of precursor organic material and aromaticity. Limnol. Oceanogr. 46, pp. 38-48.

Meybeck, M. and Helmer, R. (1996). Introduction. In: D. Chapman [Ed.] Water Quality Assessments. A Guide to the Use of Biota, Sediments and Water in Environmental Monitoring. 2nd edition. Chapman \& Hall, London.

Meybeck, M., Kimstach,V., and Helmer. (1992). Strategies for Water Quality Assessment. In Chapman, D. (Ed.) Water Quality Assessments: A guide to use of biota, sediments and water in environmental monitoring. UNESCO/WHO/UNEP.

MIKE3 Reference Manual. (2005). Hydrodynamic Module Scientific Documentation. DHI Software.

Miller, W.E., Maloney, T.E. and Greene, I.C. (1974). Algal productivity in 49 lake waters as determined by algal assays. Wat. Res. 8, pp. 667-679.

Mitchell, T.M. (1997). Machine Learning, International Edition, McGraw-Hill Book Co, Singapore, ISBN 0-07-042807-7

Moffatt \& Nichol Engineers, (2005). Hydrodynamic Modelling Tools and Techniques. In Conservancy, C. S. C. (Ed.) Oakland, California, Moffatt \& Nichol, Inc.

Montgomery, J.M. (1985). Water Treatment Principles and Design, Wiley, New York

Moody, J. and Darken, C. (1988), Learning with Localized Receptive Fields, In Proceedings of the 1988 Connectionist Models Summer School, D. Touretzky, G. Hinton, T. Sejnowski, (Eds.), pp. 133-143, Morgan-Kaufmann, San Mateo.

Moore, L. and Thornton, K. ed. (1988). The Lake and Reservoir Restoration Guidance Manual. First Edition. EPA 440/5-88-002, US EPA, Office of Wetlands, Oceans, and Watersheds, Assessment \& Watershed Protection Division, WH-553, 401 M Street, S.W., Washington, DC 20460.

Munk, W.H. and Anderson, E.R. (1948). Notes on the theory of the thermocline. J. Mar. Res. 1.

KNIER (Korean National Institute of Environmental Research). (2006). Hydraulic and Water Quality Modelling. Publication No. 11-1480523-000052-01.

Novotny, V. (1995). Nonpoint Pollution and Urban Stormwater Management.. Vol. 9. CRC Press.

Nürnberg, G.K. (1988). The prediction of phosphorus release rates from total and reductant-soluble phosphorus in anoxic lake sediments, Can. J. Fish. Aquat. Sci. 45: 453-462.

Nusch, E.A. (1980). Comparison of different methods for chlorophyll and phaeopigment determination. Arch. Hydrobiol. Beih. (Ergebn. Limnol.) 14, pp. 14-36.

Nyholm, N. and Lyngby, J.E. (1988). Algal Bioassays In Eutrophication Research-A Discussion In The Framework Of A Mathematical Analysis War. Res., Vol. 22, No. 10, pp. 1293-1300.

Odum, E.P. (1971). Fundamentals of ecology. 3rd ed. Saunders, Philadelphia and London.

Ostrovsky, I., Y.Z. Yacobi, P. Walline, and I. Kalikhman, "Seiche-induced mixing: Its impact on lake productivity," Limno. Oceanogr., 41, pp. 323-332, 1996.

Oran, Elaine S. and Boris, J.P. (2000). Numerical Simulation of Reactive Flow. Published by Cambridge University Press, 2000. ISBN 0521581753, 9780521581752.

Orkin, M. and Drogin, R. (1990). Vital Statistics. New York: McGraw Hill.

Overbeck, J. (1988). Ecosystem Concepts. In Guidelines of Lake Management, Vol. 1, Principles of Lake 
Management, S.E. Jorgensen and R.A. Vollenweider (Eds.). ILEC, UNEP.

Payne, A.G. (1976). Application of the algal assay procedure in biostimulation and toxicity testing. In: Bio stimulation and Nutrient Assessment (edited by Middlebrooks, E.J., Falkenborg D.H. and Maloney, T.E.), pp. 3-28. Utah State University, Logan, Utah.

Pentecost, A. (1999), Analysing Environmental Data. ISBN 058231058 X. Addison Wesley Longman Singapore (Pte) Ltd.

PHOSLOCK. (2008). Blue-Green Algae Management in Aquaculture. Phoslock Water Solutions Ltd. (http://www.phoslock.com.au/Blue-Green\%20Algae\%20Management $\% 20$ in\%20Aquaculture.pdf).

Piomelli, U. and Chasnov, J.R. (1995). Large Eddy Simulations: Theory and Applications. In Hallback, M., Hennington, D.S., Johannsson, A.V. and Alfredsson, P.H. (Ed.) Turbulence and Transition Modelling. Stockholm, Sweden, Kluwer Academic Publishers.

Popescu, I. (2004). Numerical methods for differential equations. Hydroinformics, UNESCO-IHE Delft.

Price, R. K. (2002). Hydroinformatics - Lecture1. Proceedings of RBM 2002: Advanced study course on river basin modeling for flood risk mitigation, 7-18 October 2002, The University of Birmingham, UK, pp5.1-5.19.

Quinlan, J.R. (1992). C4.5 Programs for Machine Learning. Morgan Kaufman Publishers Inc.

Raven, J.A. (1970). Exogenous inorganic carbon source in plant photosynthesis. Biol Rev 45, pp. 167-221.

Read, B.J. (1999) Data Mining and Science? Knowledge discovery in science as opposed to business. 12th ERCIM Workshop on Database Research. Amsterdam, 2-3 November. http://www.ercim.org/publication/ws-proceedings/12th-EDRG/index.html

Reckhow, K.H., Clements, J.T., and Dodd, R.C., 1990. Statistical evaluation of mechanistic water-quality models. J. Environ. Eng. 116(2), 250-268.

Recknagel, F., Bobbin, J., Whigham, P. and Wilson, H. (2002). Comparative application of artificial neural networks and genetic algorithms for multivariate time-series modelling of algal blooms in freshwater lakes. Journal of Hydroinrormatics/04.2/2002.

Redfield, A.C. (1934). On the proportions of organic derivatives in sea water and their relation to the composition of plankton. In Daniel, R.J. (Ed.) James Johnstone Memorial Volume. University Press of Liverpool, pp. 177-192.

Redfield, A,C. (1958). The biological control of chemical factors in the environment Amer. Scientist. 46, pp. 205-221.

Robertson, A. (1997). Limiting Nutrient Workshop 1997. LWRRDC Occasional Paper 7/99. School of Science and Technology, Charles Sturt University, Wagga NSW 2678.

Rodi, W. (1984) Turbulence Models and Their Application in Hydraulics, 2nd Edn. International Association for Hydraulics Research, Delft.

Røed, L. P. (2005). Challenges met in the development of integrated river basin models: quality assurance procedures. Norwegian Meteorological Institute. Note No. 4/2005, Oceanography, Oslo, 3rd February 2005.

Rood, E.P. (1996). Validation strategy for 'RANS': Computational ship hydraulics. Hydrodynamics, Chwang, Lee \& Leung (eds) (C) 1996 Balkema, Rotterdam.

Rosipal, R. and Krämer, N. (2006). Overview and Recent Advances in Partial Least Squares. Saunders, C. et al. (Eds.): SLSFS 2005, LNCS 3940, pp. 34-51. C Springer-Verlag Berlin Heidelberg.

Ross, T.J. (1995). Fuzzy Logic with Engineering Applications. McGraw Hill, New York.

Rump, H.H. and Krist, H. (1988). Laboratory manual for the examination of water, waste water and soil. VCH Pub. Comp. 190 pp.

Sanford, L.P. (1997). Turbulent mixing in experimental ecosystem studies. MARINE ECOLOGY 
PROGRESS ERIES Mar Ecol Prog Ser. Vol. 161: 265-293.

Sarle, W.S., (1994). Neural Networks and Statistical Models. Proceedings of the Nineteenth Annual SAS Users Group International Conference, April 1994. SAS Institute Inc., Cary, NC, USA.

Sas, H. (1989). Lakes restoration by reduction of nutrient loading: Expectations, experiences, extrapolations. Academia Verlag Richarz, 497 p.

Schalkoff, R.J. (1997). Artificial Neural Networks, McGraw-Hill Higher Education.

Schink, D.R. and Guinasso, N.L. Jr. (1977). Modelling the influence of bioturbation and other processes on carbonate dissolution at the sea floor. In: Andersen, N.R. and A. Malahoff, The Fate of Fossil Fuel $\mathrm{CO}_{2}$. in the Oceans, pp. 375-399.

Schladow, S.G. and Hamilton, D.P. (1996). Prediction of water quality in lakes and reservoirs. Part II Application to Prospect Reservoir. Ecol Modelling 96: pp111-123

Schnoor, J.L. (1996). Environmental Modelling: Fate and Transport of Pollutants in Water, Air, and Soil. A Wiley-Interscience Publication, John Wiley \& Sons, Inc. ISBN 0-471-12436-2.

Shapiro, J. (1970). A Statement on Phosphorus. Journal (Water Pollution Control Federation), Vol. 42, No. 5, Part I (May, 1970), pp. 772-775.

Shin, J.K. (1998). Eutrophication and Ecological Characteristics of Algak Population in the Freshwater Zone of the Naktong River. Ph.D. Dissertation. Department of Biology the Graduate School Inje University.

Shin, J.K., Lee, O.H. and Cho, K.J. (2003a). Algal Growth Potential Test (AGPT) for Jinyang Reservoir. Korean J. Limnol. 36 (1), pp. 57-65.

Shin, J.K. and Hwang, S.J. (2003b). Algal Growth Potential Test (AGPT) for Pyeongtaek Lake and Its Tributaries. Korean J. Limnol. 36 (2), pp. 172-180.

Shin, J. K., Jeong, S.A., Choi, I., and Hwang, S.J. (2004). Dynamics of turbid water in a Korean reservoir with selective withdrawal discharges. Korean J. Limnol. 37(4), pp. 423-430.

Shoaf, W.T. (1978). Use of algal assays to determine the potential for phytoplankton growth. Hydrological Sciences-Bulletin-des Sciences Hydrologiques, 23, 4, 12/1978. US Geological Survey, National Water Quality Laboratory-Atlanta, Doraville, Georgia 30340, USA.

Silvey, J.K., Henley, D.E. and Wyatt, J.T. (1972). Planktonic blue-green algae; growth and odorproduction studies. J. Am. Waterw. Assoc.64, pp. 35-39.

Skulberg, O.M. (1966). Algal cultures as a means to assess the fertilizing influence of pollution. Third International Conference on Water Pollution Research, Section 1:6, pp. 1-15. Washington, D.C.

Smagorinsky, J. (1963). General circulation experiments with the primitive equations: I.The basic experiment. Mon. Weather Rev. 91, 99-164.

Soballe, D.M., and Kimmel, B.L. (1987). A large-scale comparison of factors influencing phytoplankton abundance in rivers, lakes, and impoundments. Ecology 68, pp. 1943-1954.

Socolofsky, S.A. and Jirka, G.H. (2005). Mixing in Lakes and Reservoirs, In CVEN 489-501: Special Topics in Mixing and Transport Processes in the Environment. Coastal and Ocean Engineering Division, Texas A\&M University M.S. 3136, College Station, TX 77843-3136.

Solomatine, D.P. (2002). Data-driven modelling: paradigm, methods, experiences. Proc. $5^{\text {th }}$ International Conference on Hydroinformatics, Cardiff, UK, July 1-5, 2002

Solomatine, D.P. (2005). Data-driven modelling and computational intelligence methods in hydrology. Encyclopedia of Hydrological Sciences (M.G. Andersen, ed), vol. 1, John Wiley \& Sons.

Solomatine, D.P. and Dulal, K.N. (2003). Model tree as an alternative to neural network in rainfall-runoff modelling. Hydrological Sciences J. (to appear)

Solomatine, D.P. (2005). Data-driven modelling and computational intelligence methods in hydrology. 
Encyclopedia of Hydrological Sciences (M.G. Andersen, ed), vol. 1, John Wiley \& Sons.

Solomatine, D. P. and Siek, M.B. (2006). Modular learning models in forecasting natural phenomena, Neural Networks, Vol. 19, pp. 215-224

Soyupak, S. and Chen, D.G. (2004). Fuzzy logic model to estimate seasonal pseudo steady stste chlorophyll-a concentrations in reservoirs." Environmental Modeling and Assessment 9: pp. 51-59.

Spigel, R.H. and Imberger, J. (1987). Mixing processes relevant to phytoplankton dynamics in lakes. New Zealand Journal of Marine and Freshwater Research, Vol. 21, pp. 361-377.

Sridharan, N. and Lee, G.F. (1977). Algal nutrient limitation in Lake Ontario and tributary waters. Water Res. 11, pp. 849-858

Stow, C.A., Roessler, C., Borsuk, M.E., Bowen, J.D. and Reckhow, K.H. (2003). Comparison of Estuarine Water Quality Models for Total Maximum Daily Load Development in Neuse River Estuary. Journal of Water Resources Planning and Management (c) ASCE / July/August 2003 / 307 314.

Straškraba, M. and Tundisi, G. (1999) Guidelines of Lake Management Vol. 9, Reservoir Water Quality Management. International Lake Environment Committee (ILEC).

Straškrábová, V., Izmest’eva, L.R., Maksimova, E.A., Fietz, S., Nedoma, J., Borovec, J., Kobanova, G.I., Shchetinina, E.V. and Pislegina, E.V. (2005). Primary production and microbial activity in the euphotic zone of Lake Baikal (Southern Basin) during late winter. Global Planet Change 46:57-73.

Stumm, W. and Morgan, J. (1970). Aquatic chemistry, an introduction emphasizing chemical equilibria in natural water, Wiley Interscience, NY.

Straskraba, M. and Gnauck, A.H. (1985). Freshwater Ecosystems Modelling and Simulation. Developments in Environmental Modelling, 8, Elsevier, Amsterdam, 309 pp.

Straskraba, M., Tundisi, J.G. and Duncan, A. (eds), (1993). Comparative Reservoir Limnology and Water Quality Management. Kluwer Academic Publishers, Dordrecht.

Sumathi, S. and Sivanandam, S.N. (2006). Introduction to Data Mining and its Applications Series: Studies in Computational Intelligence, Vol. 29.

Sverdrup, H.U., Johnson, M.W. and Fleming, R.H. (1942). The oceans. Prentice-Hall Inc., Englewood Cliffs.

Tan, Y., Shi, L., Tong, W., Gene Hwang, G.T. and Wang, C. 2004. Multi-class tumor classification by discriminant partial least squares using microarray gene expression data and assessment of classification models. Computational Biology and Chemistry, Vol. 28, pp. 235-244.

Thomann, R.V. and Mueller, J.A. (1987). Principles of Surface Water Quality Modeling and Control. pp 91-173. Harper Collins Publishers.

Thornton, J., Steel, A. and Rast, W. (1992, 1996). Reservoirs, In Water Quality Assessments - A Guide to Use of Biota, Sediments and Water in Environmental Monitoring, Deborah Chapman (Ed.), UNESCO/WHO/UNEP.

Tilman, D. (1982). Resource competition and community structure. Princeton University Press, Princeton, New Jersey, USA.

Torréton, J.P. Bacteria. The Tuamotu atoll Communities, The Institute for Research and Development (IRD), France. http://www.com.univ-mrs.fr/IRD/atollpol/commatoll/ukbactpl.htm (5, June2009).

Uhlmann, D. (1982). Evaluation of strategies for controlling eutrophication of lakes and reservoirs. Int. Revue gen. Hydrobiol. 67, pp. 821-835.

Umlauf, L., Wang,Y. and Hutter, K. (1999). Comparing Two Topography-Following Primitive Equation Models for Lake Circulation. Journal of Computational Physics 153, pp. 638-659. 
UNEP and PHO (Panamacian Health Organization). (2005). Glossary of Environmental Health Terms. GEO Health Project

US EPA. (1971). Algal Assay Procedure: Bottle Test. National Eutrophication Research Program, Corvallis EPA1972-795-146/1, Region 10, 82p.

US EPA. (1986). Quality criteria for water. Office of Water Regulations and Standards. EPA 440/5-86001.

US EPA. (1994). Final Draft for the Drinking Water Criteria Document on Trihalomethanes. U.S. Environmental Protection Agency, Office of Science and Technology. Washington, D.C., April 8.

US EPA (2002). Guidance for Quality Assurance Project Plans for Modeling. EPA QA/G-5M. December 2002

US EPA . (2007). Carlson's Trophic State Index. Aquatic Biodiversity. http://www.epa.gov/bioindicators/ aquatic/carlson.html.

Valiela, I. (1984). Marine ecological processes. Springer-Verlag, New York, NY.

Vallentyne, J.R. (1974). The algal bowl : lakes and man. Ottawa.

Vested, H.J., Berg, P. and , Uhrenholdt, T. (1998). Dense water formation in the Northern Adriatic. Journal of Marine Systems 18, 135-160.

Versteeg, H. K. and Malalasekera, W. (1995). An introduction to computational fluid dynamics - The finite volume method. Pearson Educational Limited, Edinburgh Gate, Harlow, Esswx CM20 2JE, England and Associated Companies throughout the world. OLongman Group Ltd.

Vollenweider, R.A. and J.J. Kerekes. (1980a). Synthesis Report, Cooperative Programme on Monitoring of Inland Waters (Eutrophication Control). Report prepared on behalf of Technical Bureau, Water Management Sector Group, OECD, Paris. 290 p.

Voronov, A.G., Drozdov, N.N., Krivoluckij, D.A., Myalo, E.G. (2002). Biogeography with Fundamentals of Ecology. Moscow State University Press, Moscow, 392 pp (in Russian).

Vollenweider, R.A., and J.J. Kerekes. (1980b). Background and summary results of the OECD cooperative program on eutrophication. In International Symposium on Inland Waters and Lake Restoration. EPA 440/5-81-010. U.S. Environmental Protection Agency, Washington, DC.

Wagstaff, K., Cardie, C., Rogers, S. and Schroedl, S. (2001). Constrained k-means clustering with background knowledge. The Eighteenth International Conference on Machine Learning.

Walker, W.W. (1983). Significance of eutrophication in water supply reservoirs. Research and Technology. 0003-150X/83/010038-05\$02.00. Journal AWWA.

Walker, W.W. (1987). Empirical methods for predicting eutrophication in impoundments. Report 4, Applications manual, Tech. Rep. E-81-9. Prep. for U.S. Army Corps of Engineers Waterways Exp. Sta. Vicksburg, MS.

Walker, W.W. and Havens, K.E. (2003). Development and application of a phosphorus balance model for Lake Istokpoga, Florida. Lake Reserv. Manage. 19(1), pp. 79-91.

Wang Y. and Witten I.H. (1997). Inducing Model Trees for Continuous Classes, in Poster Papers of the 9th European Conference on Machine Learning (ECML 97), van Someren, M. \& Widmer, G. (Eds.), Prague, Czech Republic, pp. 128-137.

Wang, Y. (2003). Importance of subgrid-scale parameterization in numerical simulations of lake circulation. Advances in Water Resources 26, 277-294

Wang, Y. and Hutter, K., (2003). Numerical Modelling of Lake Dynamics. In Pandalai, S. G. (Ed.) Recent Developments in Physical Oceanography. Kerala, Transworld Research Network.

Watanabe, M.F., Harada, K.I., Carmichael, W.W. and Fujiki, H. (1996). Toxic Microcystis. CRC Press, ISBN 0-8493-7693-9. 262pp. 
Wetzel, A. (1981). Ökologische und stratigraphische Bedeutung biogener Gefüge in quartären Sedimenten am NW-afrikanischen Kontinentalrand. Meteor Forschungsergeb. Reihe C. 34, pp. 1-34.

Wetzel, R.G. (1983). Limnology. 2nd ed. Saunders College, Philadelphia, 767p.

Wetzel, R.G. (2001). Limnology: Lake and River Ecosystems. Elsevier academic press, New York.

Wheaton, F.W. (1977). Aquacultural Engineering. Wiley-InterScience, New York, New York.

Whitehead, P. and Hornberger, G., (1984). Modelling algal behaviour in the River Thames. Water Research 18, pp. 945-953.

Wikipedia. http://en.wikipedia.org/wiki/Large eddy simulation (11:40 21 July 2009). Large Eddy Simulation in Wikipedia, the free encyclopedia.

Wilson, C.B. and Walker, W.W. (1989). Development of Lake Assessment Methods Based Upon the Aquatic Ecoregion Concept. Lake and Reservoir Management, 1989 5(2): 11.22 (C) 1989 North American Lake Management Society.

Witten, I.H. and Frank, E. (2005). Data Mining: Practical Machine Learning Tools and Techniques. ISBN: 0-12-088407-0, 500 Sansome Street, Suite 400, San Francisco, CA 94111, Morgan Kaufmann Publisher.

Witzel, K.P. and Overbeck, H.J. (1979), Heterotrophic Nitrification by Arthrobacter sp. (Strain 9006) as Influenced by Different Cultural Conditions, Growth State and Acetate Metabolism. Arch. Microbiol. $122,137-143$.

Wolf, W.R. and Azevedo, J.L.F. (2005). Compressible Aerodynamic Flow Simulations Using ENO and WENO Schemes in a Finite Volume Unstructured Grid Context. TEMA Tend. Mat. Apl. Comput. 6, No. 2, pp. 325-335.

Wu, C.C. (2006). A High Order WENO Finite Difference Scheme for Incompressible Fluids and Magnetohydrodynamics. GTP Workshop on Modeling MHD Turbulence; Applications to Planetary and Stellar dynamos at NCAR, 27-30 June, 2006, Boulder, CO, USA.

Wu, F. and Tanoue, E. (2001). Geochemical characterization of organic ligands for copper (II) in different molecular size fractions in Lake Biwa, Japan. Organic Geochemistry 32, pp. 1311-1318.

Wynne, D. and Berman, T. (1980). Hot water extractable phosphorus-an indicator of nutritional status of Peridinium cinctus (Dinophyceae) from Lake Kinneret (Israel). J.Phycol. 16, pp. 40-44.

Yee, H.C. (1989). A Class of High-Resolution Explicit and Implicit Shock-Capturing Methods. NASA Ames Research Center, Moffett Field, California, 94035 USA

Zaheer and Bai, C.G. (2003). Application Of Artificial Neural Networks For Water Quality Management. Lowland Technology International Vol. 5, No.2, pp. 10-15.

Zalesak, S. T. (1989). A preliminary comparison of modern shock-capturing schemes: Linear advection. Advances in Computer Methods for Partial Differential Equations VI, R. Vichnevetsky and R. S. Stapleton, Eds., IMACS, 15-22.

Zazouli, M.A., Nasseri, S., Mahvi, A.H., Gholami, M., Mesdaghinia, A.R. and Younecian, M. (2008). Studies on Rejection and Fouling of Polyamide Reverse Osmosis Membrane in the Treatment of Water Solutions Containing Humic Acids. World Applied Sciences Journal 3 (3), 434-440, ISSN $1818-4952$.

Zhuravlev, Y.N. and Avetisov, V.A. (2006). The definition of life in the context of its origin. Biogeosciences, 3, 281-291, 2006.

Zimmerman, M.J. (1980). Aquatic Chemistry: A Chemical Equilibrium Approach. Ph.D. Thesis, The University of Georgia, Athens. 



\section{Samenvatting}

\section{Ecologisch-hydraulisch modelleren van de eutrofiëring voor het beheer van een stuwmeer}

Een stuwmeer is een kunstmatig meer dat ontstaat wanneer de vallei achter een dam, aangelegd in een rivier, onder water wordt gezet. Stuwmeren worden gebruikt voor verschillende doeleinden: drinkwatervoorziening, hoogwaterbeheersing, waterkrachtopwekking, recreatie, enz. Vaak worden stuwmeren aangelegd in gebieden waar aanzienlijk menselijke activiteit bestaat (bevolking, industrie, landbouw). Tengevolge van deze activiteiten staan stuwmeren bloot aan verontreiniging die het noodzakelijke en legitieme gebruik van water in de weg staat. Daarom zijn uitvoerige studies van de waterkwaliteit van deze stuwmeren noodzakelijk, zowel voor als na hun ingebruikname.

Ondanks bepaalde verschillen worden natuurlijke meren en stuwmeren beschouwd als systemen met vergelijkbare eigenschappen, in het bijzonder op het gebied van beheer en voorzieningen. In beide systemen heeft het water een aanzienlijke verblijfstijd. Studies van de waterkwaliteit van een stuwmeer of een natuurlijk meer omvatten doorgaans de beschrijving van drie hoofdkenmerken: de hydrodynamiek, de fysisch-chemische eigenschappen en de biologie. Traditioneel worden deze kenmerken beschreven door middel van een intensieve bemonstering van de verschillende milieuvariabelen.

Als gevolg van beperkingen van budgetaire of technische aard ontbreken in de meeste bemonsteringsprogramma's voor waterkwaliteit gedetailleerde hydrodynamische metingen die de bewegingen van waterdeeltjes omschrijven. De nadruk ligt op de algemene fysisch-chemische parameters zoals watertemperatuur, zuurstofgehalte, zuurgraad $(\mathrm{pH})$ en helderheid (zichtdiepte gemeten met een Secchi-schijf) omdat deze parameters makkelijk te meten zijn. De voorspelling van het hydrodynamisch gedrag (beweging en transport) van substanties die de waterkwaliteit beïnvloeden wordt sinds geruime tijd beschouwd als een cruciale component van elke waterkwaliteitsstudie. Stuwmeren gelegen in gebieden met een gematigd moessonklimaat ondergaan doorgaans tweemaal per jaar een sterke thermische gelaagdheid. Deze stratificatie veroorzaakt verplaatsing van opgeloste en gesuspendeerde stoffen in het meer als gevolg van verschillen in dichtheid op plaatsen met temperatuursverschillen tussen het instromende en ontvangende water. De focus van veel recente onderzoeken op het gebied van waterkwaliteit ligt daarom op twee soorten eco-hydraulische modellen: (1) deductieve modellen gebaseerd op fysische processen en gegevens over de randvoorwaarden (de zgn. physically-based models); en (2) inductieve modellen op basis van uitvoerige datasets (de zgn. data-driven models).

De verhoogde toevoer van nutriënten tengevolge van de toenemende druk op 
stuwmeren door de groeiende stedelijke bevolking en de daarmee gepaard gaande menselijke activiteit leidt to een toename van de groei van fytoplankton. Deze eutrofiëring heeft directe gevolgen voor de waterkwaliteit in het meer. Ook stoffen die gevaarlijk zijn voor de gezondheid of giftig, zoals arsenicum en zware metalen die zich hebben opgehoopt, maken een stuwmeer ongeschikt voor gebruik. Dit onderzoek concentreert zich op de eutrofiëring van een stuwmeer door belasting met nutriënten.

De belangrijkste waterkwaliteitsvariabele is fytoplankton. Naast de beschikbaarheid van nutriënten zijn er andere factoren die de groei van fytoplankton beïnvloeden, zoals licht, watertemperatuur, consumptie door fytoplankton-etende organismen, giftige stoffen, verblijfstijd, hydraulische vermenging, diverse karakteristieken van het stroomgebied zoals morfologie, grondsoort, landgebruik enz. De eutrofiëring in een stuwmeer wordt door deze factoren bepaald. Omdat een aantal van deze factoren een remmende werking op algengroei heeft moet een beoordeling van de waterkwaliteit van een stuwmeer verschillende analyses omvatten, inclusief biochemische en hydraulische vermengingsprocessen. Deze studie is gericht op de voorspelling van de waterkwaliteit door middel van biologische standaardisatie ('bioassay'), hybride modellen die verklarende modellen gebaseerd op natuurwetten combineren met beschrijvende modellen op basis van datasets, en de toepassing hiervan op een beslismodel voor de beheersing van de eutrofiëring in het Yongdam stuwmeer.

\section{Beoordeling van de waterkwaliteit}

De beoordeling van de waterkwaliteit van een stuwmeer spitst zich toe op de fysischchemische en organische parameters en de eutrofiëringsprocessen. Ondanks de korte periode van waarnemingen (2005-2007) voor het Yongdam stuwmeer onthullen de data verschillende stromingsregimes, zoals een dubbele spronglaag (een spronglaag of thermocline is de overgang tussen twee lagen met verschillende temperaturen en is optisch waarneembaar als een laagje troebel water) en turbulente dichtheidsstromingen in 2005/2006 en geen turbulentie in 2007 tengevolge van meteorologische omstandigheden zoals het neerslagregime. De weersomstandigheden hebben niet alleen een invloed op de menging van het water in het stuwmeer en de dikte van de spronglaag, die bepalend is voor het transport van nutriënten en advectie in het meer (het transport van emissies door de beweging van waterdeeltjes), maar ook op de algengroei. Desalniettemin duiden de afnemende concentraties van chlorofyl-a en fosfor tijdens de waarnemingsperiode (2005-2007) op een toenemende produktie van organisch materiaal vanuit het stroomgebied en afbraak van algen op de bodem van het stuwmeer. De toename van het chemisch zuurstofverbruik (CZV) in het gehele stuwmeer en de afname van het zuurstofgehalte in de bodemlaag gedurende deze drie jaar wijzen erop dat de eutrofiëring, sinds het Yongdam stuwmeer zich vulde in 2001, zich in de jaren 2002-2004 heeft gestabiliseerd. Dit wordt aangeduid als het verouderingsproces (rijpingsproces?). Uit de waterkwaliteitsanalyse over de volgende drie jaren (20052007) kan worden afgeleid dat het Yongdam stuwmeer een langzame limnologische ontwikkeling ondergaat in reactie op de invloeden van het stroomgebied. 
Een bioassay is de meest directe en effectieve manier om de hoeveelheden te bepalen van de verschillende nutriënten die in een watersysteem beschikbaar zijn voor organismen. Het is een soort beperkende nutriënt en eutrofiëring potentie van een aquatisch systeem. De betekenis van een bioassay voor de meting van potentiële fytoplanktongroei ("algal growth potential", AGP) in een watermonster is dat onderscheid gemaakt kan worden tussen de zichtbare concentratie fytoplankton en de potentiële extra fytoplanktongroei op basis van de beschikbare nutriënten in het monster. Een hoge AGP in het oppervlaktewater kan betekenen dat er onbekende factoren aanwezig zijn die algengroei belemmeren. De AGP van het thermisch gestratificeerde Yongdam stuwmeer laat zien dat fosfor het limiterende nutriënt is en dat in het stuwmeer verschillende zones (een rivierzone, een meerzone en een overgangszone) onderscheiden kunnen worden op basis van de vertikale en horizontale verdeling van de AGP gedurende de sterke thermische stratificatie van het water in het stuwmeer.

\section{Paradigma voor modelstudies}

In het meeste wetenschappelijk onderzoek worden waarnemingen (data) vergeleken met een theorie die gebaseerd is op een wiskundige formulering van natuurkundige wetten. Het succes van de vergelijking, of gebrek daaraan, is een test van de hypothese over de werking van de natuur uitgedrukt in wiskundige formules. Een alternatieve benadering is het schatten van een wiskundig model op basis van gemeten waarden (data), hetgeen resulteert in bepaalde natuurkundige paramaters. Physically-based models voorspellen, simuleren of genereren een verschijnsel op basis van een wiskundige formulering van natuurkundige wetten. Daarom kunnen deze verklarende modellen worden gebruikt voor verschillende doeleinden: analyse, beheer, voorspelling en planning.

Onderzoekers die gebruik maken van modellen willen de modelresultaten bestuderen binnen een samenhangend kader van ruimte- en tijdsgrenzen. Daarentegen willen beleidsmakers dat de gebruikers van de modellen de uitkomsten snel en makkelijk kunnen toepassen in het besluitvormingsproces. Dit betekent dat een theoretische generalisatie automatisch uit de modeluitkomsten afgeleid moet kunnen worden door middel van inductie. De nadelen van verklarende, driedimensionale modellen met een fijn ruimtelijk netwerk bestaan uit onzekerheid in de theorievorming en lange rekentijden. Om die reden worden één- en tweedimensionale modellen nog steeds gebruikt voor het besluitvormingsproces. De combinatie van beperkingen van hydrodynamische wiskundige modellen met een breed scala van milieuproblemen heeft geleid tot de opkomst van data-driven models als een veelbelovende nieuwe richting voor onderzoek van multivariate datasets. Dergelijke data-driven models blijken populair te zijn in veel toepassingsgebieden. In essentie bestaat data-driven modelling uit het onderzoeken van verbanden tussen een groot aantal variabelen en het verkrijgen van informatie uit de data in de vorm van formules, rekenvoorschriften, patronen en regels. Data-driven modellen hebben echter grote hoeveelheden gegevens nodig en zijn moeilijk te gebruiken voor het afleiden van proceskennis. Daarom worden ze hoofdzakelijk gebruikt voor voorspellingen op basis van tijdseries. Een hybride model, 
dat deze twee typen modellen combineert maar hun beperkingen vermijdt, vetegenwoordigt een nieuw paradigma voor een beter begrip van de werkelijkheid.

\section{"Physically-based" numerieke modellen}

Losstaande hydrodynamische studies van watersystemen, en binnenwateren in het bijzonder, zijn zeer zeldzaam. Waterkwaliteitsstudies gebaseerd op hydrodynamische modellen komen vaker voor. In dit onderzoek wordt een hydrodynamische analyse van de stroming in een stuwmeer uitgevoerd met een volledig drie-dimensionaal (3-D) hydraulisch model; en een waterkwaliteitsanalyse met een zijdelings gemiddeld tweedimensionaal (2-D) model gecalibreerd aan de hand van fysisch-chemische metingen. De meeste 3-D modellen die worden gebruikt in de fysische limnologie zijn hoofdzakelijk ontworpen voor de simulatie van de circulatie in oceanen en estuaria. Veel van deze modellen zijn succesvol toegepast op problemen in oceanen en estuaria maar er zijn weinig bekende toepassingen voor natuurlijke meren en stuwmeren. De reden hiervan is dat de fysische processen in stuwmeren anders zijn dan in oceanen. In meren wordt advectie in de momentum vergelijkingen veroorzaakt door interne golven, temperatuursverschillen, wind en instroming van de rivier, terwijl in oceanen de Corioliskrachten en drukverschillen de sturende functies zijn. Om de oceaanmodellen geschikt te maken voor de fysische verschijnselen in een stuwmeer dient een kleinere lengteschaal voor de stromingsberekeningen te worden gebruikt. Als alternatief zijn zijdelings gemiddelde 2-D modellen toegepast in stuwmeren en natuurlijke meren.

Dit is gedaan omdat deze modellen speciaal zijn ontworpen voor stuwmeren en natuurlijke meren, en sommige modelonderzoekers beweren dat het succes van deze modellen te danken is aan de nauwkeurige beschrijving van de grootschalige natuurkundige processen in meren (hoewel de modellen de kleinschalige bewegingsprocessen nauwelijks beschrijven). Deze modellen zijn veel efficiënter in het gebruik van computergeheugen en -tijd dan 3-D modellen. In dit onderzoek hebben de MIKE3D en CE-QUAL-W2 modellen, die in eerste instantie zijn ontwikkeld voor toepassing in respectievelijk oceanen en stuwmeren, goede resultaten laten zien voor de mengingsprocessen in stuwmeren. Het zijdelings gemiddelde, tweedimensionale CEQUAL-W2 model kan succesvol worden gebruikt voor het simuleren van de mengingsprocessen in het Yongdam stuwmeer, behalve wat betreft de eutrofiëring. Het driedimensionale MIKE3D model, opgezet met een 90 meter horizontaal grid en een 2 meter vertikaal grid, simuleerde de hydrodynamische processen in het stuwmeer goed. De resultaten van MIKE3D komen beter overeen met de waarnemingen van de hydrodynamische variabelen dan de resultaten van het $2 \mathrm{D}$ model, en ze laten zien dat een overstort van het meer een effect heeft op de stroming over de hele diepte, ondanks de thermische stratificatie in het meer. Dit betekent dat een efficiënte selectieve onttrekking van troebel water kleiner moet zijn dan onttrekking van water over de gehele diepte. De luchttemperatuur en de aan- en afvoer van water, en met name overstorten zijn geïdentificeerd als de belangrijkste sturende factoren voor menging in het Yongdam stuwmeer. Aangezien de parameters voor biologische processen, zoals algengroei, niet gecalibreerd konden worden met de gemeten waarden, werd de Chl-a concentratie in het stuwmeer voorspeld met een data-driven model op grond van 
gemeten data en de met CE-QUAL-W2 gegenereerde dataset.

\section{"Data-driven" modellen (DDM)}

In dit onderzoek worden drie 'machine learning' (op artificile intelligentie gebaseerde) methoden voor data-driven modellen (DDM) beproefd. Dit zijn 'Partition' (classificatie middels beslisboom of regressie), 'Instance based rule of $\mathrm{k}$ number(s)' en een 'Clustering' methode. ANN is een op artificiele intelligentie gebaseerde klassificatiemethode (een soort regressiemethode), kNN gebruikt regels voor onafhankelijke getallenparen, 'Model Trees' gebruiken een regels voor kwalificatie, en ' $\mathrm{k}$-means' is een 'Clustering' methode. Clustering identificeert getallenparen die een sterkere onderlinge overeenkomst hebben dan andere getallenparen.. De meeste numerieke methoden voor regressie-analyse gebruiken de kleinste kwadratenmethode (LSER). In dit onderzoek werd de partiële kleinste kwadratenmethode (PLSR), waarbij latente vectoren worden gevonden die zowel de predictor respons en variatie verklaren, getest als alternatief voor LSER vanwege het gebrek aan data. Model Trees (MT) staat bekend als een van de meest expliciete DDM methoden, omdat het dendritisch structuren van regressiefuncties zijn, waarin de bladeren uit meerdere lineaire regressiefuncties bestaan.

De DDM's werden getraind met metingen over twee jaar (2005-2006), waarna voorspelde concentraties van Chl-a (een output variabele) vergeleken werden met nieuwe input variabelen (water temperatuur, PO4-P en totaal fosfaat) gemeten in 2007. Volgens de uitkomsten van de toepassing van deze DDM's, bleken de combinatie van ' $\mathrm{k}$-means clustering' en PLSR betere resultaten te geven dan de andere machine learning algoritmen.

\section{Hybride modellen}

Physically-based modellen hebben ook tekortkomingen op het gebied van het voorspellen van biologische processen zoals fytoplanktonmetabolisme. Het data-driven model wordt getraind met metingen, en daarna vormen de voorspelde Chl-a concentraties (een respons variabele) met de voorspelde waarden voor variabelen (watertemperatuur, $\mathrm{PO}_{4}-\mathrm{P}$ en fosfor) uit de wiskundige modellen gebaseerd op natuurkundige wetten de nieuwe input. De resultaten zijn beter dan die van het CEQUAL-W2 model. Alhoewel het data-driven model op zich geen tijdreeksmodel is, genereert het tijdreeks gegevens zolang de input voor de voorspellingen ook uit tijdreeksen bestaat. Het hybride model complementeert het gebruik van physicallybased and data-driven modellen afzonderlijk, en geeft betere resultaten. Het kan daarom worden gebruikt voor het voorspellen van de waterkwaliteit en voor beheersdoeleinden.

\section{Ondersteuning van het bes/uitvormingsproces}

Het CE-QUAL-W2 model berekent de waterkwaliteit in elk punt van het stuwmeer op basis van het reductiescenario voor nutriënten in het stroomgebied, waarna het datadriven model de Chl-a concentraties voorspelt als een 'time-serie' met de door het CEQUAL-W2 model gegenereerde data. Ook wordt een korte-termijn voorspelling 
succesvol uitgevoerd met behulp van een exponentiële vereffeningsmethode.

Dit onderzoek presenteert een systematische benadering van de waterkwaliteitsbeoordeling, hybride modellen en beleidsondersteuning voor de beheersing van eutrofiëring in een stuwmeer. Een data-driven model dat gebruik maakt van clustering en PLSR voorspelt de Chl-a concentraties met goed gevolg. Het uitstromende oppervlaktewater van het Yongdam stuwmeer veroorzaakt een verplaatsing van verontreinigingen van de middelste laag naar de bovenste laag. Dit transport-mechanisme voor nutriënten wordt aanbevolen om de ophoping van nutriënten op de bodem van het stuwmeer te beperken. Tevens biedt dit mechanisme een mogelijkheid voor een continue algenproductie tijdens thermische stratificatie. De turbulente vermenging op micro-schaal en de invloed daarvan op de opname van nutriënten door fytoplankton, toxische componenten, weersomstandigheden etc. worden geanalyseerd voor een beter begrip van het fytoplanktonmetabolisme in het stuwmeer.

De waterkwaliteit in een stuwmeer fluctueert vaak in tijd en ruimte. Voor een beter begrip en verdere onderzoek zijn bemonstering en analyse van de waterkwaliteit in een stuwmeer de grootste uitdaging. Om de schommelingen in de waterkwaliteit in het gehele stuwmeer beter weer te geven en voldoende informatie te bieden voor het beantwoorden van de onderzoeksvragen dienen de metingen met kortere tussenpauzes en kleinere (onderlinge) afstanden te worden verricht.

Tenslotte bestaat de verwachting dat dit onderzoek een bijdrage zal leveren aan de ontwikkeling van beheersinstrumenten voor de waterkwaliteit in stuwmeren en aan het voorspellen van milieuprocessen. 


\section{About the Author}

Nahm-chung Jung was born in Kyungbook Province, Republic of Korea on 24 August, 1957. He graduated in agricultural civil engineering, Kyungbook National University in 1981. Subsequently, he served in the Korean Army, and then he started his career related to water at K-water (Korea Water Resources Corporation) in 1985. His undying interest in water developed due to the hardships caused by the Korean War, which took place in 1950 and lasted 3 years, destroying the whole nation. Great shortages of food were common. Out of hunger, children ate tree bark. His earliest memory of water was a small water bucket. He used to struggle with buckets to carry drinking water and was frustrated in starting a gasoline engine to pump up ground water for irrigation to a rice field and farm. It was very painful experience for him.

He started his career by taking part in the construction of Hapcheon Multipurpose Dam during his first 5 years at K-water. Then he moved to water works department of Kwater. He had joined the team of planning multi-regional water supply projects for several years. When he undertook the "Boryong Water Resources Development and Drinking Water Supply Project" in the central-western part of the Korean Peninsula, he had decided to study for "water of the future". In 1996, he joined the Master of Science program in Urban Water and Infrastructure Department at UNESCO-IHE. After his MSc study, he worked in the research institute of K-water from July, 1998.

His MSc study inspired him to think of water management- polluted water causes many problems in drinking water treatment processes, including disinfection by-products known to be carcinogenic. His passion for water made pushed him to start Ph.D. study to do something more meaningful even though he was in his fifties. His experience at the research institute was also another motivation for Ph.D. study in hydroinformatics at UNESCO-IHE in 2004. His Ph.D. study was promoted as a part of the research project of integrated watershed management of the Yongdam reservoir in Korea.

Thus, his $\mathrm{Ph} . \mathrm{D}$. study focused on the reservoir mixing behavior and nutrients transport mechanism, water quality (especially algal growth) forecasting and prediction due to watershed management scenarios. Most notably, he developed a data-driven modelling technique combining clustering rule, model trees and partial lest squares regression. Finally, to draw more relevant results, he proposed hybrid modelling amalgamating physically-based models, data-driven models and bioassay. This scheme of his Ph.D. study reflected his thinking and philosophy focused on the reservoir management as a decision maker at K-water. 


\title{
Stellingen
}

\author{
Behorend bij het proefschrift \\ Eco-Hydraulische Modellering van eutrofiëring voor reservoir management \\ door Nahm-chung Jung, $7^{\text {th }}$ December 2009, Delft, Nederland
}

1. "Als je iets wetenschappelijks niet in simpele termen kunt uitleggen, begrijp je het niet. Als je de betekenis van je werk niet in simpele termen kunt uitleggen, heeft het misschien geen betekenis of begrijp je zelf deze betekenis niet."

(Richard P. Feynman, 1918 1988)

Natuur is inherent complex maar wetenschappers geloven dat het in simpele termen kan worden uitgelegd met wiskunde.

2. Fysiek gebaseerde modellen maken gebruik van wiskunde om de illusie van realiteit te creëren. Milieustatistieken verklaren het verleden in termen van waarschijnlijkheid en op data gebaseerde modellen voorspellen de toekomst op grond van historische data. Deze analyses geven inzicht, maar het is niet genoeg. Het is de combinatie van deze analyses dat een nieuw paradigma brengt voor een beter begrip van natuur.

3. Resultaten van complexe modellen kunnen in simpele termen worden gepresenteerd om te helpen bij het nadenken en om de beleidvorming te vergemakkelijken voor alle wetenschappen, disciplines en perspectieven van belanghebbenden in verband met water.

4. Maatschappelijke deelname in een politiek debat leidt vaak tot degeneratie van de kwaliteit van het debat vanwege grote tegenstrijdigheid tussen de gecompliceerde technische informatie die de reservoirs en hun managers verlenen en wat begrijpelijk is voor individuele leden van de samenleving

5. Ingenieurs en/of wetenschappers zijn zeer belangrijke deelnemers in de beleidsdiscussies. Ze moeten wel duidelijk zijn over zowel tekortkomingen als optimistische conclusies, gebaseerd op hun gegevens uit het aquatische milieu, wanneer de toekomt op het spel staat.

6. Er is een oud chinees gezegde dat zegt dat een "vijftigjarige" moet "terugbetalen" aan de minder gelukkige mensen.

Daar schoon water net zo belangrijk is voor de aarde als moeders melk voor het kind, wat kan een wateringenieur beter doen dan een erfenis van schoon water achterlaten?

7. Voordat een wateringenieur spreekt, moet hij/zij niet alleen de essentie begrijpen maar ook een optimale interpretatie van het aquatisch milieu ontwikkelen voor anderen.

8. "Technische kennis is een meesterschap van complexiteit, terwijl creativiteit het meesterschap van simpliciteit is." (Zeeman, E Christopher, 1977. Catastrophe Theory).

Ingenieurs met hun technische vaardigheid zijn de makers van de beschaving en tussenpersonen tussen God en de samenleving.

9. Het schepsel van de ingenieur is een uitdaging voor zijn/haar vertrouwen in het ontwerp, want het is onderhevig aan proeven van ontelbare natuurlijke krachten en dus blootgesteld aan eventuele ramp.

10. We maken alleen een dam in de rivier, maar maken niet het reservoir achter deze. De ingenieurs die dammen maken, moeten niet van instelling zijn dat een reservoir enkel vanuit het zichtpunt van een bepaalde discipline begrepen kan worden, of enkel volgens één correct model.

Deze stellingen worden als verdedigbaar geacht en zijn als zodanig goedgekeurd door de bevorderaar Prof. Dr. Roland K. Price. 


\title{
Propositions
}

\author{
Belonging to the thesis \\ Eco-Hydraulic Modelling Of Eutrophication For Reservoir Management \\ by Nahm-chung Jung. $7^{\text {th }}$ December 2009 . Delft, The Netherlands
}

1. "If you can't explain a bit of science in simple terms, you don't understand it. If you can't explain your work's significance in simple terms, maybe it doesn't have significance or maybe you just don't understand its significance."

(Richard P. Feynman, 1918 1988)

Nature is inherently complex; but it can be explained in simple terms through mathematics.

2. Physically-based models use mathematics to create an illusion of reality. Environmental statistics explain the past in the terms of probability, and data-driven models forecast the future based on historical data. These analyses provide insights, but are insufficient on their own. It is the combination of these analyses that provides a new paradigm for a better understanding of nature.

3. Results from complex models can be presented in simple terms to aid thinking and to facilitate policy-making across all water sciences, disciplines and stakeholder perspectives.

4. Society's participation in a policy debate often leads to a degeneration of the quality of the debate due to the wide discrepancy between the complicated nature of the technical information provided by the reservoirs and their managers and what is understandable by individual members of society.

5. Engineers and/or scientists ought to be very visible participants in policy debates. They do, however, need to be clear about their shortcomings as well as their optimistic conclusions based on their data collected from the aquatic environment when the future of the real world is at stake.

6. An old Chinese saying states that at the age of "fifty" one should "pay back" to people who are less fortunate than you.

Because clean water is as important to the earth as a mother's milk to the infant, what better is there for a water engineer to do than to leave a legacy of clean water.

7. In order for a water engineer to speak, he or she has not only to understand the essence but also develop an optimal interpretation of the aquatic environment for others.

8. "Technical skill is mastery of complexity while creativity is mastery of simplicity." (Zeeman, E Christopher, 1977. Catastrophe Theory).

Engineers with their technical skill are creators of civilization and intermediaries between God and society.

9. The engineers' structure is a challenge to their faith in the design because it is subject to trial by the myriad of natural forces in play and is therefore exposed to possible failure leading to disaster.

10. We create a dam across a river, but we do not create the reservoir behind it. Engineers who create dams should not have the attitude that a reservoir can be understood from the point of view of one particular discipline or from just one right model.

The propositions are considered defendable and as such have been approved by the promoter Prof. Dr. Roland K. Price. 
